
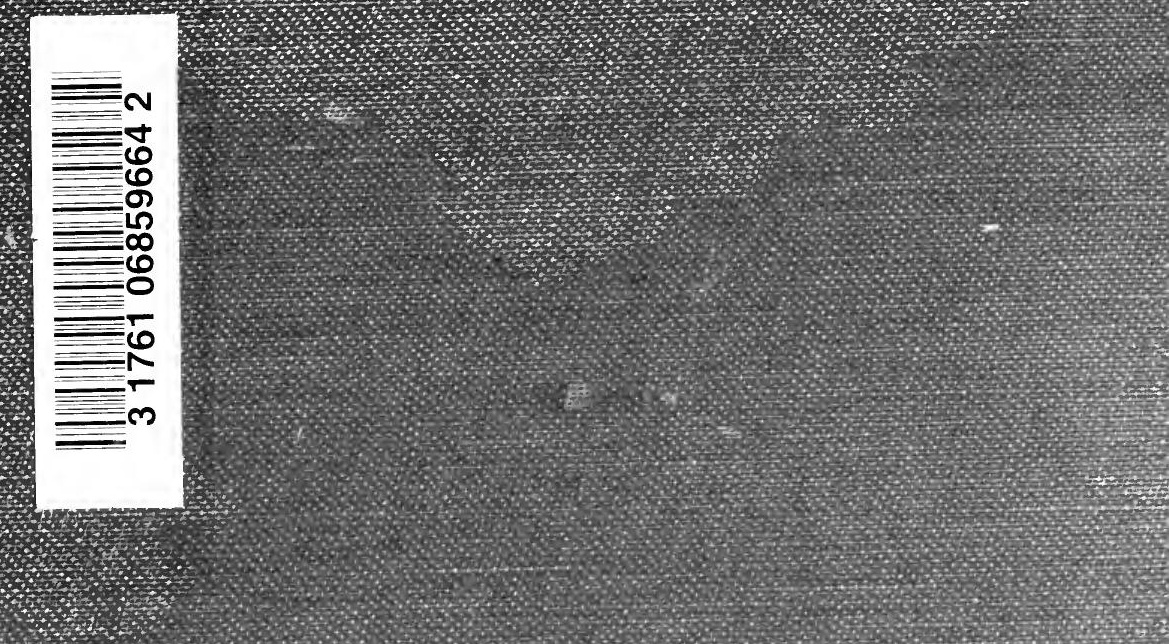





\section{SPIRITUAL PLURALISM}

AND

RECENT PHILOSOPHY 


\section{CAMBRIDGE UNIVERSITY PRESS}

C. F. CLAY, MANAGER

LONDON : FETTER LANE, E.C. 4 NEW YORK : G. P. PUTNAM'S SONS BOMBAY

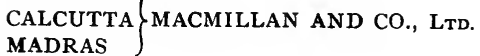
TORONTO : J. M. DENT AND SONS, LTD. TOKYO : MARUZEN-KABUSHIKI-KAISHA

ALL RIGHTS RESERVED 


\section{Philos}

Rं521

\section{SPIRITUAL PLURALISM AND}

RECENT PHILOSOPHY

BY

C. A. RICHARDSON, M.A. (Cantab.)

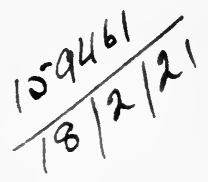

CAMBRIDGE

AT THE UNIVERSITY PRESS

I 9 I 9 



\section{TO}

JAMES WARD

FROM THE INSPIRATION OF WHOSE TEACHING THIS BOOK DERIVES ITS MAIN THEME 



\section{PREFACE}

LTHOUGH my main purpose in this volume has $A$ been to present and defend the theory that the texture of the Universe is through-and-through spiritual, it will be evident to the reader that the particular form of presentation has been considerably influenced by the teachings of a school of thought which appears at first sight consistently to oppose any kind of idealistic or spiritualistic interpretation of the world. I refer to that school which is represented in America by the neorealists, and in this country by logical atomists of the type of $\mathrm{Mr}$ Bertrand Russell. Differing widely from them on certain fundamental points, I cannot but agree with a portion of the results of their critique. Nevertheless I am convinced that in the main (except on some questions of epistemology) these results are not only not incompatible with that metaphysic which acknowledges the reality of the subject of experience and takes spiritual pluralism as its starting-point, but are actually complementary to it.

On the other hand, I am no less convinced that discussion will continue at cross-purposes, and consequently no satisfactory progress will be made, until the neo-realists or their English allies proclaim in a precise and unambiguous fashion what they are going to do about the subject of experience. I am aware that their present tendency is rather to give the impression that they think that the latter may be ignored or dispensed with altogether. But I am by no means satisfied that

R. S. P. 
they really do ignore or dispense with it in their own arguments. There is a suggestion of camouflage about the whole affair. For not only do they state that there are such things as sense-particulars, images, etc., but they seem plainly to admit that there is also such a thing as the experiencing of these objects, the experiencing of an object being by no means identical with the object itself. Very well, but will they please tell us a little more plainly exactly what they believe about this "experiencing" as distinct from the object experienced, and whether they think that no such individual as a subject is involved in it, and if not, why not? For after all, the theory which takes the existence of the subject for granted gives a perfectly clear and concise account of what it means by the experiencing of an object, and it cannot be said that neo-realism has as yet provided a satisfactory equivalent for this account. It is true that Mr Russell, in the last of a series of lectures recently delivered in London ${ }^{1}$, states that there is an empirically given relation between two experiences which constitutes their being what is commonly called "experiences of the same person," and says that we might therefore regard the person simply as the particular series of experiences between which this relation holds, dispensing with him altogether as a metaphysical entity. But think of all that may perhaps be hidden beneath such an apparently innocent phrase as "empirically given." And there is still the distinction between the series of objects and the series of experiences to be made clear.

1 These lectures have now been published in the Monist, and the particular reference here is to pp. $373 \mathrm{ff}$. of the July I 9 I 9 number of that journal. 
I believe that a definite statement from the neo-realists on this point, is one of the most pressing needs of current philosophy.

As regards the contents of this book, the greater portion now appears for the first time. The first essay, however, and the substance of the fourth, were published in the numbers of The Philosophical Review for May I 918 and Jan. I919 respectively, while the third essay was published in Mind of Jan. 1919. I should like to express my gratitude to the editors of those journals for permission to include in this volume the essays referred to. I have made slight alterations in the latter to avoid needless repetition, though in certain cases, where a point has appeared to me particularly important, I have allowed the repetition to remain, regarding it as justifiable in the circumstances.

To my former teacher, Dr James Ward, I owe a debt of gratitude the measure of which I cannot adequately express. From both his written and spoken word I have received invaluable help, while throughout my work his advice and encouragement have continually sustained me.

Among others too numerous to mention individually, I am greatly indebted to the works of $\mathrm{Mr}$ Bertrand Russell; while for the materials on which are based the preliminary descriptions of the various types of abnormal phenomena discussed in the eighth essay, I have largely to thank Sir W. F. Barrett's two books Psychical Research and On the Threshold of the Unseen. My grateful thanks are also due to Prof. James Seth (who, indeed, first suggested to me that I should publish in a book the results I had so far reached) for 
valuable comments and advice; and to my wife for the very considerable labour she has expended in the preparation of the manuscript for the press.

In conclusion, I should like, if I may, to thank the Syndics of the Cambridge University Press for their generosity in helping me on my first philosophic venture by the publication of this volume. I am only too well aware of its many shortcomings. But my excuse must be in part that it is only meant to be preliminary and tentative. I hope later to put forward a fuller exposition of my beliefs, in the development of which I shall be greatly aided by whatever reception may be in store for the present book. Hence I shall be extremely grateful for any criticism, however adverse, that the latter may call forth.

C. A. R.

22 August 1919. 


\section{CONTENTS}

\section{INTRODUCTION .}

I. SCIENTIFIC METHOD IN PHILOSOPHY AND THE FOUNDATIONS OF PLURALISM

I. Introductory. II. Outline of Scientific Method. III. Outline of Pluralism and the Genetic Method. IV. Points of Conflict between Pluralism and the "New Realism." V. The Existence of the Self. VI. Continuity. VII. Causality. VIII. Other Categories of Experience. IX. Summary and Conclusion.

II. ON CERTAIN CRITICISMS OF PLURALISM

I. Introduction. II. Externality. III. Consciousness. IV. The Evolution of Law. V. The "Bare" Monad. VI. Summary and Conclusion.

III. THE PHILOSOPHICAL PROBLEM RAISED BY THE WEBER-FECHNER LAW .

I. Introduction. II. Examination of Conclusion drawn from the Weber-Fechner Law. III. The Nature of Sense-Experience. IV. Summary and Conclusion.

IV. THE NOTION OF A DETERMINISTIC SYSTEM .

I. Introduction. II. The Case for Determinism. III. Analysis of Determinism. IV. Determinism and the Mind. V. The Problem of Free-Will. VI. Summary and Conclusion.

V. THE INTENSITY OF SENSE-DATA • . I55-I6I

VI. IMMORTALITY . . . . . . . 162-I80

I. Introduction. II. The Logical Statement of the Problem. III. Concrete Significance of the Problem for the Individual. IV. The Unity of Experience. V. Summary and Conclusion.
PAGE

xiii

I -64

$65-83$ 
VII. THE RELATION OF MIND AND BODY

I. Introduction. II. True Meaning and Implications of the Problem. III. The Pluralistic Interpretation of the Matter. IV. Application of Pluralism to the Solution of the Problem. V. Memory and Imagination and their Dependence on the Body. VI. Personality. VII. Summary and Conclusion.

VIII. SUBCONSCIOUSNESS AND CERTAIN ABNORMAL PHENOMENA . . . . . 244-328

I. Introduction. II. Ultraliminal Impressions. III. Subliminal Images. IV. The (so-called) Subliminal Self. V. Abnormal Phenomena. VI. Conclusion.

CONCLUSION .

INDEX . . . . . . . . . $333-335$ 


\section{INTRODUCTION}

THEN a man sets out to write a book on philosophy, he is faced with a difficulty at the very outset. Nor is this difficulty one to be lightly overcome, for it consists in a doubt as to what the book ought to be about if it is to merit the title of "philosophical" at all. Many, and often widely diverging, are the definitions that have been given of the philosopher's task. While on the one hand it is asserted that philosophy is the enquiry into the ultimate nature of reality ${ }^{1}$ (whatever an ultimate nature may be as distinct from any other kind of nature), on the other it is maintained that the only possible knowledge is that set forth in the results of the so-called "positive" sciences, to the unifying and co-ordination of which the philosopher must accordingly be content to confine his attention. Yet another view is that, even with regard to the body of knowledge constituting positive science, philosophy should merely investigate the form thereof without reference to any particular content ${ }^{2}$. Opposed to this vision of an arid desert of abstractions, we have the more human and idealistic tendencies to deal with life and experience in its concrete fullness and variety; to enquire into the goodness or evil, the beauty or ugliness of our own familiar world; to determine the meaning of Man's struggles, and the goal of his endeavour.

${ }^{1}$ Cf. Dr McTaggart's definition of philosophy.

2 This view is apparently held by Mr Russell. 
Probably the persistency with which every one of the above views has ever and anon been urged, is accounted for by the fact that each contains a kernel of truth, for each envisages the high aim of philosophy from one particular point of view. The special task to which one school after another would urge all efforts to be bent, represents in every case a philosophic end secondary to the ultimate end of all such thought. General agreement as to the nature of the latter is, as we have seen, unlikely to be achieved; but it is probable that most people would agree that the philosopher's search might be deemed to have ended satisfactorily if he should succeed in formulating an hypothesis which could truly be said to explain and interpret the world as we know it, and not merely to describe in the simplest possible way the relations and sequences of phenomena.

Now the origin of knowledge is experience. Juggle it as he may, cloaking it under a hundred different disguises, it is with experience-and his own experience at that-that the philosopher starts, and must start. The results of his analysis of experience, experience considered with reference to its subjective and its objective factors, its phenomenal aspect and its noumenal implication, and the point of view to which his results lead, will run, like a dominant theme in a symphony, throughout the structure which he subsequently erects.

The stages in the philosopher's pursuit will then be threefold. The first consists in the consideration of experience, and closes with the arrival at an ontological hypothesis to which that consideration leads. A necessary condition which this hypothesis must satisfy, is 
that it shall interpret and explain as opposed to merely describing. The next stage is the development of the hypothesis, a consideration of its claims as against rival hypotheses, and an investigation of its general bearings. In the third and final stage, the hypothesis is tested by applying it to the solution of particular philosophic problems. Complete demonstration or verification can never be attained, but a continually increasing degree of probability may be progressively exhibited, as the applications become at the same time more numerous yet more co-ordinated.

The following series of essays marks a consistent attempt to advance a few steps along a road such as that indicated in the foregoing. In them a particular view of experience-idealistic in its general features - is adopted, and an endeavour made to establish a certain ontological hypothesis-spiritual pluralism. Finality has not been aimed at. Indeed, it is expressly recognized that while this hypothesis is perhaps the only one from which we can make a satisfactory advance, and while it will take us a long way towards the solution of the difficulties which beset us, it yet needs to be supplemented (though by no means supplanted), by a further postulate. The writer's aim has thus been largely tentative. He will be amply satisfied if he has succeeded only in expressing his convictions lucidly, in clearly exhibiting the results to which he has so far been led, and in indicating the path along which, as it appears to him, further progress must be sought.

The essays, though each is more or less complete in itself, form the development of a single coherent line of thought. In the first essay, the essential features 
of the view of experience to be adopted are explained, and the general bearing of this view on questions of space, time, and the more important categories, is indicated. In the course of this exposition, the hypothesis of spiritual pluralism is reached inductively, and some of its most general applications are considered. To bring out the points involved more clearly, and incidentally to give additional support to the position maintained, there is throughout a reference, by contrast and comparison, to current schools of thought, particularly to that associated with the names of $\mathrm{Mr}$ Bertrand Russell and the New Realists, whose position differs in some of its most fundamental features from that taken up by the writer.

The second and third essays are attempts to strengthen the general thesis. The former deals with the most important direct criticisms of spiritual pluralism which have beenadvanced in recent philosophic writings. A distinction is made between that portion of the criticism which is aimed at the fundamentals of pluralism on the one hand, and that which, on the other, points out the necessity under which the pluralist ultimately finds himself of supplementing his hypothesis by a further principle. It has been found necessary to analyze carefully the exact meaning of the criticisms considered, and to disentangle, in at least one respect, two factors which appeared as a result, but one of which bears directly upon pluralism, the other having rather a general bearing upon all philosophic hypotheses.

In the third essay, the view of experience adopted throughout (which, as mentioned above, is idealistic in most of its essential features) is maintained as against 
its chief contemporary rival, the view held by NeoRealism. The avenue of approach to the discussion is somewhat unusual, namely through a well-known psychological law. It becomes apparent in the upshot, that the latter has extremely important philosophical bearings. The chief issue between Realism and Idealism is found to depend on the consequences we deduce from it. Having led up to the issue in this way, the discussion is continued on other grounds. In the course of an investigation of the nature of experience, it is pointed out that the analysis leads to results which necessitate a view idealistic in its essentials as opposed to realistic, while in addition certain inherent contradictions developed in views of the latter type are exposed. The essay closes with a brief discussion of pluralism as the hypothesis to which the conclusions reached in regard to experience point.

The position to be taken up having been explained in this way, and the reasons in support of it defended, it becomes necessary to test the results so far obtained by applying them to the solution of particular problems. For this purpose, the classical problems of Free-will, Immortality, and the Relation of Body and Mind, have been selected for discussion. The fourth essay deals with the first of these. The question is approached by a statement and an analysis of determinism, for the latter is commonly accepted as the contradictory of the libertarian position. It appears later, however, that each view is really significant only of one particular type of entity, and the types concerned in the two cases are quite different. An elucidation of this point suffices to prove that the subject of experience is not a type of entity to 
whom determinism is applicable, so that the universe, whatever else it may be, is not a deterministic system. So far as the major part of the discussion is concerned, the conclusions are demonstrated by the investigation of the experience of any single individual, without reference to a particular ontological hypothesis. The latter becomes necessary when it is required to extend the results obtained to the case of the universe as a whole. The fifth essay is merely an addendum to the fourth, being the detailed discussion of a psychological point raised in the latter. Although a settlement of the question involved is unnecessary so far as the solution of the free-will problem is concerned, it is yet of considerable general importance as regards both psychology and certain philosophical issues. It has therefore seemed worth while to consider it in some detail.

In the sixth essay, the most general aspects of the problem of immortality are examined. These are so often entangled with particular questions such as those relating to the nature of a future life (if any) beyond this body, which have nothing whatever to do with the general problem of immortality, that it is necessary carefully to distinguish the issues involved. The investigation hinges largely upon the position adopted with regard to the nature of time. The latter, already briefly discussed in the first essay, is now examined more fully. Just as is the case with the problem of free-will, the question as to the immortality of the individual is found to admit of an answer, at any rate as regards its wider aspects, capable of being deduced from the consideration of the experience of the individual alone, without calling in the help of a metaphysical hypothesis 
of the structure of the universe as a whole. Recourse to such an hypothesis only becomes necessary if we wish to estimate the consequences to other individuals of the facts arrived at in connection with any particular individual. It appears finally that the results in regard to the latter are summed up implicitly in the position adopted throughout with reference to one aspect of experience, its unity. The essay accordingly concludes with a development of this aspect.

The relation between mind and body is dealt with in the next essay. To no other problem does the atmosphere of the out-of-date Cartesian dualism cling so closely and persistently. It is apparent in the treatment of the issue by nearly every school of thought. Consequently before any attempt can be made at a solution, it is found necessary to clear the problem of all its ancient dualistic cobwebs, and to re-view it from the standpoint of individual experience. In the light of this, such terms as "mind," "matter," "body," are re-interpreted, and the problem is then capable of being stated in more exact terms. To attempt its solution, however, it is found requisite to advance from the consideration of a single individual experience to an investigation of the relations subsisting between individual subjects. It thus becomes necessary to make use of the hypothesis of spiritual pluralism, and by its application a solution of the problem is advanced, so far as it appears to be soluble; that is, the matter is pushed until we are left with indefinables whose nature is actually realized, though it defies descriptive statement. The conclusions reached are employed in the further discussion of a question of great theoretical and practical interest, 
namely the nature of memory and imagination, and their dependence on the body. This involves a statement of the noumenal implications of experience, in the light of our hypothesis. Finally, we are led on to the question of personality, and hence by a natural transition to a brief review of the possibilities of a future life beyond this body, a topic which was not touched upon in the previous essay.

At various points in the thesis developed in the essays so far briefly outlined, it necessarily happens that the chief types of ordinary phenomena are analyzed and interpreted. But there remains a large class of phenomena which calls for special consideration, not only because definite knowledge about it is continually increasing and pressing for co-ordination, but also because it has up till now been found impossible to bring such phenomena into satisfactory connection with the principles of existing sciences as at present stated. The phenomena are those commonly described by that term of not very happy associations-_spiritualistic." In spite of the sneers of certain bigoted scientists and others of narrow outlook, the labours of psychical researchers have established abundant and indisputable evidence of the main facts, and their results show that we have here a matter of high importance calling for very careful consideration. In particular, it is necessary to bring this new body of knowledge into relation with that already existing. For these reasons, the interpretation of phenomena of the kind mentioned, has been chosen as the task to be attempted in the final essay. We are here brought most closely face to face with the necessity of supplementing spiritual pluralism by some 
principle of unity. Such a principle is therefore postulated, although the determination of its exact nature is unnecessary for the matter in hand. With our hypothesis thus supplemented, it is found possible to coordinate the various types of abnormal "spiritualistic" phenomena, and to offer an explanation of them which may perhaps be claimed to be as satisfactory a one as can be obtained with the data at present available. It seems reasonably certain that these data, so far from meriting nothing but ridicule on account of the frauds frequently practised in connection with them, should give, if properly handled, a deeper insight into the real nature of the universe than that provided by any other group of facts.

With the position thus established, strengthened, and tested, the writer has halted for the moment. But it is evident in what direction the next advance must be attempted. When pluralism has done its utmost, we are left to search for the nature of that concrete universal principle or entity, whereby subject is linked to subject, and the many made one. 



\section{SCIENTIFIC METHOD IN PHILOSOPHY AND THE FOUNDATIONS OF PLURALISM}

\section{INTRODUCTORY}

THE opening years of the twentieth century have witnessed, among other things, the gradual emergence of a new school of philosophic thought, and a new philosophic method. To the former, which originated in America, the name of 'New Realist' has been given. The exponents ${ }^{1}$ of the latter designate it the 'Scientific Method.' New Realism is largely imbued with the spirit of the scientific method, and, for the purposes of criticism, the two may be treated together. The new method claims ${ }^{2}$ to make an entirely fresh start in philosophy, to discover what problems are capable of solution, and to open the road to that solution to those who are willing and able to make the requisite effort.

The reason for this new departure in philosophy is not far to seek. The last half of the nineteenth century gave birth to changes in the world of science of a revolutionary nature. To take two examples alone, Darwin's

${ }^{1}$ Whitehead and Russell.

${ }^{2}$ B. Russell, Our Knoweledge of the External World, Lect. I and conclusion of Lect. VIII. Mr Russell designates his particular type of realism, "logical atomism."

R. S. P. 
doctrine of Natural Selection and Maxwell's Electromagnetic Theory brought about an entire change of outlook in their respective domains of biology and physics, with a consequent overhauling of all the traditional concepts and principles. It is a commonplace that science and philosophy invariably act and react on one another. The scientific revolution was followed by an upheaval in philosophy. Everything went into the melting pot. There emerged a confused mass of opinion following upon the attempt to fit new facts to old systems, which finally settled down into two main tendencies of thought, the one clinging as far as possible to a modified Absolutism based on Kant and Hegel, the other launching out more boldly in an attempt to apply Evolution as a comprehensive metaphysical formula. The latter, though now generally discredited even by its posterity, was the forerunner of the modern evolutionary and pluralistic schools.

While much of the old remained in the new, there was, of necessity, a vagueness both in ideas and in method. Philosophy was cumbered with a mass of useless metaphysical cobwebs. Inevitably there arose an increasing demand for a general clearing up with a view to a fresh start. In this demand the new ${ }^{1}$ scientific method finds its motive force, and history is repeating the story of Descartes over again.

Schools of philosophy may be classified according as they differ in method or in system. Whichever mode of classification is adopted, the various schools fall, broadly speaking, under two main headings. In method

1 'New,' that is, in its particular mode of application of scientific principles to philosophy. 
they are Empirical or Rationalist; in system, Pluralist or Singularist. Empirical method and pluralistic belief tend to go together, for if we appeal for the most part to the crude data of sense, we are confronted by a manifest plurality. On the other hand, the craving for unity has constantly caused men to mistrust the world of sense with its eternal diversity and flux, and led them to seek the characteristics of the totality of existence by pure thought alone. Hence the method which leads to the singularist or absolutist view of reality is essentially rationalistic.

We can best trace the path of progress if we observe the development and interaction of the two classical methods of attacking the problem. Most great advances in philosophy have consisted in a partial synthesis of Rationalism and Empiricism. Kant's work is a supreme example of such a synthesis. The type of advance is analogous to that of the Hegelian dialectic. In emerging into antithesis after each successive synthesis, Rationalism ever tends to include more empirical data in the material with which it works, while Empiricism is inclined to stray ever further from the surface of things by building up its data into abstract intellectual constructions, and by framing more or less abstract hypotheses to account for these data.

Pluralism, with its genetic method, is the modern outcome of Empiricism. The position of Rationalism is not, at first sight, so clear. One's thoughts turn naturally to the idealism of the Absolutist school; but although the beliefs of this school are upheld by some of the foremost philosophers of the day, they represent an influence which is rapidly on the wane. The true pro- 
gressive product of Rationalism, despite the fact that its data are mainly empirical, is the New Realism, for its scientific method purports to deal with the form and structure of existence as opposed to its concrete content.

The final synthesis of the two points of view consists not in an amalgamation, but in a recognition of the fact that each is necessary to the complete fulfilment of philosophic purpose, and in a determination of the particular function, domain, and limitations, of each associated method.

\section{Outline of Scientific Method}

The scientific method ${ }^{1}$ attacks the problem of the Universe piecemeal. A problem is selected, isolated as far as possible, and an attempt made to clarify our conceptions relating to it, and to determine, by continued analysis, the true source of the perplexities underlying the question. The final step, and the most difficult, is to formulate an hypothesis which will resolve these perplexities. The difficulty of this last process lies in the fact that the necessary hypothesis is inevitably of a peculiarly abstract nature, for at each successive stage of the analysis the matter under consideration becomes more abstract.

In any particular investigation, the initial data consist in the generally accepted body of knowledge on the subject. This knowledge will almost invariably be vague and confused, and the task of analysis is to resolve

' B. Russell, op. cit., beginning of Lect. VIII. 


\section{Outline of Scientific Method}

it into a number of definite propositions. The latter, when the ultimate premises have been reached, are arranged in logical sequence. The premises should be stated with the minimum of redundance. They must then be scrutinized in turn with a view to ascertaining the particular degree of doubt or certainty attaching to each. This provides us with a criterion of the doubtfulness or certainty pertaining to each proposition of the sequence, and to the initial data in particular.

In general the scientific method makes use of three types of data ${ }^{1}$ : sense-data, the testimony of others, and certain primitive logical truths. In making use of testimony, the existence of other people must be tacitly assumed. It is impossible entirely to justify this assumption. On the other hand, its falsity cannot be established, and it is indispensable in opening up a relatively immense field of knowledge, whereas solipsism is practically barren.

One of the most important applications of the scientific method is to the analysis of the meaning of the concepts of physical science ${ }^{2}$, and the investigation of their validity as representative of the world of fact. Evidently, the material from which the start must in this case be made, is the data of sense. Since all scientific observation consists in perceiving sequences of sensedata, and since the verification of a physical prediction lies in an appeal to the occurrence of some sense-datum, it follows that if the entities of the physicist are to be valid conceptions, they must be capable of representation as logical functions of sense-data. Even if they be

${ }^{1}$ B. Russell, op. cit., Lect. III.

- $\quad{ }^{2}$ Ibid., Lect. IV. 
so represented, it does not follow necessarily that they exist concretely, nor does it matter. The importance of such a critique of physical science is great, for the physicist is often apt to consider his entities as the true realities of the universe, regarding them as inferences whereas they are merely constructions. The data of sense are the indubitable concrete facts.

The exponents of the scientific method claim that it is capable of ascertaining all that is soluble in the problems of philosophy, and of effecting the solution. They regard it as giving the method of research in philosophy just as mathematics gives the method of research in physics. It alone is capable of yielding whatever objective scientific knowledge it is possible to attain ${ }^{1}$. This is certainly true enough so long as we regard merely the objective side of experience as such. For the task of science is twofold. It has first to record and correlate the particular concrete facts of experience. This is the function of the specialized sciences. But the forms of the particular facts and the general aspects of experience have also to be investigated. This investigation falls to the lot of the philosopher. The things he deals with in performing it are therefore essentially abstract.

The function of the scientific method, then, though in part constructive, is mainly critical. Its field is the objective side of experience, and its scope is comprised in the determination of the validity of the concepts we apply to this objectivity, and in the solution of certain problems by the construction of other valid concepts and hypotheses. This construction it performs by analyzing

$$
1 \text { Op. cit., Lect. VIII, conclusion. }
$$


the general forms of experience. All questions such as, for example, ethical problems, are therefore regarded as outside the scope of philosophy, for they are considered to deal with the particular characteristics of the particular things composing the world ${ }^{1}$.

Although the results obtained by the scientific method may be mathematically accurate, and therefore a complete solution of the type of problem with which it deals, it will be seen later that the data from which it starts introduce an element of inadequacy. Moreover, the question arises as to whether the problems considered are the only ones with which philosophy may legitimately deal. It has commonly been considered that one of the supreme tasks of philosophy is to provide an explanation of the facts of experience. The hypotheses yielded by the scientific method are evidently purely descriptive in type. Can any hypothesis, however, be considered explanatory, or are all hypotheses descriptive, differing merely in the entities in terms of which the description is expressed? It will shortly be seen that one type of hypotheses may be considered as truly explanatory in an entirely unique sense. In this connection it is of the utmost importance to remember that there is both an objective and a subjective side to every question. Just as much light will be thrown on a problem by an investigation of the manner in which we have arrived at our relevant concepts on the subject at our present level of experience, as by an investigation of what the precise meaning of the concepts must be, if they are to

1 All New Realists do not take up this attitude with regard to ethical and analogous problems-e.g., R. B. Perry in Present Philosophical Tendencies, Ch. xiv. 
be validly representative of objective experience. The concepts we have formed may involve unwarrantable assumptions if applied uncritically to objective experience as such, but if their true meaning for us, and the manner in which that meaning has been acquired, are analyzed, not only shall we realize our own nature more clearly, but there is a possibility of the suggestion of fruitful hypotheses as to the nature of existence generally. We are thus led naturally to the consideration of pluralism and the genetic method.

\section{Outline of Plukalism and the Genetic Method}

Pluralism is based on the existence of the self. All philosophers do not believe in the existence even of one self. Reasons will be stated hereafter, however, which seem to render doubt on the subject not only logically impossible, but inherently meaningless. The word 'self' will be used as synonymous with 'subject of experience.' All confusion with the various meanings of the empirical self, which relate essentially to the self as conceived, will thus be avoided. For the class of selves or subjects considered in relation to their experience, the term ' mind' will be used. This meaning appears to approximate most closely to the general usage of that somewhat vague term.

Starting from matter, i.e., from matter as generally conceived by physics and by the main body of common sense, it is impossible to bridge the apparent gulfs between the inorganic and the organic, and between Life 


\section{Outline of Pluralism and Genetic Method 9}

and Mind. Herbert Spencer's work bears eternal witness to this fact. The only alternative, therefore, is to start from Mind and endeavour to work back. This is the endeavour of pluralism. The pluralistic hypothesis is briefly as follows: "Reality comprises selves (i.e., active subjects of experience) alone, differing simply in degree or in kind of mental development, though the diversity is indefinitely various." Experience, then, consists in action and reaction between self and other selves, described by Professor James Ward in the expressive phrase "mutuum commercium." The meaning of 'acti'vity' is considered to be fundamentally realized by everybody, but to this point we shall return.

The comparative hopes of a solution of the problems of philosophy held out respectively by Pluralism and by any form of Materialism, are sufficiently indicated by comparing the start made from the existence of selves as a basis, with that based on the existence of ultimate material particles-atoms, corpuscles, electrons, or whatever they may be considered to be. We know that some selves exist ${ }^{1}$. Strictly, each of us knows that one self exists; but, as we have seen, if we are to philosophize to any extent worthy of the name, we must take a further step and assume the existence of other selves, nor can this assumption in any way be demonstrated to be false. It is therefore justifiable to make it. On the other hand, even should the material particles of physics actually exist (and this seems very doubtful), we could not know of their existence. The scientific method demonstrates this sufficiently. Moreover, if, as that method shows to be extremely probable, the entities of physics are simply

${ }^{1}$ See Section V below. 
constructions of sense-data, we cannot conceive a self in terms of these entities; for evidently a self cannot simply be a logical function of sense-data. To sensedata we apply the term 'phenomenal,' i.e., 'presented to a subject,' thereby implying that we realize the fundamental distinction in kind between the subject and the sense-data or phenomena which it perceives. It is impossible, therefore, to imagine that we ourselves can be analyzed into sense-data; in fact, the supposition involves a contradiction in terms, for sense-data are 'given' or 'presented' by the very meaning of the term, and it is the self to whom they are presented.

Admitting, then, the existence of at least some selves, is it possible to explain the facts of experience entirely in terms of selves? As a matter of fact, the explanation has already been partially accomplished, and modern pluralists are engaged in applying it to the remaining difficulties. While at the present stage there seems to be no reason for supposing their attempts will be unsuccessful, many pluralists are of opinion that the bare hypothesis as stated above is incomplete. It seems probable that, for the complete solution of the problem, pluralism must be supplemented by some form of Theism ${ }^{1}$. However that may be, the pluralistic hypothesis is admissible until disproved by fact, and therefore it is justifiable to continue to apply it as far as possible.

It is no part of our present purpose to analyze in detail the application of pluralism to the solution of philosophic problems, but the type of method adopted may be briefly illustrated by a consideration of its ' E.g., J. Ward, Pluralism and Theism. 


\section{Outline of Pluralism and Genetic Method I I}

application to the case of what is commonly called 'inorganic matter.' The chief feature of that class of sense-data from which we construct the concept of inorganic matter, is the uniformity of the sequences manifested therein. There seems to be no expression of individuality observable. The opposite is true of selves. A self is essentially individual, for it is unique. In fact it is only to a self and its particular experience that we can correctly apply the term 'individual.' Animals manifest individuality and we have every reason of analogy to regard them as selves or subjects of experience. The animal merges insensibly into the vegetable world, and there is little difficulty in applying the pluralistic hypothesis to the latter. Now one fundamental characteristic of mind is its plastic retentiveness, which is manifested in the formation of habits. The lower we go in the scale of life, the more habit do we find, and the less spontaneity. The latter, however, and consequently individuality, never entirely disappear. Remembering how narrow is the line dividing the organic from the inorganic, we are led to regard the latter as constituted by individual agents of extremely inferior mentality, whose behaviour is therefore sufficiently habitual to admit, for the most part, of description in general terms. The reason for the apparent absence of individuality is that we are here probably dealing with individuals in bulk, so that our results are statistical. These results will be even more uniform than the majority of statistics, on account of the nature of the individuals concerned; but there is no reason to suppose that, if we could observe the behaviour of one of these individuals in isolation, we should be unable to observe 
any traces of uniqueness. The above is but a broad outline of the pluralist argument as applied to the inorganic world, and if the question is examined in greater detail, many other reasons can be adduced which show that there is nothing incompatible with experience in this view of apparently lifeless matter.

The first stage in the growth of a pluralistic philosophy is analytic. It consists in the analysis of experience, perceptual and conceptual, and of those particular concepts which we apply to experience under the name of categories. The investigation leads in all cases to results which suggest a pluralistic hypothesis, although they do not lead to it as a strict logical necessity. The second stage in the process consists in the application of the hypothesis to the solution of the particular problems of existence. In the first stage the investigation takes the form, for the most part, of an analysis of the growth of individual experience, and of the transition by intersubjective intercourse to universal conceptual experience. Hence the method employed is genetic. In this way we determine the process by which we have arrived at such knowledge as that of space and time, for example, and at such conceptions as Causality, Quality, and Relation. Thus abundant light cannot fail to be shed on the time-honoured problems associated with these names.

Pluralism is an hypothesis, and it therefore stands under the universal limitations inherent in the nature of hypothesis in general. For a just appreciation of values, then, it is necessary that these limitations should be precisely stated and clearly borne in mind.

We usually look upon hypotheses as put forward to 
'explain' certain groups of facts. Let the case of physical science serve as an illustration. As a rule, men of science are content to dismiss the data of sense as merely 'subjective.' They look upon them as due to the action of certain hypothetical entities on our senses. The function of such an hypothesis, however, is not really explanatory, but simply an attempt to describe the facts of existence in the simplest possible terms. The immediate facts of existence are confused, complex, and loosely ordered. Any attempt to deal with them as they stand, for the purpose of calculated interference in the course of events, will be foredoomed to hopeless failure. Consequently, physics introduces such conceptions as those of a material particle and a luminiferous ether, in order to unify and co-ordinate the phenomena, so as to render them amenable to mathematical treatment.

The majority of hypotheses are merely descriptive in this way. They are attempts to describe the facts of existence in simpler terms than the immediately given data. It might therefore be urged that pluralism is also a merely descriptive hypothesis, the 'explanation' being simply taken back one step, and expressed in terms of different things. Yet it is just in this difference of terms that the root of the essential disparity between pluralism and other hypotheses is to be found. It implies a difference of type. For pluralism is expressed in terms of active selves. We all realize what it is to be active-it is just living and doing. We all realize what a self is. This realization is far more than knowledge in the ordinary sense. It is something of what the older thinkers were trying to express when they said that 
for perfect knowledge, knower and known must be one. It is a unique, supremely intimate fact, and therefore stands in a class of its own. It cannot be subsumed under one of the three types of knowledge properknowledge by acquaintance, knowledge by description, and knowledge of logical truths. It is this last fact which so often causes the realization of the nature and existence of self to be passed by, with the inevitable consequence that doubt is expressed of the existence of self at all. With a clearer view of the facts, such doubt is seen to be inherently meaningless. Moreover, it follows from the above that pluralism, being expressed in terms of active selves, is truly explanatory for such active selves, i.e., for us. It thus differs in type from all hypotheses which are not expressed in such terms.

Although pluralism differs in type from other hypotheses, it is yet bound by certain limitations common to all hypotheses. An hypothesis passes from necessarily partial observations of a system to a description of the system as a whole, and is therefore inevitably fallible unless the system be assumed capable of complete description in general terms. Any system, however, comprising subjects of experience, is quite incapable of being so described, for the subjects, and the experience of each, are essentially individual and unique.

It follows from the above that, as we have at our disposal but limited observations of the world, it would be possible to find an infinite number of hypotheses descriptive of the world, which would sufficiently fit the narrow range of the observed facts. We could not ${ }^{1}$ Evidently the subject or knower cannot be an object of knowledge. 
form a unique and infallible hypothesis ${ }^{1}$ unless we knew all the facts, past, present and future, and then it would no longer be an hypothesis, but a mere recital of those facts.

The fallibility of hypothesis is sufficiently illustrated by one fact alone, namely, that we have no reason at all to assume that laws which have held in the past will continue to hold in the future, unless we also assume some principle, such as that of induction, which depends on a priori principles of probability. Hence, though we may know an hypothesis to be false if it is contradicted by any fact, we can never certainly know it to be true. All that can be said is that it is more or less probable, the degree of probability depending on its applicability to the facts observed up to that time. Thus any final beliefs as to the constitution of the universe cannot depend on knowledge alone, but must be based on faith.

In selecting an hypothesis, then, we have a very great range of choice, for no hypothesis is ruled out of court till it fail to account for some fact, or rather, till it be definitely disproved by some fact. This being so, we naturally turn, in the first instance, to hypotheses which are truly explanatory. For our purposes an explanatory hypothesis may be formally defined as 'an account of a system which can be formulated symbolically in terms of active subjects of experience.' The conceptual formula as such, which sets forth the hypothesis, is, of course, descriptive, but its concrete meaning is explanatory in a sense in which that of a formula in terms of objective things is not.

1 I.e., we could not be sure that it was infallible nor that it was the only hypothesis which would fit the facts. 
It should be noted with reference to the type of result likely to be obtained from the scientific as opposed to the genetic method, that logical constructions of sense-data can never give a self. Hence, as selves certainly exist, no hypothesis in such terms can explain the universe nor even completely describe it. Pluralism, on the other hand, is not only explanatory, but it also complies with the condition demanded by Occam's razor. Far from multiplying entities, it is expressed in terms of entities certain examples of which we know to exist, and which any hypothesis must therefore take into account.

\section{Points of Conflict between Plukalism and THE 'NEW Realism'}

The supporters of the new scientific method hold that pluralism cannot be true because the conceptions on which it is based conflict with their results, and are therefore invalid. An analysis of the problem seems to show that the supposed conflict springs in the first instance from two main points of difference. These are the existence of the self, and the true meaning and validity of the categories of experience, particularly those of continuity and causality.

The scientific method lays stress on the objective side of experience. It investigates the object of experience, not in relation to the subject, but considered per se, and therefore in abstraction from the subject. It considers what meaning certain concepts must have if they are to be valid when applied to the object of 


\section{Points of Conflict}

experience thus isolated from the subject. Hence it fails to take account of the fact that the growth of experience consists in action and reaction between subject and object, manifested in an ever-increasing complexity and differentiation of the object, and that the latter is therefore determined in part by the activity of the subject. This activity is a fundamental realization $^{1}$, but when considered from the conceptual standpoint of Empirical Psychology, it appears to consist essentially in attention. If, then, we are fully to realize the concrete meaning of the concepts we apply to experience, we must examine them in the light of this mutual interaction of subject and object.

The consideration of the subject implied in experience brings in its train certain ethical and teleological concepts which are meaningless except in application to such a subject. The failure to take the existence of the subject fully into account in the analysis of experience, thus leads to the inevitable result that certain most important characteristics of existence are entirely overlooked or régarded as invalid conceptions. It is owing to their preoccupation with the objective side of experience that the New Realists look upon the notion of teleology, for example, with such doubt and suspicion. Bertrand Russell ${ }^{2}$ regards it as possible for a system to be both mechanical and teleological, according to the point of view. Such a supposition evidently entirely invalidates the generally accepted notion of teleology, and we shall therefore examine it hereafter.

${ }^{1}$ See p. 32 below, note on 'activity.'

2 "On the Notion of Cause," Scientia, Vol. XIII (1913), N. XXIX, 3 , p. 333 .

R. S. P. 
When concepts applied to experience are analyzed genetically, the meaning of them as thus determined is invariably found to contain more, and to strike deeper, than that determined by the scientific method. The former seems to throw considerably more light on the true nature of existence than the latter. This is illustrated particularly clearly in the case of causality.

Experience is a unity, comprising a duality of subject and object, and we cannot fail to get more and more out of touch with its true inwardness, if we lay stress on one side of it to the exclusion of the other ; for all separation of subject from object, though necessary to a certain extent for purposes of analysis, is to that extent artificial. The problem of continuity brings out most clearly, perhaps, the difficulties raised by this artificial separation.

\section{The Existence of the Self}

In the course of his analysis of our knowledge of the external world, Bertrand Russell makes the assertion that the (bare) self, if it exists at all, is an inference ${ }^{1}$. This sentence defines very precisely the general attitude of the new realists. It is somewhat as follows: In any case, the reasons we may have for stating that the self exists can only be arrived at by inference, but even then, it is doubtful whether the inference can be made.

As already pointed out, it appears that any such doubt of the existence of self is really meaningless. In the first place, from what may be called the concrete

1 Our Knowledge of the External World, Lect. III, p. 74. 


\section{The Existence of the Self}

point of view, we certainly cannot know the self from the very nature of the case; but we have instead the central and unique fact of the 'realization' of our own existence. Evidently no general term can adequately express the full nature of a fact so essentially particular; but this is no reason for ignoring the fact-perhaps rather the reverse. As will shortly be seen, we have in addition abundant data from which we can infer the existence of self, but the concrete realization of its existence is of infinitely greater importance.

From the more abstract point of view, psychology traces the gradual growth of the concept of self, from the primitive idea of the body-self, through ever more refined and spiritual stages. Eventually we seem to be coming in sight of the bare active subject of experience, as distinguished from the empirical self in all its phases. By proceeding in this way, however, we can never quite reach the subject of experience (though we may come very near it), for we are here dealing with self as conceived, i.e., as an object of knowledge; whereas the concrete self is the knower. Knowing is a relation between two entities, so that evidently the subject cannot know itself. It simply realizes its own existence, though the formulation in conceptual symbols of the fact of this realization, is itself a piece of knowledge. As Kant pointed out, it follows from the foregoing that the only course open to epistemology is to postulate the existence of the pure Ego, or subject of experience, as a regulative idea.

Although we cannot, by continually modifying, and, as it were, centralizing the concept of self, arrive at the concrete subject of experience, we may yet infer 
its existence. The inference is necessarily immediate, for all such terms as 'knowledge,' 'experience,' ' perception,' etc., imply the existence of a subject in their very meaning. Without it they have no significance whatever. In fact, all psychological discussion inevitably assumes the existence of an individual subject. We cannot speak simply of the existence of thoughts and feelings. There is always the implication of 'one whofeels and thinks.' Knowledge and consciousness only possess meaning at all in so far as they are referred to something knowing and conscious of something else ${ }^{1}$. Experience implies presentation of an object to a subject, thus comprising a duality in a unity. The existence of the subject in this duality is just as much a fact as the existence of the object. The Cogito ergo sum of Descartes was one of the most conclusive inferences ever stated.

Some philosophers, following Huxley, have regarded the self as being merely the series of mental phenomena constituting the individual mind. This supposition implies the existence of the very entity which it is attempting to dispose of. For, in the first place, what is meant by the 'individual mind'? Why should the series be individual at all ? What gives it its essential characteristic of unity? The fact of presentation to an individual subject is the only possible reason. Moreover, the very word 'phenomenon' implies appearance or presentation to something - to what we call the subject. It is useless to state, as some have done, that even if this be so, the subject may still be merely a logical construction, for this is to lose sight of the fact that the ${ }^{1}$ See also J. Ward, Art. "Psychology," Ency. Brit. 
agent which constructs can be no other than that subject which is supposed to be a logical abstraction. Finally, it should be noted that the exponents of the new scientific method continually use the word 'sense-data.' By so doing, they not only assert the existence of experience, but they also, by the very term, tacitly acknowledge that one element of experience is something which is 'given.' But if there be something given, there must be something else to which it is given.

To sum up: The existence of experience is a fact, and as such, cannot significantly be doubted. But experience consists essentially in the presentation of an object to a subject, and has no meaning which does not involve the existence of both the latter. The existence of the subject being once granted, propositions can be asserted concerning it. These constitute pieces of knowledge of a kind which has been termed 'knowledge by description.' Such knowledge is fundamentally distinct from that concrete realization of our own existence, which can in no sense be termed knowledge in itself, but which is, for us, the central and most abiding fact of all.

The existence of at least one self being granted, we proceed to assume the existence of other selves. This assumption is in accordance with the pluralistic hypothesis and is justifiable, for it in no way conflicts with the facts. It cannot be proved by the latter, however, so that it is an assumption; but it must be remembered that no philosophy can proceed without it. Solipsism is logically irrefutable, but quite barren. A man who is not a solipsist can prove a solipsist to be wrong to his own satisfaction, for he knows that he 
exists ; but he cannot prove to the solipsist himself that he is wrong. To all such attempts, the latter simply replies that the whole thing is merely part of a particularly vivid dream of his own. On the other hand, solipsism is equally unable to prove its case, so that we are at liberty to assume the existence of other people. This assumption is a most valuable one, for it at once opens to us an immense fresh store of knowledge by description, in addition to the knowledge we have through our own immediate sense-experience.

\section{Continuity}

Turning to the objective side of experience, we find in what is called the 'continuity' of experience, a source of difficulty which has been keenly realized by philosophers of all periods. The new realists claim to have disposed of the difficulties by means of their new scientific method, but the sort of continuity they are led to, is not the sort of continuity we find in experience, although, for most purposes, it may represent it adequately enough.

An examination of the disputes which have always centred round this question of continuity renders it clear that they are almost invariably rooted in the ambiguity of the term. Analysis shows that it is used with two very different meanings. There is first of all what might be called the older, common-sense meaning ; and then the modern, mathematical use of the term. The first may be best illustrated by considering the conception of a continuous material substance. Such a conception has appeared at various times and in various 
connections in physical science, as opposed to the atomic view of material substance. A continuous substance is structureless in the sense that it is not built up by the aggregation of a number of small elementary substances. Such a substance, though it seems paradoxical at first sight, would be indivisible; for the separation of an ordinary substance into two parts consists in overcoming the mutual forces between certain of its elementary particles. But in the case of our so-called 'continuous' substance, where there are no elementary particles, a moment's thought suffices to show that the operation of division could not actually be performed at all-all of which simply comes to this, that in such a case, when we say that our substance is continuous, we really mean that it is one-not relatively, but absolutely one and indivisible. In fact, the use of the word 'continuous' in this way is both arbitrary and unnecessary. Such continuity is just unity. Nothing more nor less than this is meant by the continuity of experience. The individual experience is an indivisible unity. The use of all such words as 'interpenetration' is simply the groping after the expression of that one fact-experience is one and indivisible. And, after all, what more do we need? There is no great difficulty in the conception of such a unity. It is one, because it is presented to one subject. The introduction of the additional notion of continuity is entirely gratuitous, and at once raises fresh and irrelevant difficulties. Much dispute and confusion would be avoided, if people would stop talking about the continuity of experience, and simply speak of its unity.

The modern tendency is rightly to restrict and make 
definite the use of the word ' continuity,' by employing it with one meaning only, viz., that of mathematical continuity. In this sense continuity is essentially a property of ordered series. The new realists suppose continuity of this kind to be typical of experience. Mr Russell', for example, asserts that the particular degree of continuity known as 'compactness' is sufficient to describe the continuity of experience. A compact series is one in which to any term there is no next term, that is one in which, if any two terms be selected, it is possible to find other terms between them. The number of terms of such a series is, of course, infinite. The view we are considering regards the objective side of experience as a compact series of sense-data.

The correctness or falsity of the view just stated hinges entirely upon the fact that a series consists of terms, and that however many terms there are, and of whatever magnitude they may be, they are discrete, each existing per se. Hence, if sense-data form a compact series, we must consider them to consist in an infinite number of scparate members, each of indefinitely short duration. So much seems to be admitted by $\mathrm{Mr}$ Russell. Yet again the point is overlooked that sense-data, though absolute and objective for the individual to whom they are presented, are relative and subjective from a universal standpoint. The separation of subject and object is still artificial. All that the theory under consideration has any right to assert is that the introduction of the notion of compact series is one of the most adequate ways of dealing with the unique continuity of experience considered 1 op. cit., Lect. V. 
objectively in abstraction. No doubt results based on analysis on these lines will be sensibly accurate when tested by experience; but this simply follows from the fact that the original constructions of compact series are sensibly accurate to the same order. It cannot be true, however, that experience is really composed in part of such a series of sense-data, for, as we have seen, the members of a compact series, in spite of their infinitude, are each a definite separate entity. The question might be raised as to whether such a series could have anything but an abstract existence. For example, we may write down any member of the compact series of rational fractions, but it is difficult to see how the complete series could exist concretely. Apart from this, however, we cannot look upon sense-experience as a compact series, for its continuity really consists in its unity. The totum objectivum of perceptual experience is one and indivisible, for it is presented to one subject. Hence if we analyze the former into a number of separate entities, we imply that the latter is also a series of separate existences. Again the appeal must be to concrete experience. The subject is one, persisting through change. This much we realize, though the notion of separate instantaneous existences may not be a logically impossible one. Moreover, it should be noted that the object of experience is in part determined by the activity of the subject. Again it is difficult to see how this could be so if there were sense-data existing independently as separate entities, and merely passively perceived in appropriate conditions. It would probably be replied that all that can be said is that certain motor sensations are followed by changes in the 
other sense-data, the motor sensations themselves being part of the data. But this assertion ignores the fact that the ground of the motor sensations is the activity of the subject.

The source of the whole difficulty, then, lies in the distinction between perceptual experience and its symbolic representation. Individual experience is unique, particular, and incommunicable. In describing it symbolically, therefore, our description cannot be entirely adequate, for it is conceptual, and the conceptual must always contain some element of the general. Hence the essential privacy of concrete individual experience cannot be comprehended in a descriptive formula. Moreover, in reflecting on experience and its implications, we are bound to attack it piecemeal, and to analyze it by abstraction, on account of our intellectual limitations. This inevitably entails a certain artificial immobilizing and dissection of experience, which we effect by means of concepts. Experience is dynamic and continuous, but concepts are static and discrete, even though they be concepts of things which are themselves dynamic.

The above point is illustrated particularly clearly by what $\mathrm{Mr}$ Russell calls the logical answer ${ }^{1}$ to the objections raised against the application of the mathematical theory of continuity to experience. Change, he urges, is a fact. But change involves relations, and relations are fundamental. Thus change demands analysis. Now we may grant that relations are fundamental, but what exactly does this mean? Simply that so soon as we come to analyze experience reflectively 
in abstraction, we find that we cannot proceed at all without the concept of what we call 'relation,' in addition to the concept of what we call 'thing.' Yet in the actual concrete individual experience there is no question of 'relation' or 'thing.' There is just a presented whole perceived by the subject, a whole which simply exists and is given as a whole. For example, let us try to imagine what may be called an 'instantaneous' section of experience. At any instant we perceive in fact but one object, the presented whole. No spatial series of separate parts (however great in number and however small in magnitude the latter may be) enters into the actual experience itself. The same is the case when we include time within our purview, and consider individual experience as a whole. There is here no temporal series of sense-data. Experience in its actuality is not a series. Considered in its entirety (which is the only adequate way of considering it) it is simply 'subject perceives object.' The object is an individual whole, and therefore, by its very uniqueness, cannot be characterized, as such, by such a general term as relation, for the latter implies the existence of more than one distinct individual. It is only when we come to reflect upon experience that we are bound to consider it piecemeal, and to introduce such general terms as 'parts' and 'relations.' To whatever closeness we may in this way approximate to the actual experience, we can never entirely get rid of that element of the general, which necessarily renders inadequate the conception of what is essentially particular.

$\mathrm{Mr}$ Russell makes the further statement ${ }^{1}$, that the ${ }^{1}$ op. cit., Lect. V, p. I 50. 
type of objection we have raised against regarding the continuity of experience as being of a mathematical kind is a particular example of a more general doctrine, which, broadly stated, amounts to saying that there can never be two facts concerning the same thing. $\mathrm{He}$ points out that the latter is evidently untrue. This may certainly be granted in general ; but how does it apply to the particular thing we are examining, viz., concrete individual experience? Strictly speaking, there is only one fact about such an experience in its actuality, which fact may be stated in the proposition 'It exists.' The 'it' of this proposition is the totum objectivum, or presented whole, of individual experience. Its actual nature is only realized fully by the particular perceiving subject. In actual perception, before reflection follows, it is perceived as an indivisible unity. It is often called the 'presentational continuum,' but it is more correct to call it the 'presentational unity.' All other propositions asserting facts (so-called) about experience, are simply attempts to express as adequately as possible in conceptual form the nature of private experience. They are inadequate, for the proposition expressing the sole and particular fact of the existence of the perceived object in its peculiar intimacy and uniqueness realizable only by the percipient, is replaced by a number of propositions, expressing our attempts to deal, by general characterizations, with something which is essentially particular. The attempts furnish us, for the most part, with a sufficient approximation, but in dealing with ultimate questions, it is of the first importance to remember that of necessity they are but approximations. 
It is evident, then, that the results of analysis by the scientific method cannot be fully adequate. This does not detract from the value of the former in practice, so far as it goes, for it is the most adequate conceptual method of dealing with experience. We could have no better conceptual way of representing what is called the 'continuity' of experience than by mathematical continuity. But this representation must not be regarded as a final complete solution, for the reasons we have given. It is necessary and sufficient for purposes of calculation, and for the establishment of the validity of certain physical conceptions, but the final solution of the difficulties which have been raised, lies in realizing that the so-called 'continuity' of experience is actually its unity, being, as it is, the experience of one subject.

What exactly is meant by 'one' thing? For example, we talk of a chair or a table as being one object; but the physicist regards the chair or the table as made up of a number of other single objects, viz., molecules. The molecules again, are made up of atoms, and so on. It appears that there are two distinct meanings to be attached to the word 'one' when applied to things. The first is subjective or teleological, the second objective and absolute ${ }^{1}$. It is in the former sense that a chair or a table is one object. In other words, we speak of a thing as being one, when it functions as one in relation to our purposes, or to the purposes of other subjects of experience. On the other hand, we can only regard a thing as being one in the absolute sense, when it is a

${ }^{1}$ We might, perhaps, distinguish a third use of the phrase 'one thing,' viz., as applied to a body which moves as a unit. [See Perry's Present Philosophical Tendencies, p. 52.] 
true individual. Where do we find such individuals? Evidently physics does not deal with them. Its molecules and atoms are not unique. Subjects and their experiences are the only true individuals. The self may be considered an absolute unit, for it is unique and indivisible. For this reason alone, pluralism, being expressed in terms of selves, would have an advantage over any description of existence in terms of sense-data, for the latter are, as we have seen, purely artificial units. Finally, it should be pointed out that the consideration of the meaning to be attached to the concept of 'one thing' is in no way connected with the meaning of the number 'one.'

\section{Causality}

Among the chief categories which are commonly regarded as applicable to experience, is the category of causality. This category has been a source of difficulty and confusion owing to the unfortunate vagueness and ambiguity with which the term 'causality' is frequently used, especially in its scientific application. Analysis by the new scientific method has done much to clear up this confusion. ${ }^{1}$. As a result of this analysis, it is pointed out that with regard to the objective side of experience, we can only say that the sequences observed in it are characterized by sufficient similarity to admit of more or less adequate description in general terms. Hence we can enunciate certain propositions in virtue of which the occurrence of some events can be inferred from the occurrence of other events. To these pro-

${ }^{1}$ B. Russell, op. cit., Lect. VIII. 
positions we may give the name of 'causal laws.' Therefore, from the purely scientific point of view, we should go no further than the mere statement that such causal laws do subsist. This is evidently true, for if we take objective experience as it stands, there is simply the fact that certain sense-data are invariably followed by certain other sense-data. The sequence contains in itself neither hint as to the reason for this invariance, nor warrant that it will continue to hold in the future. Scientific observation alone, then, can do nothing more than formulate descriptions of these sequences, together with the statement that it seems probable that they will continue to hold in the future as they have invariably done in the past. From this point of view, any further extension of the principle of causality is both unnecessary and unjustifiable.

If the exponents of the scientific method were content to stop at this point all would be well; but they go further, and assert that the meaning of causality considered above is the only valid one. Yet the roots of the concept of causality go far deeper than this. If we trace the development of this concept during the growth of experience, we find that it is inseparably bound up with the notion of efficiency or activity. We ourselves, as active agents, initiate changes in our environment, and we realize our activity to be the ground of which these changes are the consequence. Many of the sequences which occur in experience independently of us, we can reproduce at will. Thus we arrive at the conception of efficient causality as distinct from merely descriptive causal laws, ourselves being efficient, and, for the most part, self-determined 
causes. Inevitably we come instinctively to consider efficient causality as the ground of those sequences which we observe in experience. Logically, as we have seen, mere observation only gives us the right to assert that certain sequences do recur, and to state the fact in a general proposition. Seeing, however, that we actually realize self-activity to be the ground of many sequences-sequences which we can always render essentially similar-there is no reason why we should not adopt, at any rate hypothetically, the view that all observed sequences have their ground in the activity of experiencing subjects. In this way, causality, as applied to perceptual experience, comes to have a definite concrete meaning, namely, the efficiency of active individuals. No doubt the concept is anthropomorphic, but that simply means that it is based on the nature of the subject, as distinguished from the object, of experience. Hence we realize that efficient causality certainly exists, and we are therefore justified in attempting to find a satisfactory interpretation of the sequences which occur objectively in experience, by the application to them of this concept of efficiency.

Activity is fundamental. Everyone realizes what it is to be active. Yet certain modern representatives of the traditional idealist school $^{1}$, dismiss activity as

${ }^{1}$ E.o., F. H. Bradley in Appearance and Reality. The New Realists also reject activity (cf. R. B. Perry in Present Philosophical Tendencies, pp. 70, 99 and elsewhere). It is stated that all that is perceived is certain muscular sensations, etc., but no 'power.' This is not denied, but the fact (too often overlooked), which lies at the root of the question, is that activity is not an object of perception or knowledge at all. It is not presented to the subject, for it is the subject who is active. But we realize that we are active, although our 
pure illusion It is difficult to see what the assertion 'All activityis illusion' can possibly mean, if it mean anything at il. When I think or do, I say that I am active. All hat is meant by activity is a living and doing. If te idealist asserts that living and doing are illusions, th reply is simply that the illusion at any rate exists, ad therefore it is the illusion itself that we mean by acivity, if it be an illusion. There is no meaning at il in the term 'illusion' as applied to direct experience. $I t$ is only when wrong judgments are based on experienc that illusion can be said to exist. When we talk of bug active, it is simply a way of specifying a certain fac We may draw wrong conclusions from the fact, andin that case it is correct to say that we are the victirs of illusion. Yet, however that may be, the fact remins.

Returnin to the new realists, we find then that they deny the valiity of the concept of efficient causality. They maintin that the only meaning which causality can have is sat which we have seen it possesses in descriptive st:nce, namely, that in objective experience certain essenally similar sequences recur, which fact is expressed 1 a number of propositions (one for each set of simila sequences) termed 'causal laws.' This

activity is not prented to us. The realists and others might just as well deny the estence of perception, because we only find certain things given, of wich our own 'perceptivity' is not one. We do not perceive our pereptivity-it is not an object of knowledge-but we realize that we preeive things, and the proposition asserting this fact is of course a pie of knowledge (by description, not perception). Hence there is $\mathrm{n}$ more ground for denying the existence of activity in general, than if denying the existence of perception, in which the subject is active 1 a particular way.

R. S. P. 
they consider is all that can be said on the subject. As an example of a causal law the law of gravitation is frequently cited $^{1}$. The latter contains no reference to 'cause' or 'effect.' The expression of the law, as applied to a material system, simply takes the form of certain differential equations. From these it follows that the configuration of the system at any given instant is a function of that instant, and of the configuration at two given instants. This is true enough, but the fact remains that such differential equations, and the function which is their integral, are purely descriptive. They contain no hint as to 'how' and 'wherefore.' They simply tell us what does occur, without suggestions as to why it occurs. Moreover, there is still the question as to what determines the particular form of the equations from which the configuration at any instant can be deduced. It is not determined by logic, for logic and mathematics can give no answer to the question. As already suggested, the ground of the motions of such a system lies in activity. The particular nature of the motion, with its corresponding typical descriptive function, is determined by the particular type of activity of the agents concerned. The fact that our differential equations are shown by experience to hold for past and future as well as for present, simply means that the activities of certain individuals are sufficiently habitual to admit almost completely of description in general terms. We have seen that the introduction of the notion of active subjects does more than shift the descriptive formula one step

${ }^{1}$ See, e.g., B. Russell, "On the Notion of Cause," Scientia, p. 327. [See p. I 7 above, footnote.] 


\section{Causality}

further back, for it provides an explanation as opposed to a mere description.

In this connection Mr Bertrand Russell discusses the possibility that the universe may be a deterministic system $^{1}$. He considers a system of this kind to be one whose state at any time is given by a functional relation involving certain data which specify the state of the system at certain times, time being the independent variable. Such a functional relation is clearly equivalent to a description in general terms. But the universe contains unique individuals whose nature cannot therefore be exhaustively described in general terms. Even were such exhaustiveness possible in a relation of the type considered, the data contained in that relation would consequently have to comprise every individual in the universe, and the experience of each at every instant of his history. It is true that Mr Russell admits that the relation may be of strictly infinite complexity, but if it must necessarily be of the order of complexity we have indicated, it would simply be a recital of the whole history of the universe. That is, it would have to contain explicitly all the information which it might have been hoped to contain implicitly. There would, in fact, be no room in it for a variable at all, for it would contain all values of such a possible variable in its data. Hence, no such relation can be significantly applied to the universe in any ultimate sense 2 .

It is then urged against the notion of efficient causality that the future determines the present to the

${ }^{1}$ Cf. "On the Notion of Cause," Scientia, pp. 33 I ff.

2 This point is considered in detail in the essay on "The Notion of a Deterministic System." 
same extent as does the past; in other words, cause does not 'compel' effect, in some sense in which effect does not compel cause ${ }^{1}$. But, again, this determination of the present by the future is only logical and descriptive. Even assuming for the moment that certain functional relations actually subsist which are significant as a complete or partial description of such a Universe as ours, there is still a difficulty in the way; for although the formulation of the relations may work either way in time, we cannot ignore theone-directionality of time in concrete experience. The relations, though symbolic of a dynamic process, are themselves static. They simply assert that on given assumptions such as uniformity, there is logical dependence of the present on the future, just as there is logical dependence of the present on the past; but they fail to comprehend concrete experience fully, in that they ignore the actual fact that time progresses in one direction only. Thus, if all that these relations imply were true, there is no reason why the crime should not sometimes follow the punishment which is its due, nor why the determination to build a house should not follow the appearance of that house on the scene. Perhaps the existence of purpose and consequent action leading to realization illustrates best of all the hopelessness of the attempt to replace the notion of causal efficiency by the notion of mere logical dependence. For there is certainly a sense in which it can be said, for example, that the house was built because Jack determined to build it, in which it cannot be said that Jack determined to build the house because the house was built. Moreover, we are indu${ }^{1}$ B. Russell, Our Knowledge of the External World, Lect. VIII, p. 220. 
bitably aware that our actions determine their ends in a sense altogether different from that in which the ends determine the actions. This could not be so if the relation between them were purely logical.

The matter may therefore be summed up somewhat as follows: The true meaning which causality has for us is rooted in the realization of our own efficiency, as active individuals. The active individual is the 'cause.' The end which his (generally purposive) activity accomplishes is the 'effect.' The scientific method, however, takes the sequences which occur in experience as they stand and determines what may truly be said of them per se. In the first place, it finds that sequences continually recur sufficiently similar in nature to admit of a considerable degree of general characterization. Secondly, it follows that a general proposition may be affirmed with regard to each recurring sequence, whereby the occurrence of one event may be inferred from the occurrence of another event. Thirdly, there is no guarantee (except the rather doubtful one of probability) that such propositions will continue to hold in the future. Finally, it is seen that we can go no further than this from the objective standpoint of science. It might also be pointed out that, strictly speaking, the term 'causal law' ought not to be applied at all to such propositions as we have been considering. For, in view of the concrete meaning which 'cause' has for us, the word 'causal' implies that the sequences to which the propositions refer, have their ground in the activity of individuals?

The results of this analysis by the scientific method

${ }^{1}$ If this implication is granted, however, the term 'causal law' is of course appropriate. 
are valuable for the philosopher, for they make clear the exact nature of the assumptions he is making in applying the pluralistic hypothesis to the sequences observed in experience. Still more valuable are they for the physicist, seeing that they warn him from unwarrantable applications of causality, and point out the only valid way, from the scientific (and therefore descriptive) point of view, of looking upon the succession of phenomena with which he deals. There is no doubt that physicists of all times have been strongly influenced by the notion of causality based on subjective activity. One fact alone is sufficient to show this, namely, the curious reluctance which has always been shown to accept the idea of action at a distance. Attempts are invariably made to reduce everything to terms of contact action. The reason is that our own interference with the environment is conditioned by the contact of our bodies. Had we been endowed with powers of levitation and removal without contact, the notion of action at a distance would probably have been adopted as a matter of course.

Thus far, and in this application, we may recognize the truth and value of the results due to analysis by the scientific method. Pluralism, on the other hand, approaches the question in a different way, and with a different purpose in view. It is concerned not simply with the phenomena as such, but with an explanation of them which shall satisfy such beings as we are. On the basis of our own existence as efficient individuals, and of the fact that sequences observed independently of our activity can often be essentially reproduced by that activity, it proceeds to explain all sequences by the 
activity of individuals. This, of course, it is required to do if its hypothesis is to hold, and this it is successful in doing while no facts can be brought forward to disprove its case.

\section{ViII. Other Categories of Experience}

Although the consideration of continuity and causality brings out most clearly, perhaps, the distinction between the aim, method, and scope of pluralism and the new scientific method respectively, incidentally making clear the value to be attached to the criticism of the former by the latter, it is of great importance to examine the other categories of experience if a clear conception is to be framed of the basis on which pluralism rests. The attention may be directed in the first place to the categories of Substance and Attribute. A review of the classical attempts to deal with the notion of substance makes it clear that the problem resolves itself into an endeavour to reconcile the principles of permanence and change. Heraclitus, who was the first to bring out more or less plainly the nature of the difficulties involved, held that only change is permanent; but closer examination shows that, with any significant meaning which can be attached to the term 'change,' the truth of the matter is that change implies permanence. For, in the first place, it is apparent on general grounds that if there is a change, there must be a thing which changes, the said thing maintaining its identity throughout the change. Otherwise, there is simply one thing and then another thing, that is, mere succession and not change at all, properly so-called. From the 
scientific standpoint we certainly do consider mere alteration alone, that is, simply a succession of different presentations. But from the subjective point of view, if I have first $A$ and then $B$ before me, I can in no significant sense be said to have apprehended a process of change; at most there has been a change in myself, and this, since it is I who have perceived both $A$ and $B$, assumes my permanence. As a matter of fact, we do only perceive a process of change, as such, at a high level of experience; yet, when we have reached this level, we feel impelled to look for a permanent basis as a ground of the ceaseless flux of experience, whether it be logically necessary or not.

If we analyze the meaning of a process of change from a conceptual point of view, it would seem to be somewhat as follows: At a given time certain true propositions may be asserted of a given individual. At another given time, certain other true propositions, wholly or partly incompatible with the former set, may be asserted of the same individual. If we consider the propositions as particular values of certain propositional functions, the particular value considered of the argument of these functions remains the same throughout. This is the symbolic counterpart of the fact that the individual considered maintains his identity.

Evidently, from this point of view, it would be difficult, and perhaps impossible, to formulate in words the reconciliation of the principles of permanence and change. The reason for this difficulty is that, conceptually, we necessarily consider experience as a time-series. Let us attempt to estimate the true bearing of this. And here, perhaps, an analogy may be of use. We do 
not consider the identity of an individual at any given time to depend upon his position in space. At a given time, I should not be a different person if I were in London and not in Edinburgh. That is, identity is not conditioned by the spatial series ${ }^{1}$. Why, then, should it be conditioned by the time-series? It would probably be answered that the nature of the individual is different at different instants of the time-series. He develops (or the reverse) in time; and it certainly seems, at any rate prima facie, that time is more closely bound up with existence than space. But what is the time-series referred to? Not the conceptual or universal timeseries, for that is a mental construction. The private time-series of the individual concerned, then? But his time-knowledge is based on change and the existence of the memory-perspective, which implies maintenance of identity. Hence this line of thought bids fair to end in a vicious circle.

The truth is that we can never entirely resolve the difficulty conceptually for reasons we have considered in another connection, namely, that actual existence is particular and cannot therefore be comprehended in a conceptual formula. The conceptual formulation of the facts, if pressed too far, necessarily gives rise to diffculties which do not admit of complete solution. Nevertheless, it is possible to indicate to a considerable extent a method of viewing the facts which brings us as near as possible to a complete comprehension. In the first place, it is necessary to get rid of the time-bound view of experience. Just as in forming as adequate a con-

${ }^{1}$ I cannot, of course, be in two places at once, but that does not mean that I am what I am because of my position. 
ception as possible of the object of experience it is necessary to consider it as an indivisible whole in respect of space-time, so also must the individual subject of experience be regarded as a unity beyond space-time. In other words, we must try to conceive some such world as the universe of Minkowski. The latter applies his conception to the problems of physical science. In such a universe as he imagines, the entire existence of a physical system is specified by means of three spaceand one time-coördinate, and is presented as a whole. In a somewhat analogous way we must look upon the individual subject as an entity transcending space and time. His existence can only be specified as a whole; it is neither punctual nor instantaneous. From a logical standpoint, the proposition 'He exists' must not be supposed to imply any spatial or temporal reference; that is, there is no real meaning in the notion of existence at a given point or at a given time, though we may adopt the idea conventionally. The point is brought out still more clearly if we consider non-existential propositions which may be asserted of the individual. In examining this point previously, we pointed out that one set of propositions might be true at one time, and another partly or wholly incompatible set at another time. If, however, the propositions are modified by the insertion of date and place, their truth is independent of space and time. The date and place referred to may be considered as uniquely determined. For example, if they be specified by position in conceptual space and time, they will yet be connected by a one-one correlation with the private space and time of each individual. Hence there will be a set of propositions concerning 
the individual which will be true once for all. Regarding the propositions as particular values of certain propositional functions, the particular value of the argument of these functions is the individual considered. Since the propositions, however, are not limited as to their truth-value by space or time, the particular value of the argument cannot be dependent upon space or time. Thus the individual is a unity transcending space and time.

From this conceptual standpoint, such notions as 'process' and 'development' lose nothing of their meaning or value, but, like all concepts which refer to matter-of-fact, their inadequacy leads to the difficulties we have been analyzing. Yet although the solution of the problem of identity and change is attended by such difficulty when looked at from an abstract point of view, the concrete solution is more easily realized. The self combines, in a particularly complete way, the principles of identity and change. In spite of change, I realize myself to be the same individual that I once was. Even if we cannot formulate in words, on account of its uniqueness, the exact nature of this reconciliation of change and permanence in the subject of experience, it is, to say the least, almost as satisfactory to realize its existence. This being so, we are encouraged to apply the pluralistic hypothesis by regarding the permanent ground of the changing flux of experience as consisting in individual subjects.

It is important to comprehend clearly the general bearing of the view of space and time here adopted. Subjects of experience cannot be considered to be in any sense 'in space and time.' The latter phrase, indeed, 
signifies an abstract concept. Analysis distinguishes in the object of perceptual experience a succession of elements each of which has a sensible duration. The qualities and relations here implied are those to which we give the name 'temporal.' On them we base the concept of a time 'in' which the elements have their being. In a similar way, to the characteristics implied in the co-existence and sensible extension of the elements, we give the name 'spatial,' and hence pass to the abstract conception of space. But in the individual subject no such parts or elements, whether co-existing or succeeding one another, can be distinguished. Hence the subject is neither spatial nor temporal. It may perhaps be urged that we surely have a sense of enduring, which would imply time; but even if this be so, duration alone cannot give time. Our so-called sense of enduring simply comes back to the realization of our own existence, apart from any spatial or temporal implications. This is borne out by the consideration of any proposition, in which the subject is one term, having a spatial or temporal reference. In all such cases, a brief analysis shows that, so far as space and time are concerned, the assertion refers entirely to the object of experience. For example, the proposition: 'I remembered what happened to Smith, when I went to London last Friday,' simply asserts that there is in my object of experience (considered as a whole) a certain complex of motor, sensory, and ideational presentations exhibiting suchand-such spatial and temporal characteristics and relations. It would be hopelessly prolix, however, to attempt to make all our statements about the subject more exact by putting them in some such form as the 
above. For discursive exposition it is necessary to employ the usual shorter form, even though it appears to imply that the subject is himself in space and time. It may be well to remark, in order to anticipate any charge of inconsistency, that in all that follows the foregoing view is maintained ${ }^{1}$, and wherever statements (and they are unavoidably many) appear to relegate the subject to the realm of spatial and temporal entities, their use is simply an inevitable necessity if intolerable circumlocution is to be avoided. All such statements, however, are capable of exhibition in a form (as exemplified above) in which it is evident that any spatial or temporal reference is to elements in the object of experience alone.

Before considering the notion of 'attribute,' it may be of interest to make a short digression at this point, by referring back to Minkowski's conception of a spacetime world and its bearing on philosophy. The conception arose in the first instance out of difficulties similar to those we meet with in analyzing change. Recent researches in physical science have brought to the fore, with increasing insistence, the question as to what meaning, if any, is to be attached to such notions as absolute velocity and absolute position. The controversies to which these problems gave rise culminated in the enunciation of the well-known Principle of Relativity. There are several ways of stating the latter, but each amounts to this: 'Different descriptions of the same system will be given by different observers.' A description depends on the motion of the observer

${ }^{1}$ Cf. especially the essay on "Immortality," for a detailed discussion of the points raised here. 
relative to the system. There is no criterion which may be applied to a set of descriptions, by means of which a single true description may be determined. All the descriptions are true. The reason is that if we carry our analysis far enough, we are bound to consider the fact as a whole, namely, not only is there an object, but the object is seen by an observer. Consequently each perception is a different fact, and even admitting the object to be the same, for the purpose of argument, the descriptions, though all true, will be different, for each actually involves the observer and the observation as well as the object observed. Hence physics, which purports to describe things independent of any particular perceiving subject, is compelled in the end to take account of that subject. This is inevitable, seeing that the concepts of physics are constructions based in the first place on individual perceptions. In fact, the principle of relativity, as applied to physical science, is a particular example of the more general philosophic fact that while the experience of the subject is objective and absolute for him, it is subjective and relative from the universal conceptual standpoint.

In considering existence, then, from the conceptual point of view, we are continually brought face to face with its relativity. This is the root of the difficulty in the problem of change. As regards physical science, Minkowski succeeded in transcending the difficulty of relativity by introducing this idea of a space-time world. In this way he not only made clear the source of the trouble, but also indicated how it might be eliminated in analysis. It is simply a question of taking a wider view of existence; and in considering an individual who 
changes and yet maintains his identity, we shall get rid of the difficulties to a great extent if we proceed on similar lines. In specifying an individual, reference must be considered to be made to a space and timetranscending unity. In such a proposition as ' $A$ went to London on Saturday,' $A$ must not be supposed to be specified by any time or place. $A$ is an entity whose existence is considered as a whole. The proposition, though it contains a spatial and temporal reference, is asserted of this individual whole, which transcends both space and time.

In the existence of the self, then, the principles of permanence and change are reconciled; therefore the self is a concrete actuality corresponding to the concept of substance. A distinction is sometimes made between substances and Substance. The latter is regarded as some unity which is the ground of all existence. With the questions as to whether such a unity exists, and if so, what is its exact nature, we are not here concerned; but enough has been said to indicate that we can only form a satisfactory idea of such an all-pervading substance, by considering it to possess all the general characteristics of a self or subject of experience. Keeping, however, to the selves which we know to exist, and which we have identified with substances, what meaning ought we to attach to the term 'attribute' as applied to such individuals? In the first place, the term should be strictly limited. In particular, the fact that $A$ stands in a certain relation to $B$ must not be held to constitute an attribute of $A$. Propositions assigning attributes to an individual are of the subjectpredicate form. For our purposes, such propositions 
may be regarded as falling into two main types. These types may be illustrated by the two propositions: ' $\mathrm{He}$ is just,' and 'He is French.' The first makes a statement about the nature of the individual to whom it refers. The second, as such, asserts nothing directly about the nature of the individual, but is rather a specification of certain relations in which he stands. It should be noted, however, that this proposition may be held to imply a number of other propositions of the first type, namely, those assigning to the individual the characteristics he shares in common with all Frenchmen.

The term 'attribute' might well be limited to the predicate of propositions of the first type. A brief consideration suffices to show that such propositions invariably imply something about the mode of activity of the individual concerned. For example ' $\mathrm{He}$ is just' really means 'He acts justly.' We base our judgments about the individual on observations of his actions. Thus the attributes of the individual are the ways in which he acts. The fundamental proposition about an individual $A$ is ' $A$ exists,' which is equivalent to ' $A$ acts.' $A$ is a unique particular who cannot be further specified symbolically. What we call his attributes consist simply in his mode of activity.

Two categories which seem interwoven particularly closely with the fabric of experience are those of Quality and Relation. Quality and attribute are often used as synonymous terms, but to maintain precise definition we may distinguish between them. Just as we have taken the conception of attribute as appropriately applicable to the individual subject of experience, 
so may we appropriately apply the concept of quality to the object of that experience. For example, the sense-data presented through different organs differ in quality, e.g., sensations of colour differ from those of touch. There are also qualitative differences between sense-data presented through the same organ, e.g., red differs from blue. Differences of intensity are really qualitative too, although expressed quantitatively, thus implying a relation of more or less between the sensations ${ }^{1}$.

It is correct to say that Quality and Relation are fundamental in the object of experience, if the exact implication of the statement is clearly comprehended. In concrete experience, as such, there is no question of quality or relation. There is simply a given indivisible unity. This unity is particular, and can only be referred to by such words as 'it' or 'this.' Its characteristics cannot be specified conceptually with adequacy. We cannot take a single step in analyzing experience, however, without introducing the concepts of quality and relation. It is this which should be meant by the statement that quality and relation are fundamental. They are fundamental to the extent that we cannot reflect upon experience at all without introducing them ; but into the actual experience as such, they do not enter. This is evident when we remember that quality and relation are general conceptions, whereas experience is essentially particular. All we can say is that when attempting to represent experience conceptually (so far as it can be thus represented), by hypothetically con-

${ }^{1}$ For a detailed discussion of this point, see the essay on "The Intensity of Sense-Data."

R. S. P. 
sidering it to exhibit certain general characteristics, we find that two of the most indispensable of such characteristics are quality and relation.

Failure to realize the foregoing has been a fruitful source of objections levelled at the concepts of quality and relation. For example, consider $\mathrm{Mr}$ Bradley's criticism of the concept of relation ${ }^{2}$ on the ground that it implies an indefinite regress, seeing that a relation between terms requires further relations to relate it to its terms, and so on. There would be some point in this criticism if we asserted that the concept of relation adequately represents experience. But, admittedly; such general conceptions as quality and relation cannot adequately comprehend the essentially particular. All that is claimed is that in representing experience as adequately as possible by general characteristics, the introduction of the conception of objects between which certain relations subsist, is, for the most part, perfectly satisfactory for the purpose of calculated prediction and interference in the course of events. No such complication as the introduction of fresh relations between the relation and its terms is needed to carry on the reasoning based on our premises, and this reasoning is justified, so far as it goes, by empirical verification. Thus Mr Bradley's objection cannot hold good, for we do not suppose our conceptual system of terms and relations to comprehend experience fully, though on the other hand it is sufficiently adequate to describe it and to render possible sufficiently accurate

1 These are hypothetical in so far as we consider them to be absolutely identical elements in every individual experience.

2 Vide Appearance and Reality, 2 nd ed., Ch. III, pp. $3 \circ \mathrm{ff}$. 
prophecy and successful interference in the course of events. Therefore the objection has no significance as applied to perceptual experience as such, nor can it be urged against our conceptual apparatus; for we construct the latter ourselves, and find it sufficiently competent to perform its task, which is the only significant test.

We may conclude the investigation of the categories of experience by examining two of a somewhat different type from those already considered. They are the categories of Means and End, or Purpose. These categories are only significant in application to a universe containing individual subjects of experience. The categories we have been analyzing are applied in the first place to the object of experience, though the origin of the concept of the category is in some cases subjective, but the category of purpose is primarily applicable to the subject of experience, for it is a characterization of activity. It seems probable that all activity is originally purposive, though oft-repeated actions become less and less consciously purposive and more and more reflex and habitual. As regards those individuals whose nature we realize most clearly, namely, selves at our own level of development, the ground of activity is in most cases evidently purposive, and not purely material in the scientific sense. For in science, 'material' means 'phenomenal,' whereas the ground of our own activity is the very opposite of phenomenal. Certainly phenomena in part determine the purposes which guide the activity, and the latter may itself be limited by material conditions, but the ground of its initiation is subjective or real as opposed to objective or phenomenal. 
We may, however, attempt to apply the category of purpose to the ground of what we observe in the object of experience. In such observation we at once notice actions which may be regarded as purposive by analogy with our own. In fact all organic life appears to exhibit this purposive character. We might perhaps describe the activity of an organism in terms of molecular action, that is, in terms of the purely objective constructions of physics, though it is by no means certain that organic activity could even be completely described thus. In any case, the description, if complete, could not be general, for every organism is unique. Each would therefore require a separate description. On the other hand, we may explain the organism by the organized collective activity of individuals, thus changing the terms from purely mental constructions to concrete entities whose nature we can all more or less realize.

The fact that organic activity is thus apparently teleological strongly suggests the applicability of the pluralistic hypothesis, at all events to organic matter. We say 'apparently teleological,' for it is not certain that the existence of such teleology can be conclusively proved from a logical point of view. Could it be so proved the fact would be of enormous significance, for pluralism would immediately be verified as regards organic matter, since the existence of purpose implies the existence of experiencing subjects. However that may be, the attempt to describe organic life in purely physical terms invariably leaves an inexplicable residue of spontaneity, whereas its explanation in terms of individuals differing only in degree from ourselves, is 
enabled to take the latter fully into account. The pluralistic hypothesis is therefore to that extent justified.

Inorganic matter may be treated by a similar, but somewhat modified, theory. It is analogous to organic species which have become stationary at some period of their evolution. It approaches the lower limit of development. It may be regarded as comprising individuals of an extremely low order of mentality, who therefore exhibit the minimum of spontaneity and the maximum of habit in their reactions. They are thus particularly susceptible of an almost complete description in general terms.

It may be concluded that wherever the category of end or purpose can be successfully applied, so also may the pluralistic hypothesis be applied to the same extent; for the existence of purpose implies the presence of mind, that is, of subjects of experience. We have seen that pluralism is in this way applicable not only to the ground of what we term the organic activity observed in the object of experience, but also (with certain modifications which yet conform to the necessary conditions required by an explanation in terms of mind) to the ground of inorganic activity. The whole field of experience may therefore be covered by pluralism ; though we are here concerned, not with the details of the application of that hypothesis, but only with its basis. 


\section{Summary and Conclusion}

We may end our examination of the two most important tendencies in the trend of modern philosophic thought, by summarizing the results to which we have been led. In this way, the scope and limitations of each school of thought, and the results which each may hope to obtain, will be set out concisely and in brief compass.

The scientific method, as expounded by philosophers of the new realist type, is embodied in an analysis of the object of experience, with a view to ascertaining the form of the facts concerned. With the particular content of any set of facts it is the business of one of the particular sciences to deal. Philosophy aims at determining form, without reference to any particular content. This philosophic analysis has an important application in the investigation of those concepts which we ordinarily apply to the object of experience, notably the concepts of physical science. Since all observation, avowedly scientific or otherwise, must start from sensedata, and since all verification of calculation based on such observation must lie in an appeal to sense-data, it follows that if our concepts are valid, they must be capable of being exhibited as logical functions of sensedata. The analysis of the concepts, therefore, finally resolves itself into an attempt to build up such constructions of sense-data as may be considered satisfactorily to represent the concepts involved. Hence the method, at any rate in the last stages of its application, is constructive. Nevertheless, its function is evidently to a considerable extent critical. Its field of application consists in the whole of the objective side 
of experience, and we may willingly admit the claim of its exponents, that it is the only method of obtaining accurate objective scientific knowledge, provided it is clearly recognized that it is subject to two limitations springing from a common root. In the first place, all individual experiences are essentially particular, and the assumption that they exhibit certain general characteristics of form must therefore be regarded as an approximation which is only justified by the fact that it works satisfactorily in practice so long as we are not concerned with a final complete adequacy, and by the still more cogent fact that we are bound to it by our intellectual limitations. In the second place, the units with which the scientific method works are sensedata, and the sense-datum is a purely artificial and conventional unit. The object of experience is an indivisible unity, and (whatever convention we may be compelled to adopt for the purposes of calculation) cannot be considered to consist in a series of members termed 'sense-data,' compact or otherwise.

Keeping in mind these limitations of the method, its critical and constructive value in its own field is apparent. In any case, however, its results are purely descriptive. Its exponents claim that the determination of such results as their method affords is the only business with which philosophy ought to concern itself ${ }^{1}$. As the opinion of philosophers of all ages, with very few exceptions, has differed widely from this, the claim must be regarded as a purely arbitrary one. In making it, its supporters are proposing their own definition of philosophy, a definition which is not accepted by the

1 But see note on p. 7 above. 
majority of philosophers. In addition to the critical investigation of the form of facts, it is the further business of philosophers to provide an hypothesis which may be said to explain those facts to the satisfaction of such beings as ourselves, while remembering that, although no hypothesis can be regarded as infallible, it may be invested with a very high degree of probability, in virtue of its ability to fit the facts already known, and to furnish explanations of those new facts which are constantly forthcoming.

In applying the scientific method to the various problems of philosophy, the new realists have little or nothing to say about the subject of experience. Such brief references as are made imply that the subject, if it exists at all, is merely an inference. But, as we saw, doubt of the existence of the subject is without significance; and, moreover, although the existence of the subject may certainly be inferred-immediately inferred, indeed, from every single fact of experience-there is, in addition, the far more important central and unique fact of our experience, namely, the concrete realization of our own existence.

There are several important consequences of this ignoration of the subject. In the first place, certain problems are considered to be outside the scope of philosophy. Such, for example, are the problems of ethics. Mr Russell says: "The difference between a good world and a bad one is a difference in the particular characteristics of the particular things that exist in these worlds. It is not a sufficiently abstract difference to come within the province of philosophy'." Again 1 op. cit., Lect. I, p. 26. 
this limitation of the philosopher's task is a purely arbitrary one. The terms 'good' and 'bad' are only significant in a universe containing such individuals as ourselves. In their fundamental application they refer to the acts of an individual considered in relation to other individuals. Judging the individual by his acts, we may conventionally use the terms in reference to the individual himself. Further, we may also apply them to the object of experience considered in relation to the subject, thus introducing the categories of value. The latter, being categories, are necessarily concerned with the form of facts, thus coming within the scope of philosophy even as limited by the new realists. Moreover, even in application to the acts of the individual, the terms 'good' and 'bad' might be taken to refer to the form of the acts. For example, we might define 'good' as the class of all acts which have as their motive the benefit of others. In any case 'good' and 'bad' are general characterizations, and it is the business of philosophy to define their meaning precisely, and to determine their application.

The ignoration of the subject also leads to the consequence that the results of the scientific method are purely descriptive and not explanatory. It does not seek the ground of the object of experience. Men have always felt that there must be such a ground, regarding sensations, per se, as flimsy and ever-changing manifestations of a more substantial reality. In dealing with sense experience, we find it easy to distinguish and to compare, and generally to construct a complicated network of terms and relations. The facility with which we perform such conceptual gymnastics tends to make 


\section{Scientific Method in Philosophy}

us lose sight of the fact that the object of experience, as given, is an indivisible unity. When we turn to the subject, however, the case is different. We come down to bedrock almost at once. Any attempt to analyze the subject into parts and relations, at once shows the futility of regarding it in any other light than as a single unity. In the case of the individual subject, we are therefore concerned with content rather than with form. We find in it a substantiality which the object of experience seems to lack, for we are ourselves individual subjects of experience. We are thus led, with pluralism, to look for the ground of the object of experience in the activity of individuals differing only in degree from ourselves. Our own existence is for us the central fact of the Universe, and any attempt to limit philosophy to enquiry into matters where the existence of the subject may be safely ignored, on the ground that we must deal only with form and not with content, is both arbitrary and highly unsatisfactory. After all, the facts of the universe are particular, and it surely lies with philosophy to explain those facts so far as it can.

Every philosophic theory must necessarily assume certain logical axioms in accordance with which its reasoning is to be carried on. It is one of the principal tasks of modern logic to reduce such axioms to a minimum. Taking these principles of reasoning for granted, the theory will proceed to start from certain definite facts as data. The more incontrovertible and immediate the facts, and the more fully realizable, the more satisfactory is the theory likely to prove. Pluralism starts from the existence of the self. It makes the assumption 
of the existence of other selves. Thus it is based on the existence of entities at least one example of which we know to exist, and whose nature we actually realize. It is therefore superior at the outset to theories which start from entities such as sense-data that are objective for the individual. For, in the first place, all such objects are purely artificial units, whether they be sense-data, or the constructions of sense-data which constitute the units of the world of physics. On the other hand, a self is a true unit, a true individual. In the second place, we realize what a self is. We perceive a sense-datum, but we cannot realize what it is, in itself. Moreover, there is the further point that selves cannot be resolved into sense-data, whereas it may be possible to explain sense-data in terms of selves.

The next step in the development of pluralism is the analysis of the growth of the experience of the individual subject by the genetic method. It is not sufficient to enquire what certain concepts ought to mean, but also what they do actually mean for us, and how they come to acquire that meaning. If we proceed on these lines, particularly with reference to the chief categories of experience, we arrive at results which in each case, while not leading to it as a logical necessity, strongly suggest the pluralistic hypothesis.

The part played by the subject in experience is not a purely passive one. We find that we are able to interfere in the course of events, and, within limits, to guide the latter to the fulfilment of our ends. The realization of this ability is the basis of the notion we form of efficiency, and in it the root of the concept of causality is grounded. The concrete meaning of causality 
for us is therefore the efficient determination of one thing by another. This relation of efficient determination is one-directioned; it is not reciprocal. This follows from the fact of the one-directionality of time in actual experience. No doubt if we formulate symbolically this sequence of cause and effect, there is logical dependence of one on the other. Such a logical dependence, however, is descriptive, and does not alter the fact that in actual experience our activity determines its consequences in an entirely different sense from that in which it is determined by them.

In the course of his development, man comes to apply this idea of efficient causality to the sequences which he observes in the object of experience. The question then arises as to whether this application is valid. If we take the sequences simply as they stand, it is certainly not valid. We can only say that certain sequences do occur, and that we are able to formulate propositions in virtue of which the occurrence of some events can be inferred from the occurrence of others. From this purely scientific standpoint, causality is merely a logical and descriptive dependence of one event upon another. We may, however, wish to go beyond the mere existence of the sequences in an endeavour to find some satisfactory explanation of their existence as sequences. We know that some efficient individuals exist, and we also know that some of the sequences observed are initiated by the activity of these individuals. Hence we take as an hypothesis the proposition that all sequences have their ground in the activity of efficient individuals. This hypothesis is not logically proven, but it covers the facts by explaining them in 
terms of entities whose nature we can realize. It is therefore justifiable.

It is necessary for the purposes of reasoning to formulate our ideas in terms of sequences of sense-data. But the artificiality of the latter is brought out by the consideration of the problem of continuity. In fact, the problem itself is due to this artificiality. That fundamental characteristic of the object of experience which is commonly termed 'continuity,' is really unity. This consideration alone is sufficient to show that any theory which purports to give a final answer to any of the problems of the Universe in terms of such things as sense-data, may be ruled out at once. On the other hand, the unity of the object implies the unity of the subject, for this is its ground. It thus emphasizes the fact that selves are single entities that may be taken as true units in terms of which to express our explanation of the objective facts of existence.

This idea of the self as a unity which, in its completeness, transcends space and time, though for most purposes we conceive it as developing in space and time, is the conceptual representation of something realized concretely in actual experience, namely, the persistence of our identity through change and development. This reconciliation in the self of the principles of permanence and change provides us with a concrete example of that which we endeavour to conceive when we talk of 'substances.' It is impossible to formulate the reconciliation adequately in words, but it is there, and we realize its existence and its nature. We cannot rest content with regarding the object of experience as mere change (whatever that may be) based on no 
elements of permanence, so that we come to look upon experience as interaction between self and other selves, following the pluralistic hypothesis. Accordingly, the attributes of these selves or substances are their modes of activity.

In many cases this activity seems to lack spontaneity and to conform more or less completely to general laws, being due, as we suppose, to selves of extremely inferior mentality, and so, for the most part, the slaves of habit in their reactions. In many other cases the activity is only completely explicable with reference to the end which it achieves. Possibly we might be able to describe the activity completely in terms of the ordinary objective conceptions of physical science. This alone, however, leaves us far from satisfied. We can no more be content with it than we could be content with a mere description of the acts of other people accompanied by no statement nor understanding of their reasons for those acts. But if we regard all activity as being due to purposive individuals, we not only observe and describe the activity but we understand it. It acquires meaning, where before it was meaningless.

The new scientific method is, then, in its own field and for its own purposes, a most powerful weapon of research. For the ends it has in view, the ignoration of the subject of experience is justifiable; but this only so long as we remember that the results obtained must not be regarded as giving a fully adequate account of things, even on the objective side of experience alone, but simply an account which, in its proper application, is the most satisfactory that can be obtained, owing to the limitations of the conceptual standpoint. The 
ignoration of the subject has, however, the important consequence that the results obtained by the scientific method may not validly be used to criticize such an hypothesis as pluralism, for they stand on an altogether different ground. On the one hand we are investigating the logical form of facts, on the other we are out to explain the facts, and unless in doing so we describe the facts wrongly, we cannot lay ourselves open to criticism of the kind indicated.

The type of result afforded by the scientific method leaves most of us unsatisfied. We wish to go further than mere description. The pluralistic hypothesis is an endeavour to satisfy this wish. It attempts to put everything in terms of things whose nature we actually realize, and which may therefore be simply indicated without the necessity of formal conceptual specification. This is all the more important because such a specification can never adequately comprehend the object to which it refers. Pluralism is, of course, an hypothesis, and therefore subject to the limitations of hypothesis in general, but it is based on no assumptions save in so far as it makes use of the logical canons of reasoning, if those can be called 'assumptions.' The assertion of the existence of the self is not an assumption; and although we have referred to the assertion of the existence of other people as being an assumption, it is not so, strictly speaking, but rather the first step in the application of the pluralistic hypothesis to the explanation of the facts of experience.

So far as we are able to explain the facts by it, pluralism is therefore an eminently satisfactory hypothesis; for, while it avoids the introduction of unknowns, 
64 Scientific Method in Philosophy

it brings home to us the nature of existence in general in an entirely unique way. We have the assurance that where it is successfully applied, the result will be, not merely to shift the problem back a step, thereby creating a new problem of the same type, but to provide a final explanation-an explanation which is capable of fully satisfying such beings as ourselves, in the search for the true nature and meaning of reality. 


\section{II}

\section{ON CERTAIN CRITICISMS OF PLURALISM}

\section{INTRODUCTION}

$I_{\mathrm{T}}$ is incumbent on anyone who attempts to establish and develop a pluralistic view of the universe, to consider, and, if possible, to meet certain vital criticisms which have been urged against such a view. The answers to these criticisms must be prefaced by a brief indication of the standpoint from which they are approached.

The present writer regards a spiritualistic pluralism (essentially such, for example, as that maintained by Dr James Ward) as the most satisfactory hypothesis on which to base a system of philosophy. It is satisfactory, in the first place, on account of the fundamental conceptions from which it starts. These are perfectly definite and easily realized. Secondly, it affords a most promising method of attacking and of partially or completely solving some of the outstanding problems of philosophy.

In the course of the development of this hypothesis, however, it becomes clear that alone it is incomplete. This is to be expected, for the history of philosophy shows that no system can hope to approach within measurable distance of its object which lays undue stress on either of the dual aspects of the universe (its oneness and its manyness) to the neglect or exclusion of the other. 


\section{On Certain Criticisms of Pluralism}

We find, accordingly, that criticisms of pluralism fall mainly into two classes, those which demonstrate its incompleteness as a final answer to the questions which it seeks to resolve, and those which are aimed at supposed flaws radically inherent in the hypothesis itself. As has been indicated, the former may be regarded as justified, but the latter call for an answer, and it is with certain of them that we are here concerned.

Of the great philosophic systems of the past, the Monadology of Leibniz is perhaps the most remarkable for the logical skill with which it is sustained, and for the keen insight manifested in the fundamental principles on which it is based. From it all modern pluralisms derive their central theme. But two centuries of criticism have ensured the evolution of systems in which the more prominent weaknesses of the original monadology find no place. These later systems drew inspiration afresh from the great biological advances of the last century, advances made in the light of the doctrine of the evolution of species, a doctrine already foreshadowed in Leibniz' celebrated Principle of Continuity'.

Yet there remained in pluralism certain vulnerable points which its opponents were not slow to attack. With all the criticisms thus put forth it is both impossible and unnecessary to deal at length. The most important of them are to be found in the writings of two men: Prof. Pringle-Pattison ${ }^{2}$ and $\operatorname{Dr}$ Bosanquet $^{3}$. If the

1 The doctrine of pre-established harmony shows, however, that evolution, as we now understand it, did not enter into Leibniz' conception of the universe.

${ }^{2}$ In The Idea of God in the Light of Recent Philosophy.

${ }^{3}$ In The Principle of Individuality and Value. 
objections there urged can be successfully countered, the chief difficulties which block the path of the modern pluralist (not necessarily as regards philosophy in general, but as regards pluralism in particular) will be swept away. Accordingly, it is with the criticisms put forward by Prof. Pringle-Pattison and by Dr Bosanquet that we are called upon to deal.

\section{Externality}

For the pluralist, the environment of the self or subject of experience consists in other selves or subjects whose mentality differs from his only in degree. This belief is attacked by Dr Bosanquet in a criticism which may be summed up essentially somewhat as follows: "[Selves] as inward centres in the popular sense [cannot] form the circumferences for each other ${ }^{1}$," and again, "Even if there were, de facto, a psychical something underlying matter, yet it is only as definite externality that it plays a part in our life. We have no use for it as inwardness"."

Now the true implication of these sentences is by no means evident if we inspect them as they stand. The spatial metaphor involved in the use of such words as "centre," "circumference," "inwardness," "externality," tends rather to obscure the issue, though the introduction of that metaphor may be very convenient and to a certain extent necessary. But what does this distinction between "inwardness" and "externality" really imply?

${ }^{1}$ See, e.g., op. cit., pp. $75 \mathrm{ff}$.

${ }^{2}$ Ibid., p. 194, note. These quotations summarize the idea involved and explained at length. 
Evidently "inwardness" is something which essentially characterizes the individual subject, at least for that subject, whereas "externality" is something which characterizes (for him) the not-self. Hence the distinction between "inward" and "external" refers ultimately to the fundamental distinction within each individual experience of subject from object. Consequently, if the pluralist asserts that the object of experience of one subject consists of other subjects, Dr Bosanquet's criticism becomes in effect, "How can a subject of experience be, in any circumstances, an object of experience?"

In this form the criticism is justified, and the pluralist is wrong if he asserts that to any subject other subjects are presented as objects of experience. Before considering the latter point, however, it should be noticed that in any case the criticism only applies to pluralism incidentally. At the root of it is the fact that no existent entity can be an object of experience. No entity other than myself can begiven to me as an object of knowledge in such a way that I realize what it is in its actual essence. ${ }^{1}$. We cannot in experience know anything else as it really is in itself.

What, then, of the sense-data which form for each individual his object of experience? They are objects of acquaintance-knowledge. Are we to say that they do not exist? Strictly, it is neither true nor false to say that they exist. It is meaningless. There is no significant sense in which existence can be asserted of

1 I do not mean to imply here that even the self is given as an object of immediate knowledge in experience. This point is dealt with more fully in the previous essay, Section V. 
the immediate data of perception. There they are, and that is all that can be said of the matter. Accordingly we must regard the object of experience not as one or more existent entities, but as the "appearance" to the subject of existent entities other than himself. This fact of "appearance" or "presentation," being ultimate in nature, defies satisfactory definition. It might be provisionally indicated somewhat as follows: Given a percipient subject and certain other existent entities, under suitable conditions, of which the existence of these other entities is the most necessary and important, the given subject will perceive an object which may be defined as the "appearance" to him of the other entities. It is important to notice that this "appearance" is neither the given subject nor the other entities, though its being is dependent on the existence both of the subject and of the other entities'.

Prof. Pringle-Pattison ${ }^{2}$ also makes a brief reference to the point under consideration. He remarks that "internality is impossible without externality." This, as we have seen, is equivalent to saying that a subject of experience is inconceivable apart from a presented object of experience. But the latter is simply the appearance to the subject of other existent entities. It is not itself to be classed as an existent entity, though it has being in the sense that it is there. A subject, however, to whom no appearance is presented is just as inconceivable as an appearance presented to nobody.

${ }^{1}$ For a fuller treatment of this point see the next essay, end of Section III, and also the essay on "The Relation of Mind and Body," end of Section II.

${ }^{2}$ Op. cit., pp. $178 \mathrm{ff}$. 
It follows, then, that Dr Bosanquet's criticism does not apply in any special way to pluralism, but is really an expression of the fact that an existent entity cannot be an object of knowledge. In particular, an experiencing subject cannot be an object of knowledge. But pluralism is in no way bound to assert this impossibility. For pluralism, the living experience of the subject consists actually in his interaction with other subjects. This interaction is manifested in the ever-increasing differentiation of a presented indivisible whole or object of experience, namely, the appearance to the subject of other subjects. We are not acquainted in sense-experience with other individuals in their actuality. Selves cannot be reduced to sense-data. The latter are but what we have termed the "appearance" to us of other selves.

We may conclude our reply to this type of criticism by briefly considering another quotation from $\mathrm{Dr}$ Bosanquet. In pan-psychism, he asks, "what becomes of the material incidents of our life? ... Is it not obvious that our relation to these things is essential to finite being, and that if they are in addition subjective psychical centres their subjective psychical quality is one which so far as realized would destroy their function and character for us ${ }^{1}$ ?"

Now the nerve of this criticism is destroyed, as before, when it is realized that for a given subject the object of experience does not consist in a number of other "subjective psychical centres," but in the appearance to the given subject of these other subjects. Moreover, the function of material incidents in our life consists in the determination and limitation of our ${ }^{1}$ Op. cit., Lect. X, p. 363 . 


\section{Externality}

purposive activity. It is simply the manifestation of our interaction with other subjects. In fact, it is here that the fundamental ambiguity of $\mathrm{Dr}$ Bosanquet's term "inwardness" as a characterization of subjective centres becomes completely evident. For the activity of the subject is essentially "outgoing" as it were. It is not directed in upon itself (if that could have any definite meaning), but out towards others. How, then, is it possible that the development of this psychical quality can destroy the function of the subject with regard to other subjects? The growth of experience, in the pluralistic view, does not and cannot consist in a gradual withdrawal into itself of the subject, culminating in a complete isolation, but in continuous interaction with other subjects which, so far from leading to individual isolation, aims rather at mutual co-operation in ensuring the interests of the society as a whole.

\section{Consciousness}

Dr Bosanquet's conception of consciousness is in entire conflict with the position which pluralism takes up. But his view is largely vitiated by the fact that he adopts on this point an attitude which appears to tend very strongly to that Cartesian dualism of mind and matter, which for so long clogged the progress of philosophic thought. This tendency is particularly evident in his treatment of the relation of body and mind. The pluralist, on the other hand, recognizes that the fundamental fact from which the start must be made, is not a dualism of matter and mind, but the unity of the individual experience, which comprises a 
duality of subject and object. For the pluralist "mind" is a generic term denoting the class of subjects of experience.

According to Dr Bosanquet "organic regulation is natural and immanent, but independent of consciousness ${ }^{1 . "}$ Consciousness is a "perfection" granted by the Absolute in certain circumstances". Such statements imply that matter is given as prior, while mind only supervenes at a certain stage of the development of matter. This seems to approach perilously near to the epiphenomenal view. Moreover, if we grant with $\mathrm{Dr}$ Bosanquet that organic regulation is "natural and immanent," what evidence have we that it is "independent of consciousness "? Apparently the reference here is to the fact that the behaviour of an organism (especially of a lower type) consists largely in reflex action. The question is then whether the establishment of reflex action presupposes mind or not ${ }^{3}$. Now we have an abundance of examples of such a presupposition-a simple case being a man learning to ride a bicycle. In fact the formation of habits is a fundamental characteristic of mind. On the other hand, there are no cases in which we observe the establishment of a reflex action where we can infallibly assert the absence of mind.

It is the essence of the pluralistic position to recognize that the start must be made from individual experience,

1 Op. cit., Lect. V, p. 195.

2 Ibid., p. 189.

${ }^{3}$ Of course it is a well-known fact that established reflexes occur without the intervention of the dominant consciousness of the organism, but it by no means follows that the latter played no part in the original establishment of the reflex, nor that, even when established, the reflex is independent of any consciousness. On all these points see also J. Ward, The Realm of Ends, 2 nd ed., pp. $462 \mathrm{ff}$. 
which implies mind. It is the task of the pluralist to interpret matter from this standpoint. On the other hand, if we start from matter, how can we interpret mind? There is nothing in what Dr Bosanquet says on the subject which provides a satisfactory answer to that question. But from the standpoint of mind there is no such difficulty in interpreting organisms, at least. The striking feature of an organism is the fact that it exhibits "behaviour" analogous in every way to our own. Hence, what the subject distinguishes within its objective experience as organisms are, for the pluralist, the appearance to the subject of other subjects differing from himself only in degree or in kind of mental development.

Speaking again of consciousness, Dr Bosanquet says that "conscious process is meaning (or appreciation), not effect, of physical process " - and in another place: "Mind is the meaning of externality, which under certain conditions concentrates in a new focus of meaning, which is a new finite mind ${ }^{2}$." It is not easy to assign a definite significance to these assertions. In the first place "meaning" and "appreciation" are by no means synonymous terms. They apply respectively to the objective and the subjective aspects of the process which consists in the interpretation of an object by an individual subject. In other words, we regard the subject as "appreciating" the "meaning" of the object. It is difficult to see in what sense, if any, consciousness may be considered as "meaning." For the latter term implies both an object and a subject for whom the

${ }^{1}$ op. cit., pp. 196 ff., margin.

2 Ibid., App. II to Lect. V, p. 220. 


\section{4 \\ On Certain Criticisms of Pluralism}

object has meaning. We cannot regard the subject as being a "meaning." If we attempt to do so, we are bound to imply a further subject, and are thus led into a continuous regress. Moreover, Dr Bosanquet fails apparently to distinguish clearly between sensations and the mind of which they are the sensations. It is not clear whether the mind or the sensations constitute the meaning of physical process'. But, at all events, we cannot suppose mind to be simply the "meaning" of something else. "Meaning," though it implies a subject, is not itself that subject. Nor does it help us to adopt the term "appreciation" instead. For the subject is not the appreciation, but the individual who appreciates.

The conception of a mind as a "focus" of externality also appears to have no valid significance. As we have seen, the only legitimate meaning that can be given to the term "externality" is "the objective side of experience." But we cannot possibly conceive the subject as consisting in the "concentration" of sense-data into a "focus." To use Dr Bosanquet's terminology, internality can in no way be constructed out of externality. The term implies the fundamental distinction in experience between subject and object. We might perhaps speak (very loosely) of the subject as concentrating externality, by his unifying activity, into a focus. But externality thus focussed would be the product of the subject's activity and not the subject himself.

1 Even here there is a difficulty. For, as we have seen, a subject cannot be an object of knowledge, and anything which has "meaning" for anybody must in some sense be an object of knowledge.

2 Op. cit., p. 197 . 


\section{The Evolution of LaW}

In the type of pluralism advocated by $\mathrm{Dr}$ James Ward, the laws of inorganic matter, commonly called the "Laws of Nature," are regarded as having evolved in time, only reaching their present fixed and stable form after a long process of development. Prof. PringlePattison raises objections to this view. According to him we cannot suppose the possibility of action without environment, nor can we conceive the interaction of monads, even in the beginning, apart from laws in accordance with which that interaction takes place. And again: "A system of unvarying natural order is demanded, it may be pointed out, in the service of the higher conscious life itself, as the condition of reasonable action"."

Now, in the first place, it may be admitted that action is impossible without environment. But pluralism does not deny this. The environment of a monad is constituted by the other monads, with which it interacts. And, coming to the further point, Prof. Pringle-Pattison is evidently right in so far as he asserts that the monads must always have had some nature. But by the evolution of natural laws, the pluralist simply means that the laws of nature did not always exist in their present relatively fixed form. It must be remembered that such laws are not, as it were, imposed upon things from without, but are merely descriptions of the way in which things behave. Consequently, if the behaviour is modified, the descriptions or laws are correspondingly

1 Op. cit., pp. $183 \mathrm{ff}$.

2 Ibid., p. 187 . 
modified also; though in certain cases behaviour may tend to a comparatively fixed system of habitual reactions, in which cases we may speak of a fixed law.

The attitude of pluralism on this point may, perhaps, be made clearer by an illustration. In the first place it must be noted that, for the pluralist, there is no absolute gap between organic and inorganic matter. Now if we survey the realm of organic matter, past and present, we find that whereas some species continue to develop into more and more complex types, others have, after a long period of development, eventually approached a stationary condition in which their actions have become practically entirely habitual and relatively fixed in nature. Inorganic matter may be regarded as an extreme form of such stationary species. Hence there is no difficulty in supposing that inorganic matter has evolved into its present condition, and it is in this process that the evolution of the so-called "laws" of matter consists. There is obviously no reason to suppose that a limit must be placed on the number of these laws. Hence we may consider that originally each monad, while displaying the general characteristics of mind in a low degree, was yet, in its particularity, a law unto itself. Only as interaction proceeds is there a tendency for individuals en masse to behave in similar ways. This tendency proceeds from the characteristic, which must be present in some degree in each individual, of learning by experience.

As to what Prof. Pringle-Pattison says of the necessity for a system of unvarying law as the condition of reasonable action in higher conscious life, it certainly seems probable that the tendency of the individuals 


\section{The Evolution of Law}

composing inorganic matter to develop a system of habitual reactions has greatly aided the process of evolution of other individuals to higher and more complex types. Yet it must not be forgotten that each of us has to deal not only with material objects but also with persons. Although the behaviour of the latter does not admit of description to a degree of precision in any way comparable with such principles as the law of gravitation, for example, yet we do not find it impossible to live a rational social life on that account. In dealing with individuals whose behaviour is subject to continuous modification and development, the only necessary conditions of success are that the process of development should not be too rapid, and that we should have a knowledge at least of the general trend of that process. Such knowledge would itself be embodied in a law, but of a different type from those we consider in general under the conception of the evolution of law. For it would be the description of a dynamic process and not of a static form of behaviour.

It is evident, then, that the notion of the laws of nature as evolving gradually into their present stable form is not a contradictory one. For the evolution of law means nothing more nor less than the gradual modification of behaviour. We have examples in plenty of such modifications, and we find that in many cases the process tends asymptotically, as it were, to a limit, and we have species, which, after developing through countless ages, become relatively fixed. Relatively, we say, for there is no guarantee that even the laws of inorganic matter will, after the lapse of future vast periods of time, remain in their present form without sensible alteration. 


\section{The "BARE" Monad}

All mental life of which we appear to have clear evidence is associated in every case with an organism. The pluralist conceives the organism as a system of monads in association with a dominant monad, the latter constituting the self of which the organism is the body. But if we press the pluralistic hypothesis far enough, we seem bound to postulate, somewhere or somewhen, the existence of "bare" monads, i.e., monads unassociated with any body or organism. Prof. Pringle-Pattison points out objections to this view ${ }^{1}$.

Leibniz endeavoured to avoid the difficulty by assuming that every monad was associated with an organism composed of relatively inferior monads. For him, a piece of inorganic matter was a mere collection of organisms. In this way he piled infinity on infinity. We cannot be satisfied with such an endless regress. Nor does it really clear away the obstacles in any very definite manner, for it is difficult to see how, in considering the relations of organisms external to one another, we can entirely avoid the notion of the interaction of bare monads.

But, in any case, there seems to be no intrinsic difficulty in the conception of a bare monad. There is apparently no inevitable reason why that peculiar complex of presentations ${ }^{2}$ which constitutes what we call "the body" should enter as an element in every experience. A bare monad would simply be a subject from

1 Op. cit., p. 188.

2 Not only of sight and touch, but also that mass of organic sensations which constitutes what is called "general sensibility." 
whose object of experience this element was absent, and there is no way of showing that its absence is an impossibility. No doubt there is a difficulty of another kind, if we try to hark back to the monads as they originally were. For there is bound to be a difficulty here, but it lies, not in the notion of a bare monad, but in the inherent incompleteness of the pluralistic hypothesis. We are faced, in short, with the problem of Creation, which pluralism alone is powerless to solve. Yet one word of warning is necessary. Prof. PringlePattison seems, in one place, to identify the bare monad with what lies behind the atom, or whatever the ultimate physical particle may be ${ }^{1}$. This is quite unjustifiable. Physical objects, whether they be common-sense objects such as chairs and tables, or entities such as atoms and electrons, are conceptual constructions based on senseexperience, and therefore have a purely formal existence $^{2}$.

If the truth be told, the bare monad is not the real root of the trouble at all; the latter must be sought rather in the conception of interaction between the monads-and this applies just as much when the monads are members of one organism as when they are not. We need some concrete ground of this interaction, which shall serve as a principle of unification whereby the existence of selves forming a plurality, and yet entering into relations with one another, may be rendered

1 Op. cit., p. 180.

2 "This table" and "an atom" are alike capable of being exhibited as logical constructions of sense-data, though the latter is a more complex construction than the former. See B. Russell, Our Knowledge of the External World, Lects. III and IV. 
intelligible ${ }^{1}$. Although the start must be made from a plurality, and although the pluralistic hypothesis will carry us a long way in the understanding of the world, we must take account at the latter end of that other aspect of the world-its unity. With the further consideration of this question we are not here concerned. Suffice it to say, as in the introduction above, that such limitations of pluralism as are implied in this matter may be freely admitted.

\section{Summary and Conclusion}

It would appear, then, that the most important criticisms recently directed against pluralism fail of justification. We saw, in the first place, that there is no more difficulty in accounting on the pluralistic hypothesis for what Dr Bosanquet calls "externality," than on any other hypothesis, provided that we interpret that term correctly. It can only mean the object as distinguished from the subject of experience. For pluralism, the object of experience does not consist of other subjects (as Dr Bosanquet's criticism implies), but of the appearance of these other subjects to the individual subject considered, where "appearance" is defined in some such way as we have indicated. These "appearances" cannot be said to exist, for no existent thing can in itself be an object of knowledge, though they have being in the sense that they are there.

Secondly, Dr Bosanquet's account of consciousness does not agree with the facts. We have no reason

${ }^{1}$ Cf. below, pp. 203 f., and p. 250. 
whatever to assert that organic regulation is independent of all and every kind of consciousness. On the contrary, wherever we can observe the formation of a habit culminating in reflex action, it is associated with mind. Thus, whereas we have instances of reflex action presupposing the existence of mind, we have no instances of such action where mind can be certainly asserted to be absent.

We cannot construe consciousness merely as the meaning of externality. Such an interpretation is inherently contradictory. For, using the term legitimately, we speak of the "meaning" of an object for a conscious subject. We cannot significantly regard the meaning of objects as actually consisting in conscious subjects. Nor can we look upon externality as gathering itself up into foci which we call conscious subjects. No such attempts to get the subjective out of what is essentially objective can possibly succeed. Externality is not the less externality because it is concentrated into a focus, if for the moment we allow such a loose and metaphorical phrase. By no manipulation in this way can we make "externality" pass over into "internality" or mind, though we may perhaps look upon the latter as the agent which focalizes externality, provided we interpret our terms properly.

Proceeding to Prof. Pringle-Pattison's criticisms, we saw that one mistake lay in the misinterpretation of the word "laws." We cannot suppose that in Nature there existed laws and individuals as separate entities, and that these laws were then imposed on the individuals. By a natural law we can only mean the description of certain modes of behaviour. Consequently the evolution 
of law is nothing but the modification of behaviour, a matter of everyday occurrence. Occasionally a species becomes relatively fixed, in which case "the law" has evolved into a stable state. Inorganic matter may be regarded as providing extreme examples of such fixed species. No doubt we must postulate that even in the beginning the behaviour of each monad conformed to very general laws, though the behaviour of each would contain unique characteristics; but that is no reason why behaviour should not be modified, with the corresponding modification of descriptive laws. In short, no one wishes to deny the subsistence of laws, but merely to assert that laws may, and do, change. We do not start with fixed species. They are the result of long periods of development. Consequently there is no difficulty in supposing that the laws of inorganic matter have arrived at their present form after a lengthy process of evolution.

Finally there remains the question of the bare monad. This brings us very close to the limits of pluralism, and hence exhibits its incompleteness. For while there seems to be no inherent contradiction in the notion of a bare monad, it leaves us unsatisfied, since it directly involves the problem of the interaction of monads. We seek further for the concrete ground of this interaction, and are thus led to realize that some all-pervading principle, if it may be so called, is necessary to explain the unity of what in another aspect is a manifest plurality. There we must leave the matter for the present. If we are to achieve anything we must start from the given plurality of individuals, and this pluralism will carry us far. As we have seen, the difficulties supposed to lie in its way 


\section{Summary and Conclusion}

\section{3}

are by no means so real as they seem. But when the pluralistic hypothesis has done its utmost, we are bound to supplement it by a further principle, wherein we take account of that bond, whatever it may be, which makes reality a Universe. 


\section{III}

THE PHILOSOPHICAL PROBLEM RAISED BY THE WEBER-FECHNER LAW

\section{INTRODUCTION}

$T_{\text {HE well-known psychological law of sensation-differ- }}$ ence originally discovered by Weber, and subsequently interpreted by Fechner, is commonly considered to lead necessarily to certain important conclusions. The belief that these conclusions are inevitable consequences of the law is very widely held. When, however, the law is carefully analyzed, it will be found that the conclusions generally drawn from it are by no means the only possible ones. Moreover, upon the decision as to which conclusions are correct, there depends a philosophical issue of the first importance.

The Weber-Fechner law may be stated as follows: "The degree of unlikeness between two sensations is equal to that between two other sensations, when the ratio of the intensities of the stimuli producing the first two is equal to the ratio of the intensities of the stimuli producing the other two." The most important application of this law, and the one with which we are concerned, is to the case of small differences of sensation and stimulus. In such a case the law asserts that the least increase in the intensity of the stimulus which will produce a just "discernible" difference in 
the sensation, is directly proportional to the intensity of the stimulus. From this it follows that a finite difference of stimulus is necessary to produce the least "discernible" difference in the corresponding sensation.

The conclusion generally drawn from the above facts is that the sensation varies continuously with the stimulus, but this variation is not always perceived by the subject to whom the sensation is presented. This conclusion is made use of in many connections; for example, in the theory of subconsciousness ${ }^{1}$, and in the modern realist conception of experience as consisting in compact series of sense-data ${ }^{2}$. The theory of the subconscious can probably be formulated satisfactorily without making use of the conclusion referred to; but if the falsity of that conclusion should be demonstrated, the realist theory of experience just mentioned falls with it.

The reason generally given for affirming the conclusion that differences of sensation do in fact exist when no difference is actually discerned, is as follows : It is well known that the sensation corresponding to a stimulus $A$ may be indistinguishable from that corresponding to a stimulus $B$, and the sensation corresponding to a stimulus $C$ may be indistinguishable from that corresponding to $B$, whereas the sensation corresponding to $C$ is distinguishable from that corresponding to $A^{3}$.

${ }^{1}$ See, e.g., James Ward's article "Psychology" in the Ency. Brit.

${ }^{2}$ See B. Russell's Our Knowledge of the External World, Lect. V, pp. 14 I ff.

${ }^{3}$ E.g., see Stout's Manual of Psychology, 2nd ed., Bk II, Ch. I, § 3 . Also B. Russell, op. cit., Lect. V, p. 14 I. 
Hence it is argued that in each of the first two cases there must actually be a difference of sensation although this difference is not perceived.

\section{Examination of Conclusion drawn from the WEBER-FECHNER LAW}

In examining the conclusion thus reached, and the facts put forward in support of it, it will be simpler to employ physiological terminology in the first instance; that is, we shall continue for the present to speak in terms of "stimulus" and "sense-datum," although the concept of a stimulus is derived from our immediate data, and is not one of those data. The term "sensedatum" is a more satisfactory one than "sensation," for the exact meaning of the latter is somewhat vague. In its general usage there is no clear distinction made between what is sensed and the act of sensing, whereas the meaning of "sense-datum" is definitely confined to the former. It is with sense-data as thus defined that we have to deal.

Returning to the case of difference of stimulus unaccompanied by any observed change of sense-data, we may notice first of all that the facts must lead to one of two results. If a series of stimuli, $A_{1} A_{2} A_{3} \ldots A_{n}$, of gradually increasing intensity, is of such a nature that there is no observed difference between the sensedata corresponding to any consecutive pair, it follows that if the stimuli be applied in order, then either the sense-datum corresponding to $A_{n}$ is indistinguishable from that corresponding to $A_{1}$, or, if this is not so, a difficulty arises as to when exactly a difference is first 


\section{Conchusion drazen from the Law}

observed, and why. An appeal to experience seems to show that provided the total change of stimulus is small, there is no observed change in the sense-data, but if it is large the reverse is true. It is hard to see how the latter can be satisfactorily accounted for if the sense-datum itself depends upon nothing but the nature of the stimulus.

Leaving that aside for the moment, however, it should be observed that in any case the assertion of the existence of unperceived differences of sense-data implies that the latter depend only on the nature of their corresponding stimuli, for, if we admit other factors, the effect of these may be such that when two sensedata are not distinguished as different, they are in fact the same. Yet there seems to be no reason why such factors should not actually exist. For example, the nature of a sense-datum might depend partly upon the subject to whom it is presented, or upon the relations existing between its stimulus and the stimuli corresponding to other sense-data presented to the same subject at the same or different times.

For our present purposes it is sufficient to consider the latter possibility alone. Suppose $A, B, C$ are three slightly different stimuli in ascending order of intensity. Experiment shows that the following may happen: If $A$ be applied and then increased to $B$, no difference of sense-data is observed by the subject. If $B$ be now increased to $C$, there is still no difference observed. On the other hand, if $A$ be applied and then increased to $C$, the subject observes a change. Evidently, then, the order of the stimuli enters somehow into the matter, and it is impossible to ascertain without further enquiry 
exactly what part it plays. It may be concluded, as is commonly done, that when the order of stimuli is $A-B-C$, there is actually a difference of sense-data, although it is not observed. But it might equally well be said that the sense-data depend not only on the intensity of the stimuli, but also upon a temporal relation between them, namely the order in which they come. The effect of the latter factor may be such that for the order $A-B-C$ there is no change of sense-data, while for the order $A-C$ there is. We have no grounds for asserting either conclusion dogmatically without going further into the matter.

An objection sometimes urged against the view that sense-data can only be said to have changed when the change is observed, is that it implies that the sensedata vary by leaps and bounds at certain fixed points of transition, so that any pair of stimuli gives rise to distinguishable sense-data, if they lie at opposite sides of a point of transition, however closely they may approach it ${ }^{1}$. This implication is by no means necessary, however. For, in the first place, since the subject must be acted upon by the stimulus (whether fixed or varying) for a finite time, his experience during that time must be considered as a whole and on its own merits. It is thus quite unnecessary to assume certain points of sudden transition; we need only suppose that there are certain points at which a change begins. Moreover, there is no reason to consider these points as fixed. They may quite well depend both on the intensities of the stimuli and on the relations (such as temporal order, for example) between the stimuli ; that is, these points

${ }^{1}$ Stout, op. cit., Bk II, Ch. VII, § 2. 
may in general be different in each individual case. Hence the objection just cited cannot be considered to hold good.

The whole matter is rendered very much clearer if we drop the concept of stimulus and consider the immediate facts. The concept of stimulus is a physical one; that is, it is constructed from the immediate data of sense. Moreover, if we adhere to it, the matter is complicated by the physiological action of the body. If our previous statements are to represent the facts accurately, we must suppose that the final cerebral stimulus which is correlated directly with the sensedatum varies in exact proportion with the physical stimulus.

To fix our ideas, let us suppose that a person $X$, who is blindfolded, is holding a scalepan in which another person $Y$ is silently placing weights. Then the fact of the matter is that certain changes in $Y$ 's sense-data (namely when he adds or takes off weights) are generally correlated with changes in $X$ 's sense-data. This fact is known only by the assertions of $X$. Although this may lead $Y$ to expect that the correlation exists in every case, $X$ 's assertions sometimes contradict this, namely when $Y$ adds a very small weight to a large one.

This statement in terms of the immediate facts includes all the cases mentioned above. In virtue of those facts we are justified in asserting either that there is no change of sense-data when the subject observes none, or that a change may occur unobserved by the subject $^{1}$; but those facts alone afford us no ground for

${ }^{1}$ Of course, one of these two conclusions must be true, but either is in accordance with the facts. 
deciding between the two conclusions. Nor does it help matters to point out that with practice the subject may distinguish differences where before he observed none, unless we make some definite assumption as to the dependence (or otherwise) of sense-data upon the perceiving subject.

Mr Russell states in this connection ${ }^{1}$ that arguments to the effect that differing sense-data must be perceived as different, rest on the assumption that, if $A$ and $B$ are immediate data, and $A$ differs from $B$, then the fact that they differ must also be an immediate datum. We may agree with $\mathrm{Mr}$ Russell that this is a false premise. For knowledge of $A$ and $B$ is knowledge by acquaintance, whereas the knowledge that they differ is "knowledge about," implying a judgment. But this does not affect the point at issue. We are still left with the following dilemma: If no judgment is made, what criterion have we at all by which we may know when sense-data may be said to be different? We must not simply assume that difference of stimulus, or of other people's sense-data, is the criterion, for this begs the whole question. Moreover, we should not know the facts which lead to the formulation of the Weber-Fechner law at all, if the subject did not make judgments on the sense-data, and assert them. On the other hand, if a judgment is made, there still remains the problem as to whether sense-data sometimes differ when judged to be the same, or not. But this is the very question that we are considering. The fact is, that even when the subject is on the look out for a change, he sometimes observes no change when we should expect ${ }^{1}$ Op. cit., Lect. V, p. 144. 
that he would owing to changes in our sense-data of a type generally correlated with changes in his.

The argument for unperceived changes in sensedata is sometimes stated without reference to stimuli in the following way: If a succession of sense-data $A-B-C$ be experienced, it happens on occasions that on referring back in memory from $B$ to $A, C$ to $B$, and $C$ to $A$, no difference can be distinguished between $A$ and $B$, nor between $B$ and $C$, while a difference is distinguished between $A$ and $C$. Hence $A$ and $B$ must really differ, as must also $B$ and $C$, although no difference is perceived.

It is not difficult to discover the fallacy in the above argument. When comparing a present sense-datum $B$ with a past remembered sense-datum $A$, there are two alternative possibilities. Either our judgment of comparison is determined by $B$ and $A$ itself, or by $B$ and some other factor which is largely but not entirely dependent on $A$. Now the first alternative may be ruled out at once, for if it were true it would be equally easy to compare sense-data whatever the time-interval between them-whether it were five seconds or five years. Accordingly we are left with the other alternative, that our comparison of $B$ and $A$ is determined by $B$ and another factor $A_{1}$ (probably in most cases, and perhaps in every case, an after-image or a memoryimage of some kind) largely but not entirely dependent on $A$. Hence in the case considered above, the three judgments of comparison are really determined respectively by the factors $B$ and $A_{1}, C$ and $B_{1}, C$ and $A_{2}-$ where $A_{1}$ is the factor corresponding to $A$ when $B$ is perceived, $B_{1}$ that corresponding to $B$ when $C$ is 
perceived, and $A_{2}$ that corresponding to $A$ when $C$ is perceived. Thus although the judgments were stated in words as $B=A, C=B, C \neq A$, introducing a contradiction, what were really judged may be symbolized as $B=A_{1}, C=B_{1}, C \neq A_{2}$, in which there is no contradiction. This result would be compatible either with likeness or with difference in $A, B, C$, so that the argument we have been considering cannot decide the point at issue.

We can only come to the conclusion, then, that the inference commonly drawn from the Weber-Fechner law, as applied to small differences, is not proven by the facts on which that law is based. It is consistent with those facts, but so also is the opposite hypothesis that sense-data can only be said to differ when they are observed to be different. We are therefore in a position to press the enquiry further by reconsidering the whole matter from a fresh point of view. Without such further enquiry, it is impossible to decide as to which hypothesis is the true one.

\section{The Nature of Sense-Experience}

Experience consists essentially in the presentation of an object to a subject. We may symbolize it as $S p O^{1}$. The fundamental fact from which we start must not be a dualism of "mind" and "matter" such as was conceived by Descartes, for "mind" and "matter" are both secondary conceptions, but this unity of individual experience comprising the duality of subject and object standing in the relation of presentation $p$. Of the terms

\footnotetext{
${ }^{1}$ Cf. James Ward, Psychological Principles, p. 37 I.
} 
thuselated, doubt is sometimes cast upon the existence of $S$ With this point the writer has dealt elsewhere. Sufce it to say that doubt of the existence of the subject is whout significance. In any case, however, since our roblem deals with differences in sense-data, and sinc in perceptual experience $O$ is composed of sensedata the question hinges on the nature of $O$. In partular, we must enquire whether $O$ is conditioned in any ay by the fact that it stands in the relation $p$ to $S$, and, $f$ so, in what way.

he nature of the problem underlying the difficulty raise by the Weber-Fechner law now begins to be apprent. It is, in fact, no less than the crux of the issucbetween realism and spiritualism. The realists conted that $O$ exists quite independently of $S$, and that ts nature is not conditioned in any way by the fact tat it stands in the relation $p$ to $S$. Its entry into that elation is, so to speak, "accidental." It exists indecndently of that relation, and, upon entering into it, th characteristics it already possesses are not altered in an way ${ }^{2}$. On this view there is evidently no difficulty, at an rate prima facie, in supposing that differences of sese-data exist even though unobserved by the subjct. For the spiritualist, on the other hand, the esse $\left(\right.$ the object of experience is necessarily percip $i^{3}$. For im there cannot be a thing of such a nature as $O$, whic is yet not perceived by anybody. Hence, if

${ }^{1} 1$ "Scientific Method in Philosophy and the Foundations of Plurain," Section IV.

${ }^{2}$ se, e.g., R. B. Perry in Present Philosophical Tendencies, Ch. XIII.

${ }^{3}$ J is not, of course, necessary for spiritualism to assume that the being $~ O$ depends only on its being perceived, but merely that the latter one necessary condition of its being. 
spiritualism is to be thoroughgoing, it cannot countenance the assertion that there may be differences of sense-data which are unperceived.

The realist conception of experience is based on the theory of the externality of relations. According to that theory, the being of an object is quite independent of any relations in which it may happen to stand to other objects. Although a relation may add something to the nature of its terms, the characteristics already possessed by the latter are entirely unaltered by it $^{1}$. Hence the being of $O$ is regarded as quite independent of the fact that it stands in the relation $p$ to $S$. The only addition which this relation brings is the awareness of the subject.

In examining this view it is important in the first place to recognize the difference between relations distinguished within the object of experience, that is, relations between the sense-data composing the latter, and the relation of presentation in which that object stands as a whole to the subject. The relation $p$ is evidently unique ${ }^{2}$. One consideration alone makes this sufficiently clear, namely the fact that $S$ cannot be

${ }^{1}$ See, e.g., R. B. Perry, op. cit., Ch. XIII, p. 319 .

2 This is a very important point. For example, R. B. Perry (in his essay in The Nerw Realism) enumerates types of dependence, of which the presentational relation is not one, and then has no diffculty in showing that the latter is not included under any of the types enumerated. Obviously not, since it was excluded by definition, for, owing to its uniqueness, it cannot be subsumed under any other type of relation. All Mr Perry's types refer to relations between objects, whereas we have here a relation between an object and a subject. Mr Perry's case could only hold if he gave a general criterion which all relations of dependence must satisfy, and then showed that the presentational relation did not satisfy it. 
considered to exist out of the relation $p$. For there could not be a subject to whom nothing was presented, in other words, a subject without experience. Hence the existence of $S$ is conditioned thus far by its standing as one term in the relation $p$. This fact gives us sufficient warrant for refusing to assume, without the most searching enquiry, that the being of $O$ is not conditioned in any way by the relation $p$. Seeing that such an entity as a subject of experience to whom nothing is presented cannot exist, it is conceivable, to say the least, that such an entity as an object of experience that is presented to nobody cannot exist either. This is not meant to suggest, of course, that it is impossible for anything to exist which neither knows nor is known, but that if such things should exist, they cannot be considered to be in any way akin either to subjects or to sense-data.

Mr Russell states that the existence of a sense-datum cannot be logically dependent upon that of the subject, for the only way in which the existence of $A$ can be logically dependent upon the existence of $B$, is when $B$ is part of $A^{1}$. But the matter does not appear to be quite so simple in the unique case of subject and object standing in the presentational relation. For let us denote the individual experience $S p O$ by $E$. Supposing it turns out, as we have suggested, that such an entity as $O$ can by its very nature only exist as part of an experience. In that case $E$ would be logically dependent upon $O$, for $O$ is a part of $E$, but the existence of $O$ would also be logically dependent upon

' In "The Relation of Sense-data to Physics," Section III, which originally appeared in No. 4 of the 19r4 volume of Scientia. 
the existence of $E$. Moreover, as we have seen, the existence of $S$ is logically dependent on that of $E$ and vice versa. In such a case then, it would surely not be straining the point to say that the existence of $O$ is logically dependent upon that of $S$.

Leaving aside, however, the question of logical dependence, there is yet another difficulty to face if we refuse to admit that the existence of $O$ is conditioned in any way by the relation $p$. For if we apply the Weber-Fechner law to the limiting case of sense-data of very small intensity, we find that a finite stimulus is needed before any corresponding sense-datum is perceived at all. From this, as we have seen, it would be commonly concluded that the sense-datum does exist, although unperceived. In other words, we should have to grant that there must be parts of $O$ which are not perceived at all. But if this is so, what exactly is the difference between such parts of $O$ and what Mr Russell calls "sensibilia" (that is, entities similar to sensedata in every way except that they do not necessarily form part of an object of experience), and why are we justified in including the ones within $O$ while excluding the others ${ }^{2}$ from $O$ ? And if we are not justified, how can we escape the conclusion that the being of $O$ must depend in part upon its being perceived?

Has the phrase "an unperceived sense-datum" any real meaning? By a sense-datum we must obviously mean something that is "given," and "given" in this sense means "presented to a subject." Can we significantly say that an object is presented yet not perceived?

${ }^{1}$ Op. cit., Section III.

2 I.e., "sensibilia" not forming a part of an object of experience. 
It seems doubtful whether we can, when we take into account the peculiar nature of presentation, but the point need not be insisted on. It is noteworthy, however, that Mr Russell asserts that sense-data "probably never persist unchanged after ceasing to be data." Yet he maintains the existence of unperceived sensibilia which " resemble" sense-data in every way except that they are not perceived. But if two things resemble one another, we must be able to state that they possess a common characteristic. Now what is this characteristic common to sense-data and unperceived sensibilia alike? Is such a thing possible at all ? For the only characteristic common to all sense-data is that of being presented to a subject, and this is here ruled out.

Other considerations make it evident that the object of experience depends in part upon the subject. In the first place, the order of our sense-data is to a certain extent determined by our own activity manifested in movements. To this it would probably be replied that we have only the right to assert that certain motor presentations are followed by changes in sensory presentations. This is true, but a motor presentation follows the idea of the movement, and the latter is consequent only upon the active attention of the subject. Psychological analysis thus reveals that the order of sense-data is determined in part by the activity of the subject. Yet this is not all. Not only the order, but also the nature of the sense-data which make up the object depends partly on the activity of the subject. The growth of perceptual experience consists essentially in an ever-increasing differentiation of the object by

${ }^{1}$ Op. cit., Section IV.

R. S. P. 
the subject ${ }^{1}$. The realists would contend that these differentiations were present in the object all the time, but were only gradually observed by the subject. This statement really brings us to the heart of the whole matter. For the realists, if they are to maintain their view, must evidently look upon the object as made up of a number of mutually external though related units termed sense-data. But this is only a necessary and very close approximation to the facts. The object of experience is actually an indivisible whole, albeit differentiated ${ }^{2}$.

If the realist contention were true, that at a low level of experience the object is characterized by the fact that it comprises many elements not as yet observed, our experience at such a level would be full of "gaps," as it were. This, however, is not the case. The difference between a high and a low level of perceptual experience is simply the difference between a more or a less differentiated unity. But in each case this unity is a given indivisible whole. In each case the subject perceives the whole object, and the latter is only what is perceived by the subject. The fact that the differentiation is much greater in the one case than in the other is due in large measure to the activity of the subject; due also, as we shall see, to other factors; but these other factors are not sense-data which form part of the object from the beginning though unperceived by the subject.

1 Hence the so-called "subjectivity" of the object is not merely physiological as Mr Russell maintains. ["The Relation of Sense-data to Physics," Section III.]

${ }^{2}$ See the essays on "Scientific Method in Philosophy, etc.," Section VI, and "Immortality," Section IV. 
Although the object is determined by the subject to the extent we have seen, it is by no means entirely so determined. Evidently the nature of our sense-data is largely independent of us. In fact we may say roughly that whereas our activity plays a large part in determining the form of our experience, the matter of that experience is independent of us to a very great extent. But although its nature ${ }^{1}$ may be thus independent, it does not follow that its being is also independent. There still remains the question as to the most satisfactory interpretation of the facts. This question we shall now consider.

The realist speaks of the "appearances" presented by "things," but on his theory the appearances are the ultimate entities, and the things are simply logical constructions. On grounds of continuity and resemblance we correlate certain aspects perceived by ourselves and others as being appearances of one thing. The "thing" is simply the class of these correlated aspects ${ }^{2}$. We may grant this view so far as the term "thing" refers to such common-sense objects as tables, chairs, etc.; but there are difficulties in the way of accepting the view that the appearances or aspects are ultimate entities and independent of their presentation to a subject. Mr Russell states that continuity makes it not unreasonable to suppose that things present some appearance at places where there is no percipient subject ${ }^{3}$. But it

1 This word of course implies "form," but the distinction between "matter" and "form" is not meant to be considered absolute here. The distinction between "nature" and "being" is in this case not very clear for reasons which will be fully considered later.

B. Russell, "The Relation of Sense-data to Physics," Section V. ${ }^{3}$ Op. cit., Section III. 
is difficult to see how an appearance can be presented to nobody, unless "appearance" is defined in some very unusual way. Mr Russell's definition does not avoid the difficulty. Such correlated sensibilia as we have mentioned, he defines as "appearances of one thing." Such a definition evidently begs the whole question of the nature of sense-data and their dependence on a percipient. Moreover the difficulties become still more insistent if we try to deal with the question of change. If we define the thing as the class of all its appearances, the thing will change if one of its appearances changes; for instance, if I close my eyes when I am looking at it. But in this case the change is in my eyes, and the realist would maintain that there still existed appearances of the thing nearer to it than my eyes, which were unchanged. Mr Russell deals with the point as follows: "We may say as a matter of definition, that a thing changes when however near to the thing an appearance of it may be, there are changes in appearances as near as, or still nearer to, the thing. On the other hand we shall say that the change is in some other thing if all appearances of the thing which are at not more than a certain distance from the thing remain unchanged, while only comparatively distant appearances of the thing are altered ${ }^{1}$." Now throughout this statement the same "thing" is referred to. But how exactly is it defined? Not as the class of all its appearances, for in that case it would not remain unchanged in the second instance given by $\mathrm{Mr}$ Russell. It would therefore have to be defined as the class of all its appearances at not more than a certain distance from it. But we

1 op. cit., end of Section VIII. 
have still to say what this distance is. Evidently in the case mentioned it would have to be less than the distance of the observer. Hence we cannot define the class of appearances which is to be considered to constitute one thing, without reference to a percipient subject, if any percipient subjects exist. The case is evidently still more complicated when there is a number of percipient subjects at varying distances from the thing.

Supposing, however, while retaining the realist's terminology, we reverse his hypothesis by regarding the object of experience as the appearance to the subject of entities other than the subject. These entities should be by no means identified with the objects of common sense. The latter may still be regarded as logical constructions of sense-data actually perceived by different people at different times and at different places. Passing over for a moment the actual nature of the entities which appear, let us consider what the term "appearance" really means.

It should first of all be noted that an existent being cannot be an immediate datum ${ }^{1}$. It is impossible for anybody other than such a being to know it in its actuality and completeness. If it is not another being, that is, if it is the knowing subject himself, then it evidently cannot be an object of knowledge for that subject. In this case the fact can only be termed "realization." Returning, however, to the case of the relation between the subject and other beings, we may proceed somewhat as follows: "Given a subject $S$ and certain other entities, under appropriate conditions, of which the ex-

${ }^{1} \mathrm{Cf}$, the previous essay, Section II, and the essay on "The Relation of Mind and Body," end of Section II. 
istence of these other entities is the most necessary and important, $S$ will perceive an object $O$ which may be defined as the 'appearance ' of the other entities to $S$." It is obvious that appearance in this sense implies the existence of at least two entities. It must be the appearance of something to something else. The appearance of $A$ to $B$ is neither $A$ nor $B$, but it has no being apart from the existence of $A$ and $B$. Moreover, it is possible that $A$ may change and yet appear the same to $B$. If it appears the same, the appearance is the same; that is, if the object of experience is perceived as the same, it is the same. In this way, the philosophical questions raised by the Weber-Fechner law may be answered. In the first place, there is no thing similar in nature to an object of experience which is not perceived by any subject, although the being of an object of experience is not entirely dependent on the existence of the percipient subject. And in the second place, if the object of experience is perceived by the subject as unchanged, it is unchanged.

It must not be supposed that the term "appearance" is in any way connected with "illusion." "Illusion" is only a significant term when a judgment has been passed on the appearance. Appearances, being immediate data, cannot strictly be said to exist, as we have seen. In fact, such words as "existence," "real," and "unreal," cannot be significantly applied to them at all. Yet they have some sort of being. We can say of them that they are there, and that ends the matter.

It is important to notice that Dr Whitehead and $\mathrm{Mr}$ Russell ${ }^{1}$ have been led from logical considerations to the same conclusions as those just stated with regard I Principia Mathematica, Vol. I, I4. 
to the applicability of such words as "existence" to immediate data. Yet Mr Russell says that we may legitimately enquire as to the existence or reality of other sensibilia inferred from such data ${ }^{1}$. But according to him, sensibilia which are unperceived are essentially similar to sense-data, differing only in the fact of not being perceived. It is difficult to see how terms which are quite insignificant in application to an entity standing in a certain relation (the terms having no reference to that relation), may yet be significant when it does not happen to stand in that relation; and this more especially if we should by any chance adopt Mr Russell's view of the externality of relations ${ }^{2}$.

There remains for philosophy the further question as to the probable nature of the entities which appear. We are not here concerned with a detailed attempt to answer that question, but the possibilities may be briefly indicated and weighed. There are four such possibilities. The entities may be wholly material, or wholly spiritual; they may be partly material and partly spiritual, or they may be neither.

If by "material" we mean to indicate the molecules, atoms, and electrons of physics, it may be said at once that we have no reason whatever to suppose that they

1 In "The Relation of Sense-data to Physics," Section XII.

"It is true that in the second passage quoted, Mr Russell qualifies his assertion by saying that " $x$ is my present sense-datum" and "my present sense-datum exists" are both significant, though " $x$ exists" is not. Clearly "existence" is here used in a special technical sense. But the point is that that very immediacy, which renders such a proposition as " $x$ exists" insignificant, is so essential a characteristic of sense-data, that apart from it they would not be sense-data at all, nor in any way akin to sense-data. 
actually exist. They may all be exhibited as logical functions of sense-data ${ }^{1}$, and any attempt to assign to them in addition a concrete existence is both gratuitous and unnecessary.

With regard to the possibility that the beings which appear to us are neither material nor spiritual, it need only be remarked that such a supposition should be avoided if we can do without it, in accordance with the principle of Occam's razor.

We are now left only with the spiritualistic hypothesis, namely that the beings whose appearance we perceive are other subjects of experience. This hypothesis constitutes pluralism. Certainly some subjects exist. Strictly speaking each of us knows that one subject exists; but, to avoid a barren solipsism, we may make the further assumption of the existence of other people, in common with most schools of thought, including realism.

Selves or subjects of experience cannot be resolved into sense-data, therefore let us suppose that our sensedata are the appearance of other selves to us, thus securing a philosophical economy. On this hypothesis, then, the object of experience is the appearance to the subject of other subjects. The individual experience consists in the interaction of the subject with other subjects, manifested in the increasing differentiation of the object. The object of experience is a presented whole, one and indivisible. The other subjects are many, but their appearance to a given subject is one. As it is not intended, however, to indicate here any-

${ }^{1}$ See, e.g., B. Russell's Our Knowledge of the External World, Lectures III and IV. 
thing more than the relative probabilities of the possible hypotheses to which our interpretation of the WeberFechner law gives rise, we may now conclude by summarizing the results already arrived at.

\section{Summary and Conclusion}

Our enquiry commenced with an examination of certain conclusions commonly drawn from the experimental facts on which the Weber-Fechner law is based, and of the reasons generally assigned for those conclusions. The latter assert that unobserved differences of sense-data must sometimes exist even when the subject is on the lookout for such differences. A particular case of great importance occurs in connection with sense-data of a comparatively low degree of intensity. In such cases it is asserted that sense-data must sometimes exist which are not perceived at all.

Analysis showed that these assertions must be considered as not proven by the experimental facts to which appeal is made. For no allowance is granted for the possibility of the existence of other factors than those generally taken account of in determining the result. Such possible factors, for example, are the particular temporal order of the stimuli, and the peculiar nature of the presentational relation of sense-data to the subject. The latter of these appeared in the sequel as by far the more important. In any case, however, should such factors be effective, their action may be in the nature of a compensation for the effects of the other factors. Taking account of this possibility, it was seen that the experimental facts, which are generally con- 
sidered with reference to the latter factors alone, while compatible with the conclusions commonly drawn, are equally compatible with the opposite conclusions. In accordance with the latter it would be asserted that no objects of a nature essentially akin to sense-data can exist unperceived, and that sense-data which are perceived as the same, are the same. Hence it became evident that further investigation was necessary before a definite statement on the matter could be made.

In any philosophical enquiry of this nature, we must start, not from a dualism of mind and matter, but from the unity of individual experience comprising the duality of subject and object standing in a relation of presentation. The effects of the traditional dualism of matter and mind still linger in philosophical thought, and are manifested, for example, in the vague use of such terms as " mental." This word is used in at least two distinct ways. In the first place, it is used of such things as dreams or hallucinations which are supposed to exist only " in the mind" of the subject, whatever that may mean. The only way in which we could modify this usage so as to give a precise meaning to the term "mental," would be by defining it as applying to those portions of the object of experience which only exist in so far as they are perceived by the subject. With this meaning of the term not only dreams and similar things but also ordinary sense-data might be mental. Such a use of the word is, however, highly unsatisfactory.

In the second place the term "mental" is sometimes supposed to refer to anything which is part of the subject-as if a subject could have "parts." Probably what are here indicated, are such things as thinking, willing, 
and desiring. These are essentially connected with the feeling and activity of the subject, and it is here that we have the strictly correct use of the word. "Mental" should only be used to characterize the feeling and activity of the subject.

Mr Russell supposes that sense-data are not mental but physical, that is, part of the subject-matter of physics ${ }^{1}$. Granted this, he appears to assume that it follows that the being of sense-data is not dependent in any way upon their being perceived. Such a conclusion, however, is by no means necessary. We may grant that sense-data are not mental according to our definition of the term, and also that ultimately physics deals with sense-data. But this does not shed any light on the question of their dependence upon perception. It is sometimes urged in support of the view that they are independent of perception, that physics can describe the object of experience and make verifiable predictions about it without any reference to the subject or to perception. But even if we grant this, it is quite irrelevant. One example is sufficient to show that this is the case. I can observe the positions and movements of the hands of my watch, and make true predictions as to their future positions, without any reference whatever to the mainspring. Yet the latter is the sine qua non of all that I have observed and inferred. It is evident that we continually describe things and make successful inferences about them, without any reference to the necessary conditions of their existence.

To solve our problem we must endeavour to determine what is the real nature of the object of experience,

1 "The Relation of Sense-data to Physics," Section III. 
or, if we cannot obtain a definitive solution, to determine the hypothesis which fits the facts most satisfactorily. The real issue at stake is that between realism and spiritualism. If sense-data can exist unperceived, there is no reason why objects essentially akin to sense-data should not exist yet without forming part of an individual experience at all. In such a case the theory that reality is wholly spiritual goes by the board. On the other hand, if it turns out that objects such as sense-data are essentially characterized by presentation to a subject, realism, at all events in its modern form, cannot be true.

Our first step in the analysis of the question was to comment on the theory of the externality of relations by calling attention to the importance of making the necessary distinction between relations holding within the object of experience, and that relation of which the latter is one of the terms, namely the presentational relation. The presentational relation is evidently unique. There cannot be a subject to whom nothing is presented; that is, the subject is essentially characterized by always standing as one term of the relation $p$. Evidently, then, it is at least possible that the object may be essentially characterized by always standing as the other term of the relation $p$. The point demands further investigation.

A realism of the type maintained by Mr Russell is faced with a dilemma. For if we accept the conclusion commonly drawn from the Weber-Fechner law it follows that there must be three classes of sensibilia. Those which do not form part of an object of experience; those which do, but are not perceived; and those which do, 
and are perceived. What, then, is the criterion of difference between members of the first two classes? What difference can there be? And if we admit that there $i s$ no difference, it follows that all sensibilia which form part of an object of experience must be perceived. On the other hand, if we disagree with the common conclusion drawn from the Weber-Fechner law, the same result follows at once. If we accept this result, we are under no necessity of asserting the existence of particulars essentially akin to sense-data, which yet form no part of an object of experience, nor is their existence even rendered probable.

A similar dilemma arises in the case of small differences in sense-data. For the only possible criterion by which we can certainly decide whether two sense-data differ or not, is the judgment of the percipient subject. According to this we should have to say that if two sense-data appear the same to the subject, they are the same. On the other hand, if no judgment is made, not only have we no means of deciding whether two sensedata perceived by the given subject differ or not, but we can obtain no information whatever about the sensedata.

Evidently the object of experience is partly dependent upon the subject. The movements of attention and, in fact, the whole growth of experience exemplify this. On the other hand, it is no less evident (provided we reject solipsism) that the object is not wholly dependent on the subject. The activity of the latter plays a part which is largely formative. The further back we go in experience, the cruder does the object appear. Yet it is never entirely formless. We may therefore 
conclude that whereas the form of the object of experience is largely determined by the shaping activity of the subject, the matter thereof is no less determined by entities other than the subject. There still remains, however, the question as to the conditions upon which the being of the object depends.

As we have seen, the view that particulars essentially resembling objects of experience may exist without forming part of an experience is beset with great difficulties. We therefore attempted to frame a more satisfactory hypothesis. Let us suppose that two entities $A$ and $B$ exist, $A$ being a percipient subject. Then given certain conditions, of which the existence of $B$ is the most necessary and important, $A$ perceives a certain object. Evidently this object is not $A$. But neither is it $B$, seeing that an existent thing cannot be an immediate datum of perception, for clearly the thing in its actual essence cannot be an object of acquaintance to anything else. Hence we defined the object which $A$ perceives as the "appearance" of $B$ to $A$. For the reasons stated, it does not exist in the sense in which $A$ and $B$ exist. Yet it has being in the sense that it is there. On the other hand, its being is dependent on the existence of both $A$ and $B$. We may therefore regard the object of experience as the appearance to the subject of entities other than the subject. The entities may be many, but the appearance is an indivisible unity, being presented to one subject.

On this view it would appear that realism and the traditional idealism are half truths. Realism is right in asserting that the being of sense-data is not entirely dependent on their being perceived, but wrong in so 
far as it asserts that they are a type of objects whose being is quite independent of perception. Idealism, on the other hand, is right in maintaining that if sensedata are to be at all, they must be perceived, but wrong in maintaining that their being perceived is the only condition of their being. The reconciliation is effected by regarding both the existence of a percipient subject and also the existence of some other entity or entities as necessary conditions of the being of sense-data. The latter are the appearance of something to something else.

There remains for the philosopher the problem of the nature of these entities which appear to the subject. In selecting a working hypothesis we should be guided by two principles. In the first place the introduction of unnecessary entities whose real nature remains a mystery should be avoided; and in the second place we should choose if possible beings whose nature we can satisfactorily realize. In accordance with the first principle we may rule out objects of common sense such as chairs and tables, for these are capable of being exhibited as functions of sense-data, and can lay no claim to concrete existence as objects apart from, and in addition to, sense-data. On the same count, physical entities such as atoms, molecules, electrons, etc., may also be ruled out. Physics certainly carries its constructions one step further than ordinary common sense, but many of the objects with which it deals have already been shown to be simply logical functions of sense-data, and analogy leaves little doubt that all physical objects are of a similar nature.

The second principle evidently points the way to 
the hypothesis of pluralistic spiritualism. According to that hypothesis the beings which appear to the subject are other subjects differing from himself only in degree of mental development. The individual experience thus consists in the interaction of the subject with other subjects. The object of experience is the appearance to him of the other subjects, and his interaction with them is manifested in the ever-increasing differentiation of the object. With the development of this hypothesis, the present writer has dealt elsewhere ${ }^{1}$. It is sufficient here to indicate it as a possibility, and to point out the facts we have mentioned as appearing to favour its inception.

We have seen then, that an examination of the facts on which the Weber-Fechner law is based, and of the general nature of individual sense experience, leads to the following conclusions: Firstly, the reasons generally asserted for supposing that sense-data sometimes differ when perceived as the same, and that there must be sense-data which are not perceived at all, are not conclusive; in fact they are quite compatible with the opposite view. Secondly, there cannot be such things as unperceived sense-data nor objects essentially resembling sense-data and yet not forming a part of any object of experience; moreover, sense-data perceived as the same must actually be the same according to any applicable criterion and in any sense in which the word "same" is significant of sense-data. Thirdly, the difficulties can be resolved most satisfactorily if sense-data are considered to be the appearance to the subject of

${ }^{1}$ In "Scientific Method in Philosophy and the Foundations of Pluralism." 
entities other than the subject. Finally, while the facts we have had under review point to no absolutely definite conclusion as to the nature of these entities, certain indications contained in those facts, together with the principle of philosophical economy, make it probable that the most satisfactory hypothesis is to be found in the assertion that those beings which appear to the subject are also subjects of experience, differing only in degree of mentality. Such an hypothesis is in conformity with the views of modern pluralists, and can be shown to be capable of providing a satisfactory solution of many of the traditional problems with which philosophy is called upon to deal. 


\section{THE NOTION OF A DETERMINISTIC SYSTEM}

\section{IntRODUCTION}

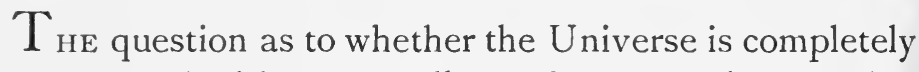
determined, either eternally or for a very long period of time, by unchanging laws, has provided abundant material for discussion to thinkers of all ages. The prominence given to it in the great speculative systems of the past is attributable to the fact that it has for every man a deep personal interest. Physical science has been largely successful in reducing the world we perceive to a system governed by fixed laws on the basis of which we are enabled to make successful calculations as to the state of the material universe past, present, and future. In face of this reduction to a calculable order, we are compelled to reckon with the possibility that those things with which physics has not hitherto dealt may themselves be reducible to an orderly system concerning which confident predictions based on calculation are, at any rate theoretically, possible. Yet it has always come hard to men to admit that the subjective impulses to which human actions owe their inception, are objectively determined in such a way that an observer provided with sufficient data could foretell the future behaviour of any living being. The reluctance to make such an admission is by no means unjustified, for the question is a very subtle one, and, in spite of endless 
argument, it can hardly be said that a definite and satisfactory solution has ever yet been put forward.

Verbal ambiguity is to be found at the root of very many of the difficulties which have arisen in connection with the problem of determinism. It will therefore be necessary to make quite clear what is meant by a "deterministic system," by rendering precise the notion which is at the back of men's minds when they speak of things as being eternally "predestined" or "determined." Words and phrases such as these are used in various meanings which tend to become inextricably confused with one another. In the first place there is the idea of physical compulsion. It is suggested that the behaviour, not only of the inorganic world, but also of the individuals composing the organic world, is the necessary and inevitable outcome of an endless chain of purely physical causation rigidly fixed by a number of unalterable laws. In other words, the mental impulses from which our actions originate are held by this view to be entirely due to conditions which are purely material. Now it is evident that our actions are not unrestricted physically. They must be adapted to our material environment, and are therefore governed, at least in part, by the laws which hold in that environment. Yet it is no less evident that the mind which originates these actions, and which in fact takes account of the limitations imposed by matter, is not itself necessarily determined by matter. Consequently determinism in this sense of physical compulsion is now accepted by very few people. Whether there is logical interdependence of mind and matter is, of course, an entirely different question. 


\section{6 The Notion of a Deterministic System}

There is another sense in which everything is sometimes said to be determined, namely that it is what it is. The past has been what it has been, the present is what it is, the future will be what it will be-all are by this fact equally determined. Closer examination of such statements as these, however, seems to indicate that all they assert is that everything has a "what," i.e., a definite nature. This is quite true. Since the time of Hegel, at any rate, it has been recognized for the most part that Pure Being is identical with Non-Being. All Being is determinate. But all this sheds no light whatever on such questions as, for example, that of free-will. A volition is what it is, but it may be free. Moreover, the statement that the future will be what it will be, is quite irrelevant to the question as to whether the future is determined now. All it asserts is that the future, when it comes, will be determinate, and this is very different from asserting that it is in fact determined at the present time. Evidently the distinction should be made clear between the meanings of the words "determinate" and "determined." In any case, however, a "deterministic system" is not generally taken to mean simply a system in which everything is what it is.

Although we may know, then, that the state of a system at any time is determinate, this does not give us sufficient ground for describing the system as "deterministic" in the ordinary sense of that word. The additional condition is required that the state of the system at any time, past, present, or future, within a given interval, should be susceptible of calculation under suitable conditions. We thus arrive at the notion 
of a system the course of which during any given interval is subject to perfectly definite laws capable of precise statement. If these laws are to be precise enough to render possible calculation and prediction to a given order of approximation, they must evidently be such that they can be enunciated in quantitative terms. If they are known, and if there are also certain data describing the state of the system at given times, a functional relation may be obtained whereby we can determine at once the state of the system at any time within the interval considered, by assigning appropriate values to the variables in the functional relation. The process thus consists in combining the laws to which the state of the system must at all times conform, with certain data, and hence obtaining a functional relation which is equivalent to a propositional function describing the "generalized" state of the system, as it were. By assigning suitable values to the arguments in the propositional function, it is transformed into a proposition descriptive of the state of the system at a particular time.

A deterministic system is therefore one the state of which at any time is theoretically calculable given its states at certain definite times. In such a system the future, no less than the past, is irrevocably determined. Moreover, it should be noticed that it is unnecessary for the purpose of defining a deterministic system conformably with what appears to be the essence of the vague notions generally formed of such a system, to go into any discussion of "interaction" or "causation" within the system. Whether any processes corresponding to such terms exist or not, is irrelevant to the question. 


\section{8 The Notion of a Deterministic System}

Whatever the inner workings of the system, if it be in fact amenable to calculation and prediction in the manner described, it thereby satisfies any condition that might be laid down by even the most rigid determinist.

In the definition given, the state of the system was considered as "theoretically" calculable. The number and complexity of the laws and other data might be so great (perhaps infinite) that the necessary computation could not be carried out in practice, at any rate by human beings. In spite of this, however, it is evident that the system is yet deterministic, provided the necessary data do not comprise all actual states of the system. If the latter were the case, there would no longer be any other assignable values of the arguments in the propositional function. In other words, this function would lapse, with the disappearance of the variables, into a proposition consisting merely in a theoretical description of the whole history of the system in the interval considered. If, however, the necessary data are not of this order of complexity, the system is deterministic, although exact calculation of its states might not be capable of practical accomplishment.

The present state of the problem of determinism is characterized chiefly by the fact that only recently has a stage been reached in which the problem itself, quite apart from attempted solutions of it, is set forth without ambiguity. It is recognized that in deciding whether a system is deterministic or not it is quite unnecessary to know what may be called the "inner mechanism" of the system. Empirical observation alone is sufficient to decide whether the behaviour of the system, no 
matter from what it originates, is such as to render successful calculated predictions of its future course based on general laws derived from that observation. It is, of course, an assumption that the system will continue to follow the same laws in the future, but, granted that assumption on the grounds of probability, it follows that the fate of the system is rigidly determined.

For philosophy there arises the problem as to whether the universe is a deterministic system in the sense we have defined. If it should turn out that any parts of the universe are free, the universe as a whole cannot be a deterministic system, for parts which might be in themselves determined are liable to interference from those which are free. It may of course happen that some regions of the universe are so remote from the vicinities in which freedom exists that they are practically unaffected by it. Such regions, considered in isolation, would approximate very closely to deterministic systems, although the universe as a whole would not be deterministic.

It is evident that it is important to make clear the meaning of the term "free." This task, however, will be postponed for later consideration. Meanwhile we may proceed to consider a statement of the case for determinism, which puts the whole matter very clearly, and which suggests very forcibly the arguments in favour of a deterministic view of the universe.

\section{The Case for Determinism}

The account just referred to of a deterministic system is due to $\mathrm{Mr}$ Bertrand Russell, and to it all 


\section{I20 The Notion of a Deterministic System}

the foregoing has been intended tolead up ${ }^{1}$. Mr Russell's definition is as follows: "A system is said to be 'deterministic' when, given certain data, $e_{1}, e_{2}, \ldots e_{n}$, at times $t_{1}, t_{2}, \ldots t_{n}$ respectively, concerning this system, if $E_{t}$ is the state of the system at any time $t$, there is a functional relation of the form

$$
E_{t}=f\left(e_{1}, t_{1}, e_{2}, t_{2}, \ldots \ldots, e_{n}, t_{n}, t\right) .
$$

The system will be deterministic throughout a given period if $t$, in the above formula, may be any time within that period, though outside that period the formula may be no longer true." In this definition, all unnecessary lumber, such as the notion of causation, is cleared away. As Mr Russell points out, the common view is that inference of the future from the past is made possible by the principle of causality. But the explicit introduction of that principle, bringing, as it does, in its train all sorts of problems as to the exact nature of causality, is superfluous. Grantedour functional relation, obtained by empirical observation, any further postulate as to the inner working of the system is irrelevant in deciding whether the system is deterministic or not. The very fact of the existence of the functional relation is sufficient to establish the determinism of the

1 In all that follows, the reference is to an essay on "The Notion of Cause," by B. Russell, which originally appeared in the Proceedings of the Aristotelian Society for 1912-13. It was then published in Scientia, Vol. 1913, N. xxix-3. Recently it has appeared in a volume entitled Mysticism and Logic and other Essays. The references are to pp. $199 \mathrm{ff}$. of the latter, or to $\mathrm{pp}$. $33 \mathrm{Iff}$. of the number of Scientia mentioned. It should be said at once that Mr Russell does not himself come to a definite conclusion here as to whether the Universe is deterministic or not, though he inclines to the former view. 
system. Mr Russell calls the data $e_{1}, e_{2}, \ldots e_{n}$ "determinants" of the system, remarking that it is evident that a system having one set of determinants will in general have many. In view of the fact that his account of a deterministic system is perfectly clear and unambiguous, and comprises all that is generally contained in the notion of such a system, we shall take it as a basis for criticism and discussion.

The fact that the future will be what it will be is regarded by $\mathrm{Mr}$ Russell as being of considerable importance. For reasons already given we do not take this view. It is true, as Mr Russell says, that we cannot make the future other than it will be, but this is very different from saying that the future is in fact determined now. For evidently what the future will be is in part determined by our actions, and we cannot decide as to whether the future is determined now, unless we know whether our actions between the present and any given future date form part of a deterministic system or not. It is true that those actions themselves will be what they will be, but here again the reference is to the future. To say that any future event whatever is determined now by the fact that when it comes it will be determinate, is simply equivalent to saying that everything is something, a true enough statement but hardly to be urged as an argument in favour of however lax a determinism. Mr Russell does not insist on the point, recognizing that it is not what people usually mean by "determinism," but confines his attention for the most part to a deterministic system as defined in the quotation given above.

Two important illustrations are given by $\mathrm{Mr}$ Russell 


\section{I22 The Notion of a Deterministic System}

as bringing out clearly the conception of a deterministic system. Both refer to the possible nature of the universe. In the first the hypothesis of psycho-physical parallelism is introduced-that is, it is assumed that to a given state of brain a given state of mind always corresponds. The highly probable assumption is also made that to a given state of a certain brain a given state of the whole material universe corresponds, since the recurrence of exactly the same brain-state is extremely unlikely. Hence, if $n$ states of the material universe are determinants of the material universe, they would also be determinants of the whole universe, mental and material, as would also the corresponding $n$ states of a given man's mind.

Evidently, if the above holds, the universe, including man, forms a deterministic system, and conversely if the universe is determined it must be in some way which is closely represented by the above. Moreover, it should be noted that psycho-physical parallelism is not an assumption essential to the latter. For psychophysical parallelism is rather a methodological principle than an hypothesis, and the fact of correspondence which it asserts would exist equally if there were any form of interaction between mind and brain. It is true, as Mr Russell remarks, that the correspondence between mind and brain may not be one-one, but many-one, or one-many; but in that case the universe would still be deterministic (though its determinants might be more complex) provided the scope of the correspondence on the multiple side was determined.

The second illustration relates to the dispute between the teleological and the mechanistic views of the world. 
A "mechanical" sytem is reasonably defined as one having a set of deteminants which are purely material, such as the positios of certain pieces of matter at certain times. Butif some account of the universe such as that, for eximple, given in the first illustration were true, all meral facts, including purposes and desires, as well asthe universe of matter, would be determined by suc a set of material determinants. Hence purposes, wether realized or not, could exist in a mechanical syst $\mathrm{m}$, so that the latter might also in that case be fairly esignated "teleological." Thus if the view taken is ccrect, the terms "teleological " and "mechanical" are nt incompatible. There might be a mechanical syster which was also teleological and vice versa.

Clearly these tv: illustrations are particularly valuable, for the first sows us under what form we must conceive the univere if it is actually determined as a whole, while the secnd indicates one important consequence which woulcnecessarily follow in a universe of that nature.

Evidently if we rrm part of such a world we cannot be content to regar ourselves as "free" in any satisfactory sense; for sven (say) the positions of certain pieces of matter at crtain times, all our actions would be theoretically caiulable, past, present, and future. Even now our destry would be irrevocably fixed by the laws of mathemics. Could a sterner necessity, a more unbending tasmaster, be imagined?

Determinism pcsesses as its chief advocate the success of physicalscience. Nobody pretends that our knowledge of the material universe is all-inclusive. 


\section{I24 The Notion of a Deterministic System}

But science has dealt so remarkably with the limited portions in space and time at our disposal, by weeding out, in any given case, the superfluous (because negligibly effectual) accompaniments so as to temper the problem to our intellectual capacity, that we feel little difficulty in thought in extending the process, by analogy, to the performances of a Laplacean calculator who, given certain data, would derive the knowledge of all things. For a mind thus capable of grasping the infinite complexity of the determinants involved, the universe would be an open book. The success of science seems to render it highly probable that the material universe is completely determined by a limited (though perhaps infinite) number of material data, and hence, granted the existence of the psycho-physical correspondence, that the mental universe is also completely determined by those data. If we are not to pass unchallenged a view of such far-reaching significance, it will be necessary to analyze fully the grounds on which it is based. Only thus can we arrive at a true estimate of the credibility to be attached to it.

\section{Analysis of Determinism}

The first step in the analysis of determinism in the sense which has been defined, consists in the examination of the functional relation

$$
E_{t}=f\left(e_{1}, t_{1}, e_{2}, t_{2}, \ldots e_{n}, t_{n}, t\right),
$$

upon the probable existence of which the case for determinism rests. If the state of a system at any particular time is given by such a relation, what exactly is it that thereby determines the system? The deter- 
minants $e_{1}, e_{2}, \ldots e_{n}$ with the corresponding times are not sufficient. From them alone we can derive no information about the system. Given the function, they fix its value for particular values of the variable; that is, they may be considered as necessary and sufficient determinants of the values of the function in particular cases. But for the system to be determined, not only must the data $e_{1}, t_{1}, \ldots e_{n}, t_{n}$ be given, but also the relations between them, that is, the way in which they enter into the function $f$. In other words, the form of that function must be given, and it is therefore a determinant of the system equally with the data $e_{1}, e_{2}$, etc. Summing up then, we must regard $e_{1}, e_{2}, \ldots e_{n}$, together with the form of the function connecting them with one another and with the variable $t$, as the determinants of the system.

Let us now consider the course we should have to pursue, had we the intellectual grasp of the Laplacean calculator, in order to discover from observation whether the universe is a deterministic system or not, i.e., whether there exists for it a functional relation such as $E_{t}=f$. At least one possible type of the necessary determinants $e_{1}, e_{2}, \ldots e_{n}$ would probably be discoverable from empirical inspection alone, as is generally found to be the case in scientific observation. But the verification of this possibility and the number of determinants thus required, together with the form of the function into which they enter as constituents and which is the remaining determinant, would only be certainly demonstrated in that final synthesis whereby the functional relation is constructed in its completeness.

In the first place, we must not assume that the 


\section{I26 The Notion of a Deterministic System}

course of the material world is entirely independent of mind. Prima facie it is not so, and should the appearance be misleading, that fact could only be demonstrated in the course of our calculation. Consequently our first step, after a course of exhaustive experiment and observation of mind and matter and the interaction (real or apparent) between them, would be to collect all the results of that empirical procedure under a number of general laws. This number should be the minimum possible in accordance with the results so far obtained.

The next step would be the inspection of these general laws with a view to reducing their number. If our process had been carried on in a manner more or less analogous to the evolution of science, the laws would have been obtained as a result of many relatively independent lines of enquiry. An hypothesis fitting all the facts must now be sought by means of which the phenomena can be brought under a common heading, and the laws to which they conform synthesized if possible into one all-embracing formula. This is the ideal to which all science turns its endeavour in dealing with the material world. Examples of it on a comparatively small scale are common enough. For instance the kinetic theory of matter enables us to reduce many of the facts observed in the study of Heat and Light to mere manifestations of an under-lying process which is purely mechanical. The science of Heat proceeded originally as an enquiry quite independent of the science of mechanics, yet in the end it has been reduced to a common basis with the latter. The theoretical investigation we are considering would, of course, be far more 
complicated, for in it we are dealing not only with all material phenomena, but with all mental facts as well. But in any case one important point is evident, namely that a necessary condition of the possibility of reducing the laws we have obtained to a single formula, is that those laws should be capable of precise statement, i.e., of being put into exact quantitative form. If this condition is fulfilled, and if we can relate all the quantities to which the different laws refer, the way is clear for the construction, by numerical calculation, of that singlefunctional relation which shall determine the whole universe.

In the course of our calculation it might appear that we should be led to a functional relation capable of statement in different ways according to our choice of the determinants $e_{1}, e_{2}, \ldots e_{n}$. At this stage of the calculation we should have a number of data at our disposal all of which would not be found to be necessary in achieving the final result. Our decision as to which set of independent data to select would then depend on the particular form we wished our function to take in virtue of the nature of its determinants. Possibly we might be able to make the latter all material, or all mental, or partly material and partly mental. If, however, it turned out that by appropriate manipulation we could eliminate all the mental determinants and yet arrive at a single functional relation, the universe would be a deterministic system whose history, including the history of every living being in it, would be fixed by a set of purely material determinants. Given some such data as the positions of certain pieces of matter at certain times, we could predict with absolute certainty the future behaviour of any man. Discredited astro- 


\section{I28 The Notion of a Deterministic System}

logers may perhaps draw some comfort from this consideration!

It follows from the foregoing that if a system is to be deterministic in the sense we have been considering, it must be one of which quantitative notions are significant, that is, one whose state at any time is capable of being described in terms of quantities which are theoretically measurable. For example, qualities, as such, cannot enter as constituents into the functional relation which gives the state of the system, seeing that qualities cannot be exactly specified but only indicated-to be comprehended they must be experienced. Yet it may be possible to indicate the qualities by quantitative concepts, just as, for instance, we correlate "red" with a certain wave-length or range of wave-lengths. If this is so, the quantity may enter into the functional relation, thus "representing," as it were, the quality, and results deduced from the relation will be valid and capable of being re-interpreted, where necessary, in terms of qualities. If, however, it is impossible to make precise quantitative notions in any way significant of the system, the latter cannot be deterministic.

Another possibility suggests itself. There might be a system the state of which at any time is capable of description in terms of measurable quantities, and yet for which no functional relation exists. Mr Russell makes the following statement in this connection: "If formulae of any degree of complexity, however great, are admitted, it would seem that any system, whose state at a given moment is a function of certain measurable quantities, must be a deterministic system. Let us consider, in illustration, a single material particle, 
whose co-ordinates at time $t$ are $x_{t}, y_{t}, z_{t}$. Then, however the particle moves, there must be, theoretically, functions $f_{1}, f_{2}, f_{3}$, such that

$$
x_{t}=f_{1}(t), y_{t}=f_{2}(t), z_{t}=f_{3}(t) . "
$$

But let us take another example. Consider two material particles attracting one another with a force which is some function of the distance between them. Now suppose this function itself varies, also that the law of its variation varies, and so on. If at any stage of this regress (which may be infinite) the law of variation were known, we could construct our functional relation. This, apparently, is what Mr Russell means when he makes the proviso that formulae of any degree of complexity, however great, should be admitted. But it is conceivable that we should never (even after infinite regress) come to a law of variation. The variation might conceivably be purely haphazard, or at least containing a haphazard element which renders any precise statement of a law impossible. Whether such a system could exist is not the question. At any rate we can imagine it to exist. Its state at any time could be exactly described in terms of measurable quantities, such as the co-ordinates of the particles and their velocities and accelerations; but no functional relation could be constructed giving its state at any time ${ }^{1}$. Such

${ }^{1}$ It may be granted that Mr Russell's contention is true if it merely implies that for any moving particle there is a one-many relation between its positions and the instants of time. This assertion, however, is simply equivalent to saying that such-and-such an entity (the particle) exists in a space-time continuum. But the point is that in a case such as that considered, it would be impossible to construct (even theoretically) equations of the type $x_{t}=f_{1}(t)$, etc., from which, given the positions at some (not all) of the instants in the interval considered, we could infer the positions at the other instants. That

R. S. P. 


\section{I30 The Notion of a Deterministic System}

a system would be called "non-deterministic" or, to use Mr Russell's word, "capricious." If, however, it is not even possible to describe the state of the system in quantitative terms, because quantitative notions are not significant of it, then the words "deterministic" and "non-deterministic" are not significant of it either.

Let us now consider what must be the essential characteristics of a system of which quantitative notions are significant, and to which in consequence numerical calculation may be applied-calculation which will be successful, at least within limits, unless the system is wholly "capricious."

Quantity is expressed by means of number. Number is a property of classes. A given number is the common property possessed by all classes having that number of members. Now a class is a collection of objects (using the last term in its widest sense), and the latter may be considered as units. Replacing, permissibly for formal purposes, the common property defining members of a class by that class as a whole, we have as the definition of the number $n$, the class of all classes of $n$ units ${ }^{1}$. This definition in its first intention applies only to positive integers, but the concept can be extended without great difficulty to negative, fractional, and irrational numbers.

The quantities which spring naturally to mind at once are those termed "extensive" quantities, i.e., those having a nature such that a given quantity may be is, no matter how many elements of the motion were known, they could not be made the basis of inference to unknown elements. The inference of the position at any instant would presuppose knowledge of that position, thus reducing the process to a vicious circle.

${ }^{1}$ See, e.g., Dr Whitehead and B. Russell's Principia Mathematica, Vol. I, Part II. 
regarded as the sum of smaller quantities of the same kind, which we may term "parts" of the given quantity. It is common to divide such quantities into two types, viz. those associated with a finite number of discrete objects, and those which cannot ultimately be regarded as made up of a finite number of parts. The former are used in statements such, for example, as that referring to the number of legs of a certain species of insect, or, to give another instance, in dealing with phenomena depending on the number of molecules in a given volume of a gas. In such cases, any one of the discrete parts forms a natural unit of measurement. To the latter type belong such quantities as distances, which cannot be considered to be composed ultimately of a finite number of parts. For our purposes, however, it is not the difference between the two types which is important, but the characteristic which they have in common, namely that any given finite quantity ${ }^{1}$ may be regarded as the sum of a finite number of smaller finite parts, these parts being themselves quantities of the same nature as the given quantity.

In addition to quantities of extensive magnitude, science also has to deal with those having "intensive" magnitude, such as density and temperature. We cannot regard a density as the sum of other densities without great ambiguity. But it is important to notice that, in any case, the measurement of such quantities is only effected by correlating them with quantities possessing extensive magnitude. Thus, if the notions of quantity and calculation are to be significant at all, we must ultimately deal in every case with extensive quantity,

${ }^{1}$ In the case of quantities of the first type, the given quantity must evidently comprise at least two of the discreta. 


\section{I32 The Notion of a Deterministic System}

i.e., with things which may be considered as made up of parts similar in nature to themselves.

Intensive quantities are similar in one respect to certain qualities, such as those of colour, seeing that they may be specified by correlation with extensive quantities. In fact, strictly speaking, the term "quantity" might well be restricted to the latter. For if there were any actual entities corresponding to things such as temperature and density, having what we call "intensive" magnitude, they would be really more akin to abstract qualities or states. Probably the only reason we call them "quantities" at all is by an illegitimate transference of idea, because we can correlate them with true quantities. The last point is made clear by the consideration of objects of a certain kind which are supposed to be intensively quantitative, namely sensedata. For example, people commonly regard a bright yellow light as having in some way a greater magnitude than a dim yellow light. But the difference is purely qualitative. Certainly both lights are yellow, but the difference between bright and dim is qualitative; just as red and yellow are both colours, but the difference between them is entirely qualitative. That the differences of sense-data in respect of intensity are purely qualitative is shown by the fact that there could be no objective standard of measurement for them'. To assign the number Io (say) to a sense-datum of a certain intensity, would be arbitrary and meaningless. Fechner made an ingenious attempt to construct a system based on a "least perceptible" difference of intensity. Not only, however, would this difference vary with different

${ }^{1}$ The nature of the intensity of sense-data is a point of considerable interest, and is dealt with at length in the next essay. 
people and probably also with the same person for different total situations, but it is itself qualitative. It is meaningless to talk of a difference in intensity of sense-data as being so many times the "least perceptible" difference. All such attempts reduce ultimately to correlation with true (i.e., extensive) quantities, viz. the physical stimuli concerned. Moreover, it should be remembered that all physical concepts, whether those such as mass and energy, or those such as density and temperature, are really constructions of sense-data. Hence the possibility of applying quantitative notions to what is perceived, will depend finally on whether the object of experience may be regarded as made up of parts (sense-data) standing in certain relations, or not. To this we shall return shortly.

Quantity, then, is expressed by a number of units, one important condition being that while dealing with a fixed type of quantity the units must be homogeneous. From the above it is evident that quantity is actually significant only of things which can be considered to be made up of parts, these parts constituting the units. Evidently the number expressing a fixed quantity will depend on the scale chosen, that is on the part selected as the unit of measurement.

The foregoing is made clear by considering its application to the world of physics. Evidently the latter is deterministic, if the fundamental postulates of physics be granted. In any case, quantitative notions are significant of it. Let us endeavour to work back to the ultimate reason for this.

The unitary entities constituting the universe as conceived by physical science are points, instants, and particles. Such entities as these are capable of being 


\section{I34 The Notion of a Deterministic System}

exhibited as logical constructions of the immediate data of sense ${ }^{1}$; they are not inferences from the latter. The physical conceptions which are psychologically primitive are those of force, duration, and distance. The notion of mass is derivative. In ordering our ideas, however, it is common to make a re-arrangement by taking the concepts of mass, time (i.e., lapse of time), and length as logically prior, and making that of force derivative. On these fundamental conceptions the science of Mechanics is based, and all Physics is based on Mechanics. It is true that as physics has developed it has been found necessary to introduce two more fundamental quantities, namely (as the most convenient choice) temperature, and either magnetic permeability or specific inductive capacity. The dimensions of the two latter in terms of mass, length, and time are not known, but the dimensions of their product are known. They are those of the inverse square of a velocity. Hence it is not improbable that the dimensions of the separate quantities may ultimately be discovered. In any case, however, no difficulty arises in practical calculation, for the two quantities mentioned enter into our equations merely as numerics, namely as the ratios of their values for any substance to their values for air. Hence the question of their expressibility in terms of mass, length, and time does not arise. Temperature, permeability, and inductivity are intensive. Hence it has been proposed to replace them by entropy and electric charge, both of which have extensive magnitude.

Resuming our discussion, it should be observed that in measurements of mass we are always determining

${ }^{1}$ See, e.g., B. Russell's Our Knoweledge of the External World, Lect. IV. 
mass-ratio. We select any convenient standard of mass and find how many such parts would make up the mass of the body we are considering. Now the relations between force and mass (or inertia) are expressed in the fundamental postulates of mechanical science, commonly known as Newton's Laws of Motion. From the second law it appears that the ratio of the masses of two bodies is inversely as the rates of change of their velocities produced by equal forces acting on them, or, if the forces are impulsive, inversely as the sudden changes of velocity produced. The third law states that exactly equal forces (though in opposite directions) act on the bodies in the case of any interaction between them. In particular, in the case of impact between the bodies there exist equal, opposite, impulsive forces. Hence the theoretical measurement of mass-ratio (which is all that can be measured) depends on the observation of changes in velocity. Hence measurements of mass reduce to measurements of time and distance. Thus the significance of quantitative notions in physical science depends ultimately on the fact that we are here dealing with things which may be considered as made up of parts, namely times and distances. The parts selected will be the units of time and distance in terms of which we measure.

We are thus led to the conclusion that physical calculation in connection with the world we perceive is rendered possible by the fact that the nature of the object of sense-experience is such that it may be considered (at least to a close approximation) as made up of parts standing in spatial and temporal relations. Hence quantitative notions are to this extent applicable to wha twe perceive in sense-experience, and conse- 


\section{The Notion of a Deterministic System}

quently the terms "deterministic" and "non-deterministic" are significant of what is thus perceived.

It does not follow, of course, that sense-data form a deterministic system. As we have previously stated, the physical system is deterministic provided the fundamental postulates of physics are granted. But the physical system, based as it is on the supposition that the object of experience is made up of parts standing in certain relations, is only an approximation. In actual sense-experience the object is a presented whole, one and indivisible. The object perceived by each subject is unique; but in reflective analysis, which is discursive, we are compelled by the limitations of intellect to regard all objects of experience as having at least one common characteristic, namely that they are made up of parts (termed sense-data) standing in spatial and temporal relations. That this conception is a close approximation to actuality is shown by the fact that under suitable circumstances we can successfully predict, by adopting it, what we shall perceive at future times, provided we are attending appropriately at those times. But we are only approximating, and the perception by which we verify our calculation only approximates to the predicted result to the same order as our original data for calculation approximate to the perceptions on which they are based.

Moreover, it must be remembered that the object of experience is qualitative. Qualities cannot be dealt with by calculation directly, though it may be possible to deal with them indirectly by correlating them with quantities. But even in this case we have no guarantee that the quality which one person correlates with a certain quantity can be considered as similar to that 
which another person correlates with the same quantity. The appearance which I call "red," for example, may be qualitatively quite different from that which you call "red." If such were the case, it would be possible at most to predict approximately one's own sense-data. One could not predict in imagination those of other people. And there is also the further point that it seems probable that even for one person each perception is qualitatively unique. This being so, any prediction of one's own sense-data would be of the roughest character.

Evidently, then, there are strong presumptions against the view that sense-data form a deterministic system. The question will not be pressed, however, for we are not here concerned to come to a definite conclusion on that particular aspect of the problem. But we may note that the material world can only be regarded as a deterministic system, if it be isolated from mind. Prima facie, however, mind interferes with the course of matter, and it remains to decide whether that interference is determined or not. This brings us to our next point, the application of determinism to the mind.

\section{Determinism and the Mind}

In investigating the problem as to whether the notion of determinism is applicable to the mind, we must first of all be quite clear on the meaning of the latter term. In other words, what types of facts are to be included under the heading "mental"? The most satisfactory and unambiguous definition of "mental" is that which pertains to the subject as distinguished from the object of experience. This limits us to feeling and the 


\section{I38 The Notion of a Deterministic System}

various forms of subjective activity, such as thinking, willing, desiring, etc. All such modes of activity may probably be reduced to the single activity of attention, the differences between them consisting in the different types of objects respectively attended to. There seems, however, to be an exception in the case of volition, which appears incapable of reduction to attention alone in so far as it implies motives ${ }^{1}$.

Let us approach the problem before us by referring back to the first example given by $\mathrm{Mr}$ Russell as illustrating a deterministic system. We saw that it represented a world which the actual universe must resemble more or less closely if it is deterministic. In that example the probability is considered of there being a number of mental determinants of the world. "Given $n$ states of a given man's mind," it might be possible to calculate the state of the whole universe, mental and material, at any given time, past, present, or future ${ }^{2}$. Now there are two points in the phrase "Given $n$ states of mind" which demand criticism. In the first place, what exactly is $a$ state of mind? It is evidently impossible to differentiate precisely one state of mind, which might accordingly be "given." State of mind, if it means anything, must mean the subject acting and feeling. But the subject is one-an individual entity. We cannot significantly suppose a section of his activity and feeling "cut out," so to speak, as one state of mind. His active existence is an indivisible whole. We cannot even conceive of sections of it. Nor can it be objected that we can at least say that some actions are before others, thus making a time-basis on which such a conception might

'See, e.g., Dr James Ward's article "Psychology" in the Ency. Brit. (Sec. 9).

${ }^{2}$ See p. 122 above. 
be founded, for in all cases the temporal sign attaches not to the activity itself, but to the changes in the object of experience of which the activity is the ground. We only arrive at the perception of temporal relations in the object of experience through our activity in differentiating that object; and we cannot conceive of a "section" of that activity by attempting to correlate it with a temporal section of the object, for it is the activity of the one individual subject in whom it is evidently meaningless to try and distinguish temporal relations. We might just as well (and just as meaninglessly) attempt to conceive a spatial section of activity on the ground that we attend to objects situated in different places.

A second point now arises. Even if it be granted that there is something actually corresponding more or less vaguely to the notion of a state of mind, what is meant by saying that it is "given"? Evidently it cannot be given as an immediate datum, that is, as an object of acquaintance. For clearly one subject cannot be acquainted with the feeling and activity of another subject. Nor can he be acquainted with his own feeling and activity. The latter would imply that the subject (not as conceived, but in his actuality) was object of his own knowing, which is impossible. Yet we certainly have knowledge about activity and feeling. How does this arise? It is based on what may be called realization. We realize our own activity and feeling, for it is we who feel and are active. Such realization is not itself knowledge, for it implies no object, though the proposition asserting its existence is, of course, a piece of knowledge by description. Thus a 


\section{I40 The Notion of a Deterministic System}

"state of mind" cannot be given as an immediate datum, nor can it be described with any adequacy.

Let us even grant, however, that there is some sense in which a "state of mind" may be supposed to be given. We must then enquire as to the form in which it is given. In considering the attempts of the Laplacean calculator to discover whether the universe is deterministic or not, we saw that it would be necessary to formulate general laws referring to matter, mind, and their interaction (real or apparent), based on exhaustive observation. From these it might be possible to construct, by calculation, a functional relation of the type considered, and thus to establish the determinism of the universe. If it were found possible, in the course of the calculation, to eliminate mental factors, the universe would have at least one set of purely material determinants. As, however, we cannot avoid introducing mental factors at the outset, it is evident that if the calculation is to be possible at all, a "state of mind" must be capable of being given in a quantitative form. Otherwise, we are debarred at the very beginning from attempting to construct our functional relation; debarred, not by practical difficulties of computation, but by the fact that nothing can be inferred from such a relation as to things of which quantitative notions are not significant, and conversely, that if such things exist in the universe, the attempt to construct a functional relation which shall be significant of the whole universe, is meaningless. For it implies calculations involving things to which calculation is not applicable at all.

Moreover, if material determinants are sufficient, we ought to be able to predict future mental facts 
simply by determining the material state of the universe at that time. But the material state could only be specified quantitatively, and how would it be possible to correlate it with mental facts if quantitative notions are in no way significant of the latter? We might conceivably be able to predict fairly accurately the sense-data which a given man would perceive at that time, provided he should be attending appropriately. But how are we to foretell whether he will be attending appropriately, unless attention is susceptible of calculation, that is, is quantitative in nature? It does not help us in the least to assume that to a certain state of brain, a certain "state of mind" corresponds, unless we can state precisely the nature of the correspondence. Nor can it be urged that just as we correlate a sense-datum such as "a patch of red" with something quantitative such as a wave-length, so may we perhaps be able to correlate mental facts with something quantitative. For the concept of a wave-length is itself merely a construction of sense-data, and ultimately depends on the fact that it is possible, at least approximately, to apply quantitative notions to the object of sense-experience by regarding it as made up of parts standing in spatial and temporal relations. Unless some such approximation is also possible in the case of activity and feeling, it is hopeless even to attempt to correlate them with objects of which quantitative notions are significant.

The crucial test, then, lies in discovering whether quantity is significant of feeling and activity or not. Let us consider feeling first. Certainly we talk as if the vaguer quantitative notions might apply to it. We speak of being more or less pleased or pained. But 


\section{I42 The Notion of a Deterministic System}

obviously we cannot get a certain pleasure by addition of other pleasures. A state of "being pleased" is one indivisible thing, not a collection of parts which are themselves states of "being pleased." It will probably be urged that pleasure and pain are analogous to quantities having intensive magnitude. We saw, however, that the latter are not strictly quantities at all, and, in fact, the only reason we ever apply the term to them is that we can correlate them with true quantities. But the basis of this correlation is the fact that both the terms in it are reducible ultimately to sense-data, of which they are constructions, and sense-data may for most purposes be regarded as parts of the object of experience standing in spatial and temporal relations. No such correlation as that mentioned is possible in the case of feeling. For what is the necessary extensive quantity? Certainly not something physical, for there would then be no common basis such as we get in the case of two correlated quantities which are both physical. Feeling is not a sense-datum. We do not perceive feeling. We feel. Probably we only use even the vaguer quantitative terms "more" and "less" of pleasure and pain, which are purely qualitative, because we instinctively try to objectify them by comparing them to physical stimuli possessing intensity. Thus if we are to specify feelings quantitatively at all, it must be by correlating them with some other mental factor which is quantitative. This brings us back to the original question. For the only other mental factor is activity, and this we must now consider.

When we come to deal with the various modes of subjective activity, we find that the hopelessness of 
the attempt to make quantitative notions significant of mental facts is more clearly demonstrated than ever. Again we use the vaguer quantitative terms. We "concentrate" our attention. We are "more absorbed" in some things than in others. But here the quantitative reference is evidently to the objects to which we attend. For example, by concentration we simply mean that we confine the portion of the object of experience termed "the focus of attention" to very narrow limits. Hence the quantitative reference is strictly to that portion of the object, and not to the attention itself. Moreover, attention depends on interest. Clearly interests are not quantitative. They are not made up of parts which are themselves interests.

Similar considerations make it evident that what we have just said is true of all mental activity. A willing is not the sum of parts which are themselves willings. A thinking is not made up of thinkings, nor a desiring of desirings. And in general, we may say that the notion of an act of attention as being made up of parts which are acts of attention, is quite meaningless.

Any attempted analogy of mental activity with quantities having intensive magnitude is of no help whatever. As we have seen, if there are entities actually corresponding to the concept of such quantities, they must really be more like abstract qualities. But this is not the most serious objection. To insist on a previous point, the idea of a quantity only becomes applied to these concepts because we can correlate them with true or extensive quantities. On what is this correlation based? An intensive magnitude is always a function of extensive magnitudes. Density is the 


\section{I44 The Notion of a Deterministic System}

ratio of mass and volume; temperature, of energy and entropy; permeability, of magnetic induction and magnetic intensity. In each case the ratio is that of two extensive quantities, or of two quantities which may be simply and immediately correlated with extensive quantities. Now extensive quantities are logical functions of sense-data. Hence all quantities we thus deal with start from the same basis. From sense-data we construct extensive quantities, from extensive we construct intensive quantities. That is the principle of the correlation. It is not a correlation of two things springing from utterly independent sources. It follows from the fact that we may consider the object of experience as composed of parts standing in spatial and temporal relations.

We cannot, however, arrive at feeling and attention by constructions of sense-data, especially as the constructing process itself involves attention. Therefore we cannot possibly correlate mental facts with any material quantities, whether the latter possess intensive or extensive magnitude. Hence, if quantity is to be significant of any mental factor which is not itself directly quantitative, it must come about by correlating it ultimately with some mental factor of which the notion of extensive quantity is significant. But we have seen that this notion is not significant of any mental factor. Thus it is impossible to effect the necessary correlation in any way whatever.

Evidently the foregoing is summed up in the statement that the feeling, acting subject of experience is an absolute, indivisible unit. The notion that the subject is made up of parts (themselves subjects) is without any meaning. Nor is it possible by any means 


\section{Determinism and the Mind}

to correlate his feeling and activity with something possessing magnitude. Therefore quantitative notions are utterly without significance in application to the facts of mind. Hence it is impossible to construct a functional relation of the type considered which shall take account of mental factors, and it is impossible, not because of any practical difficulties of calculation, but because the existence of a functional relation in such circumstances is contrary to the very idea of such a relation.

The subject, then, is not determined. Strictly, it is neither true nor false to say that the subject is determined. It is meaningless. But the universe comprises subjects, and so no functional relation can exist which is descriptive of the state of the universe as a whole. Therefore the universe is not a deterministic system.

\section{The Problem of Free-Will}

The application of the results of the preceding section to the problem of free-will is more or less obvious. There are one or two points of importance, however, which deserve notice. In the first place, it is necessary to give a clear meaning to the term "free." Probably confusion has often arisen in past discussions on the subject by regarding this term as the opposite of "determined." That view is incorrect. The opposite of " determined" is "undetermined." Both these terms apply to systems of which quantitative notions are significant, i.e., to systems whose states at any given time can be described in terms of quantities which are theoretically measurable. As we have seen, if, from observations on the state of such a system at certain

R. S. P. 


\section{I46 The Notion of a Deterministic System}

times, it is theoretically possible to construct a functional relation giving its state at any time (at least, within a given interval), the system is "deterministic," or "determined." If, on the other hand, the construction of such a relation cannot be performed, the system is " undetermined." Neither of the terms thus defined, however, is applicable to systems of which quantitative notions are not significant; and it is to these systems that the term "free" may properly be applied. Hence, since quantity is not significant of volition, the will is free, or rather we are free in willing.

Clearly, though, volitions are not utterly chaotic. There is a very definite sense in which they are intelligible and coherent. But the coherence is not of a logical kind. When we say that a man's actions are intelligible, we mean that we understand them. The basis of this comprehension is not formal and abstract, but concrete. It is not the laws of logic, but the nature of the self. The self is purposive; its striving is towards betterment by entrance into a completer harmony with the active beings that surround it. Thus a man's actions are intelligible to us, when we realize that they are the expression of purposes analogous to our own. In that Realm of Ends (to use Kant's expressive phrase) which constitutes the world as we know it, we find, not logical determinism, but teleological guidance.

The category of End or Purpose is subjective. It cannot be reduced to any other category, nor can the things to which it applies be subsumed in any way under the notion of quantity. Purposes, intentions, and motives are not measurable. They are not capable of description in quantitative terms, nor can they be cor- 
related with quantities. Yet their introduction into our explanations of certain facts is inevitable. We cannot explain the coming together of the parts of a watch or of a motor-car simply in terms of the motions and configurations of the molecules composing the brain and nervous system of each person taking part in the manufacture. For such creative work is the very opposite of what is implied in the laws of molecular physics. There we have a constant breaking down and levelling, not a building up. Any attempted explanation of a work of the kind considered, by the help of purely physical categories, inevitably leaves us dissatisfied, with an irreducible minimum which cannot be thus explained. This residual factor is the purpose for which the watch or the motor-car is designed; and the fact that quantity is not significant of purpose emphasizes the truth of the statement that the subject, in the exercise of the power he possesses of guiding the course of phenomena, is free, in the fullest sense of that term.

\section{Summary and Conclusion}

At the outset of our enquiry into the problem of determinism, we saw that it would be necessary to elucidate a clear meaning of that term, owing to the fact that many of the difficulties which have arisen in the past have been purely verbal in nature. Consequently we decided to attempt to formulate a satisfactory definition of a "deterministic system," and one which should correspond as far as possible to the essence of the generally accepted meaning of that phrase, by discovering the bare necessary and sufficient conditions which 


\section{I48 The Notion of a Deterministic System}

such a system would have to satisfy. The notion of purely physical compulsion was rejected; for although our actions, in so far as we are part of the material world, must conform to the laws which hold in that world, yet the mental factors which give rise to those actions are not prima facie governed by purely physical conditions. In fact, the reverse appears to be the case. Even should it turn out, however, that mental "events" are determined in some way by physical events, it seemed probable that the facts would be capable of more abstract statement than would be involved in the assumption of some definite type of "compulsion"-whatever that may mean.

The statement that "the future will be what it will be" was next considered. Analysis showed that all such propositions reduce to the assertion that being is determinate. Pure being is identical with Nothing. Accordingly, it appeared necessary to call attention to the distinction between the terms "determinate" and "determined." To say that the future will be what it will be, is to say that the future when it comes will have a definite nature, i.e., will be determinate. This is very different from saying that the future is in fact determined now. The latter implies that if only we had sufficient data, we should be able to predict the state of the universe at any future time.

The last consideration leads at once to the necessary condition which a system must fulfil if it is to be deterministic. The formal statement of that condition is due to Bertrand Russell. If a system is deterministic throughout a given interval, there must exist a functional relation giving its state at any time during that interval, 
involving as data certain events at certain times, these events being defined as the "determinants" of the system. A system might have more than one set of determinants. If one set is purely material, the system is "mechanical." The mere existence of the said functional relation is the one necessary and sufficient condition that the system should be deterministic. The particular type of inner nature or "mechanism" to which the behaviour of the system described in the functional relation is due, is not referred to in the formal definition, nor is it necessary to refer to it.

The illustrations given by Mr Russell, expressed in terms of the methodological principle known as psychophysical parallelism, depicted a world of a type to which ours must closely conform if it be deterministic. It appeared that the state of such a world at any time might be given by a functional relation having purely material determinants, or purely mental determinants, the latter consisting in a number of "states of a given man's mind." One important characteristic of a world of this kind, pointed out in the second illustration, is that it might be called "mechanical " or "teleological," according to the point of view-" mechanical," because one of its sets of determinants is purely material, "teleological," because purposes exist in it which may be realized. It was seen that the view which regards the universe as a deterministic system, receives powerful support from the fact that the conception of such a system has been applied most successfully to the world as dealt with by physical science.

Analysis of the foregoing statement of determinism showed, in the first place, that the form of the functional 


\section{I50 The Notion of a Deterministic System}

relation should be included as a determinant of the system. The determinant data of the functional relation alone could give us no information about the state of the system. We must also know the exact relation between them, i.e., the form of this function into which they enter as constituents. Assuming that we had the intellectual grasp of Laplace's imaginary calculator, we saw that in order to construct a functional relation descriptive of the state of our universe at any time, we should have to start by drawing up a number of general laws, based on exhaustive observation and experiment, dealing with mind, matter, and the interaction between them. No initial assumption about the latter is permissible. We should then have to try and combine our general laws by calculation, in such a way as to be left with the required functional relation. It might be possible to effect this combination in more than one way, the final function involving correspondingly different sets of determinants. In particular, one appropriate elimination might give a set of purely material determinants -another might give a set of purely mental determinants. In any case, it is evident that if the construction of the functional relation is to be conceivable, the general laws originally drawn up must be capable of expression in precise quantitative terms. Hence the words "determined " and "undetermined" can only be significant of systems of which quantitative notions are significant, i.e., of systems whose state at any time can be described in terms of theoretically measurable quantities. If a functional relation can be constructed for a system of this kind, the system is determined; if not, it is undetermined. But unless the state of the system can be described 
thus quantitativel neither of these terms is significant of it.

The quantitieswith which we have to deal are of two kinds, those aving extensive, and those having intensive, magnitue. It appeared, however, that the term "quantity" nnight well be applied only to the former. It becores applied to the latter, because in every case such a uantity may be specified numerically by correlation wit extensive quantities. If, however, we try to imagin something actually corresponding to these so-called"intensive" quantities, we should have to conceivethem rather as abstract states or qualities. Tempeature may be taken as a typical example. Accorngly it follows that quantitative notions are only aplicable ultimately to systems the constituents of whis are such that any finite portion of them may be consicred as made up of a finite number of finite parts. Wether the process of dividing can be carried on to an vizimate conclusion is another matter which is irrelevantio the point here considered.

It is now appaent why the purely physical world may be regarded a deterministic. Physics is based on Mechanics, and theogically prior concepts of Mechanics are mass, distance and time-interval. Analysis shows that all physical masurements (including that of mass) reduce finally to reasurements of distance and time. Hence the fact thi quantitative notions are significant of the physical wild depends ultimately on the fact that the object of eperience may for most purposes be considered as mac up of parts (sense-data) standing in spatial and tempral relations. This view, however, is only a very close oproximation, for in actual sense-ex- 


\section{I52 The Notion of a Deterministic System}

perience the object is one and indivisible. Hence it does not follow necessarily that perceived phenomena form a deterministic system, and this more especially as the qualities perceived cannot be specified, but merely indicated.

It remained to apply the test for determinism to the facts of mind. It was agreed to restrict the term "mental" to its strict meaning of "that which pertains to the subject as distinguished from the object of experience." Accordingly we had to consider two factors-feeling and activity. The different modes of the latter reduce essentially in every case to attention, any particular mode being characterized by the particular type of object attended to.

Reverting to Mr Russell's illustration, we selected for criticism the phrase "given $n$ states of mind," which expresses the possibility of the existence of a set of mental determinants of the universe. In the first place, there appeared a difficulty in assigning any definite meaning to " $a$ state of mind." The oneness of the subject is so absolute as to render meaningless any attempt to split up his feelings and activities into theoretical actions. Secondly, even supposing this obstacle to be surmounted, a fresh difficulty appears when we try to discover how a "state of mind" can in any sense be "given." For it cannot be given in acquaintance even to the subject to whom it refers, seeing that it is not object of knowledge for that subject, although realized by the latter. It must therefore be supposed to be given by description based on that realization. A third difficulty then arises as to the form which the description must take. If it is to be of any 
use in leading to one of the general laws from which a functional relation may be constructed, or if, given that functional relation, it is to be capable of substitution therein, it must evidently be described in precise quantitative terms. For this to be possible, quantitative notions must be significant of it, and hence we are finally led to the test which lies in discovering whether feeling and activity can in any sense be considered to be made up of parts.

In the case of feeling, i.e., pleasure and pain, it is true that we use the vaguer quantitative terms such as "more" and "less." This, however, is because of a fancied analogy with intensive quantities. Feelingdifference in respect of intensity is purely qualitative. It can in no way be correlated with true, or extensive, quantity, as can intensive quantities. For in the latter case the principle of the correlation is the fact that the conception of both types of quantity springs from a common root, namely sense-data. Quantities such as volume, mass, and energy, having extensive magnitude, are constructions of sense-data. Quantities such as temperature, density, and magnetic permeability, having intensive magnitude, are functions of extensive quantities, and hence ultimately constructions of sense-data. But we cannot construct feelings from sense-data, and the same consideration applies to subjective activity. Hence there is no basis whereby we can correlate mental factors with any of those intensive or extensive quantities which may be measured by observation. Nor are these mental factors themselves quantities. A pleasure is not the sum of parts which are themselves pleasures. The case is, if anything, clearer still in the case of activity. 


\section{I54 The Notion of a Deterministic System}

Here the use of the vaguer quantitative terms evidently has a reference to the object attended to, and not to the attending itself, as when we speak of "concentrating" the attention. Moreover, it is obvious that an act of attention is not made up of parts which are themselves acts of attention. Willing is not composed of willings, desiring of desirings, nor thinking of thinkings. Even granted, then, that such a thing as a "state of mind" may exist in any definite sense, the act or feeling in which the state consists is an absolute indivisible unit. Therefore quantitative notions are not significant of mind, and, consequently, the terms "determined" and "undetermined" are not significant of mind either, nor of any system in which minds exist, considered as a whole.

It follows immediately that it is strictly neither true nor false to say that the will is determined. It is meaningless, in view of what the will is. In willing we are therefore "free" in the fullest sense of the word. But that freedom is not manifested in chaos. The acts of a man are intelligible to others in virtue, not of a logical, but of a teleological coherence. We have, in fact, not logical determinism, but purposive guidance of phenomena. Nor can these purposes and interests be weighed out quantitatively and numerically ear-marked for calculation of the future. Given the end which it is purposed to bring about, we may approximately predict the probable actions whereby the end will be realized. But how to foretell the purpose? It is hidden in the individuality of the man. Man is free-free in his thoughts and aspirations, free in his intercourse with his environment, free to make the best or the worst of what he finds therein. 


\section{V \\ THE INTENSITY OF SENSE-DATA}

Sense-DAta are commonly regarded as possessing both types of magnitude which pertain to quantities, namely intensive and extensive. Not only do we speak of sense-data as differing from one another in their qualitative aspect, but as regards their quantitative aspect, we say they differ in respect of extensity and of intensity.

Now it is an undoubted fact that sense-data possess extensive magnitude. It is on this, indeed, that all the measurements of physical science depend. The latter reduce ultimately to the observation and measurement of spatial and temporal features; and accordingly the possibility of physical measurement depends on the fact that, for most purposes, the presented object of individual experience can be analyzed into parts (sense-data) standing in spatial and temporal relations, and possessing spatial and temporal characteristics such as size and duration, which are quantities possessing extensive magnitude. The commonest illustration of this is the (relative) measurement of the spatial dimensions of an object by visual comparison with another standard object. Thus extensive magnitude is directly given as a quantitative aspect of visual $^{1}$ sense-data, though the

1 Sense-data of other types, e.g., tactical, auditory, etc., also possess extensive magnitude, though the measurement of this magnitude has not been developed to any degree of precision comparable with that attained in the measurement of visual magnitudes. 


\section{I56 The Intensity of Sense-Data}

number expressing any particular magnitude will of course depend on the standard or unit chosen.

On the other hand, it appears at first sight equally certain that sense-data possess intensive magnitude. We speak of one patch of light as being brighter than another, or of one sound as being louder than another; and this implication of "more" and "less" is apparently a sufficient criterion of the existence of magnitude. It would therefore follow that the intensity of a sensedatum is a quantity, and should thus be measurable.

But at this point difficulties arise. We can correlate the intensity of any given sense-datum with a certain physical quantity having intensive magnitudenot directly given, without difficulty. Strictly, however, this affords us no information as to the intensive magnitude (relative or absolute) of the directly given sense-datum itself. The problem is, then, to effect the direct measurement of the latter. Evidently we can only expect to determine the relative intensity, and for this it is necessary to carry out a comparison of different sense-data in respect of theirintensity. With the numerousobstacles encountered by such an attempt it is unnecessary to deal at length - they are only too well-known. It is not simply the impossibility of fixing a standard which shall be the same for everyone that is the chief difficulty, nor even the impossibility of fixing a standard which shall be the same for the same person at different times. Such obstacles might be surmounted by methods analogous to those adopted in fixing a standard of extensive magnitude which shall be the same for all persons at all times. Methods of this kind consist essentially in certain correlations of sense-data. They must not be 
confused with methods such as that attempted by Fechner. The principle adopted by him was simply the expression of intensity in terms of a standard intensity. He tried to avoid the difficulty attending a choice of the latter by fixing on the minimum perceptible difference as unit. But not only is this complicated by the fact that the minimum is different for different people; it also involves the assumption that it is the same for the same person quite independently of the rest of the presented field-quite independently, for example, of the total intensity of the particular sensedatum ${ }^{1}$ whose change is being observed. For such an assumption there is no warrant whatever.

The great difficulty lies, however, not in fixing a standard, but in making use of it when fixed. We cannot say, for example, that one sense-datum is twice as intensive as another merely by comparing them. In the case of intensity there is no ground for such a judgment apart from a standard, but when the latter is chosen the difficulty recurs in attempting to compare other sense-data with it. There seems to be no definite meaning to be attached to such commonly heard statements as "This is ten times as bright as the other." If we try to make an exact statement, there appears nothing to go on, nothing to get hold of, as it were, in estimating that the intensity of one sense-datum is so

1 The minimum change in stimulus necessary to give a perceptible difference in the corresponding sense-datum is proportional, as Fechner discovered, to the total stimulus employed. But he could only infer a quantitative relation between stimulus and sense-datum by assuming the least perceptible change in the latter to be a constant, independent of the actual intensity of the sense-datum. 
many times that of another. Only in judging sameness of intensity are we on ground that is at all safe.

It appears, then, that we have two facts to account for which seem to imply more or less contradictory results. In the first place, we use quantitative phraseology in speaking of the intensity of sense-data; yet, secondly, the direct measurement of the latter is impossible. Moreover, not only is it a fact that we cannot judge (say) that the brightness of one patch of light is double that of another, it is doubtful whether any real meaning can be attached to such a statement at all. To realize this, it is only necessary to try to think out such a case and to arrive at some definite meaning of the statement; and, be it noted, a comparative statement of this kind involves no reference to any particular unit.

Now most extensive quantities and all intensive quantities with which we deal are functions of two directly given extensive quantities. The latter are length and duration. With these we start, and from them we construct first of all other extensive quantities (area, volume, etc.) and later intensive quantities (density, etc.). The latter are thus without exception functions of extensive quantities. Indeed, physically speaking, we cannot conceive of an intensive quantity which is not a function of extensive quantities. Yet we cannot say this of the intensity of sense-data, for it is something immediately given in experience. True, the intensity of the physical stimuli is a function of extensive quantities, but that is a very different thing.

We are therefore faced with two alternatives; either the intensive magnitude of sense-data is an ultimate given fact, or else what we call the "intensity" of sense- 
data is not a quantity at all. Now the first alternative is quite hopeless. For the intensive magnitude of a sense-datum cannot be given in itself, nor yet by comparison with other sense-data. Asregards their extensity we can compare sense-data immediately. Not so, however, as regards their intensity; in fact, this, as we have seen, is the root of the whole difficulty and contradiction.

It remains, then, to consider the second alternative, namely that the intensity of sense-data is not a quantity at all. Acceptance of this is conditional upon our being able to account for the use of such terms as "more" and "less" in this connection. Yet there seems to be no difficulty about this. We use quantitative phraseology simply because, as regards intensity, we can arrange sense-data in a certain order. But this does not imply that intensity is a quantity.

Consider the case of a man who should see for the first time. Suppose he is shown two screens of equal size, one dully, one brightly illuminated. Provided associated organic sensations could be eliminated (and this is an important point, though quite impossible to carry out in practice of course), would he arrange the two screens in any particular order of "greatness," being simply told to do that? It is doubtful whether he would even realize in respect of what they were supposed to differ in greatness. If, on the other hand, he were shown three screens, he would be able to arrange them in order, though probably without making any assertion as to whether the order were ascending or descending.

But upon what, then, is this order based? We are 
now committed to regarding the intensity as a quality and we must accordingly suppose the sense-data to be arranged in order of qualitative similarity as regards their intensity, just as we arrange patches of light in order of qualitative similarity as regards their colour. But the basis of such judgments of similarity probably rests on the movements of attention'. In general we arrange lights in a certain order because we have previously attended to them in that order, e.g., when the gas is turned up. Or if the differences are not continuous (as in the case of the three illuminated screens), our judgment is based on the amount of continuous change in the accommodation of attention in passing from one to the other.

Perhaps the best illustrative analogy of the foregoing is that of the pitch of a note. We talk of higher and lower pitch. Yet pitch is not a quantity although we can correlate it with a pure quantity, namely frequency of vibration. We talk of higher and lower notes simply because in respect of pitch we can arrange musical notes in a certain order of similarity. Now according to the results we have arrived at, exactly the same holds of the loudness of notes. Just as notes of the same loudness may differ in their pitch-quality, so may notes of the same pitch differ in their loudness-quality. In other words, brightness, loudness, and all similar "intensities," though commonly regarded as quantities, are really qualities. The order in which we arrange them is the order of their similarity, though probably the use of quantitative terms depends also to some extent on the occurrence of associated sensations having extensive magnitude.

1 Cf. James Ward, Psychological Principles, p. 329. 
We have seen, then, that the paradox arising from the impossibility of measuring the intensity of sensedata and from the difficulty of giving a definite meaning to the quantitative statements involved, leads to the raising of the question as to whether this intensity is a quantity at all. Since all the intensive quantities of which we know are ultimately functions of certain immediately given extensive quantities, we are driven to suppose either that the intensive magnitude of sensedata is something directly given, or else that the intensity of the latter is not a quantity at all. Rejecting the former alternative, and accepting the latter, we found that the application of quantitative terms depends here simply on the fact that we can arrange sense-data as regards intensity in a certain order of qualitative similarity based on the movements and accommodation of attention. The recognition of the fact that the intensity of sense-data is a quality, and not a quantity, would do much to clear up certain branches of so-called "psychical" measurement, besides having an important bearing on certain philosophical questions. 


\section{VI}

\section{IMMORTALITY}

\section{INTRODUCTION}

A cLose inspection of any one of the traditional problems of philosophy reveals in nearly every case a remarkable amount of confusion. This confusion is generally due in part to verbal ambiguities, but the latter by no means give rise to all the difficulties concerned. Usually it turns out that several distinct issues are involved in what appears at first sight to be but a single question. Further consideration shows that as regards some of these issues there is no real problem at all. Others of them are strictly meaningless as generally stated, while to yet others a more or less definite answer can be given.

It is intended here to investigate the general aspects of one of these long-standing problems-the problem of immortality. An attempt will be made to state that problem clearly, to disentangle the various issues at stake in it, and thereafter to show where the real difficulties lie, which of them are insoluble, and to solve as far as possible those which are capable of solution. Particular points, on the other hand, will not be considered. We shall not be concerned, for example, with speculations as to the probable nature of a "future 


\section{Introduction}

life" beyond this present bodily one, should there be such a life.

The question before us, as popularly stated, takes some such form as: "Do we live for ever?" This statement would of course include in its scope not only life after death, but also life prior to birth. From the general point of view precisely the same issues are involved in both these cases. But before going further it is necessary to point out a possible difficulty. What of the "we" referred to in the question? Is it to be supposed that after the lapse of considerable (not to say eternal) periods of time, a surviving individual could in any sense be considered to be identical with the individual as he now is? Evidently we must hereinafter take further account of this matter, for to all appearances the hand of Time lies heavy upon every man, and change (whether it be growth or decay) is one of the commonplaces of existence.

A further point now arises. Is the question "Do we live for ever?" to be identified with the question "Do we exist for ever?" In other words, is existence for individuals such as we identical with living, that is with experiencing? This may be answered in the affirmative. We are essentially experiencing subjects. Our existence consists entirely in our experience. Apart from the latter we are nothing. More, however, will be said on this point later. For the present we may be content simply to emphasize the identity of our existence with our experience and to go on to the consideration of what our problem really amounts to when stated as clearly as possible. 


\section{The Logical Statement of the Problem}

Consideration of the question "Do we live for ever?" shows that the crux of the whole matter lies in the final phrase- "for ever." A definite meaning must be assigned to this phrase. Before this can be done, however, it will evidently be necessary to make some investigation of the nature of time. In fact, the latter is clearly the master factor in the problem. If we can come to a satisfactory conclusion as to the nature of time, a key will have been found wherewith the gate to the solution of the riddle of immortality may be unlocked.

By far the best method of approaching such a task is to carry out a psychological analysis of the growth in the individual experience of that particular type of knowledge which is termed "time-knowledge." It is not proposed to carry out that analysis here. It will be sufficient to give an account of the results at which it arrives and then to estimate the bearing of these results on the main question which confronts us.

In the first place, analysis shows that it is impossible to conceive of the existence of time apart from objects which are, as we say, "in time." That is, time is itself conditioned by those very phenomena the description of which involves temporal ideas. Although we may construct the concept of time as something which subsists of itself, we cannot suppose that there is really such a thing as empty time in which objects come to have their being. What we find as actually given in experience, is the fact that objects have certain peculiar characteristics, such as duration, whichare signi- 
fied by the name "temporal," and that certain types of relation hold between objects, these types also being signified by that name. In other words, what actually exists is not a self-subsistent time, but sense-data within experience possessing temporal qualities and standing to one another in temporal relations. Time-knowledge is then simply the recognition of these qualities and relations within the totum objectivum of our experience.

The individual subject of experience thus arrives at the notion of time by the perception of temporal qualities and relations. But the time to the knowledge of which he in this way attains is purely private. The peculiar characteristics of the sense-data in each individual experience are incommunicable as such, so that each subject has his own private time. We pass, however, by intersubjective intercourse to the construction of the concept of a public or universal time. This is carried out by establishing a one-one correlation between the sense-data of any two individuals. We can then speak of an event in $A$ 's experience as being before, after, or simultaneous with an event in $B$ 's experience. We must, however, carefully distinguish the relation thus established between the two events from the relation between two events both of which occur in the experience of the same individual. In what has so far been said, there appears no ground whatever for regarding the former as temporal in any sense similar to that in which we regard the latter as temporal; nor have we so far any grounds for supposing that the experience as a whole of one individual stands in any temporal relation to that of any other individual. For one thing, it must not be forgotten that the supposed 
establishment of the one-one correlation between the experiences of two individuals really consists for each in the establishment of a correlation between events in his own experience, and the inference which he makes to the establishment of a correlation between his own experience and that of the other is by no means logically necessary, however irresistible it may be practically.

The existence of the subject implies the existence of the object of experience. We meet here a point referred to in the introduction ${ }^{1}$, namely the possibility of the existence of an experiencing individual out of the presentational relation to an object implied in experience. The case of dreamless sleep is sometimes cited as an example of such existence. This particular case will be dealt with in the sequel. For the moment we may confine our attention to generalities. In the first place it is evident that the individual, if he exists at all out of the presentational relation, cannot be described during that phase of his existence as a subject. It thus becomes a question as to whether it is of the essence of the nature of such individuals as ourselves to be subjects of experience. This question may be answered in the affirmative. For us, existing means nothing more nor less than living or experiencing. Take away that presented whole of experience to which all our activities are directed, and those activities (which are themselves partly conditioned by the objects of them) must cease, and ourselves with them. We realize in fact that to abstract a subject from the presentational relation is to make such a fundamental change that he could no longer be considered to be in

\footnotetext{
1 See page $6_{3}$ supra.
} 


\section{The Logical Statement of the Problem 167}

any sense the same individual. This is a theoretical way of stating the fact that we are, essentially and existentially, experiencing individuals.

The fundamental fact is, then, the unity of the individual experience comprising the duality of subject and object, each of which implies the other. The question "Shall I live for ever?" thus becomes "Will there be a time when I shall not exist, i.e., shall not have experience ?" The latter, as we have seen, implies and is implied by the question "Will there be a time when no object is presented to me?"

To assign a definite significance to these questions, we must be quite clear on what is meant by the "time" referred to. Evidently it cannot be my private time, without contradiction. For my private time is conditioned by the objects presented to me, in fact it only subsists at all in virtue of these objects. Hence there cannot be an instant in my private time when nothing is presented to me. The two ideas "instant of private time" and "absence of presented objects" are contradictory in this context. The questions we have mentioned are thus only significant if the time referred to is public time. We must accordingly ask "Are there instants of public time when no object is presented to me?" A reply to this in the negative might not rule out immortality in a certain sense. For it might be said that perhaps there are "gaps" in our existence, as it were. The idea of such "gaps" could, of course, only be significant if interpreted in terms of public time. A further question would arise as to the duration (in terms of public time) of these gaps. Are they in any cases infinite? These considerations need 
not detain us, however, for the general characteristics of the problem are not in any way modified by them.

A final step remains. The concept of public time is but a construction based on the correlation of individual private times. The latter, or rather those characteristics of sense-data which are termed "temporal," are the actualities. Our question therefore, in the last analysis takes the form "Are there events in the experience of others which cannot be correlated with any event in my own experience by the one-one correlation referred to above?" This is the logical statement of the problem in terms of the actual facts, when that problem is viewed from a universal standpoint.

The problem propounded in this form would appear to be quite insoluble. We cannot determine from logical considerations whether the required correlation would always be capable of being established (at any rate theoretically) or whether it would break down in certain cases. Nor can we solve the problem empirically. For the failure of the correlation could never be demonstrated in this way. It would not be known to the individual concerned seeing that it implies his own non-existence, and it could not be investigated by other individuals. For, in the first place, since experience is incommunicable we cannot establish the correlation directly, but must always carry out the process indirectly by correlating different events in our own experience; and, in the second place, we cannot assume that an individual does not exist simply because we are not able to communicate with him, that is because certain sense-data are lacking from our experience which wehave been accustomed to regard as manifesting his existence. 


\section{The Logical Statement of the Problem I69}

The insolubility of the problem as stated above is, however, a fact of but little practical importance. For, as the last considerations have made clear, the significance for the individual of the question put thus, and its answer (whatever that may be), is purely formal and abstract. The absence of the correlation between the subject's own experience and that of other people, could ipso facto not be brought home to him in any concrete way. For that matter, the fact of its presence could not be evidenced concretely to him either, seeing that every correlation which he makes is actually between events in his own experience. To estimate the practical bearing of the matter we must therefore abandon the universal and conceptual standpoint we have adopted up to this point, and come back to the private standpoint of individual experience. Returning then to the question of immortality in the form "Shall I exist for ever?" we shall endeavour to bring out the concrete significance of the question and its answer for the individual. It will be found that the results obtained in this direction are more definite.

\section{Concrete Significance of the Problem FOR THE INDIVIDUAL}

Pursuing our enquiry in this new direction, we must first of all emphasize a fact which is continually recurring throughout the investigation. This is the fact that in experience subject and object each imply the existence of the other. Both begin, continue, and cease to be, together, though whether such terms are here strictly applicable at all is a question for later consideration. 
Now the cessation of the existence of an individual could evidently not be a given fact for any other individual. We can give a meaning to the idea of a broken intercourse between subject and subject, for events occur in our experience which can be interpreted in that way. But no event in our experience gives (nor could it give) us certain information of the fact that an individual with whom we no longer have intercourse has ceased to exist. In other words, the ceasing to exist of another individual cannot possibly be given as an object of our acquaintance any more than that individual himself can be given as an object of our acquaintance.

There is a further and far more important point. Not only could the ceasing to exist of an individual not be a fact for other people, but it would also not be a fact for that individual himself. For his experience, in which consists all those events which are facts for him, comes to an end with himself. What is the status of a fact which is not a fact for anybody, and whether there could be such a fact, we need not pause to enquire. Meanwhile, the conclusion so far reached may perhaps be most easily realized by taking up in imagination a solipsistic position. Such a position is a perfectly possible one to adopt theoretically; it cannot be logically refuted. The events in the experience of an individual take place just as if he were the only existing subject'. This brings out the difficulties involved in assigning a definite meaning to the phrase "ceasing to exist." For

1 The apparent manifestation of the existence of similar subjects can be interpreted on the analogy of dreams, if we stick consistently to the solipsistic position. 
it would have no meaning for the individual subject concerned, and if he were the only subject existing it would therefore have no meaning at all.

It follows from the necessary co-existence of subject and object that there are no "gaps," as it were, in the object of experience. For the subject cannot beconscious of nothing. Any such gap in the object of experience would imply a corresponding gap in the existence of the subject. We have no reason whatever to postulate these gaps, for in any case their presence could not possibly be known. They would not be a fact for anybody. The case of dreamless sleep (if there be such a thing) illustrates the point. Dreamless sleep for the subject concerned means nothing more nor less than the occurrence of an unusual sequence in the object of experience. But there is and can be no absolute break in the sequence. We can draw no inferences of any kind from dreamless sleep as regards ceasing to exist, without making some assumption as to the existence of time as an actual entity, for which we have no grounds whatever. For, as we have seen, the actual fact is the existence of temporal qualities and relations within experience and not of some distinct entity called Time.

This last point contains the crux of the whole matter, namely in actual fact it is only objects within the individual experience which can in any concrete sense be said to exist at times (or places), these being conditioned by those very objects. The idea cannot be applied to experience as a whole. For the individual the fact is simply his existence-not his existence at such-and-such a time or at such-and-such a place. The 
latter has noconcrete meaning for him atall. Accordingly, all such terms as "begin," "continue," "cease," which have a temporal reference, are really applicable only to things within the object of experience and not to experience as a whole.

We arrive, then, at the result that for the individual the question "Shall I exist for ever?" is devoid of any concrete significance. We might have expected something of the sort from the fact that whatever interpretation we attempt to put on the idea of ceasing to exist, the latter would be nothing for the individual concerned. Putting aside, therefore, the question "Shall I exist for ever?" we can only replace it, if at all, by the question "Do I exist ?"-which is answered in the very asking of it.

The conclusion we have come to makes it clear that all particular questions commonly connected with the problem of immortality (such, for example, as the question of a future life out of this body) should strictly be put in the form "Are there such-and-such elements in my experience?" no temporal mark of any kind being attached to the proposition. In other words, ultimately we must consider experience as the unity it actually is. Ultimately we cannot regard experience as made up of parts termed "sense-data" or "events." Experience is not a series, but an indivisible unity. What is commonly represented and referred to as its "unique" continuity, is really its unity. Before summarizing, therefore, it will be worth while to consider this point briefly, for, in the first place, it is of the utmost importance in all philosophic thought directed to final problems, and, secondly, it draws together all the threads in the 
particular problem we have been considering. In the light of it our results receive their fullest interpretation and justification.

\section{The Unity of Experience}

The employment of analysis in any given case implies that the ideas of whole and part can be adequately applied in the matter under consideration, for analysis consists essentially in the discovery of the "parts" (whether terms or relations) of a given "whole." In the course of philosophic enquiry we sometimes have to deal with unities to which the ideas of whole and part are not ultimately adequate. Such unities are by their very nature indivisible entities.

We find among unities of this type, the subject and the object of individual experience. It is frequently granted that the subject is an indivisible entity, but the same is far more rarely admitted of the object. We shall therefore confine our attention chiefly to the latter.

It is necessary to mention at the outset that no attempt is being made to demonstrate that an utter falsification is committed by analysis of the object of experience. But we must recognize degrees of validity; and the analysis of experience cannot be entirely valid. The ideas of whole and part apply with different degrees of adequacy to different entities, and the degree in which they apply to the object of experience is not perfect.

Now it is sometimes said that the object of experience is complex and therefore demands analysis. But surely a petitio principii is involved here. For what is meant 
by saying that an object is complex ? Simply that so soon as we attempt to reflect critically on it, or, in other words, to analyze it, we find that we cannot make a single step without introducing the concepts of whole and part. Now it may be granted that this is true of reflection on the object of experience, but in actual experience apart from any judgments referring to it (except perhaps the existential judgment "It is " the "it" being the totum objectivum) there are no "whole" and "parts," but just a given indivisible unity. Considering for the moment, in imagination, that group of objects which we class as "simultaneous parts" of experience (what is sometimes, but perhaps illegitimately, called an "instantaneous" section of experience), we have in the actual experience not a whole of parts but an indivisible unity. Taking a further step, and including within our view what analysis calls the "time-series" of events, we must again conclude that there is not actually a whole consisting of parts which are events, but an indivisible unity. Accordingly, the individual experience must ultimately be taken account of as just such a unity.

The point may be made clearer by asking what is meant by the term "part." It is said $^{1}$ that the whole is an entity and the parts are other entities which exist or subsist independently of their discovery by analysis, and are real in quite the same sense as the whole of which they are parts. This seems a fair statement of the matter. But if the parts are definite entities which are real in quite the same sense as the whole, they must

${ }^{1}$ See the introductory section to "A Defence of Analysis" by E. G. Spaulding in The New Realism. 
surely be capable (at any rate in conception) of existing or subsisting apart from the whole and at the same time maintaining their nature unchanged. This, however, is the root of the whole difficulty. For in the case of a unity such as the object of experience, we find, on considering the supposed "parts," that we have to conclude that the parts are "coloured," as it were, by the whole and their relations to it; that it is essentially of their nature to be parts of that whole; or that the whole exists in and through the parts, while the parts exist in and through the whole. To such statements it would be difficult to assign a definite meaning while still maintaining the clear significance of the ideas of whole and part. The truth is, of course, that we are discovering the inadequacy of those ideas to the indivisible unity of experience. If we press them far enough in this connection, contradictions are bound to emerge.

What we have said applies most obviously to the case of sense-experience. But we must beware of thus limiting the application. The indivisible unity we have been considering is really what may be called the objective situation which we analyze into objects of sense, objects of thought, etc., though strictly, as we have seen, we must regard it as in no way divisible.

Much of the disputation over the matter we are dealing with has arisen in connection with what is called the "continuity of experience." The exponents of analysis ${ }^{1}$ regard the latter as akin to mathematical continuity, analyzing experience into continuous (or, at least, compact) series of elements termed "sense-data." Lect. V.

${ }^{1}$ See, e.g., B. Russell in Our Knowledge of the External World, 
Their opponents, while attacking the analysis, admit some sort of continuity in experience, and in endeavouring to define it have to assume what they are trying to disprove. This illustrates our point excellently, for the cause of the trouble is that experience does not possess continuity at all (seeing that it has no parts) but unity, and the common reference to the "unique" continuity of experience is nothing more nor less than a tacit admission of its unity ${ }^{1}$.

Finally, it would be well to insist again on the point mentioned at the beginning of our consideration of analysis. Analysis of experience is by no means entirely invalid. It is a necessary and justifiable mode of procedure, provided its limitations and presuppositions are kept in mind. But we must recognize that there are degrees of validity governed by the adequacy of the ideas of whole and part to the matter in hand. In the case of the object of experience, analysis into parts (sense-data, etc.) standing in certain relations in virtue of which they form continuous series, provides a close approximation to the actuality. This follows from the fact that the results of calculation based on the analysis can be verified empirically, within limits. But it must not therefore be assumed that the ideas of whole and part are perfectly adequate here, for in any case the results of calculation would approximate to actuality in the same way and to the same order as do the data (yielded by analysis) from which the calculation starts. If we press on far enough, however, difficulties arise

1 The writer has dealt with this question in the essay entitled "Scientific Method in Philosophy and the Foundations of Pluralism," Section VI. 
which make it clear that the conception of the object of experience as a whole of parts consisting of terms and relations in which the terms stand, is not an entirely adequate one. Ultimately therefore, we must consider the object of experience as the indivisible unity it actually is.

The bearing of these facts on the problem of immortality now begins to be apparent. The unity of the individual experience comprises the duality of subject and object each of which is an indivisible unity. Not only then do time-relations apply in any case simply to parts within the object of experience as opposed to that object of experience as a whole, but the judgments on experience which yield analytically these parts and their time-relations are not ultimately adequate. Hence we have absolutely no grounds whatever for postulating a Time in which experiences actually exist-and this in addition to the fact that even were our analysis quite valid, we should have no reason at all to assign to experience as a whole the temporal characteristics and relations of the parts. Consequently neither of the terms "begin" and "cease" has any valid significance when applied to experience considered as the indivisible unity it actually is. Such questions as "Shall I exist for ever?" need therefore no longer trouble us, as they have ultimately no meaning, and the related question which replaces them, namely "Do I exist?" and which is certainly valid, provides its answer, as we have said, in the very asking of it. 


\section{Summary and Conclusion}

The problem of immortality, no less than the other traditional problems of philosophy, exhibits when analyzed a number of distinct issues which have become confused in most treatments of the question. Accordingly it was considered necessary to obtain a clear statement of the problem to find which of the supposed issues at stake are really significant, and to determine those points on which a definite solution may be reached.

In the first place, we found that in order to state the problem clearly it was necessary to assign a definite meaning to the phrase "for ever," in the question "Shall I exist for ever?" The temporal implications of the phrase required a brief investigation of the nature of time, which led to the result that in actual fact there is not a definite independent entity, "Time," in which objects have their being, but certain unique characteristics (named "temporal") of the objects within individual experiences, and certain unique (temporal) relations subsisting between those objects.

On these temporal qualities and relations the concept of the private time of the individual is based. By oneone correlations between "simultaneous" events in the private times, public or universal time is constructed. We therefore found it possible to obtain a logical statement of the problem of immortality involving the idea of public time, and running as follows: "Are there events in the experience of others which cannot be correlated with any event in my own experience by the one-one correlation referred to?" Now since the public time implied in this statement is simply a construction 
based on the individual private times which are the actualities, the statement is necessarily formal and abstract in significance. Consequently the conclusion to which we were compelled, namely that the problem as thus stated is insoluble, loses much of its importance.

It remained to come back to the standpoint of the individual and thence to determine the concrete significance for him of the question in its original form: "Shall I exist for ever?" Now the subject and object of experience imply each other, so that any assertions regarding the existence of the former apply also to the latter. This being so, together with the fact that the actual entities on which the private time of the individual is based are the temporal qualities and relations of the parts within his object of experience, we were led to the result that theabove question has no concrete significance for the individual at all. For there are no grounds whatever for asserting temporal characteristics of experience as a whole. Such terms as "begin," "continue," "cease," are therefore meaningless when applied to that whole.

Finally the central principle which binds together the various aspects of the question, was seen to lie in the unity of experience. The analysis of the object of experience into parts consisting of terms and relations, though a close approximation and a necessity in thought and discussion, is not altogether valid. The ideas of whole and part are not altogether adequate to the object of experience. For the latter is by its very nature an indivisible unity, being, as it is, presented to one subject, that subject itself being an indivisible unity. A fortiori, then, we have ultimately no reasons whatever for 
assigning temporal characteristics to the indivisible unity of experience. The question "Shall I exist for ever?" must be replaced, if at all, by the question "Do I exist?"

We may conclude then, that questions having the most practical significance for the individual are not those relating to his own existence, which concern his experience as a whole, but those dealing with events within his experience. Such, for example, is the question (usually connected with immortality) which refers to the possible meeting beyond this bodily life with other personalities to whom we are attached, and who have passed on before us. Formally, this question would be of the same type if it referred merely to the possibility of meeting again in the future friends who have gone abroad, though the particular determining conditions would of course be different-for example, we should have in the first case to take into account the objective factor of bodily death. No such questions are touched by the general aspects of the problem we have been considering, nor can these aspects possibly shed any light on them. But the consideration of the generalities of the case at least serve a clarifying purpose, and rid the particular problems of elements which, although commonly interwoven into the investigation of those problems in a more or less vague manner, are really quite irrelevant to them. 


\section{VII}

\section{THE RELATION OF MIND AND BODY}

\section{INTRODUCTION}

$T_{H E}$ exact nature of the relation of mind and body is a problem which, although it has occupied the attention in a greater or less degree of the speculative thought of all ages, has come into particular prominence during recent years. The behaviour of those remarkable and unique complexes (unique, that is, in the physical order of Nature) to which the name "organisms" has been given, has been studied in the past by two schools of scientists from two distinct points of view. On the one hand there are the biologist and the physiologist, the latter of whom investigates the individual organism, while the former considers rather the behaviour of species-organisms more or less en masse. Both physiologist and biologist regard behaviour from the objective standpoint of physical science. The material with which they deal is essentially phenomenal. On the other hand stands the psychologist. He is concerned with what is known as the mental "aspect" of the organism. Accordingly his point of view is largely subjective, and this notwithstanding the attitude and methods of recent experimental psychology. Moreover, many (indeed most) of the facts which it is his business to investigate cannot be regarded as phenomenal in the valid sense of that term. 
A large part of physiology's task consists in the observation and description of the functions of the senseorgans, brain, and central nervous system. It traces the path of afferent nerve-currents from the periphery to the sensory centres, and the connection of the latter with the higher centres. Conversely, it observes the passage of efferent impulses from the higher centres to the motor centres and thence to the muscles. Or again it investigates the reflex arc through sensory and motor centres without intervention from the higher centres. But, in any case, at the nerve centres of the brain and central nervous system the physiologist comes to a standstill. He does not proceed to consider how impulses "pass over" into the mind and become content of consciousness, nor how impulses originate in the mind and "pass over" into the brain as the source of bodily activity. For him, at least in so far as he is concerned with his own particular affair, consciousness is epiphenomenal-a mere accompaniment of neural and cerebral phenomena.

The psychologist, on the contrary, approaches the question of behaviour from the mental side. His business is to analyze the individual experience, and to elucidate in particular the subjective factors which determine the growth of that experience. As long as he keeps strictly to his appointed task the concepts of physiology do not come within his scope at all. So far from being epiphenomenal, consciousness is for him the primary concern, while its neural and cerebral concomitants have no interest for him, as psychologist.

Evidently, then, there is a somewhat ill-defined region lying between the realms of physiology and 
psychology with which neither is strictly concerned, a region whose fundamental fact is the mysterious nexus of mind and body. Consequently, this region has been at all times the hunting-ground of the philosopher. As a hunting-ground, however, it can hardly be said to have been happy, for philosophy has at best made but a poor show in its attempt to arrive at a satisfactory solution of the problem of the relation of body and mind. Until comparatively recently, however, this failure has been of no great importance, except, perhaps, to philosophers. But in the course of time both physiologist and psychologist have pushed their respective investigations so far that the categories now employed cannot hope to be interpreted adequately without a satisfactory clearing up of the debatable region referred to. Moreover, such a clearance would possess the additional importance that it could not fail to shed light on the question as to which is the most fundamental standpoint-that of psychology or that of physiology. Physiology and biology, in alliance with physics, would, if possible, interpret the Universe ultimately in terms of matter; while psychology, when it becomes metaphysical, seeks to interpret the Universe in terms of mind. The issue is fundamental for the philosopher.

If we enquire how it is that the efforts of philosophy to solve this problem of body and mind have not been attended by greater success, we find one of the chief obstacles to that success to be of a type which occurs only too commonly in philosophical speculation, namely the lack of a precise statement of the problem. A perusal of the works of various writers on the subject reveals the fact that the terms "body" and "mind" are 
used in many different senses, senses which are frequently vague and ambiguous to the last degree. It is therefore essential, in the first place, to arrive at an exact definition of these terms.

Now it is doubtless true that "body" and "mind" are used with more than one meaning to which a reasonable significance may be attached. The problem and its solution will vary accordingly. This calls for consideration, but it will appear that there is one interpretation of the problem which can claim to be the most definite and significant. After attempting to make this clear, it is intended to approach the problem from the standpoint of a pluralistic spiritualism with a view to obtaining as satisfactory a solution as possible. The general grounds for such an ontological hypothesis will not be advanced, but the latter will be left to be judged by its fruits in this particular case.

\section{True Meaning and Implications of the Problem}

It was formerly an almost invariable custom to regard the relation of body and mind from a frankly dualistic point of view. Nor is this custom by any means a characteristic only of discussions in the past. The dualistic standpoint is adopted, whether tacitly or avowedly, in a considerable portion of the current writing on the subject ${ }^{1}$. The body is regarded as a material thing in an environment of other material

'See, e.g., Dr Bosanquet's Principle of Individuality and Value, Lect. $\mathrm{V}$, in which, although the general position is idealistic, the concepts employed and the corresponding terminology are full of implications which are thoroughly dualistic. 
things, some organic like itself, others inorganic, while the mind is apt to be identified with the content of consciousness. The question then becomes one of the relation between a particular portion of organic matter, and the particular content of consciousness connected with it. Sense-data are thus regarded as mental, and an important part of the investigation consists in an attempt to determine the connection between sense-data and their material stimuli.

Now for those who take up this attitude the concept of matter differs little from that framed by Descartes. Material things are those which obey the laws discovered by physics, and matter is vaguely conceived as a "substance" possessing such qualities as inertia, impenetrability, and extension. The conception of mind in this connection is also characterized by considerable vagueness. The individual mind is regarded more or less as a bundle of sensations, thoughts, feelings, and volitions.

The development of this view is commonly supposed to lead to certain difficulties. Two of these stand out pre-eminently and are worth a brief consideration. In the first place it is urged that mind and matter as thus conceived are such utterly "disparate" entities that the notion of their interaction is inconceivable. Now it may be granted that mind and matter are disparate, but it does not follow necessarily that their interaction is inconceivable. For there seems to be no reason why any two entities should not interact, unless "action" is defined in some particularly narrow way. But it will perhaps be pointed out that whenever a material body is observed to change its state the change is found to 
be due to another material body. This evidently limits the notion of action to the ground of the sequences which occur in the material world. Moreover the statement is not strictly true, and in fact begs the very question at issue. For it cannot be said, for example, that the movements of organic bodies are invariably observed to be due to purely material causes.

In the second place, it is sometimes said that the view we are considering involves a violation of the principle of the conservation of energy. The haziness of the notion entertained by many philosophers of the conservation of energy is truly remarkable. As a matter of fact it is a physical principle which only holds within extremely strict limitations, namely that the systems within which energy is conserved are isolated material systems. Now the body of the organism is not an isolated system, in so far as the organism has a mind which, at least prima facie, influences its body; and if we consider the organism as a whole, it is not a material system, for it comprises mind as well as matter. The principle of the conservation of energy is thus utterly irrelevant to the question at issue. Even could it be shown (which it cannot) that the energy of the body, after allowing for the effect of external material influences, conforms to the principle of conservation, nothing of interest would follow. Controversy rages round the idea of mind as the director of bodily energy, and it is objected that the direction of energy involves in practice the performance of work, which would be manifested as additional energy in the body. But we can only assert that changes in the distribution of energy in a system by material influences imply the performance of work by 
the latter. As to the possibility of changes in distribution of energy due to mental influences, and whether if there are such occurrences expenditure of work is necessary, we can arrive at no conclusion at all.

We are thus bound to conclude that objections such as the above cannot apply to this view of the interaction between body and mind. But the theory fails rather for negative reasons. It does not carry us one step towards the solution of the problem, for it is quite unable to give any intelligible account of the interaction it talks of. It is compelled to fall back on the assertion of the probability of an exact one-to-one correspondence between events in what it calls the mental series, and events in the neural or cerebral series. As to the ground of this correspondence, it is silent. In such a psychophysical parallelism all views based on dualism find their logical end ; and whatever may be said of psychophysical parallelism as a methodological principle, as a metaphysical theory it is an utter failure. The reason for this failure lies in the hopeless artificiality of the dualistic standpoint. Our next concern must therefore be to take up a position in which we shall escape this artificiality.

The life of any being such as ourselves consists in the sensations, feelings, desires, thoughts and acts which go to make up the routine of its existence. All these we group together under the term "experience." The individual experience is a unity, but it is a unity which comprises a duality, for in it are distinguished two fundamental factors. A subject who attends or cognizes, and an object which is attended to or cognized. This duality in unity of experience is the only natural 
position that can be taken up when we begin to philosophize. Not only is it natural, it is also inevitable. For each of us is one of the factors in an individual experience, each of us is an experiencing subject.

The necessity of adopting this position has been called "the ego-centric predicament ${ }^{1}$." Certainly the position is ego-centric; but it can hardly be called a predicament in the usual somewhat deprecating sense of the term, so long as we keep clearly in mind the fact that our position is ego-centric, and refrain from making invalid inferences from the necessity we are under of adopting it.

The realist urges us to try to adopt an "external" point of view, as it were, in our attitude towards the entities with which science deals. He regards our clinging to the ego-centric position as a fruitful source of fallacies. So it is, if we forget the limitations of the position, but not of the kind of fallacies which the realist usually imagines. For what, in fact, do sciences such as physics, chemistry and biology, actually discover? Simply the occurrence of certain sequences in the sense-data presented to us. The discovery of a physical fact lies in perceiving that (say) $A$ is invariably followed by $B$, and the verification of hypotheses built on discovery is always an appeal to the perception of some sequence of sense-data. Now, according to the realist, we must drop saying " $B$ is always perceived to follow $A$," and say simply " $B$ always follows $A$." If the realist is right, the existence is implied of entities essentially akin to sense-data, except that they are not perceived. With the possibility of this we are not here particularly concerned. But at least we are on surer

${ }^{1}$ See R. B. Perry in Present Philosophical Tendencies, pp. 129 ff. 
ground in making the statement "Because $B$ has always been perceived to follow $A$, it is extremely probable that, with appropriate attention, $B$ will always be perceived to follow $A$," than in making the statement "Because $B$ has always been perceived to follow $A$, therefore $B$ will always follow $A ; A, B$, and their sequence being objects quite independent of perception."

Now of course we cannot deny the possibility of the existence of things which have no influence whatever on us, and whose existence can therefore never be manifested to us in perception. The realist seems to feel that in taking up and maintaining the ego-centric position, we shall certainly deny the possibility of the existence of such things. But why should we? In any case, such things could have no concrete significance for us, and provided we are willing to admit the possibility of their existence, and provided also we refrain from denying (since it will not here concern us) that such objects as sense-data may exist unperceived, no exception can be taken to our ego-centric position, nor shall we be committed to any fallacies thereby. In fact, the reverse is the case. For if, with the realist, we assume the existence of unperceived objects otherwise essentially like sense-data ${ }^{1}$, and proceed to act on that assumption, the burden of proof is on us, and a very heavy burden it is. It is strange, in view of these considerations, to reflect that the realist is the first to urge that facts must be ascertained empirically and not $a$ priori.

${ }^{1}$ It is doubtful whether such a phrase as this has any real meaning. See the essay on "The Philosophical Problem raised by the Weber-Fechner Law," Section III. 
The ego-centric position is, then, the standpoint we must take up; for not only is it the position in which we actually are, but also so long as we are dealing with entities which are in fact perceived (whether the perception of them is necessary to their being or not) the position is an unexceptionable one, from which it behoves us to discover as much as possible. And, indeed, the adoption of any other point of view carries with it certain assumptions, as we have seen, which constitute, if not insuperable, at least very great difficulties.

We must now proceed to consider what we really mean when we talk of "material things." The objective field presented to each percipient subject comprises certain shapes, patches of colour, etc., which are at rest or in motion, and between which certain spatial and temporal relations subsist ${ }^{1}$. They are termed "sensedata." As we have seen, the investigations of physical science really consist in determining the sequences which occur among sense-data. But as soon as the naïve stage is passed, physics leaves the immediate data of sense, and proceeds to work with more abstract conceptions such as "material body," "molecule," "atom," etc., and, in so far as space and time are concerned, "point" and "instant." The physical concept which is psychologically primitive is that of a material body, and

1 The writer holds that the presented object of experience, as actually given, is an indivisible unity to which the concepts of whole and part are not entirely adequate. But in any reflection on experience whatever, we are necessarily bound to use those concepts, and we can in fact approximate very closely to actuality by a proper employment of them. This is dealt with more fully in "Scientific Method in Philosophy and the Foundations of Pluralism," Section VI, and in "Immortality," Section IV. 
the physicist now regards sense-data, such as colour and shape, variously as properties of material bodies or as effects on us of which material bodies are the causes,nor is he always quite clear as to what he really believes in this respect. At all events, when the physicist talks of "this square, brown body," he does not generally believe that the body itself is the square, brown patch in his object of experience, but rather something to which the squareness and the brownness are somehow attached as qualities or effects. In other words, he tends to conceive the "matter" of which material bodies are essentially composed as being some mysterious substratum which causes our sense-data or of which the latter are the qualities.

Now at this point the fact must again be insisted on that physical science consists fundamentally in the empirical observation of sense-data and their sequences, and in the construction of hypotheses which are tested by appeal to the perception of certain sense-data. Hence it follows that the laws and hypotheses of physics, no matter what terms be employed in them, must really be statements about sense-data. Thus concepts such as point, instant, material body, and material particle, must actually be logical functions of sense-data if they are to be valid, while matter is itself such a logical function, namely the class of material things. In other words, the material entities spoken of in physics must be regarded not as inferences from sense-data (namely the causes or substrata of the latter), but as constructions of them ${ }^{1}$.

1 The writer considers this to have been unanswerably demonstrated by Bertrand Russell in Our Knowledge of the External World, 
Care must be taken to avoid a certain confusion here. As will be seen later, it seems necessary to postulate a ground of our sense-data. But the point to be urged is that this ground is not what is meant by the matter and material bodies of science and common-sense. The propositions of physics, when analyzed, contain no indications of the existence of entities beyond the immediate data of sense. The terms of those propositions, though they are not in general the data themselves, are functions of them; and the assumption of concrete entities corresponding to these functions, in addition to the data of which they are functions, is a gratuitous and unnecessary one for which there is no reason whatever. This fact, however, is quite irrelevant to the possibility of the existence of a ground of the data which is in no sense akin to the entities with which physics deals, and to which, indeed, physics has no reference at all.

Using the term "matter" in the physical sense, then, it appears that the body as a particular material object, is a certain class of sense-data. This class includes among its members both the sense-data of the individual to whom the body belongs, and those of other individuals by whom that body is perceived. Now there is a certain similarity and a certain difference between these two sets of sense-data. The sight of our own body (as regards those parts which we can see) is essentially similar to the sight which other people have of it. The same holds of touch, with the reservation

Lects. III and IV, and The Ultimate Constituents of Matter in a volume of essays entitled Mysticism and Logic. The above is a condensed statement of the view therein set forth. 
that in exploring our own body we are sensible of that double touch whereby we distinguish it from other bodies. But when we come to musculo-motor and organic sensations, together with that vague presentational mass which goes by the name of "general sensibility," we are concerned with sense-data to which, in connection with our body, there is nothing corresponding in the presentations of other people; though, of course, similar objects are presented to them in connection with their own bodies. On the other hand, there are certain sense-data (corresponding, for example, to our brain) which may be perceived by other people under suitable conditions, but which are never perceived by us.

It appears, then, that the class of sense-data which make up the body of an individual in the physical sense of that term comprises three sub-classes: (I) Sense-data which, though of course peculiar to the individual, resemble essentially the perceptions which other people have of his body. (2) Sense-data perceived only by the individual concerned. (3) Sense-data perceived only by other individuals. Sensations of sight, organic sensations, and observations of the brain, are typical respective examples of members of these three subclasses. Evidently it is members of the first two only that enter concretely into the presented complex which constitutes the body as a physical object for the individual concerned. But the latter in constructing the concept of his body includes in addition the third type, basing his construction on his observations of the brain and internal arrangements of other people, whether he has actually perceived these or merely representations of them. 
We must now leavefor the moment our consideration of the body, to enquire what, from the standpoint we are adopting, can be meant by "the mind." By regarding sense-data as the elements of which material things, the organic body among them, are composed, we have ruled out from the concept of mind, either actually or analogically, all that is contained in the objective side of experience. We are thus left simply with the purely subjective factors in experience, of which there are two-feeling and activity, the latter consisting chiefly and perhaps entirely in attention. In determining the relation between body and mind, where body is interpreted in the sense we have been considering, we must therefore identify the individual mind with the individual feeling, acting subject of experience. By this is meant the concrete Ego, not the empirical Ego or $\mathrm{Me}^{1}$. The latter is but a concept constructed by the concrete Ego-a presented object among other presented objects. What, then, is the relation between the subject and his body regarded as a physical object, when the latter is interpreted in the only valid way?

To this question a definite and simple answer can be given. As we have seen, for the individual, his body, as a definite material object, consists in a certain presented complex of sense-data which differs in some important qualitative respects from other presented objects. Now the relation of this group of sense-data to the individual subject whose body it is, is a presentational relation. Thus, for the individual concerned, the relation of body and mind, where the former is regarded as a physical object and is interpreted as such

${ }^{1}$ See William James, The Principles of Psychology, Chap. $\mathrm{x}$. 
while the latter is identical with the subject of experience, is simply a relation of presentation, the latter being an ultimate relation, the nature of which we all realize. But if we take a more objective standpoint, and include in the body of the individual the sense-data connected with it of other individuals, we are faced with the further question as to the relation of these other sensedata to the first individual, who is the "mind" considered. It seems doubtful whether a satisfactory answer can be given to this question so long as we continue to consider the body simply as a physical object. We shall now, however, proceed to give a more concrete meaning to the term "body" than it possesses when it refers (as it must do in the so-called "objective" sciences) to a certain class of sense-data.

It is not an easy matter to settle the exact ontological status of the objects presented to us in perceptual experience. In attempting to get beyond these immediate data, we must beware of employing the categories which we use in connection with events occurring within the object of experience. For while, if we consider the way in which knowledge has grown, we find that many of these categories in the fullest significance they have for us are applicable more widely than to the object of experience alone, this is not always the case. The category of causality, for example, as used in physical science, is strictly applicable only within the object of experience, although in the deeper meaning that we find it to possess for such individuals as ourselves if we investigate its basis as a concept (namely, efficient activity), it implies a reference beyond the immediate data of sense, the latter being 


\section{The Relation of Mind and Body}

in certain respects capable of interpretation in terms of it $^{1}$.

The matter we are now considering may perhaps be best approached indirectly. In the first place, it would appear that no entity which can be said to be existent in any really concrete sense of that term, can be an object of immediate acquaintance. For the essential nature of such an entity-the entity as it is in itself ${ }^{2}$ - could never be realized by any other entity, just as an individual like ourselves, for example, cannot know any other individual as he really is in himself. It follows that sense-data, being objects of immediate acquaintance, cannot strictly be regarded as existent. In particular, they are not existent in the sense in which the percipient subject is existent. But they have being of some kind - they are there, that is, they are presented to a subject.

Secondly, we must enquire whether sense-data, though not themselves existent entities, have their ground in existent entities. If we deny this to them we are forced to regard them as purely subjective modifications or states (in which case we might of course say that their ground was the subject itself and no other entity) and are logically carried on to solipsism. The latter position can no doubt be maintained in such a way as to be logically irrefutable. But it is to be rejected on grounds of a priori improbability and philosophical

${ }^{1}$ See the essay on "Scientific Method in Philosophy and the Foundations of Pluralism," Section VII.

${ }^{2}$ This is dealt with more fully elsewhere: ibid., Section V, and in "The Philosophical Problem raised by the Weber-Fechner Law," end of Section III. 
sterility. We must therefore postulate a ground of our sense-data, in existent entities other than ourselves.

Adopting this position, then, we infer that the existence of certain entities in certain relations to a subject, involves the perception by that subject of certain objects termed "sense-data," which may also be called the "appearance" of those entities to that subject, thereby defining the term "appearance." Hence we must so far regard appearance as an ultimate mode of being pertaining to the data of perception and to be carefully distinguished from existence, which is another ultimate mode of being. The existence of the entities referred to is manifested to us in the sense-data which make up the object of perceptual experience, and our interaction with them is manifested to us in the progressive differentiation of that object as experience advances. We are left with the question as to the nature of the entities concerned, and to the consideration of that we must now turn.

\section{The Pluralistic Interpretation of the Matter}

In rejecting solipsism, and postulating accordingly the existence of entities other than ourselves as the ground of our sense-data, we are faced at once with a possibility which is, to say the least, discouraging. It is that the essential nature of these other existents is utterly unlike that of anything about which we have knowledge. They may be quite unknown and unknowable to us.

Many philosophers have arrived at some such conclusion in the past. Herbert Spencer's "Unknowable," 


\section{I98 The Relation of Mind and Body}

and the "things-in-themselves" of Kant, are striking examples of this. And it must be admitted that there seems no way of disproving this alternative, at any rate a priori. On the other hand, we are by no means logically compelled to it provided we can justify empirically the other alternative of interpreting sense-data as the appearance of entities similar in nature to existents about which we have knowledge. Now each of us indubitably realizes the existence and nature of one such entity only-himself, and has knowledge about this entity contained in propositions based on that concrete realization. But we may proceed to assume that sense-data are the appearance of individuals fundamentally akin to us in that they are subjects of experience, and differing from us only in the indefinitely various levels of experience to which they have attained; and we may apply this hypothesis not only to the existence of other people, not even merely to the realm of organic matter in general, but to inorganic matter as well. It is in such an ontology that pluralistic spiritualism consists, and its justification must be looked for in empirical verification.

It is no part of our purpose here to advance the general arguments which support this hypothesis ${ }^{1}$. Suffice it to say that pluralism accounts satisfactorily not only for the behaviour and development of organisms in general from the amoeba up to man himself, but also for the apparent lack of spontaneity and the prevalence of fixed routine observable in the inorganic world. To pluralism we shall therefore have recourse as an hypo-

${ }^{1}$ See "Scientific Method in Philosophy and the Foundations of Pluralism." 
thesis which may help us towards the solution of the problem of body and mind, and the first step must lie in the restatement of the problem in terms of that hypothesis.

The object of experience, as actually given in perception, is an indivisible unity. But in any reflection on experience we have to regard this object as a whole analyzable into parts, the synthesis of which does in fact approximate very closely to the given indivisible unity. Now there must be an interpretation of the fact that this analysis, whose results approximate so closely to actuality, yields parts (sense-data) of various kinds and standing in various relations. There must be something corresponding to each kind of sense-data or group of sense-data-something which determines that analysis, though not entirely adequate in this case, should, so far as it goes, distinguish this particular kind and no other.

Among the sense-data presented to each one of us there is one group which is marked by the fact that there are always some of its elements given in perception. This complex is made up of certain sensations of sight and touch, together with organic sensations which can be correlated with them and which are said to make up what is called "general sensibility." We come to regard this complex as peculiarly our own, and, as we have seen, the concept of our own body is based on it. Not only does it differ from other groups of sense-data in the fact that some or other of its elements are invariably presented to us, but also in the fact that certain of its elements (organic sensations, for example) have a peculiar character which is quite unique. 
To the subjects of experience which constitute the realm of existent entities according to the pluralistic hypothesis, we may give the Leibnizian name of "monads." The facts we have just been considering can then be interpreted by supposing that among the monads whose appearance constitutes the object of experience of each of certain particular monads, there exists a group (perhaps even "society" may prove to be an appropriate term) which stands to the latter in a unique relation. The members of this group may be considered to have attained a level of experience inferior to that of the monad to whom they are related in this way. Moreover the members of the group are related to one another in at least some ways in which they are not related to other monads who are not members of the group.

An individual organism thus consists of a dominant monad in a certain relation to a group of subordinate monads. The former constitutes what is commonly called the "mind" of the organism. The latter, albeit themselves inferior "minds," constitute the "body" of the organism. In this way the concepts of body and mind can be interpreted in terms of existent entities as opposed to the appearances of such entities; and the determination of the relation between them consists in the determination of the relation between a dominant monad and its attendant group of subordinate monads. As will shortly be apparent, the necessary investigation involves an enquiry into the nature of certain subsidiary relations closely connected with the one in question.

We have thus at last given a more concrete significance to our problem-a significance of a type which is 


\section{Pluralistic Interpretation of the Matter $20 \mathrm{I}$}

probably at the back of most men's minds when they consider the relation of mind and body, though all would not agree as to the nature of the existent entities involved. Let us now see what can be said on the question when the nature of these entities is such as has been indicated.

\section{Application of Pluralism to the Solution of the Problem}

Before proceeding with our main theme, let us pause a moment to consider what type of result may be regarded as truly a solution of the problem we are examining. It may be remarked in the first place, that no statement of the relation of body and mind in terms of relations of the kind distinguished in the object of experience can be considered a satisfactory solution. For not only are propositions asserting the subsistence of such relations purely descriptive as opposed to explanatory, but they subsist in all cases between phenomenal objects, whereas we are here dealing with existent entities. In particular, the causal relation, as it is understood in physical science, is no exception to this. Empirical observation only warrants the assertion of the subsistence of causal laws among phenomena, i.e., general propositions whereby the occurrence of certain events can be inferred from the occurrence of certain other events. No such object, for example, as a "causal nexus," as it is sometimes called, which evidences the presence of some principle of efficiency, is presented in immediate experience. The existence of causal efficiency in the subjective interpretation is, 
of course, another and entirely different question'. But the point is, that no type of "action" observed in the phenomenal world can be applied to the case of the action of mind on body or of body on mind. To attempt such an application would bring us no whit nearer the solution we require.

On the other hand, it must not be forgotten that the pursuit of our enquiry will inevitably lead, sooner or later, to ultimate indefinables. Provided, however, the latter are such that their essential nature is fully realizable by us, the fact that it cannot be described explicitly with any adequacy matters not a jot. Indeed, the state of affairs which precludes such explicit description is the very goal to be sought after in all enquiries possessing any degree of finality. For if the result of enquiry be stated in terms of entities whose nature we clearly realize ${ }^{2}$, the mere indication of those entities provides a solution which can be called explanatory ${ }^{3}$ in the only true sense.

The matter which primarily concerns us is the relation of the dominant monad to the subordinate monads associated with it. Only second in importance to this, is the relation between the various subordinate monads which are members of one organism. Evidently a clear appreciation of the latter relation will depend upon

${ }^{1}$ See "Scientific Method in Philosophy and the Foundations of Pluralism," Section VII.

${ }^{2}$ See op.cit., Section V. Also Section VII, note on "activity." This "realization" is not the same thing as knowledge, which is a relation between two entities. We can only, of course, realize indirectly, as it were, the nature of any other entity, namely when this entity is essentially similar in certain respects to ourselves.

${ }^{3}$ Op. cit., Section III. 


\section{Application of Pluralism to the Problem 203}

a satisfactory determination of the nature of the former. But, in addition, there are three other relations which bear more or less closely on the question and which must not be passed over. They are : (I) the relation of monads which are members of the organism to monads which are not ; $(2)$ the relation, mediated ${ }^{1}$ by its subordinate monads, of the dominant monad to the monads which constitute the environment of the organism; and (3) the relation (if any) of the dominant monad to the environing monads, which is not so mediated. These relations must be briefly considered before the main issue, with a view to clarifying the latter more completely.

Consideration of the relation between monads which are members of one organism and monads which are not members of that organism brings us in sight of one of the limits of pluralism. For this relation must consist in efficient interaction of some kind between the monads concerned. Moreover, this interaction is of a relatively direct type. It is not mediated by an organic body, at least prima facie. But a mere plurality contains within itself no concrete ground of such interaction. Accordingly, we must here supplement pluralism by postulating as this ground some concrete all-pervading principle or entity which mediates the interaction of the monads, and which makes of their plurality a unity as well. With the exact nature of this entity, and of the relations in which it stands to the monads in particular and in general, we are not here concerned ${ }^{2}$. But it

1 As will appear in the sequel, "modified" is perhaps a more appropriate term to use in this connection.

2 For the theistic view see James Ward, The Realm of Ends, Part II, and especially Lects. XI and XII. 
should be pointed out that we must also postulate the entity in question as the ultimate ground of action and reaction between dominant and subordinate monads, and between subordinate monads of one organism, although the interactions here mediated differ in important respects (which it is our particular business to determine) from the interaction we have just been considering.

Of the relation, mediated by its subordinates, of a dominant monad to the environing monads we shall naturally be able to treat more fully when we have considered the main issue of the relation of dominant to subordinates. But it can at least be said here that the mediated relation of the dominant monad to the environment, although so mediated, is in some sense a direct relation. For what we perceive can certainly be legitimately described as the appearance of the environing monads, and not as that of the monads constituting our brain and sense-organs. On the other hand, it must certainly be granted that in general, though perhaps not invariably, the subsistence of this relation is conditioned by the association of the dominant monad with its subordinates. For while this association persists, the former cannot in general perceive the environment except through the mediation of the latter. The question as to whether such mediation is always necessary, leads to the consideration of the possible subsistence of the third type of relation, namely an unmediated $^{1}$ relation, of the dominant monad to the environment.

${ }^{1}$ That is, unmediated by the body. It is, of course, mediated by the universal ground we have postulated, but the body plays no part in that mediation. 


\section{Application of Pluralism to the Problem 205}

There are here three questions to answer: Is such a relation possible while the dominant monad is associated with its subordinates? Is it possible when this association no longer persists? Assuming the answer to either or both of these questions to be in the affirmative, is what is then perceived essentially similar in type to what is perceived in normal circumstances?

The first question is raised by the occurrence of such phenomena as clairvoyance and clairaudience. Two alternatives are here possible. Either a relation subsists between the dominant monad and the environment which is unmediated by the body, or else the relation is mediated by the body in some way, but the latter is thereby functioning abnormally. In any case, however, there seems no reason whatever for denying altogether the possibility of the subsistence of such an unmediated relation, even while the dominant monad is yet associated with its subordinates; and therefore a priori there is such a possibility when this association no longer exists. As to the last question, no definite answer can be given. But there is no reason to doubt that what is presented in such cases of "abnormal" perception, though differing in certain respects from what is normally presented, is not essentially unlike it in type ${ }^{1}$. In this connection, the fact that clairvoyance and clairaudience resemble normal perception in certain essential respects is not without significance.

Finally it must be remembered that, in the concrete,

1 We must, of course, admit a certain causal dependence, as regards their quality, of the objects of perception on the body; but this dependence is far from being such as to render objects normally perceived disparate in type from those "abnormally" perceived. 
the relations we have been considering consist in efficient action and reaction between the entities concerned, this interaction being manifested to the latter in perception ${ }^{1}$. With this reminder we may proceed to the main issue.

The human soul, when born into this life, appears at first to be remarkably helpless in dealing with the environment which surrounds it. Such control as it eventually gains would seem to be attained only through knowledgepainfullyacquired by experiment, or gradually imparted by other and maturer minds. At first blush then, the mind of the new-born is apparently indeed the tabula rasa that Locke supposed it to be. Further enquiry, however, shows that such a supposition is not really tenable. In the first place, we cannot assume that the individual has not existed prior to birth into this life. Pluralism carries with it the assumption of this pre-existence, and the further assumption that during the latter the individual was a conscious being. But though conscious, we may safely grant that the individual was not self-conscious. This he attains to only in his present life in the body. Consequently, although he may be said to have learnt a certain amount during previous experience, the results thus attained are not presented explicitly in his consciousness, for this is not possible till the level of conceptual thought is attained at which self-consciousness emerges ${ }^{2}$.

1 As previously noted, the whole presented to any subject in perception is the manifestation to him of the subjects with whom he interacts, and the differentiation of that whole is the manifestation of the interaction and its results.

${ }^{2}$ And then it only applies to what is experienced after this level is attained. 


\section{Application of Pluralism to the Problem 207}

But there is another and more important fact which shows that the individual does not enter this life entirely unequipped with means of profiting by the experiences of past ages. $\mathrm{Up}_{\mathrm{p}}$ to a certain point, and within certain limits, the development of the mind proceeds pari passu with that of the body. Now whatever may be said of the mind, it is certain that the body does not have to learn to grow ${ }^{1}$. The process by which the body of any given type of organism comes to maturity, is the same essentially whatever be its environment, provided the latter is such as to admit of its continued existence at all. And this relative independence of environment in the essential characteristics of development, is the more marked the higher we go in the scale of life, that is, the later the appearance of that type of organism in the evolutionary drama. Moreover, in growing, the particular body recapitulates the fundamental stages in the development of the species of which it is a member ${ }^{2}$. Such recapitulation has been regarded as due to a kind of "racial memory" of the monads involved". Whatever be the cause of it, it is a fact of great importance in the present connection.

In addition to this gradual growth, there is manifested at all stages of it certain habitual bodily reactions or reflexes which are partly, and apparently in many cases wholly, independent of the dominant consciousness. These are invariably teleological in nature (though not, of course, always of great practical use at this level of

1 This does not mean that the growth of the body is independent of any learning by experience, but that the latter occurred in the past.

2 See, e.g., Haeckel, The Riddle of the Universe, Chap. v.

3 J. Ward, op. cit., pp. 206-2 I 2. 
development), and serve to protect the organism as a whole, and to improve the situation in which it finds itself.

It is clear from the above, that the body may be looked upon as the storer-up of knowledge acquired in the course of past ages, and, in early life at all events, as the utilizer of this knowledge to the greatest advantage. As we have said, the experience hived in this way must be distinguished from the pre-natal experience of the dominant monad or "mind" of the organism. The presence of the latter store of implicit knowledge is perhaps manifested by the existence of those muchdiscussed "instincts" in which the dominant consciousness of the organism is primarily involved. Bodily reflexes, on the other hand, in which the dominant consciousness is not involved at all, or in which it is involved at most secondarily, may be regarded by analogy as due to the instinctive reactions of the subordinate monads.

We are thus brought in sight of one aspect of the relation between the dominant monad and its body. The latter may be regarded almost as the nurse or tutor of the former. The stored-up experience of the body is at the disposal of the mind to utilize as well as it can, and the method of utilization is itself almost wholly determined by the body in the earlier stages of the mind's development. And all this notwithstanding the fact that the dominant monad eventually attains to a higher level of experience than its subordinates; for it constantly happens in ordinary life that a person reaches a higher level than that of his former instructors. Thus we see the necessary actions in early life, while the mind is yet comparatively helpless, carried out almost entirely 


\section{Application of Pluralism to the Problem 209}

by the body. But as time progresses, the mind is brought through the body more and more successfully into touch with its environment. Moreover, in the course of this process the mind acquires a more and more complete control over the body itself. On the psychological side this corresponds to the explicit emergence of a true volitional factor - the replacement in large measure of non-voluntary by voluntary attention.

It is evident, then, that in this aspect the association of body and mind varies in degree of intimacy. The body is at first absolutely necessary to the successful introduction of the mind into the milieu in which it now has to play its part, and in the course of that introduction the association between the two becomes more and more intimate. But after a certain maximum is reached the presence of the body becomes in many respects a hindrance rather than an aid to the attainment by the mind of higher levels still, and the bond gradually loosens. This increasing, followed by decreasing, intimacy of association of body and mind is manifested on the side of the former by its inevitable growth, maturity, and decay; and when at last the bond is broken altogether, the body dies because the mind is no longer associated with it and not vice versa.

One more point here. The body serves another important purpose in that it makes possible the formation of certain fundamental concepts by the mind, whereby the latter attains self-consciousness ${ }^{1}$. It is a commonplace that the existence of the body is an allimportant factor in the development of self-consciousness. More will be said on this later, but if further

${ }^{1}$ See, e.g., J. Ward, Psychological Principles, pp. $364 \mathrm{ff}$.

R. S. P. 
evidence were needed of the function of the body in bringing its dominant monad into harmonious interaction with the environment and of enabling it to develop highly, it is undoubtedly to be found here.

Let us now consider for a moment the effect on the organism of what is called a "physical stimulus," i.e., an action originating in something which is not a part of the organism. The chief point to notice is that in very many (perhaps most) cases, the organism reacts as a whole. Particular parts of the body may appear to be affected more than others in the course of this action and reaction, but notwithstanding this, an adjustment takes place which involves the whole organism. Nor is the reason for this far to seek. It is teleological. The reaction of the organism refers to a purpose which has as its object the well-being of the organism as a whole in the changed situation brought about by the application of the stimulus; and in general this reference to the whole involves a particular direction in so far as the dominant consciousness enters into that whole.

Not only is this reaction as a whole a fact when the stimulus is something such as a light-impulse, carrying no immediate reference beyond itself; it is still more marked when the stimulus is, for example, a spoken word, that is an object invested with meaning and thereby carrying an immediate reference to other objects. And in this case, the particular rôle of the dominant consciousness which involves the consequence that the reaction shall be a reaction of the organism as a whole, is especially evident. In reducing the relation of body and mind to its ultimate form, we must therefore take 


\section{Application of Pluralism to the Problem $2 \mathrm{I} \mathrm{I}$}

full account of that unity of the organism which the above facts so clearly demonstrate.

We are now approaching very closely the heart of the whole matter. Let us turn back for a moment and consider the processes observed by the physiologist which are the concomitants of sensation. So far as the physiologist can tell, the sense-organ is stimulated from without and in consequence an impulse is transmitted along the nerve-fibres which eventually reaches and affects a sensory centre. At this point the physiologist is generally content to leave the matter, simply remarking that in some extraordinary way the impulse "passes over" from the sensory centre and gives rise to a sensation. Thus, from the purely physiological point of view, the sensation-process is analogous to the handing in of a message at a telegraph office (the sense-organ), its transmission over the wires (nervefibres) to a receiving station (sensory centre), followed by its delivery to the addressee (the percipient subject). But this cannot possibly represent the process as it actually is; for there is nothing in any way corresponding to this in the nature of sensation as it is realized in experience by the sentient individual concerned. Rather may the latter almost be said to "identify" himself with the bodily apparatus in question. We cannot say, for example, simply that the eye sees, or even that the whole visual apparatus sees, including the nerves and sensory centre. It is the subject who sees with the visual apparatus. Nor is this way of looking at it vitiated by the fact that the nerve-impulse takes time to travel from the periphery to the sensory centre; for this simply means that sensation is a pro- 
cess. But we must regard this process as a whole, including the temporal factor. We have here, in fact, what Dr James Ward' has described as a "functional" relation of the subordinate monads to their dominant monad. This statement, however, calls for reservations. It must not be implied that the mind uses the body simply as a man uses a tool, which is an object quite apart from him and in which, even when using it, he is in no sense present. We cannot set the mind over against the body in this way, as merely transcending it. We must say rather that the mind perceives with the body in so far as it is immanent in it.

Mutatis mutandis, we find the same in the case of volition. Here physiology traces the impulse from the higher centres to the motor centres and thence to the muscles. From this point of view, the process appears as the issuing of a mandate by the subject, which is mysteriously communicated to the brain and hence transmitted to the appropriate parts of the periphery. But in the actual volitional experience there is nothing remotely resembling this. Again the dominant monad is a factor whose presence is inherent in the process as a whole. The former does not stand at one end of a chain of which the movement willed is the other.

To one who has followed the argument so far, it will be evident that we are here struggling to express something which by its very nature is incapable of adequate expression. Thus we arrive at last at the ultimately indefinable essence of the relation between body and mind. That relation consists fundamentally in what we have called the "immanence" of the ${ }^{1}$ See The Realm of Ends, Supplementary Note III, pp. 46 I ff. 
dominant monad in the subordinate monads. This immanence is strictly indefinable, and what we have been endeavouring to elucidate are really consequences and partial expressions of it. Yet although we cannot define it, we can realize what it is in its actuality. Especially is this the case, as we have seen, in sensation and volition; and the point to be insisted on is that the conception of the relation of body and mind based on this realization (so far as we can conceive it at all) must not consist in picturing the body as a line or network of communications, at one end of which stands the mind or dominant monad, and at the other external reality. Instead we realize the organism in interaction with the environment to be a unity comprising a dominant monad immanent in what (following pluralism) we regard as a society of subordinate monads. This profoundly intimate association is almost as fundamental a realization as that of our own existence. But, on the other hand, we must not lose sight of the fact that in another aspect the mind transcends the body. If this were not so the body could not, for instance, ever be object for the percipient subject associated with it. The relation of this immanence and transcendence, and the extent of each, it will be necessary to consider later.

The immanence we have been considering may be compared with the "sympathetic rapport" mentioned in this connection by Dr James Ward". This rapport is conceived as akin to telepathy - a relation of subject

1 Op. cit., p. 463. The phrase was first used by Lotze, Metaphysik, Bk. III, Chap. v, Sec. 300 . The kind of rapport thus imagined seems far more conceivable as based on immanent action, than as based on any kind of transeunt action. 


\section{I 4 The Relation of Mind and Body}

and subject, in which neither appears to the other as object. We have preferred to use the term "immanence," however, and to reserve for "rapport" a wider meaning, as involving any relation between two subjects whereby each affects the other. Pluralism then implies the existence of a rapport of some kind and degree between every subject in the universe and every other subject, and in general this rapport is manifested to each of the subjects concerned by the appearance or presentation of an object.

Although we are not ordinarily conscious of such processes, for example, as digestion or the beating of the heart, as we are conscious of such processes as sensation and volition, it does not follow that the mind is not immanent in the whole body. For, in the first place, on our theory that the body dies because the mind is no longer immanent in it $^{1}$, we have an obvious interpretation of the fact that at death digestion and the beating of the heart cease ; namely that the mind which was formerly immanent in these processes is no longer so. And secondly, we have the occurrence of organic pain, which indicates the immanent relation of the mind to the whole body, and the effect on the former, in consequence of that relation, of abnormal (and therefore in general harmful) functioning by any member of the latter.

Viewed more from the bodily side, we may look upon the immanence of the mind or dominant monad as the concrete principle in virtue of which the subordinate monads composing the body are co-operant. For it is to be noted that the actions of these subordinate monads, although in one aspect they may be

${ }^{1}$ See p. 209 above. 


\section{Application of Pluralism to the Problem 215}

directed to the conservation and betterment of the agents concerned not only as individuals but also as a society (the body), are also directed with reference to the ends of the organism as a whole, controlled as that organism is by the immanent dominant monad. In fact it is in this important respect that the organism differs from a human society. For the members of the latter act for their own ends and for those of the whole society, but, so far as we can see, there is no further individual embodied, as it were, in the society, to whose purposes their actions also have a reference ${ }^{1}$. This unique factor in the behaviour of an organism is but one more mark of the presence of a mind immanent in the body of the organism as a whole. And on the other hand, we can see how, in virtue of their intimate association with, and dependence on, a single supreme controller, the monads composing a body are limited as to the extent and nature of their self-development as individuals, in a way in which the monads composing a human society are not limited.

We have emphasized the fact that, in general, the organism reacts to a stimulus as a whole. This remark applies most forcibly to the most highly developed organisms with which we areacquainted, namely human beings. At the same time, however low we go in the scale of life, we find organic reactions to be determined largely with reference to the interests of the whole organism. But, on the other hand, there is apparent in the lowlier

'A human society is sometimes described as itself being "overindividual," but this does not imply the existence of an additional individual superior to the members of the society See, e.g., J. Ward, op. cit., pp. $129 \mathrm{ff}$. 
organisms a measure of independence in the parts which in some cases reaches such an extreme that the absolute separation of the organism into two or more portions is followed by the continued independent existence of those portions. In some cases each portion re-develops into a replica of the original organism, in some cases not. In such comparatively loosely aggregated societies, it seems reasonable to postulate the existence not of a single dominant monad, but rather of a plural monarchy of a number of monads of more or less equal rank, though not always necessarily fulfilling the same functions. We have the counterpart of this in the higher organisms, culminating in man. For although there is here a single dominant monad immanent in the whole, yet the functions of the body exhibit a certain degree of decentralization, i.e., certain functions are relatively independent in themselves, though all the functions are co-ordinated with a view to ensuring the well-being of the whole. Thus we have grounds for assuming the existence of a certain number of sub-dominant monads with their associated retinues of inferiors. In this way the organism may be regarded as a group comprising sub-groups each of which is controlled by a sub-dominant monad, while the single dominant monad is immanent in the group as a whole, as the ground of that co-operation of the members which is apparently directed ultimately with reference to the interests of a single individual ${ }^{\text {. }}$.

Such considerations more or less define the relations of the subordinate monads to one another, which

${ }^{1}$ This conception of a hierarchy of monads in the body originated with Leibniz. 
we previously referred to as subsidiary to our main problem. These relations are of course fundamentally constituted by the immanence of the dominant monad in the whole society ${ }^{1}$. But it is to be noticed, as we remarked before, that in early life the dependence is mainly one of mind on body, whereas this state of affairs is gradually reversed as time goes on, the mind gaining a more and more complete control over the body. Later the bond again loosens, as witness the lack of control of bodily functions and expressions manifested in senility, to be finally dissolved in death. Hence it would appear that at first the organism is mainly controlled by the sub-dominant monads and their inferiors in virtue of their stored-up experience of the racial past, while these quondam monarchs themselves eventually pass more and more under the control of the dominant monad. We must therefore suppose that that association to which we have given the name of "immanence," and of which this control is the very sign and symbol, admits of degrees of intimacy. And, finally, it may be remarked in this connection that in cases where we may fairly suppose this immanence to have ceased abnormally, as, for example, in paralysis, the helplessness of the dominant monad to control the part affected is accompanied in general by the atrophy of that part. This is additional evidence that the rela-

1 It may be regarded as probable that the objects presented in perception to the subordinate monads consist, at least in part, of the appearances to them of other monads associated with them as members of the same body. Others of these objects may, and in certain cases apparently must, be the appearances of monads forming part of the environment. 
tion of the subordinate monads is constituted essentially by the immanence in them of the dominant monad.

Let us now turn back for a moment and reconsider the three types of relation which we previously examined as subsidiary to the main issue, in the light of the results we have so far arrived at. Stress was laid on the fact that the relation of the dominant monad to the environment, mediated though it be in some sort by the body, is yet a direct relation. The reason of this is now clear. For we have seen that the body must not be considered to stand definitely between the mind and the environment as a communicator from one to the other. Instead we have the organism, consisting in the mind immanent in the body, interacting as a whole with the environment. From the point of view of the other persons who constitute part of the environment of an individual, we may accordingly consider what they perceive as manifesting the existence of that individual, to be the appearance not merely of his body, but of the whole organism constituted by himself immanent in his body. This is the easier to realize when we remember how the hundred-and-one tricks of expression and gesture, trivial though they usually are individually, manifest continuously the character of the person concerned.

The second type of relation, that of the body to the environment, evidently merges into the first type. For, as we have seen, in interaction (at any rate normal interaction) with the environment, we have not two entities acting separately, as it were, or as independent links of a chain, but a single whole, the organismmind immanent in body-interacting as a whole with the environment. It may be repeated, however, that this 
interaction must be mediated by that concrete entity, whatever it may be, which is universally immanent in all interacting entities ${ }^{1}$.

We are left to consider whether the dominant monad, in so far as it transcends the body, is capable of interaction with the environment independently of the body. While the immanent relation also holds, such interaction is prima facie improbable. There may be abnormal perception, it is true, but this is perhaps accompanied by bodily process which differs in some important respects from the process involved in normal perception; just as the objects of abnormal perception differ in certain typical respects (lack of tangibility, for example) from those of normal perception. We have this typical difference in cases of abnormal perception, when, for instance, the nature of the associated abnormal bodily process can be observed or immediately inferred. The internal stimulation of a sensory centre, independently of the corresponding sense-organ, may be quoted in illustration, in which case there arises one kind of hallucination.

On the other hand, there is nothing in all that has been said which might controvert the possibility of any interaction of the dominant monad with the environment except as immanent in the body. In other words, we have no reason so far to suppose that when this immanence ceases at death, the interaction of the mind with other minds ceases also, and perception and volition with it. No doubt the objects of perception and the results of volition in bodily life, are qualitatively modified to a certain extent by the association of the 
mind with the body ${ }^{1}$; but it only follows from this that when the association no longer exists, the objects of perception and the results of volition differ qualitatively to some extent from their former nature, not that they cease altogether, not even that there is absolute qualitative discontinuity. But the possibility of this continued existence of the dominant monad after bodily death, brings us face to face with certain factors of decisive importance, not only in our present problem, but also as evidence for or against that possibility. We refer to the connection of memory and imagination with the body. To the consideration of this topic we shall now proceed.

\section{Memory and Imagination and their \\ Dependence on the Body}

The possibility that memory and imagination depend necessarily upon the body, depend, in other words, for their continued existence upon the permanence of certain physical traces in the brain which constitute an objective record of the past, is one which, if actual, would be fraught with consequences of the gravest moment for every human being. For it would mean that with the decay of those physical records following upon death, all possible connection of the individual mind with its past history would have ceased. The further existence of that mind would, in view of such an absolute breach of continuity, be personally valueless. In fact, there would no longer be a person, as such, but

1 Similarly we may suppose the perceptions of the subordinate monads of each other and of monads "external " to the body, to be modified qualitatively by the immanence of the dominant monad. 
rather what in all essential respects would be equivalent to an individual who was beginning his existence and development wholly $a b$ initio. Strictly speaking, it would not be the future life of the same individual, at least in any sense to which we might attach value and importance, but the life of what would to all intents and purposes be another individual ${ }^{1}$.

But we cannot decide as to the dependence of memory and imagination on the body until we have come to some conclusion as to what the nature of these processes really is, as distinct from their manifestation in experience. And before considering the specific differences between imagination in general and memory in particular, we must try to decide what is manifested by the images, as opposed to impressions, which are concerned in both.

In the first place, it is evident that there must be an important connection between impressions and the corresponding images, while on the other hand, there are manifest differences which are just as important. The latter fall essentially into two types. Firstly, there is a difference in what has been termed ${ }^{2}$ (but not very satisfactorily) "vividness" - a difference most difficult to put into words though everyone is quite clearly aware of its nature. Not only are images less clear in general than impressions ; they also lack the "bite" or "tang," as it

1 It is sometimes held, however, that the nature of the individual would be modified by his former existence, though, as far as he was concerned, he would be starting life altogether afresh, and quite unconscious of this modification. See McTaggart, Human Immortality and Pre-existence, pp. $98 \mathrm{ff}$.

2 By Hume. 
were, of the latter. Secondly, images lack those marks, so characteristic of impressions, whereby we intuit the reality of entities other than ourselves ${ }^{1}$. In particular (and most notably) among these marks, we have tangibility, fixity, and local sign ${ }^{2}$.

In spite of these differences, however, there is a sense in which images may definitely be said to resemble the impressions corresponding to them. Apart from such resemblance we should of course have no ground for judging that a particular image corresponded to a particular impression. The similarity occurs in respect of such qualities as shape, colour, sound, taste and smell; though the two latter are usually very difficult to revive ideally.

In any hypothesis as to the nature of images, we have, then, to account for the above facts. But in addition to the latter there are two other facts which are very suggestive in helping us to frame such an hypothesis. When a presentation recurs in experience, sometimes even one to which we did not previously attend with any particular interest, there is a subtle characteristic attaching to the second experience which was absent in the case of the first. We say that the impression is now "familiar" to us-we recognize it, though not always explicitly. Now it is evident that this familiarity cannot be something intrinsic in the impression alone. On the objective side there is no reason why this additional mark should be attached to the impression the second time and not the first. In other words, there is a subjective factor in the experience

${ }^{1}$ See J. Ward, Psychological Principles, Ch. vi, §6.

2 J. Ward, op. cit., p. 147 . 
of familiarity ${ }^{1}$, and this must be the essential factor. For although the second impression thereby differs somewhat from the first (owing to what has been called "assimilation" ${ }^{2}$ "), the ground of the difference is subjective.

Secondly, there is the fact of association; that is, previous impressions tend to be revived ideally in the order in which they were originally attended to ${ }^{3}$. Now we cannot explain this association in any intelligible way simply by considering the images as such. Attempts at an explanation of this kind have been made by assuming the existence of a mysterious cohesion or attraction between the images. No satisfactory result has thereby been attained, however, for there is no reason to be seen why these forces occur between just such a set of images and no other, if we look merely to the images themselves. It is, therefore, necessary to consider instead the part played by the subject in the matter, and this necessity is emphasized by the fact that the order of revival is determined by the order in which the original impressions were passed under review by that essentially subjective function, attention.

In view of the foregoing, we may adopt an hypothesis provisionally ${ }^{4}$ somewhat as follows: In the first place, it must be supposed that subjective activity is characterized

${ }^{1}$ See, e.g., J. Ward, op. cit., p. I8o.

2 Ibid., p. 82.

${ }^{3}$ Ibid., Chap. viI, § 3.

${ }^{4}$ Our line of thought must now inevitably become speculative to a very large extent. Yet the course of speculation is governed and corrected by the facts we have been considering, and the results we shall arrive at are justified so far as they give an adequate interpretation of those facts. But it may be freely admitted that there is much room for discussion here. 


\section{The Relation of Mind and Body}

by a certain functional inertia. When it has at any time taken a certain "direction," and is subsequently started along the same line again, it tends to carry on, as it were, to the same end as before. This, however, will not take us very far until we have also considered matters on the objective side, that is, have decided what is the real nature of the processes manifested by impressions and images respectively.

First of all as regards the differences between impressions and images, we may certainly postulate that in the presentation of the former there are entities concerned distinct from the percipient, whereas in imagination this may not be so. At any rate, the same entities are not concerned in the latter as in the former, though we must suppose the effects of their interaction with the subject to linger on, thus accounting for the resemblance between images and the corresponding impressions.

This brings us to the second part of our hypothesis. Let us grant that the activity of the subject, though perhaps essentially of one type only ${ }^{3}$, is potentially capable of determination in indefinitely various ways. This is manifested in experience by the differences in quality and type of presented objects ${ }^{2}$. Now the nature of any particular determination will depend not only on the subject, but also on the other subjects with whom he is

1 This is, for us, in experience, attention.

${ }^{2}$ These, in sense-perception, we have termed the "appearances" of other entities to the subject. Evidently the quality of any one of them depends on the nature of the particular entity or entities concerned, and therefore on the particular way in which, by the interaction of the latter with the subject, the part played in that interaction by the activity of the subject is determined. 
interacting. It is the actual occurrence of this process of determination through interaction with others which is manifested to the subject in the perception of impressions. In other words, what is really occurring in such a case is the limitation or determination of the subject's activity in a particular way by the activity of other subjects. This process is manifested to the subject by the presentation of a certain type of object which we term "impression." And it must be noted particularly that the latter is the manifestation of the process of determination while this process is actually occurring. Thus it is evidently identical neither with the subject that perceives it nor with the other subjects with whom he is interacting, though its being is dependent upon both. Moreover, its mode of being is unique. It is, for example, distinct from the "existence" of the subjects-all of which we have previously commented on, when considering sense-data from another point of view ${ }^{1}$.

It would thus appear that the activity of the subject when directed in any particular way, takes on a certain "form," as it were, the nature of which is manifested by the corresponding presented object and determined by the nature of the particular entities with which the subject is interacting by reason of this particular direction of his activity. Owing to the functional inertia we have postulated with regard to the latter, it maintains this form to a greater or less degree of precision when it is for any reason directed the same way in the future. This maintenance of form, when the conditions which originally determined it are no longer present, is mani-

1 See above, pp. $195 \mathrm{ff}$.

R. S. P. 
fested to the subject by the presentation of the image of the original impression.

It is necessary at this point to avoid a possible misunderstanding. The better so to do, let us be quite clear as to the difference between the two processes manifested by the presentations of an impression and an image respectively. In the case of the first the determination of the activity of the subject to a particular form by the action of other entities is actually in course of occurring, and it is this process as actually occurring which is manifested to the subject by the presentation of a sense-impression. Hence our theory by no means compels us to regard the latter merely as a subjective modification. It is, as we have previously pointed out, distinct from the subject as such, though dependent in part on the subject for its being. In imagination, however, the presented object manifests a determination of the subject's activity which occurred in the past. When the activity is directed in this way, the determination of its form is already an accomplished fact and not a process which is now taking place, and the original agents to which it is due are no longer present ${ }^{1}$. Hence on this view we must undoubtedly grant that images are subjective in a sense in which impressions are not. But surely this conclusion is warranted by experience.

Let us take an analogy (which, however, must not be pressed toofar) by borrowing Locke's illustration from the tablet of wax. The latter represents the individual mind. Suppose an impression is made upon a certain part of it by a seal. The seal represents the external

${ }^{1}$ Strictly the term "present" can only be applied to an object, its use here being metaphorical. 
agent, and it is to be noted (which Locke failed to do) that the impression is a joint product depending on the nature of the wax as well as of the seal. Now the objects of sense-experience manifest the occurrence of a process which corresponds to the actual impressing of the wax by the seal ; but in future, when the subject's activity is directed a certain way (corresponding to that particular part of the surface of the wax), the presented image manifests something which corresponds to the wax as already impressed, though the seal is no longer present. Moreover, another point of analogy is that the impression on the wax will not remain clearly defined if the latter has meanwhile been affected by other actions. In time, it may even disappear altogether as regards any form recognizable as resembling the original.

By this hypothesis we may thus account for the resemblance in respect of certain qualities between images and the corresponding impressions, and also for their differences, not only in respect of "vividness," but also in respect of those marks (present in impressions, absent in images) which we take as manifestations of an existing "external" reality. Further, the subjective factor in familiarity is now satisfactorily explained. For a.recurring impression corresponds to a re-determination of the subject's activity in a form in which it is already determined as the result of a previous interaction. Again, it is now apparent why in a series of impressions $A B C D \ldots$ the revival of (say) $B$ tends to call up $C$ but not $A$. For the functional inertia of activity tends to arry it on its previous course once it is started in the iame direction by an occurrence manifested by a second oresentation of $B$ (or its image $b$ ), and the form it takes 
in this direction having previously been determined by the interaction which was manifested by the presentation of $C D \ldots$ it now takes the same form again, and as a consequence the images $c d \ldots$ are presented. Takes the same form, we say, but with the reservation that this form falls off in varying degrees from the clearness of its original definition. For the activity of the subject does not, strictly speaking, consist in a number of discrete acts, but is continuously directed in indefinitely many successive ways, each of which continuously modifies every other. In this we have the reason for the varying clearness of definition of memory-images, and their frequent evanescence.

But how is activity set going in any particular direction? That is, in what circumstances are particular images called up? A train of images may be called up by the presentation of an impression ${ }^{1}$ which was formerly followed by the impressions of which they are images; or it may be called up by the image of that impression; or, finally, in consequence of the doubling and re-doubling of the memory-continuum ${ }^{2}$, it may be called up by any object previously associated with it-by a concept, for instance. In all cases, however, the actual fact is the tendency of the subject's activity to retrace its previous steps owing to its functional inertia, although any particular tendency may be indefinitely modified in course of time, even to the point of practical disappear-

${ }^{1}$ Activity is, of course, initiated subjectively for the most partin experience, we say that the subject attends to some particular object. But, once initiated in any direction, it tends to take the same course as when previously set going in that direction, and hence to be determined in the same way.

${ }^{2}$ See James Ward, op. cit., Chap. vil, § 4 . 
ance, by the development of other tendencies as the result of fresh interactions.

Before coming to the question of the dependence of memory and imagination on the body, we may pause a moment to state very briefly the specific differences between those two processes. Psychological analysis shows ${ }^{1}$ that the presentations involved in memory differ from those involved in imagination in general by the possession of a greater fixity, more circumstantiality, and definite temporal signs in virtue of which they are localized in the past in a fixed order. The general ideational continuum is formed in the first place by continued reduplication, or bending back upon itself, of the memory-continuum, in the course of which the images gradually lose their concreteness (becoming more "generic") and temporal signs, while with the disappearance of the latter their order is no longer fixed. In both cases, however, the images involved are essentially the same in nature, and the hypothesis above advanced to account for these images will apply whether we are dealing with memory proper or with imagination. In the former the tendencies are more specialized, functional inertia leading to the revival of images in the original order of the corresponding impressions. In the latter the tendencies have, in course of time, become more generalized by continuous and varying activity, which process is manifested by the formation of the ideational from the memory-continuum. At the level at which this occurs, concepts also are formed, and become associated with the corresponding images. In consequence of functional inertia, when attention is directed ${ }^{1}$ See James Ward, op. cit., Chap. virI, § r. 
to a particular concept the corresponding image also tends to be presented; but, except in this respect, this inertia plays little part in thinking or in reverie, for the activity of the subject is here for the most part striking out entirely fresh paths.

On the basis of the theory we have been considering, a definite answer may now be given to the question of the dependence of memory and imagination on the body. Inasmuch as the mind or dominant monad is immanent in the body, the nature of the determination of its activity by other entities is modified by the body. That is, the nature of an impression, and therefore the nature of the corresponding image, depend partly on the body. But once the activity of the subject is thus determined, there is no reason why its future repetition as determined (manifested by the presentation of the image) should be necessarily dependent on the continued existence of the body. On the other hand, in virtue of the immanence of the mind in the body, it is probable that such subjective activity is accompanied by bodily concomitants, the latter perhaps being inevitable while the body continues to exist. In other words, bodily injury or abnormal functioning through any cause, may render certain directions, and the corresponding determinations, of subjective activity impossible; though the latter may again become possible, not only through bodily recovery, but also through the dissolution of the bond between body and mind by death. In bodily life the proper functioning of the body may be necessary for the formation of images, though not for their form, once the latter is determined; but it does not follow that the formation of images is impossible without the body. 
A man compelled by physical constraints to move in certain ways (whatever external forces be applied) cannot perform even those movements if a block occurs, but he regains the power of these and many other movements when once the whole constraining apparatus is removed. Nor is our conclusion modified in any way by the fact that certain presentations (as in some types of hallucination or of internal stimulation of sensory centres) are due entirely to bodily occurrences. For it is still the activity of the subject which is being determined, this time by the action of the subordinate monads composing his body alone, and the activity as thus determined can be repeated (for we can remember an hallucination) with the body, or without it if the dominant monad is no longer immanent in it.

Whatever we may think, then, of the dependence of memory and imagination on the body during bodily life, we have no reason at all to suppose that they cannot continue after death. They may even be enhanced. As for the associated images, so far at least as they refer to what was experienced during bodily life, they would manifest a continuity of type in all essentials. Can the subject therefore exist without getting another body, in virtue of the level to which he has attained during this bodily life? or must he, in default of such a new acquisition, fall back into the abysmal depths of experience at its lowest level? We cannot decide without referring to the nature of the personality to which we attain in this life; and to that we shall now turn as our final topic. 


\section{Vi. Personality}

We saw that the function of the body in early life is to mitigate the initial helplessness of the mind in its entirely novel surroundings by means of the stored-up experience of past ages, and thus to bring the mind gradually into a sure touch with those surroundings. But the part played by the body in the development of experience comprises more than this; and in its additional rôle it figures objectively rather than subjectively. On the perception of the body is based the growth of those concepts by the development of which the individual attains to the level of self-consciousness.

The relative permanence of that group of sensations which makes up the body as object, is the initial ground of our awareness of our own persistence as individuals. Accordingly the earliest form of the concept of self is that of the "body-self ${ }^{1}$ " The man identifies himself with his body, and it is only at a much later stage of experience that he is aware of himself as an individual distinct from, and to that extent transcending, his body. The perception of other relatively fixed groups yields eventually the concept of other individuals, their bodies being distinguished from our own in the first instance by such contrasted sensations as those of double and single touch. In so far as these other bodies appear and act in ways similar to our own, we refer them to other individuals of a nature akin to ourselves. In this way social concepts and the concept of self emerge pari passu. But the main point is that but for the existence of our bodies, we should never, from the level of experi-

${ }^{1}$ See James Ward, op. cit., p. 365 . 
ence at which we start, be able to attain to that higher level where we have become rational, self-conscious beings.

As was implied throughout our consideration of memory and imagination, the growth of experience, manifested on the objective side by progressive differentiation and redintegration of a presented whole, consists on the subjective side in the development of tendencies to act in various ways. The tendencies developed in this way must by no means be regarded as severally specialized and absolutely distinct from one another. Each modifies, and is modified by, all the others. The active subject is essentially a unity.

At its highest level, subjective activity takes the form of intellection. Speaking metaphorically we become equipped, as experience advances, with a conceptual "apparatus" which enables us to deal more or less successfully with present situations and helps us to cope with any fresh situations that may arise, even if the latter are entirely novel. For supposing this novelty to be a fact, we at least know how to set to work in our practical investigation of the case in order to obtain most successfully the requisite knowledge whereby we may adapt ourselves to the new circumstances. We have evolved, in the course of time, definite methods of scientific procedure calculated to yield the data required with a maximum of accuracy and a minimum of fruitless endeavour.

Not only is the existence of the body a necessary condition of the attainment of rational self-consciousness, but, as we have seen, the particular determination of activity, at all levels, manifested by the particular nature 
(as distinct from type ${ }^{1}$ ) of the presented object, is modified in process of occurrence by the body. It is also true, however, that once determined, activity is not necessarily dependent for its possibility on the existence of the body, though while the subject is yet immanent in the body, the possibility of his activity may be linked with the possibility of the occurrence of the associated physiological concomitants. But the fact that a high level of activity, though dependent for its attainment on the existence of the body, can, once attained, dispense with the body, leads us to regard our life in the latter as essentially a process whereby we get for ourselves a soul, if it may so be put; whereby, that is, we come to the explicit realization of our own existence as individuals, and so achieve personality.

In this sense, and in this sense only, can we legitimately make use of the much-abused phrase "content of self." The growth and enrichment of this content is manifested to the subject concerned by the continued development of the conceptual and ideational "tissue," as it has been called". But it must not be supposed for a moment that there is anything fundamentally distinct from the subject, and consisting in, or corresponding to, his images and concepts, which he drags about with him in an ever-increasing load, albeit for the most part implicit in sub-consciousness ${ }^{3}$. Such a view gives to the strictly metaphorical phrase "content

1 By "type" is here meant such a class as "image" or "impression" or "movement," etc.

2 By James Ward.

3 That is, so far as the images and concepts themselves are concerned. 
of self" a literal and wholly illegitimate interpretation to which it would be difficult to assign any exact meaning.

In the case of any individual, as distinct from other individuals, what we have actually is an active subject whose activity exhibits continually developing tendencies whose form is determined by interaction with other subjects. Images presented above the threshold of consciousness manifest to the subject the issue of such a tendency in actuality in a form largely determined by some previous sense-impression due to interaction with others. Subliminal images, on the other hand, are the manifestation of the existence of tendencies in subjective activity which at themoment are not realized in actuality ${ }^{1}$. The tendency to act in a certain way may exist though the subject is not acting in that way. Thus although the subject is active both in sense-perception and in imagination and intellection, in the case of the former he is interacting with other individuals like himself, whereas in the case of the two latter he is not ${ }^{2}$. Thus imagination and intellection are subjective in an absolute sense in which perception is not.

It is in this way that the developing active self comes to transcend the body, not only so far as the body is objective for him, but also by the gradual acquisition of the power of acting (and acting successfully)

${ }^{1}$ See next essay, Section III, for a detailed consideration of this.

2 As pointed out, in virtue of the immanence of mind in body, imagination may necessarily involve bodily concomitants so long as that immanence persists. But imagination must not be regarded as manifesting interaction between mind and body in any sense analogous to that in which sensation manifests interaction between the mind and the environment. 
independently of the body. And, be it noted, this transcendence is not gained at one bound, but is gradually achieved, and achieved while the subject is yet immanent in his body. That very immanence now restrains the acquired potentiality of independent activity and the body is no longer a useful instrument, but an irksome burden, a burden whose weight seems to increase, till death at last brings release and opens up a vista of new and loftier possibilities.

In this way we may interpret the drama of life as youth gives way to maturity, maturity to old age. The child-mind, following its sudden entry on the stage, gropes its way by means of the body in which it is immanent to an understanding of its environment, acquiring in the process a more and more complete control of its body and a more and more intimate association therewith. Yet the capabilities of the body are but limited, and anon the mind in thought far transcends it. Straining at the leash, it tires awhile; its quondam associate, now that the bond of mutual co-operation has lost its full harmony, passing rapidly to decay. Let us then dare to look on Death as a deliverer, and not a destroyer, who revives the weary and opens the way to a greater happiness in the exercise of those higher powers so painfully acquired in the body, so painfully restrained by it erstwhile.

It remains but to add a few words as to the nature of that future life to the belief in which the consideration of the relation of body and mind has finally led us ${ }^{1}$. To many it seems inconceivable apart from a

${ }^{1}$ There is, of course, no logical necessity in virtue of which the fact of a future life follows from the facts considered. Enough if we 
body of some kind. Are we again to become mere naked monads, it is asked? Perhaps, but if so how different should we be from those primitive undeveloped entities which figure at the bottom of the scale. They cannot hope to rise till they obtain a body. But we, who live in the body, pass beyond and above it at death. Admittedly it is a hard question to answer, and it is no part of our purpose here to embark upon the attempt. Yet we may say that in view of the results we have reached, there seems no reason to suppose that beings who have reached the rational self-conscious level depend for the continuance of their existence at that level upon the acquisition of another body. For they have acquired something of far higher worth-a personality, and all that it implies. Yet again we may admit that further consideration might lead to another conclusion. Our enquiry has carried us beyond the original problem and left us with one more unanswered question; but as the solution of the former does not depend upon the answer to the latter, we may leave our investigation at this point, and conclude with a brief recapitulation of results.

\section{Summary and Conclusion}

At the outset of our investigation, we found that the source of many of the difficulties associated in the past with the problem of body and mind, lay in the fact that most thinkers had approached that problem, directly or indirectly, from the standpoint of the Cartesian have shown the possibility of an existence beyond this body, an existence which other considerations, perhaps, not dealt with here, render highly probable. 


\section{The Relation of Mind and Body}

dualism. The inherent artificiality of this standpoint led to the prolonged discussion which has come to its logical conclusion in the methodological principle of psychophysical parallelism, the adoption of which is a confession of metaphysical failure. The only hope of changing this state of affairs lay in making an entirely fresh start from the only point of view possible if artificiality is to be avoided, namely that at which we are placed in actual fact-the duality of subject and object comprised in the unity of experience.

It then appeared that the "material bodies" with which physics deals, consist in certain groups or classes of sense-data ${ }^{1}$. The body, looked upon as a material object capable of being investigated by the methods of physical science, is thus a certain group of sense-data characterized not only by its fixity as an element in the presented whole, but also by the fact that certain of its elements (those constituting general sensibility) differ uniquely from members of other groups. The mind of the organism must then be regarded as the subject to whom the objective whole, of which the body group is a part, is presented. The relation of body and mind, at any rate for the individual concerned, is therefore simply a relation of presentation. But it was evident that more than this is generally implied in the concept of body when considered in this connection.

Before proceeding further, however, it was necessary

${ }^{1}$ The same may be said of such "commonsense" concepts as, for example, "this table," when such as the latter are considered merely as objects. But when we think of them as agents helping or hindering our purposive actions, the reference is rather beyond the sense-data to their ground. Physical science is not, of course, concerned with this subjective point of view. 
to postulate a ground of the sense-data distinct from the subject to whom they are presented. Otherwise solipsism is inevitable. Now sense-data, being objects of acquaintance, are not existents. Their mode of being, "appearance," would seem to be an ultimate one, to be distinguished from the "existence" of the subject, for example. But the ground of the sense-data must consist of existent entities which interact with the subject, and of which the sense-data are the appearance. Spiritualistic pluralism takes these entities to be other subjects at indefinitely various levels of experience; and on the basis of that hypothesis our enquiry forthwith proceeded.

As the ground of this unique group of sense-data constituting the body as a material object, we must suppose a "society" of subordinate subjects or monads, related in some peculiar way to the dominant monad with whom the body as a whole is associated. The function of these subordinates in early life is gradually to bring the dominant monad into successful touch with the environment. This they perform by means of actions learnt in past experience. In the course of the process, there is an increasing intimacy of association between mind and body, manifested in the more and more complete control gained by the former over the latter.

On the other hand, we must guard against the conception of the body as merely a communicating link standing definitely between the mind and the environment. The mind perceives and acts in and through the body, the organism interacting as a whole with the environment. Yet the body must not be regarded simply as a tool or instrument, for too great a degree 


\section{The Relation of Mind and Body}

of objectivity is thereby implied. The relation here indicated between the body and the mind cannot be adequately defined. It involves an intimacy of association between the dominant and the subordinate monads, which can best be termed the "immanence" of the former in the latter. Its nature can be realized most clearly perhaps in sense-perception and volitional movement.

The relation of the dominant monad to the environment is thus essentially a direct one, although it involves the subordinate monads. This was to be expected, for the objects of perception would certainly seem to be the appearance of the environment, and not simply of our own brain-states.

Evidently the inter-relation of the subordinate monads is constituted mainly by the immanence in them of the dominant monad. This immanence is the concrete principle in virtue of which they co-operate to secure ultimately the interests of one individual ${ }^{1}$, the dominant monad. For it is a noteworthy characteristic of organic bodies at all levels, that their reactions are marked by a reference to the organism as a whole, and not simply to some particular portion of it. Yet the fact that at very low levels it is possible for separated portions of an organism to continue to exist, together with the consideration of the physiology of the higher animals, lead ús to assume a certain decentralization of functions in the society of monads which makes up the individual organism. In other words, we must suppose the existence of sub-dominant monads and their asso-

${ }^{1}$ It does not follow, of course, that this end is consciously present in any sense to the subordinate monads. 
ciated inferiors, while in the low types of organism mentioned there is perhaps no single dominant monad, but a divided rule.

In view of the immanence of the mind in the body, it appeared likely that, while that immanence continues, there is no relation between the mind and the environment unaccompanied by any functioning on the part of the body, though in certain cases the functioning of the latter may be abnormal. On the other hand, there is no reason to believe that when the mind parts from the body at death, it is henceforth incapable of interaction with the environment.

Consideration of the points of difference and of resemblance between images and sense-impressions, together with the fact that presentations tend to be revived in the order in which they were originally attended to, led us to postulate three things. Firstly, the existence of a certain functional inertia in subjective activity; secondly, that an impression manifests by its particular quality the particular determination of the activity of the subject, by the action of other subjects, when initiated in a certain direction, while that determination is actually taking place; and thirdly, that an image manifests a particular determination of subjective activity previously established (at the time when the corresponding impression was presented), though, owing to modification by intervening activity, the determination does not keep a precisely defined form, and in time may be so modified as to be not recognizably the same. Impressions are therefore not mere subjective modifications, although dependent in part for their being on the existence of the subject, whereas

R. S. P. 
images are strictly subjective, and not the appearance of other entities. While the particular form of impressions and of the corresponding images, since it manifests the particular determination of subjective activity, is dependent in part on the body in which the subject is immanent, it yet does not follow that memory and imagination must cease at death. Granted that in this life their possibility probably depends on the possibility of the occurrence of their bodily concomitants; but when the subject is loosed from his body, he can still act in ways previously determined, and also perhaps in new ways which before were impossible.

Yet perhaps the greatest importance of the body lies in the fact that it is by means of it that we attain to the level of rational self-consciousness. Hence the body is essentially instrumental in the attainment by the individual of personality. But having served its purpose it now hampers the exercise of higher activity. The mind transcends the body more and more while yet immanent in it, but must strive without avail to render this transcendence complete, till death brings release. This growing disharmony between mind and body finds its inevitable outcome in the decay of the latter in old age; and as a consequence the powers of the mind are for the time in abeyance to a greater or less extent. But we have no reason to believe in the complete cessation of these powers after death. Rather do they regain their pristine vigour in shaking off this clay, and enter upon a new realm of possibilities. Nor does the acqui-. sition of a new body seem to be a necessity of the future life. It may be so, but let us not forget that the soul has now come to itself. Its condition cannot be com- 
pared with that of its pre-bodily existence, when the acquisition of a body was the sine qua non of any advance worth the name.

To this extent, however inadequate it be, we can indicate the nature and consequences of that unique relation which is perhaps destined ever to remain one of the greatest mysteries of life. Whether our results can in any real sense be called a solution of the problem is not easy to judge. But it may at least be claimed that it is only along some such line as we have followed, that a solution can be even approached. Yet the question is such a central one, that it introduces us at almost every step to further problems, the complete solution of which would entail a final answer to the riddle of the world. 


\section{VIII}

\section{SUBCONSCIOUSNESS AND CERTAIN ABNORMAL PHENOMENA}

\section{INTRODUCTION}

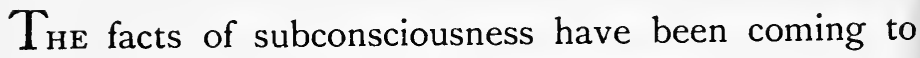
play a more and more prominent part in recent psychological investigation, and also in what is commonly called "psychical research." It is believed that processes which are in general subconscious, exercise a highly important effect on the processes which go to make up the normal consciousness of the individual. On the other hand, it is regarded as certain by those engaged in psychical research, that the phenomena which are popularly lumped together under the ambiguous title of "spiritualistic," are intimately connected with subconsciousness.

Now the psychologists have largely succeeded in formulating and co-ordinating satisfactorily the facts of subconsciousness as they are manifested in the experience of the individual. But there remains the further philosophical question as to the ultimate ontological conditions which are thus manifested. As regards psychical research, not even a satisfactory formulation of the connection between subconsciousness and spiritualistic phenomena has yet been arrived at, and the metaphysical theories propounded by many spiritualists to account for the facts, are far from exhibiting that precision of terminology and that coherence 
of statement which are necessary features of any theory capable of offering a possible solution of the problems involved. For these reasons it is of great importance to frame, if possible, a theory which shall explain the ontological facts of which subconscious and "spiritualistic" phenomena are the manifestation, and which shall provide an account of the ground of the connection between the two latter.

Before proceeding to attempt this, it must be pointed out that the term "subconscious" is a very vague one. In particular its meaning as applied to sense-impressions is quite different from its meaning as applied to images. There is a region of the sensory continuum of which we are not distinctly conscious, for various reasons, although we cannot be said to be unconscious of it-in fact, it certainly modifies by its presence that part of the continuum of which we are distinctly conscious. The impressions which make up this "subconscious" region can best be described as being "beyond the threshold" of consciousness, that is, they are ultraliminal'. The same cannot be said, however, of the ideational continuum. Images to which we are not at the moment attending, although we are capable of reviving them, do not form a region of the continuum beyond that of which we are at the time distinctly conscious. They are rather, as it were, implicitly involved in the consciousness of the individual though not explicitly presented. But they are yet capable of modifying what is presented, e.g., as when a train of thought is carried on without the distinct presentation of the corresponding images. Such images are below the threshold of con-

${ }^{1}$ Cf. James Ward, Psychological Principles, pp. $9 \circ \mathrm{ff}$. 
sciousness-they are subliminal ${ }^{1}$. We have, then, to distinguish carefully in subconsciousness between ultraliminal sense-impressions and subliminal images.

It appears necessary, therefore, to provide first of all an hypothesis which shall be capable of giving a comprehensible account of the real nature of what are manifested in experience as ultraliminal impressions and subliminal images respectively. In each case, a brief description of the accepted psychological facts may serve as a preface to the discussion of the ultimate ground of those facts. Before we can find in that ground a basis of interpretation of the abnormal phenomena with which our further enquiry will be concerned, an investigation must be made of what may perhaps be termed the connecting link between these phenomena and those characteristic of the ordinary normal life of the individual. This connecting link is frequently referred to by a term which has latterly become very widely used-and almost as widely abused-namely the "subliminal self." This term is used in several different meanings, only one of which is strictly correct. It is important to determine the latter, and also to discover what, if anything, may really be indicated by the term when used in other connections. Our enquiry may then conclude with an examination of the various types of abnormal phenomena investigated in psychical research, and an attempt to unify and co-ordinate them by means of an hypothesis based on the results obtained in the preceding discussion.

Before closing these introductory remarks, it may perhaps be well to anticipate a possible objection based ${ }^{1}$ James Ward, Psychological Principles, pp. 94 ff. 
on the ground of lack of evidence for the phenomena above alluded to. No doubt trickery has been widely practised. But one can only say that the body of evidence now produced and attested by men trained to scientific methods of experimentation and criticism of the highest order of precision, is so overwhelming, that anyone who pretends to an open mind cannot help but accept what has been so carefully determined and laboriously recorded, as being in general of the same order of certainty as other more ordinary phenomena investigated by science, whatever may be true of any particular case. The facts thus accepted are of a nature bearing so closely on the most pressing of human interests that, as previously stated, it is of the highest importance to reach if possible some coherent and comprehensive account of their ultimate ground in the structure of the Universe.

\section{Ultraliminal Impressions}

Within the region of which we are normally conscious at any instant, there is but one comparatively restricted part which can really be said to be distinctly presented to us, namely the part to which we happen to be attending at the given instant. This small distinct region within the field of consciousness has been termed the "focus" of consciousness. Surrounding it (to use a spatial metaphor) is a very much larger region which cannot be said to be distinctly presented to us, although we are quite conscious of it. Yet the focus of consciousness can be voluntarily transferred to any part of this larger region. Accordingly it is to the latter 
that the name "field of consciousness" may be legitimately given. This, however, does not exhaust the presented whole. Beyond the field of consciousness there is a further region to be considered. It is distinguished by the fact that no part of it is capable at the time considered of becoming the focus of consciousness. Its intensity is not sufficient to disturb appreciably the existing distribution of attention. Yet we cannot be said to be unconscious of it. It modifies the presented whole rather in the manner of a vague background, and it may perhaps best be termed provisionally "the field of subconsciousness." It is separated from the field of consciousness by a comparatively narrow region (though not indefinitely narrow) which is termed the "threshold." Accordingly, sense-impressions forming part of the field of subconsciousness are called "ultraliminal."

Briefly, then, the psychological facts here concerned are as follows: The presented whole consists of a small, distinct region (the focus of consciousness) beyond which is a much larger region (the field of consciousness) any part of which may become the focus of consciousness. Beyond this again, and separated from it by a narrow, rather ill-defined belt (the "threshold"), there is a further region of indefinite extent (the field of subconsciousness). The distinctive difference between the fields of consciousness and of subconsciousness respectively at any instant is that while any part of the former is capable at that instant of becoming the focus of consciousness, parts of the latter are not. But it should be noted, finally, that regions of the presented whole which at one time form portions of the field of 
subconsciousness, may at another time form portions of the field of consciousness, and vice versa. That is, any impression, whether ultra- or infra-liminal, may traverse the threshold of consciousness.

We must now attempt to obtain a satisfactory hypothesis of the real nature of that process which consists, in experience, in the cognizing of a senseimpression. If we adopt a spiritual pluralism as our ontological system (and this will be done here), the object of an individual experience is the manifestation to the subject of that experience of the existence of other subjects, and the changes in the object are the manifestation to the subject of his interaction with other subjects and its results. Now even though it be supposed that subjective activity is essentially of one type (namely what, in experience, we call "attention"), yet the form taken by the activity in any given instance will depend partly on the nature of the subject himself, but partly also on the nature of the subjects with whom he is interacting. In sense-experience, then, the presented object is the manifestation of the form which the subject's activity is taking, while that form is actually in process of determination by his interaction with other subjects. We may further suppose that, when the subject's activity is, at some future time, again directed the same way, as it were (to use a crude spatial metaphor for want of a better), it will maintain more or less the same form to which it was previously determined when directed that way, although the other subjects, interaction with whom resulted in the given determination, are no longer present in the same sense. In this case the manifestation of the determinate 
activity takes the form of an image as opposed to an impression?

For each subject, then, the object of sense-experience is the manifestation of his interaction with other subjects. Now a mere plurality of individual subjects cannot contain within itself the ground of this interaction whereby the many subjects constitute a universe. As an ontological hypothesis, pluralism, though it may carry us far, is incomplete. It requires to be supplemented by postulating the existence of a single universal entity, in which the many exist, and which provides the ground of their interaction. We are not here concerned with the nature of this entity, nor with the rigorous demonstration of its necessity. Suffice it to say that we have in this case a question of all or none. There must be a single such entity, or the difficulty of mere plurality remains. In the absence of this universal ground none of the subjects or monads could interact. But if it is present all must interact-each continuously with every other.

We have here, in fact, an idea which was foreshadowed by Leibniz when he spoke of each monad as mirroring all the rest of the universe from a particular point of view. From our hypothesis (which can only be tested by its power to deal with particular problems such as those, for example, we are here considering) it follows that at all times each monad acts and is acted upon by every other, to a greater or less extent. It is in this fact that the ground of the field of subconscious sense-impressions consists. The latter is the manifes-

${ }^{1}$ This is dealt with more fully in the essay on "The Relation of Mind and Body," Section V. 
tation or appearance to the subject concerned of the vast majority of other subjects which go to make up the universe. Its relations with them at the time considered are not such as to constitute the conditions (whatever they may be) necessary for interaction "intense" enough (if the word may here be used analogically) to be manifested by the occurrence of a sense-impression above the threshold of consciousness. With only a comparative minority of other individuals will the interaction of the given individual be of the order requisite to be manifested as a normal senseimpression above the threshold. It is the appearance of this minority of other subjects which constitutes the field of consciousness (as distinct from sub-consciousness) of the given subject.

Without entering in any detail into the ontological conditions necessary for the occurrence of a senseimpression above the threshold, it may yet be remarked that it follows from the fact that the latter has some "breadth," that the conditions required have not a hard and fast limit. There will in fact be a noumenal continuity corresponding to the phenomenal continuity. Moreover, it is evident that spatial and temporal relations and characteristics must constitute the manifestation in experience of an important (perhaps the most important) part of the noumenal conditions involved. From our theory, when an individual passes "out of sight," as we say, owing to phenomenal spatial conditions, it follows that he is still manifested to us in some way (though we do not distinctly apprehend the fact) in the field of subconsciousness, whereas previously the impression which manifested him was in the field of consciousness. 


\section{Subconsciousness and Abnormal Phenomena}

In general, the only conditions which will bring about a re-lifting of the impression above the threshold, are those manifested in a reversal of the spatial conditions referred to. But there is no reason why in certain cases other fresh conditions should not intervene which are by themselves capable of producing a manifestation of the individual above the threshold, though not necessarily in the same form as before, without the presence of those conditions manifested in the reversal of all the spatial conditions.

The foregoing are the essential points of the theory to be used later in dealing with abnormal phenomena. But before approaching the latter, we must attempt to make the theory more complete by a consideration of the nature and ground of subliminal images. To that we may now proceed.

\section{Subliminal Images}

Of the multitudinous variety of images any one of which an individual subject is capable of reviving at any time in general, that he wishes, by far the greater portion are not, at a given instant, presented with any degree of distinctness. Yet in view of the capability of revival, it seems highly unlikely that in the interval between instants at which it is attended to so that it can be distinctly apprehended, an image ceases altogether to form part of the presentational whole. Rather must it be supposed, as we have seen, that the image is for the time being below the threshold of consciousness, that is subliminal. If this be so, however, we should expect that it would yet make its presence felt in some 
degree. That the latter is the case can be realized by considering how (to make the point more obvious by an extreme example) a past bitterly painful experience modifies for a time the whole succeeding experiences, even when the images which recall it are in no way present above the threshold. Moreover, we realize the presence of such subliminal images when we attempt to recall one distinctly, and for the moment it escapes us. We know that it is there, but it will not rise above the threshold in spite of our efforts, until suddenly, perhaps when we have given it up and are attending to something else, it rises distinctly into the field of consciousness.

As opposed to their orderly sequence as they unfold above the threshold, images are sometimes said, while subliminal, to be "involved" or "implicit" in the experience of the individual concerned. Nor do they, while in this condition, necessarily remain quite disconnected, but frequently gather to form a complex which, under suitable conditions, rises towards the threshold as a whole, constituting what Wundt has termed an "apperception-mass." Yet why should images and complexes of images remain below the threshold at all? Why should they not remain as definite parts of the presented whole, within the field of consciousness ?--To this it is replied that their rising above the threshold is inhibited by other psychical processes and functions, but the explanation does not carry us far so long as we confine our attention to the object of experience alone. To advance further we must frame an ontological account of imagination which shall explain the facts described in this brief outline of the psychology of subliminal images, and in particular 


\section{Subconsciousness and Abnormal Phenomena}

the fact of inhibition which secures the retention of the majority of images below the threshold of consciousness.

In considering sense-impressions, we adopted the hypothesis that they are manifestations to the subject of the determination of his activity to particular forms by interaction with other subjects, while that interaction (and therefore the determination) is actually taking place. Once determined to any particular form, the activity will in future, when directed the same way ${ }^{1}$, as it were, again, take on the same form, even though the other agents who played a part in its original determination are no longer interacting with the given subject as they were. The result is manifested to the subject by an image ${ }^{2}$ which resembles the original impression.

Now the image which thus manifests to the subject the form of his activity will evidently be supraliminal, for he is acting at the moment in the particular way considered. But in the intervals when he is not acting thus, there is still in his nature a tendency for his activity to take that particular form when directed appropriately. It is the continued existence of this tendency, which is not at the time issuing in actuality, that may be supposed to be manifested by a subliminal image. The rising of such an image above the threshold, is, then, the manifestation of the passing over of a tendency to activity in a particular form, into actuality.

${ }^{1}$ It is not easy to find a satisfactory terminology in this connection, without recourse to spatial metaphor. The direction of activity may be voluntarily determined although its consequent form depends on interaction. In experience we frequently direct our attention a certain "way," without knowing, for example, what we shall perceive.

${ }^{2}$ Cf. "The Relation of Mind and Body," Section V. 
It is important to remember, at this point, that in spite of the manifold diversities of experience, the subject himself remains one and indivisible. It accordingly follows that the various actions and tendencies to action we have been considering, must continuously modify one another. In particular, during the interval between the presentation of an impression and the subsequent revival of the image, the tendency to a particular form of activity which the latter manifested while still subliminal will have been modified by intervening interactions. Hence, when at last the tendency issues in actuality, and the image rises above the threshold, the form of the activity (manifested by the nature of the image) will differ to a greater or less extent from that to which it was originally determined. This is manifested by differences between the image and the original impression. In addition to particular differences there is the notable universal difference consisting in the fact that images are always more general and vaguely defined than impressions. This may be regarded as manifesting the modification of the original form of the activity by other acts and tendencies ${ }^{1}$. The activity, originally specialized in form and "concentrated," as it were, by the interaction with others, afterwards becomes less specialized and more "diffused" on account of the modifying influences referred to. This modification may be carried to such a point that the image can no longer be

${ }^{1}$ It is in this that the ground of the "apperception-masses" previously referred to consists. An image-complex manifests a form of activity which is the result of the mutual modification of various actions and tendencies. 
revived in a form recognizable as the original-and we forget.

The reason for the inhibition of subliminal images should now be clear. A subliminal image manifests a tendency to a particular form of activity. But while other forms of activity hold sway, this tendency cannot issue in actuality. Only when the former are suspended will the strongest tendency pass into actuality, and the image which manifests it rise above the threshold. Yet a tendency may be so powerful in some cases as to become actual, overcoming other forms of activity although the latter may be in process of occurrence at the time.

The nature of the subject, as modified by his interaction with other subjects, consists, then, in the tendencies which his activity has to take particular forms, and it is in the resultant acts, whose forms are manifested by supraliminal images, that his nature is explicitly revealed to himself, so to speak, and, when the images lead to movements, his nature is revealed to others. But of the subliminal images which manifest his tendencies, there is one group of particular importance. This we shall now consider.

\section{The (so-called) Subliminal Self}

The idea of what is commonly called the "subliminal self" has rapidly developed of recent years, for it is regarded as a conception whereby the reasons for certain types of abnormal behaviour on the part of the individual can be co-ordinated and unified. These peculiar types of behaviour fall broadly into two classes. In the first place, it is observed that under certain 
appropriate conditions bodily actions are performed of which the subject is apparently unconscious. Nor are these movements merely such as occur in ordinary reflex action. They are frequently very complex, as, for example, when they consist in spoken sounds or purposive movements of the limbs indicating the carrying on of a definite line of thought or action similar to those normally carried on by the subject of which he is fully conscious. In some cases, on the other hand, the subject is conscious of the unusual actions at the time, although unable to exercise normal control over them. When the abnormal state has passed, the events which took place while it supervened are not, in general, consciously remembered by the subject, though this evanescence does not appear to be the invariable rule.

The second type of abnormal behaviour referred to, consists in the capability possessed by some individuals (and perhaps by all to some extent) of perceiving in certain circumstances objects which would not normally be perceived in those circumstances owing to prohibitive conditions of (for example) space and time. During the occurrence of this abnormal perception the subject is fully conscious and capable in every way; in fact, the difference from normal perception lies not in what is perceived nor (directly) in the manner of perceiving it, but in other accompanying conditions.

To account for phenomena of this kind, the concept of a "subliminal self" is employed. It is not easy to determine the precise significance attached to this term by those who use it most freely. Perhaps the essence of the idea involved may be put roughly somewhat as

$$
\text { R. S. P. }
$$


follows: There exists in the case of each individual a "part" of the self which normally has no direct share in the control and direction of his activity. But in certain circumstances, that dominant "part" of the self which is in control during normal consciousness, is in temporary abeyance, while the other, normally "subliminal," part takes control. The nature and extent of the latter is indefinite, but even during normal consciousness the influence of the subliminal self is felt to a greater or less extent. Moreover, the subliminal self may possess "faculties" which the normal self is not capable of exercising, and it is these, perhaps, which hold the field in cases of abnormal perception.

Such a view as that just stated evidently involves serious assumptions, and therefore calls for careful criticism. And, first of all, it is necessary to distinguish between the term "subject" which has only one legitimate meaning, and the term "self" which has at least two. "Self" may, for instance, refer to the subject of the experience considered, or it may refer to the empirical ego or "me," which forms a part of the object of that experience. Much turns on this distinction, as we shall shortly see.

It is first necessary to emphasize the fact that the subject of experience is an indivisible unity. In no valid sense can he be said to be a whole of parts. Nor can it be objected that we have been speaking of many diverse activities and tendencies of the subject, and that this implies that he has parts. For although the conditions of discursive exposition make it necessary to describe the state of the subject in this way, it must not be forgotten that actually this state is one indivisible thing, 
namely what may be described by analogy as the "resultant" of the various activities and tendencies into which we are compelled to analyze it for the purpose of discursive expression. We have the counterpart on the objective side of this unity of the subject, in the unity of the presentational whole ${ }^{1}$. In particular, when we come to analyze the latter we find that to only one restricted region can the term "focus of attention" be applied. Thus one subject implies in the presented object one, and only one, focus of attention and vice versa. This is a most important point.

It is clear, then, that the term "subliminal self" cannot be held to refer to a part of the subject of experience, for the latter is not a whole of parts. Hence, if the term is to have a valid significance, the word "self" must here be regarded as applying to that complex in the object of experience which makes up the empirical ego or "me." For even though the categories of whole and part may not ultimately be adequate to express the unity of the object of experience, we may at any rate be able to discover here a valid interpretation of the fact that our analysis, even though it yields an approximation only, compels us to distinguish, if we are to reflect at all, just such-and-such parts and no others. In this case the particular part of the object which calls for consideration is the complex forming the empirical ego. But analysis (even an approximate one) discovers nothing of this kind in the subject of experience. We may speak there of actions and tendencies, but throughout they are necessarily con-

${ }^{1}$ See "Scientific Method in Philosophy and the Foundations of Pluralism," Section VI, and "Immortality," Section IV. 
sidered as actions and tendencies of a single individual unity.

Returning to the empirical ego, there is every reason to suppose that, while of the images forming part of it many are normally supraliminal, a considerable (and perhaps very great) proportion remain subliminal in all ordinary circumstances. Now the images forming the empirical ego are distinguished from other images by the fact that, for the most part, they represent the individual as acting in various ways. Hence the determinate forms of activity which they manifest are important by reason of the fact that they generally lead to those other forms of activity which are manifested by movements. Thus, in its ontological aspect, the subliminal self is a certain group of subjective tendencies which do not normally issue in activity, although when they do become actual, the other forms of activity to which they lead are manifested by movements unusual either in their own nature or by reason of accompanying circumstances. This appears to be the only strictly valid meaning of the term "subliminal self." The latter is not a "part" of the subject of which the normal self is another "part." It is simply a particular group of tendencies to activity of the one indivisible subject, who normally acts in other ways. Otherwise, we should be committed to regarding any given subject as made up of two or more other subjects.

The case would seem to be different, however, when the subject is unconscious of certain of his bodily movements. The latter may then perhaps be grounded in the activity of other subjects, whether forming part of the organism of the given subject or not. In this con- 
nection cases of dual trains of activity call for special consideration. The problems here raised will be investigated for particular types of abnormal phenomena later. For the moment it will suffice to say that where movements are due to the activity of a subject other than the dominant monad of the organism, this other subject cannot be described as the subliminal self of the dominant monad, for it is a subject distinct from the latter and will have its own subliminal self. The case is entirely different from that considered above, and the distinction must be kept clear. Moreover, there is a third case in instances of abnormal perception, but here it is evident that, whatever the ground of the presented object, the percipient subject concerned is the dominant monad of the organism. Neither the subliminal self nor monads other than the given dominant are here concerned on the subjective side. Keeping such considerations in mind, we may now go on to the investigation of particular cases.

\section{Abnormal Phenomena}

In dealing with cases of abnormal phenomena, it will perhaps be best to commence with those which depart least from ordinary types and gradually proceed to others exhibiting more and more unusual characteristics. For even in extreme cases it generally (but not always) appears that the observed phenomena can be related to phenomena which are more or less normal, by exhibiting a continuous process whereby the former develop gradually from the latter. We may therefore select as a starting point a type of phenomena of so 


\section{Subconsciousness and Abnormal Phenomena}

universal and common occurrence, that it may hardly be described as abnormal at all, namely dreaming.

(a) Dreams. Sleep consists in the (voluntary or involuntary) withdrawal of attention from objects normally presented, whether the latter be sense-impressions (including organic sensations) or images. That means, as we have seen, that normal forms of activity are temporarily suspended. But in the dream-experiences of the individual the subject concerned is the same as the subject of waking consciousness. The objects presented, however, differ in general very considerably from those normally presented, both in degree of correlation and consistency, and in their actual nature. During sleep memories wholly or half forgotten when waking, revive, sometimes with remarkable distinctness. Images recur of sense-impressions which, when perceived in waking hours, we had perhaps scarcely noticed. And again, we dream that we are acting with an end in view strongly desired, but as strongly suppressed for some reason or other when awake.

On the whole, we may suppose these dream-objects to be classed as images. They appear, perhaps, more distinct than ordinary images because we are not at the same time conscious of sense-impressions wherewith we compare them. Nor is the reason for their peculiar nature far to seek. With the cessation of normal forms of activity, a chance is given to those latent tendencies, which are manifested, according to our hypothesis, by subliminal images, to become actual. This result is manifested by the rising of those images above the threshold, and we dream. Memory-images "crowded out" normally by more intense presentations, desires 
thwarted previously by being held in firm check, now, in the absence of the inhibiting forms of activity, hold the field.

We saw that the tendencies which are manifested by subliminal images, are, by reason of the conditions to which they are due and the modifying influence of other forms of activity, comparatively loosely co-ordinated. Consequently the groups of images which manifest them lack the coherence of those other groups of images to which we attend when following accustomed trains of thought or imagination. This is the reason why dreams, in general, are noted for their peculiar incoherence, and quick transitions from one group of images to another almost entirely disconnected with it.

In dreams, that particular group of tendencies which, as we have seen, constitutes the subliminal self, plays a very prominent part. The subject of the dream-experience is, we pointed out, identical with the subject of waking experience. But we frequently dream of ourselves as acting in certain rôles very different from those to which we are compelled by circumstance in waking life. Especially is this the case when we have wished or imagined ourselves to be heroes or victims of such adventures. Moreover, analysis of dreams renders it highly probable that all the personages taking part therein are really objectifications of the subliminal self of the individual who is dreaming.

There remains the question as to whether all dreampresentations are of the nature of images or whether some should not rather be classed as sense-impressions. Certainly when a door bangs and we dream of an explosion, one of the presentations concerned is a sense- 
impression. This, not being associated with the other impressions normally accompanying the banging of a door, is followed instead by the rising of an imagecomplex associated with that particular kind of impression. The case of the occurrence in dreams of impressions of this type has, however, no bearing of any great importance on general questions. But there is another possibility of real importance, namely as to whether, during sleep, it is possible for other subjects to act upon us. When, for example, we dream vividly of any particular person, may it not be that an interaction is really taking place between him and us? In this case, the presentations involved would be classed as senseimpressions. Certainly they are not accompanied (so far as we can tell) by the usual peripheral changes, though there may perhaps be cerebral accompaniments. But, in any case, the presentations would be manifestations of the determination of our activity by interaction with one or more other subjects, and not of the occurrence of a form of our activity previously determined. Hence they would display the general characteristics of senseimpressions. However, the possibility of the occurrence of such interaction evidently depends on the wider problem of abnormal perception, and we shall therefore consider it in the section given to the discussion of that problem.

In dreams, then, we find manifested the occurrence of forms of activity which in waking life are present simply as tendencies. Their actualization is due to the cessation of the usual forms of activity. In sleep the conditions of this cessation are normal. We have now to consider a similar cessation of activity, but 
in this case the conditions producing it are distinctly abnormal.

(b) Hypnotism. The hypnotic trance, while exhibiting certain important features similar to those of ordinary sleep, is nevertheless characterized by others no less important which differ from anything observed in the case of the latter. A scrutiny of the various methods used to induce the trance-condition, seems to show that all consist essentially in bringing about the withdrawal of attention from normal objects, either by direct instructions to assume a "vacant" state of mind as far as possible, or by causing the subject to fix his attention continuously on one object alone, until a condition of fatigue supervenes in which most normal objects pass from the field of consciousness. The necessary withdrawal of attention is greatly aided if there is expectation of trance ; and, indeed, if there is little or no comprehension of what is expected, it may be quite impossible to induce the condition. Young children and idiots are particularly difficult to hypnotize.

Trance is not always required to bring about the desired results. Repeated suggestion, especially in the case of the subjects of previous experiments, will sometimes be sufficient alone. But evidently the same result is here produced, namely the withdrawal of attention from certain normal presentations and its redirection to objects which may be decidedly abnormal. Nor can we be quite certain that even in such circumstances there is not a rapidly evanescent state of trance. In any case, however, there is no doubt that the hypnotic trance is a condition intermediate between waking and deep sleep. The operator engages the subject's attention sufficiently 
to keep him from the latter, but not so much as to wake him completely. This is borne out by the fact that a hypnotized subject, if left to himself, either wakes right up, or falls into a profound slumber.

Edmund Gurney distinguished three stages in the hypnotic trance. There is at first what is called an alert stage. The subject remains conscious of practically all that is happening around him, and is sensitive to pain. This condition frequently passes into the second, or deep stage. The subject is now insensitive to pain, and appears not to notice what is happening around him, although he remains mentally active. Finally, profound sleep supervenes. In connection with these different stages, it has been observed that after the lighter ones the subject retains the memory of most that has happened during the trance. This is not the case, however, with the deeper stages. After recovery from these, the memory-images associated with them remain subliminal, and cannot be raised above the threshold by any ordinary means. In the trance, however, the subject remembers what has previously happened during that trance, and displays, in fact, a considerable amount of spontaneity; but this always consists in the development of suggestions made to him, and not in that of ideas contrary to those suggestions, which would certainly inhibit the results of the latter were the subject normally conscious.

On the hypothesis we are adopting, the general explanation of the hypnotic state will be somewhat as follows: The conditions which precede the onset of the trance are such as to result in the cessation of normal forms of activity to a more or less considerable extent. Accordingly, tendencies which are ordinarily 
latent are now capable of actualizing without difficulty. This is manifested by the readiness with which images rise above the threshold, that normally remain subliminal. Moreover, the form of the resulting activity will follow any pre-determined course ${ }^{1}$, that is, the images which manifest it will be succeeded by others previously associated with them, although such association would perhaps have been inhibited in the normal state of consciousness. This latter is frequently the case when the association is between images and movements.

The hypnotic condition differs from sleep in that certain normal forms of activity still continue. This is manifested by the fact that certain sense-impressions are still perceived. On the other hand, these senseimpressions are not in general followed by the images and movements normally associated with them. In other words, although the form of the subject's activity is still being determined by interaction with others in the usual way, the form so determined does not develop in the manner in which it has been previously accustomed to develop. It will take any direction at the suggestion of the operator. This fact accounts for the peculiarity of the actions which are frequently performed. In the deeper stages, perception diminishes to a continually increasing extent, and the only method by which the operator can lead the subject to act in a desired way, is by ideas directly suggested to him and not by statements about surrounding objects. With this gradual suspension of all normal forms of activity, the trance passes into sleep.

${ }^{1}$ See the essay on "The Relation of Mind and Body," Section V, for a detailed discussion of this point. 
The strange, and often ludicrous, actions performed by hypnotized subjects are explained by the temporary absence from the field of consciousness of the rational image- and thought-complexes which normally guide and restrain subjective activity. The images now supraliminal are those which in ordinary consciousness remain subliminal. They manifest diffuse and loosely co-ordinated tendencies, which readily develop in any direction. The factors which would usually inhibit development in certain directions are, for the time being, in abeyance. The forms of activity manifested in the hypnotic trance are, in fact, specially characterized by the fact that they are very often the outcome of tendencies habitually repressed in waking life.

The foregoing is an explanation in outline of the facts of hypnotism. But to clear the matter up as completely as possible, it will be more satisfactory to consider in somewhat greater detail the more outstanding phenomena associated with the hypnotic state.

In the first place, the extreme suggestibility manifested by the hypnotized subject is due to the fact that if a train of activity be initiated, it will continue, owing to functional inertia ${ }^{1}$, on any course which it has previously taken, for other forms of activity which might normally inhibit it are suspended. Thus any senseimpression perceived by the subject through the action or speech of the operator, will be succeeded by a sequence of associated presentations, even though such associations are ordinarily suppressed and remain below the threshold. Hence the idea of a movement, if suggested to the subject, will be immediately followed on his part

${ }^{1}$ Cf. passage referred to in the last note. 
by that movement, even though the latter be painful or ludicrous. Thus suggestibility consists simply in the readiness with which trains of activity are carried on in the absence of the normal inhibitory factors. In waking life we imagine many movements, desirable and undesirable, which we do not actually execute. But in the trance, a suggestion which results in the presentation of a movement, is inevitably followed by that movement.

An important class of phenomena occurring in this connection, is that commonly known as "cure by suggestion." Such cures may be roughly divided into those concerned with mental, and those concerned with physical ailments. There is little difficulty in accounting for the former. Mental diseases consist essentially in the setting up of strong tendencies in subjective activity to take certain harmful forms. By inducing trance, these forms of activity are suspended, and resume temporarily the status of latent tendencies. It is then possible to set up other forms of activity which, owing to the peculiar condition of the subject, may be fixed so firmly that on the return to normal consciousness, they remain as tendencies powerful enough successfully to oppose and even to prevent entirely the actualization of the original harmful tendencies.

Physical cures appear, however, to strike deeper. Evidently they depend on the relation between the subject and his body. Physical disease is the manifestation of a disturbance in the relations between the monads composing the latter. In view of the immanence of the subject or dominant monad ${ }^{1}$, this occurrence is manifested

${ }^{1}$ See the previous essay, Section IV. 


\section{Subconsciousness and Abnormal Phenomena}

to him by the presentation of painful organic sensations. But it is also an evident consequence of his immanence, that the activity of the subject must exercise considerable influence on the adjustment of relations between the inferior monads. Now the form of subjective activity which will lead to a harmonious readjustment of the disturbed relations, must be manifested by the occurrence in the subject's experience of certain presentations occupying the focus of attention. The setting up of this activity will be hindered by the action of the disturbed monads themselves. This appears in the experience of the dominant monad as the insistency with which the painful presentations occupy the focus of attention, thus resulting in a form of subjective activity which is positively harmful. The inducing of trance, however, will ensure the cessation of the activity in this form, and it may then be possible to redirect it in a form which will bring about successfully the requisite readjustment of conditions among the inferior monads. Thus every organism, considered as a whole, contains to a greater or less extent the ground of its own recovery from diseases even the most serious. Nevertheless, it may be regarded as doubtful whether extreme cases, such, for example, as the miracles of Lourdes, are completely explicable in this way. Judgment on the question depends on exact knowledge of the patient's mental condition at the time. There might perhaps exist in every case a condition of the nature of trance.

Of somewhat similar nature to the foregoing are the insensibility to pain and the immunity from harm consequent upon serious burns or abrasions, frequently reported in hypnotic cases. The insensibility and the 
immunity are closely connected, for it has been shown that pain itself acts as an irritant to the very causes producing it. Hence if the attention be withdrawn from the painful sensation, serious consequences of the hurt may be avoided. Evidently this depends on the reaction of the organism as a whole $e^{1}$ to the cause of the trouble. Provided the activity of the dominant monad is not improperly directed, the inferior monads in whom he is immanent, will be able to bring about the necessary readjustment. This readjustment may be actively helped by the dominant monad, if he turns his attention to appropriate objects, that is, if he directs his activity in forms opposed to those manifested by the painful sensation. On the other hand, where there has been no harm of any kind he may yet, by directing his activity so that it takes certain forms (manifested by the presentation of images of painful impressions), so disturb the relations of the inferior monads composing his body, that scars or other hurts appear in spite of the absence of external causes. A well-known example of this is provided by the stigmata reported to have appeared on the bodies of certain saints in the past.

We now come to a somewhat different kind of occurrence. By appropriate suggestion, it is possible to cause vivid hallucinations to appear to the hypnotized subject both during the trance, and also at some future time after the latter has ceased. Hallucination during trance is not difficult to explain. By suitable action on the part of the operator, the activity of the subject may

1 The organism as a whole consists of the society of inferior monads in whom the dominant monad or subject is immanent. See previous essay, Section IV. 
be led to take any particular form, in the absence of other inhibiting forms, and the image which manifests this to the subject will be correspondingly vivid in the general absence of competing images and impressions. In a similar way, hallucination after trance may be produced by setting up a strong tendency on the part of the subject's activity to take a certain form. Owing to the lack of disturbing factors during trance, such tendencies may be made very strong indeed. At the required time after the trance, the image manifesting the given form of activity will rise above the threshold as the tendency passes into actuality, and owing to the strength of the tendency, a vivid hallucination will be the result. In an exactly similar way, not only may the subject be made to perceive certain images at a given time after the trance, but also to perform certain movements.

But why should the image or the movement become supraliminal at exactly the right time? If strong tendencies are set up during the trance, why should they not actualize at any time subsequent to the latter? The reason is clear when we reflect that (in spite of the metaphorical way of expressing matters to which we are frequently compelled by the exigencies of discursive thought) these tendencies, being subjective factors, do not possess temporal characteristics at all. The latter are possessed only by elements in the object of experience ${ }^{1}$. Hence we have a non-temporal tendency manifested by a subliminal image which has, as one essential feature, a certain fixed temporal character, such

${ }^{1}$ Cf. "Immortality," Sections II and III, and "Scientific Method in Philosophy, etc.," Section VIII. 
that it rises above the threshold with the required temporal sign and standing in the required temporal relations. Regarded in its true light, by viewing the object of experience as a whole, we have, not an image or movement at a particular time, but one element of the objective whole, namely an image with certain temporal features.

Evidently the point we have just been discussing is closely connected with what is called "the appreciation of time" manifested by the subject during trance, and subconsciously in the period following it. Probably the general explanation of the extraordinarily exact appreciation recorded, is that in ordinary waking life, the incidents which mark the flux of time are continuously registered in subconsciousness by ultraliminal impressions, with a kind of rhythm. The activity thus manifested culminates in a peculiarly strong subjective tendency. During trance, this tendency is stimulated by suggestion, peculiar emphasis, so far as the phenomenal side of the matter goes, being laid on the end of the period of waiting, namely the moment at which the hallucination is to appear, or the movement to be performed. But in particular cases, there is no doubt that this account needs supplementing by special explanatory reasons. For example, the subject sometimes perceives the face of a clock, which registers the time fixed upon ${ }^{1}$. In some cases this is probably an associated image, but in others it seems rather to point to abnormal perception of an actual clockface, described by the subject, which may perhaps be out of sight and at a considerable distance. Very probably this is due to an ultraliminal

${ }^{1}$ Cf. Psychical Research, by Sir W. F. Barrett, p. 93.

R. S. P. 
impression, which would normally remain beyond the threshold, becoming infraliminal, the appropriate impression being determined by association with the matter in hand. As a matter of fact, abnormal perception has frequently been observed during trance. This is probably accounted for by the readiness with which the subject's attention may be caused by suggestion to withdraw from those impressions which would normally be infraliminal. It may then be redirected to impressions which would normally be ultraliminal. But we may leave the fuller consideration of facts of this kind till the discussion of abnormal perception in general.

Another peculiar feature of the hypnotic trance, is the remarkable increase often noticed in certain intellectual activities. An examination of the various cases recorded, however, shows that this quickening of activity only takes place in certain directions, and these are not on a higher level (in fact they are generally on a lower) than those manifested by the individual in his normal state. In the case of that dual activity, which seems to be the commonest example of what we are now considering, one train of activity involved appears always to be merely habitual. For example, the subject will sometimes perform a mathematical operation and at the same time count aloud up to roo. But it is an everyday fact of ordinary life that we can do two things at once, without both series of impressions or images involved occupying the focus of consciousness. In such cases, one set of actions will of course be habitual. Now in the trance, it is possible to confine activity to comparatively few forms. Hence this power of doing two 
things at once will be greatly enhanced, and may extend to trains of activity which could not be carried on together in waking life, for although one may be largely habitual it may also be very complicated, and the distractions of waking life are many. On the other hand, there is no doubt that during trance dual trains of activity sometimes occur, both of which imply original and not merely habitual action. The subject will sometimes carry on a conversation, while his hand writes "automatically" of different matters. Here two distinct trains of thought are involved, implying two foci of consciousness, and therefore two subjects ${ }^{1}$. We have, in fact, a special case of automatic writing, and the latter will call for separate consideration later.

Before leaving the discussion of hypnotism, we may refer to the idea conceived by Gurney, of "tapping" the subject's subliminal state, after a trance, by means of automatic writing, a planchette or similar instrument being used. Generally, the writing refers to orders given during the trance, and is thus simply a means of allowing tendencies set up during trance, or other tendencies closely associated with them, to actualize without clashing with normal forms of activity. In this case, only one focus of consciousness, and therefore only one subject, is necessarily involved. When, however, the writing refers to original matters, we come back to the case mentioned in the last paragraph.

Leaving for the moment this question of automatic writing with which our investigation of hypnotism closes, we shall next go on to consider a type of phenomena which may perhaps prove in some cases to constitute

${ }^{1}$ Cf. above, p. 259. 
a link between the two, namely what is referred to as "multiple personality."

(c) Multiple Personality. Cases of this kind have been reported to exist from time immemorial. It was formerly the fashion to regard them as exclusively due to the temporary possession of the body by demons or evil spirits. Whether there be any truth in this version or not, we are at least certain nowadays that many of these cases can be satisfactorily explained without invoking the aid of such powers.

The facts of multiple personality are briefly as follows: It sometimes happens that an individual commences, more or less suddenly, to act in a way totally unlike anything that might be expected from his previous actions. Nor are these acts isolated. The whole routine of his life is changed. In character, tastes, emotions, in fact in all that goes to make up personality, the man appears changed. $\mathrm{He}$ is described as being a different person. In course of time, unless insanity or some other morbid condition supervenes, there may be a sudden or a gradual transition back to the original state, and the man "becomes himself" again. Memory of the one state may or may not be retained during the other state. Generally it is not.

There are certain important psychological facts worthy of mention as bearing directly or indirectly on cases of multiple personality. It is well known that radical change in the body-sensations due to any cause, may not only alter fundamentally the concept of body entertained by the subject concerned, but also the concept of self. Especially is the latter the case, when the changes in bodily sensations are such as to affect 
considerably the general emotional tone. The individual, as he himself frequently describes it, "feels a different man"; and this difference will often far outstrip, in degree and in kind, such normal differences as that between sickness and health.

Another very important fact is that, even in the most sudden changes of personality, the change seems always to be preceded by a transition period of unconsciousness, however short. Frequently, indeed, the change is first apparent on awaking after a night's sleep. A further fact somewhat closely connected with this, is that the various personalities may be evoked by suitable suggestion during the hypnotic trance. But with regard to the personalities thus evoked, as well as to those which are apparent during the waking life of the subject, it must be remarked that, in some cases, the so-called "control" is almost certainly different from any possible waking self of the individual concerned.

These facts would appear to point in the direction of two possibilities, either of which may occur in any particular case, while in some cases, perhaps, both may occur. It should first be remarked that to say that the individual associated with a given body has become a different person, is by no means the same as saying that he has become a different subject. It is meaningless to suppose that one subject can become another subject. But he may change his personality, so that the individual associated with the body may remain the same, though we might be quite justified in saying that he had become a different person. For the personality of a given subject is, so far as he himself is concerned, objective. It consists in that group of presentations which we call 
the "empirical self," that is, the self as conceived and imagined. Evidently our conception of ourselves will profoundly influence our actions, and it is in this way that our personality is manifested to others.

Now even in the most orderly minds, it cannot be said that the group of presentations forming the empirical self is co-ordinated to such an extent that it forms but a single unitary complex. The most prosaic of men has secret dreamings of himself as acting in various important and exalted capacities, and even in our daily routine we play many parts, the domestic and the social, for example, and conceive ourselves accordingly in the rôle of a father or a citizen ${ }^{1}$. Hence within that group which forms the empirical self, many complexes are distinguished. Normally these complexes are more or less linked by certain common elements, but at times it happens that one or more complexes become utterly distinct and "split-off" from the rest. This is the manifestation of a certain group of activities and tendencies which are in distinct opposition to all other activities. The latter may suceed in repressing the former, in which case they become mere tendencies, the corresponding images remaining subliminal. If, however, the reverse is the case, the abnormal activities may almost entirely displace the normal, so that the whole mode of living of the man will become different, and we have the spectacle of another personality manifested by the actions of the same subject. From what has been said, it would follow that "dissociated" personality is perhaps a better description of this kind of case, than "multiple" person-

${ }^{1}$ For a detailed account of this, see James Ward, Psychological Principles, pp. $3^{6} 7 \mathrm{ff}$. 
ality. If the new mode of action is so utterly different as to be devoid even of those common features which link an individual socially with the lives of others, we have positive insanity.

Cases of dissociated personality may thus be explained by supposing that certain groups of those subjective tendencies which are manifested by the complex of subliminal images forming the subliminal self, are not co-ordinated with those other groups, which, when they actualize, are manifested by the complex of images representing, when supraliminal, the normal empirical self. In general these distinct groups of tendencies are repressed by the inhibiting effects of normal activity ; but under suitable conditions they may be able to actualize. The images correspondingly presented as the new "self," will be accompanied by the associated motor presentations, and the mode of behaviour of the individual will be changed as a whole, in conformity with his altered personality. This sort of thing occurs in ordinary reverie (though the motor effects will generally be inhibited in this case), to a greater extent in persistent delusions, and worst of all in insanity. There is, therefore, strict continuity between the normal and the abnormal. In many cases of dissociated personality, however, even when the extremest differences of personality are manifested, there are no signs of insanity; that is, the new mode of behaviour conforms in all the usual ways to those principles on which social life is based, and which must therefore be common to all individuals if social order is to be maintained. The new group of activities is not, in such cases, completely " isolated " from all the rest, but is co-ordinated after the normal fashion 
with those fundamental forms of activity which make up the basis of the individual's common life in society.

With regard to the conditions under which a change of personality occurs, it may be remarked, in view of what has been said, that the reasons why it is possible to evoke secondary personalities during the hypnotic trance are now clear. For, as we saw when considering hypnotism, the nature of the trance is such as to render it easy to direct the activity of the subject in any desired way. In particular, if it be directed in one of those forms manifested by the secondary personality, the train of activity, once started in the absence of inhibiting factors, will continue, and the subject will behave accordingly. In this connection, the occurrence (probably invariably) of a certain period of unconsciousness before the change of personality is noteworthy, for it is evidently the sign of the presence of conditions which ensure the necessary withdrawal of attention from ordinary presentations, that is, the suspension of normal forms of activity.

The fact that bodily changes are sometimes the direct cause of those changes in the concept of self which constitute the mutation of personality, depends on two facts which are more or less connected. In the first place, from the objective point of view, it is on the original basis of bodily sensations that the concept of self is constructed, and, secondly, from the subjective point of view, since the dominant monad is to be regarded as immanent in the inferior monads composing his body, it follows that any profound changes in the latter will be accompanied by correspondingly profound effects on the former. But, on the whole, a change of personality 
produced in this way seems to be of a different type from changes of the kind considered previously. It does not appear to strike so deep, and generally it is of comparatively short duration. The effect produced is essentially similar, namely a changed concept of self, but the causes lie in different categories, and the changes they respectively produce in personality, though similar in essentials, differ very considerably in important details. The changes in personality due to bodily causes are mainly temperamental, on an emotional basis. But where the other causes we have considered are concerned, the changes are generally far more than temperamental. That deeper stratum of personality, to which we give the name "character," is also profoundly affected.

At the beginning of the discussion of multiple personality it was pointed out that the psychological facts seemed to lead to two possibilities ${ }^{1}$. Hitherto we have only considered one of these, namely when the subject remains the same although his personality changes. The second possibility is that in some cases it may indeed be a different subject with whom we have to deal. That is, it is perhaps possible in certain circumstances for the individual normally immanent in a given body to be displaced to a greater or less extent by another individual. Such an occurrence may be unusual, but he is rash who asserts it to be impossible. Indeed, those with the most expert knowledge of the subject agree in asserting that some cases cannot be accounted for by simply invoking a dissociated part of the personality of the subject concerned. Of the conditions under which this temporary invasion may take place we know

$$
{ }^{1} \text { Cf. p. } 277 \text { above. }
$$


little or nothing at present, although it is known how they may sometimes be brought about. For example, in the case of some "mediums," it is sufficient to put them into the trance condition for their bodies to be controlled, at any rate apparently, by other subjects. But discussion of this may be postponed till we come to consider mediumistic phenomena in general. In any case, occurrences of this kind are real examples of multiple personalities in association with the same body, and are quite different in type from the dissociated personalities of one subject. Both types may, however, occur in connection with one individual, the phenomena then becoming very complicated.

A classical example of what was probably a case of the above kind, is that known as the "Beauchamp" case. Miss Beauchamp manifested four distinct personalities, $B_{1}, B_{2}, B_{3}, B_{4}$ (so named by Dr Morton Prince). $B_{1}, B_{2}$, and $B_{4}$ seem to have been dissociated complexes belonging to the same subject-Miss Beauchamp; but all the evidence goes to show that $B_{3}$ ("Sally") was a different subject ${ }^{1}$. This is borne out in particular by the fact that $B_{1}, B_{2}$, and $B_{4}$ were finally fused by careful hypnotic treatment in which $B_{3}$ gave a helping hand. After this had been successfully accomplished $B_{3}$ gradually faded away to "where I came from," to use her own words.

We may conclude, then, that cases of alternating personality are of two types. In the first, only one subject is concerned, dissociated parts of his subliminal self becoming, for some reason or other, supraliminal. Here the change is on the objective side. In the second ${ }^{1}$ This opinion is held by Dr McDougall and others. 
type, the change is subjective, the same body being associated with more than one subject. Before we can satisfactorily examine occurrences fundamentally similar to these in general, it will be necessary to consider certain phenomena which appear intimately connected with the relation which links subject to subject.

(d) Abnormal Perception, Phantasms, and Telepathy. In the course of our investigations up to this point, we have been concerned, for the most part, with the individual and his activity. We now proceed to the consideration of phenomena of which the salient features depend not so much on the nature of the individual, but on the nature of the relations subsisting between different individuals. We pass, that is, from the subjective to the intersubjective, and as a consequence we shall now be particularly concerned with the universe as we have ultimately conceived it, namely as a plurality of individual subjects made one by a single, concrete, immanent entity, which is the ground of their interaction.

Abnormal perception of the type now referred to, consists in the perception of objects in circumstances in which they would not ordinarily be perceived. For example, a clairvoyant may see objects at a considerable distance which cannot be perceived by normal means at the place where he is situated; or he may see objects which are comparatively near at hand, but hidden. Clairvoyance can sometimes be induced in the hypnotic trance, though it is important to distinguish here between results which may be due to telepathy from the operators, and those which are genuinely clairvoyant. Careful experiment has shown that the latter element is undoubtedly present in many of the cases considered. 
The perception required is brought about during the trance by direction of the subject's attention to the appropriate object. Closely connected with this is the clairvoyance which undoubtedly sometimes occurs in dreams, during ordinary sleep; and as a connecting link between the two there is the case of the crystalgazer, where incipient hypnosis is induced by fixating the attention upon some particular object such as the traditional globe of crystal, whereupon abnormal perception frequently ensues. The important common characteristic exhibited in all the above conditions under which abnormal perception occurs, is the withdrawal of attention from the normal objects of perception. The attention is thus suspended, as it were, so that it is capable of being directed to impressions which would normally, perhaps, be comparatively too feeble to draw it definitely to themselves so that they occupied the focus of consciousness.

Abnormal perception must be distinguished from telepathy, which we shall discuss later. The former is manifested chiefly by the perception of objects similar in general nature to those ordinarily perceived. The latter is rather the "transmission" of an image or a thought from one subject to another. In the former the abnormal activity thus lies rather in the percipient; in the latter it would appear to lie more in the "transmitter," though no doubt there is generally something abnormal in the condition of the "receiver" also, at the time. Doubtless telepathy and abnormal perception have certain important features in common, and these may perhaps be indicated by those particular cases of the latter described variously as "phantasms" or 
"apparitions" of persons. It will accordingly be convenient to consider such appearances separately from the consideration of abnormal perception in general.

In attempting to explain satisfactorily the occurrence of abnormal perception of ordinary objects, there are, at the outset, two points to remark upon. Firstly, the difficulties generally felt, such as that of distance, are not real difficulties at all. For the ground of perception, whether normal or abnormal, is the interaction of the percipient subject with other subjects. Now subjects are not in space, so that difficulties such as those of distance are not really what they seem. No doubt certain spatial correlations of sense-data are the manifestation of the noumenal conditions necessary in general for that type of interaction between certain subjects which is the ground of perception. But it does not follow that these conditions are the only sufficient ones. For, secondly, as we saw, since the ground of the interaction of the many is one, it follows that each subject acts and is acted upon by every other. In the case of any given subject, the action of others upon him, who are, so far as their ordinary phenomenal manifestations are concerned, hidden or at a distance, is manifested by ultraliminal sense-impressions. If, for any reason, some of these become infraliminal, abnormal perception of distant or hidden objects occurs.

It was remarked above that abnormal perception was generally brought about by appropriate direction of the subject's attention when the latter is withdrawn from ordinary objects (as in sleep or in hypnotic trance). The impressions which are normally ultraliminal will then cross the threshold and enter the field of conscious- 
ness. Thus by partial or almost complete ${ }^{1}$ suspension of the subject's interaction with those subjects with whom he would normally interact most "intensely" under the given conditions, by the proper direction of his own activity, his interaction with those other subjects, who are generally manifested by ultraliminal impressions, becomes relatively and absolutely more intense, and the impressions enter the field of consciousness. Abnormal perception is thus simply the manifestation of the subject's interaction with certain other subjects under somewhat unusual conditions. The foregoing explanation would appear to demand a state similar to trance as a necessary condition in every case of abnormal perception; and the existence of this state is probably always a fact except, perhaps, in the perception of human apparitions. We shall see that in such cases, however, there is an additional factor concerned which has a considerable effect.

Before proceeding to the consideration of phantasms, one point of some interest in connection with abnormal perception deserves mention. This is the question whether action of this kind, of certain subjects on a given subject, is what is called "direct," or whether there are distinctive accompanying bodily conditions. Consideration of the phenomenal circumstances would seem to indicate with certainty that there are no peripheral conditions present analogous to those of normal perception. Whether there are cerebral conditions

${ }^{1}$ By reason of the unity of the world, there must always be some degree of interaction between any two subjects. In the case we are considering, impressions normally infraliminal and those normally ultraliminal will exchange places. 
similar to those normally present in perception is another matter. But, in any case, the question raised can be answered in a general way. There are two points. In the first place, it follows from the unity of the organism, the ground of which is the immanence of the dominant monad in the inferior monads of the body, that any interaction of the dominant monad with others must involve some specific action and reaction on the part of the inferior monads. It does not follow that the latter is a necessary condition of the interaction in which the dominant monad is concerned, but merely that it is a necessary accompaniment of that interaction while the dominant monad continues to exist in that particular association with its inferiors. In the second place, it is no less evident that this specific action and reaction of the body is, in the case of abnormal perception, different in important respects from that which occurs in normal perception. For while the general conditions of perception hold in both cases, the particular conditions (manifested by the particular objects perceived) in the case of clairvoyance or clairaudience exhibit unusual features, and the action of the body will be influenced by the latter as well as by the former. Perhaps the most probable conclusion from the scanty data available, is that in abnormal perception the conditions manifested by the cerebral state of the percipient are essentially similar to those which hold during normal perception, while the conditions manifested by his peripheral state are essentially different from the latter. But there is not at present enough evidence to point to a definite conclusion.

Coming now to the question of human apparitions, 
we have two distinct types of occurrence reported, namely phantasms of the living, and phantasms of the dead. In the case of the former, it would seem that it is sometimes possible for the agent to cause a phantasm of himself to appear to some percipient by sheer volition. There are two points to notice here. Firstly, in the cases reported, the apparition is not generally perceived at the time of the agent's volition, but later on when the agent is sleeping, or in a state of incipient sleep; and even when the apparition occurs in rare cases at the time of volition, it is not nearly so vivid as when it occurs later. Secondly, the percipient is usually (though not necessarily) closely connected by kinship or acquaintance with the agent; and it may here be remarked that in such cases apparitions have been reported when the agent was in an unusually intense state of emotion, though apparently no definite volition to appear to the percipient existed. Thus we have as the main features in phantasms of the living: ( I) A definite volition, or a state of intense emotion but apparently with no definite volition, on the part of the agent; (2) the apparition generally occurs after the volition or emotion, during sleep or incipient sleep of the agent; (3) a close connection usually exists between percipient and agent. The last seems to be invariably the case when the ground of the apparition is intense emotion on the part of the agent.

In setting out to explain phenomena of this kind, we must first of all notice the important feature in which they differ from abnormal perception of inanimate objects. In the case of the latter, the percipient's attention is definitely withdrawn from normal impressions. 
It is his activity (directed in abnormal forms), which is the initiating cause of the occurrence. We can hardly suppose that the activity of those subjects of which the objects perceived are the manifestation, is an efficient factor in initiating the abnormal perception, though of course the latter depends on it. But in the perception of human apparitions it is evidently the activity of the agent which brings about the unusual occurrence, and not that of the percipient. For the attention of the latter is not in general withdrawn from normal impressions when he perceives the apparition. Hence, so far as the percipient is concerned, in ordinary clairvoyance the initiation is subjective, but in the perception of phantasms it is objective, its ground being the activity of the agent, which causes impressions to enter the field of consciousness of the percipient which would normally have remained ultraliminal.

The activity of the agent which gives rise to the phantasm is in some cases consciously directed towards that end, in others not. Where it is a definite volition, we noted above that the phantasm generally follows the volition after a certain period, the time of its occurrence coinciding with sleep or incipient sleep on the part of the agent. This may be explained as follows: At the time of the volition, if the agent is fully awake, his activity is necessarily directed in many other ways also. This is the case even when his concentration on the volition is very considerable. The inhibition will therefore generally be sufficient to prevent the definite outcome of the volition, namely the apparition it is purposed to bring about. When the volition ceases consciously, it remains as a strong tendency, the image-

R. S. P. 
complex, which is its immediate object, becoming subliminal. With the gradual cessation of ordinary activity as sleep comes on, the tendency passes into actuality, the image-complex rising above the threshold, and in the absence of the previous inhibiting factors the desired result occurs. Evidently in some cases the inhibiting factors may not be sufficient entirely to prevent the outcome of the volition even at the time of its inception, and the phantasm will appear simultaneously with the occurrence of the volition. This does, in fact, sometimes happen, but, as might be expected, the phantasm is not then so clear as when it occurs after the volition under the conditions considered above.

When a phantasm appears in this way as a consequence of a definite volition, there is apparently no necessity for any particular connection (such as ties of relationship or close acquaintance) between agent and percipient. The place at which, and the person or persons to whom, the phantasm is to appear, simply form part of the object of the agent's volition. But in the second type of cases, namely when some abnormal emotional condition (and not a definite volition) of the agent is the ground of the appearance of the phantasm to the percipient, the latter, in all recorded cases, seems to be connected in some especial way with the agent. This condition replaces the condition in the former type of cases which required that the desired percipient should form part of the object of the agent's volition. There is now no definite volition to appear on the part of the agent, but, on the other hand, the percipient is a person of whom he would naturally think under the given conditions, namely someone closely connected 
with him who would thus be much affected by anything which affected him (the agent) considerably.

The ground of phantasms of this type is not far to seek. It lies ultimately in the fact that the experience of each individual consists in bringing himself into harmonious adaptation with the individuals composing his environment, by continuous interaction with the latter, thus setting up a more and more sympathetic rapport between himself and them, in virtue of which he readily responds to changes in them. This responsiveness to changes in others reaches its highest degree when those others are human beings closely connected by the ties of family or social intercourse with the individual concerned. Even when separated from them, so that the impressions which manifest them to him are ultraliminal, the individual's responsiveness to changes in them will retain relatively its high degree. Hence he will sometimes perceive a phantasm when a close friend or relation is undergoing intense emotion, for example, for the activity consequent upon this emotion may be sufficient to cause the corresponding ultraliminal impression in the percipient's object of experience to enter the field of consciousness. Other conditions must, however, be taken account of, for otherwise such apparitions would be far more frequent than they actually are. Evidently the mental state of the percipient at the time will be favourable or otherwise according as his attention is actively engaged by other matters or not $^{1}$. As to further effective

${ }^{1}$ Sleep on the part of the percipient would thus, other things being equal, be a particularly favourable condition; and it is noteworthy that phantasms recorded as appearing during sleep are 
conditions ${ }^{1}$, there is not at present enough data available to form any definite conclusions.

Phantasms of the dead are on a somewhat different level from those of the living, for the data available will be furnished from one side only, namely that of the percipient. In the case of apparitions of living people it is generally possible to discover what the agent was doing or thinking at or about the time he appeared to the percipient. But this is of necessity impossible in the case of one who is dead.

This drawback is, however, to some extent counterbalanced by the fact that phantasms of the dead occur for the most part ${ }^{2}$ immediately after death, or within a short period. Not only is this connection between the actual death and the apparition of evidential value, but a knowledge of the circumstances of the former may provide, in some cases, valuable information as to the conditions under which such apparitions occur. The latter seem to fall into two classes. In the first there is some definite and generally close connection between the (apparent) agent and the percipient. In the second no such connection exists; it is replaced by a connection between the agent and the place at which his apparition is perceived. Anyone may happen to be the percipient.

especially vivid. There is here, however, a possibility of telepathic action in many cases. (See later.)

${ }^{1}$ It is possible, for instance, that ties of blood may alone be sufficient to constitute some basis for the necessary degree of sympathetic response in favourable circumstances.

2 The occurrence of apparitions a considerable time after death is reported in some rare cases, but the phantom then seems to be much fainter than when its appearance follows death after a short interval only. 
These cases of so-called "hauntings" are neither so frequent nor so well substantiated as those of the first class. In the case of the latter there is generally one percipient only, but when the "ghost" haunts a certain locality there may be many percipients at the same or at different times.

Phantasms occurring after death do not necessarily imply the continued existence out of the body of the person perceived, for the latter is not a temporal entity although his manifestation is. We can only say that the sense-data of the percipient which constitute the apparition of the given individual are temporally after those which constitute, for other people, the death of that individual. There is no way of correlating either of these two sets of sense-data with those of the individual who has died, after such a fashion that it might be significantly said that the occurrence of one set of sensedata after the other is evidence of the existence of the individual out of his body ${ }^{1}$. But at least it may be said that there is a certain presumption created in favour of such a conclusion; and, as we have seen ${ }^{2}$, there is no valid reason for doubting the existence of subjects out of the association with their present body. They would still continue to act, then, upon other subjects living in

${ }^{1}$ In the case of an apparition which is merely visible, there is no way of establishing that correlation between the sense-data of the percipient and those of the agent, which is necessary to indicate the existence of the latter out of his former body [see the essay on "Immortality," Section II]. When a communication is made by the agent, however, to the effect that he has survived death, evidence of identity must be considered on the same basis as that on which we normally identify an individual by our sense-data.

${ }^{2}$ See the previous essay, Section VI. 
the body, being manifested, for the most part, by ultraliminal impressions. In certain circumstances the latter might become infraliminal, and the connection between percipient and agent, frequently, reported in such cases, is explained in the same way as the similar connection observed in the case of phantasms of the living. There is no real evidence for "survival," however, unless a definite communication is made by the agent which is original and sufficient to establish his identity. Such a communication is rarely made in the case of apparitions of the kind we have been considering.

With regard to what are known as "hauntings" by apparitions, of particular places, the evidence adduced is very meagre and incoherent, and until fuller substantiation is obtainable there is neither demand for, nor possibility of, an explanation. Suffice it to say here that reported cases are of two kinds. In one a definite routine is repeated by the apparition on each occasion, in the other there seems to be an attempt at communication on the part of the apparent agent. Of the first it may be remarked that there is here, perhaps, something analogous to certain phantasms of the living. In the latter we have a manifestation of the agent with unusual spatial correlations-manifestation at an abnormal distance-in the former we have possibly an analogous manifestation but with unusual temporal correlationsmanifestation after an abnormal interval of time. The agent not being himself in space and time, although his manifestations are, there is nothing inherently impossible or contradictory in such a supposition. Invariable connection with a particular locality would then present no difficulty. But to account for the fact that in 
such cases there is apparently no necessary connection between agent and percipient, it certainly seems necessary to suppose that some individuals are so constituted that they are peculiarly affected by abnormalities of this kind, while others are not; and, in fact, in cases of haunting as reported, it is frequently stated that the apparition is seen by some persons but not by others.

Where there seems to be an attempt on the part of the apparent agent to establish communication, the case is similar to that of those phantasms of the dead first considered. The temporal correlations of the apparition with the experience of the agent himself (assuming the reality and identity of the latter) would then not be unusual; and the connection with a particular place is simply accounted for by supposing that the interests of disembodied subjects, so far as they continue to refer to this life, will centre round the same localities as during the bodily life of the subjects concerned. But the facts, if truly reported, would still demand some special peculiarity in the constitution of the percipient, as in the previous type of case. This, of course, merges into the general problem of mediumship.

In phantasms both of the living and of the dead, it is important to distinguish between the presentation of a true sensory impression, and that of a vivid image "transmitted" by telepathic action. Hitherto we have confined our attention to the former. Now it is reasonable to suppose that the sensory manifestation of a disembodied subject would be similar in a general way to that of the same subject while still in the body. For we saw ${ }^{1}$ that the manifestation of another living

${ }^{1}$ Cf. the previous essay, Section IV. 
person $A$ to us, is not simply the appearance of the group of inferior monads $B$ composing his body, but of the complex $A$-immanent-in- $B$, the whole form of the appearance being largely shaped by the immanence of the dominant monad. In some phantasms of the dead the appearance perceived is comparatively vague, though agreeing in its general features with the former appearance of the individual. But on other occasions the appearance exhibits such details as, for example, the clothing formerly worn. In such cases, where we also have grounds for supposing that we are not dealing simply with manifestation after an unusual temporal interval but that there subsist the normal correlations of the appearance with the experience of the agent, there can evidently not be true sensory perception involved. Telepathic action may, however, afford the necessary explanation.

But before passing to the consideration of the latter, it is necessary to say a word on the question sometimes asked: "Are phantasms objective?" Clearly the question as it stands is meaningless. A phantasm is an object presented to a subject, although there may be a breakdown of certain normal correlations of sensedata. Doubtless it is to something of this kind that the question is meant to refer. In particular, it is said that phantasms are "intangible." That is, if we put our hand (say) where the phantasm appears (visually) to be, we feel nothing. This is not remarkable in view of the fact previously noted, that in the perception of such apparitions the phenomenal circumstances which constitute the peripheral conditions of normal perception are undoubtedly absent. Thus in the case of phantasms, 
albeit visual, the eye would be as little affected as the hand. The attempt to get the correlated tactual impressions by normal motions of the periphery is not strictly comparable with the seeing of the phantasm, for this is abnormal, and not correlated with the usual peripheral conditions of the eye. The true analogue of a visual phantasm which could not be touched by moving the hand would be a tactual phantasm which could not be seen by moving the eye, nor detected by the other sense-organs. The occurrence of such phantom touches has, indeed, been reported on many occasions, but they certainly do not seem to be so frequent nor so well substantiated as are visual apparitions.

We now pass to the consideration of telepathy or "thought-transference." This must be carefully distinguished from clairvoyance and other forms of abnormal perception. In cases of the latter, the object presented to the percipient may be regarded as a genuine sensory impression which is an appearance of the agent. In telepathy, however, the object presented to the recipient is not an appearance of the agent, but an image similar to an impression or image presented to the agent. There is thus a distinct difference between the two processes. In the case of telepathy the object presented may indeed sometimes resemble the appearance of the agent, but only when the latter is thinking of himself. There is little doubt that many cases of phantasms such as those we have been considering, are really due to telepathy of this kind and not to what may strictly be called the abnormal perception of an appearance of the agent.

Briefly, then, the facts are as follows: Telepathy is the occurrence to one individual (the recipient) of a 
certain presentation, either simultaneously with, or shortly after, the occurrence of a similar presentation to another individual (the agent). As regards the recipient, the results are best when he is in a passive waking state or in a hypnotic trance, but (if waking) his interest must be kept fresh. As regards the agent, the transmission may be deliberate or spontaneous, no special effort to transmit is necessary, and, in fact, too great a concentration on the desired result seems to inhibit its occurrence almost completely. The recipient appears to get an image essentially similar to the presentation to the agent. Thus if the latter has a visual impression the former speaks of "seeing" the image. In certain cases of hypnotic trance there is a truly remarkable community of presentations (of all kinds) between agent and recipient. Sometimes, however, it would seem that it is not the actual image of the impression which is transmitted, but a closely associated image to which the agent may also be attending, such as the name of the object. This is shown by the fact that the recipient will mistake (say) the eight of hearts sometimes for the eight of diamonds (similarity of visual presentation), sometimes for the ace of hearts (similarity of auditory presentation of name). Frequently there is some delay in the emergence of the presentation in the recipient's field of consciousness. In some cases the delay is considerable, and perhaps in every case there is a period of lag, though generally too short to be observed. Finally, it may be noted that the effect of the distance between agent and percipient is little or none; the distance may be very considerable without appreciable diminution of the effect. In 
particular, it is sometimes possible to induce trance at a distance.

Evidently telepathy is explained in principle by the immanence of a single concrete entity in the individuals composing the world, which is the ground of the latter. As we have seen, such an entity must be postulated to explain any interaction between individuals. But this alone will not suffice to account for the particular form which telepathy takes. Both in telepathy and in perception (whether normal or abnormal) the activity of one individual influences that of another, but in the case of the latter what is perceived is an appearance of the agent, that is we have reciprocity of action between agent and percipient. In the case of telepathy, on the other hand, the object presented to the recipient is an image similar to an image or impression presented to the agent, so that we have here rather community of action between agent and recipient. In perception the activities of the two individuals are of different forms (for the presented objects differ), though each form is determined by the interaction between the individuals. But in telepathy the form of the activity is the same in the case of each of the individuals concerned, for the presented objects are similar.

Apparently, therefore, the precise relation between the two subjects in telepathy must differ from that which holds in abnormal perception. Can the two sets of relations be reduced in any way to one? Only by regarding all cases of abnormal perception as particular examples of telepathy, and not as cases of true sensory perception at all. We should then have to suppose that the ground of an apparition of the agent was never the 
agent himself, but always an image of him presented to himself. As we have said above, some apparitions are almost certainly engendered in this way. But it does not seem possible thus to explain away all abnormal perception. For example, in cases of the clairvoyant discovery of so-called "inanimate" objects, we can hardly suppose that an image of the latter is presented to the subjects of which they are the appearance, and that this is the ground of the clairvoyance. It is therefore impossible to reduce abnormal perception and telepathy to two particular examples of what is fundamentally one and the same process.

We cannot hope to resolve the difficulty which thus arises, without enquiring somewhat further into the consequences which follow from the view we have adopted of the constitution of reality. The known facts about telepathy are not sufficient to warrant the definite assertion of a solution of the problem; but the direction in which a satisfactory solution is probably to be sought may be provisionally outlined.

Reality we have supposed to be constituted by a plurality of true individuals welded into one universe by the immanence in them of a single concrete entity. The existence of the Many consists in their interaction with one another, but the condition of the possibility of this interaction is the immanence of the One. Hence the Many are not self-existent, but neither are they merged in the One so as to lose all individuality. Rather is there in the nature of each an element of individuality and also an element of universality. The latter is a necessary ground of the activity in which the assertion of individuality consists. It follows, therefore, that the 
activity of a given subject may be conceived under two aspects : as an assertion of self as distinct from others, and as an expression of an impulse in that unity of which self and others alike are members. These two aspects are complementary; in particular cases comparative stress may be laid upon either. All activity is thus the joint assertion both of the distinctness of individuals of a plurality, and of their common membership of a unity; it will therefore be constituted by both reciprocity and community of action.

It is impossible, of course, to express these facts satisfactorily. We are here dealing with what is essentially subjective, so that no analogy from the objective can adequately demonstrate all that is implied. One particular case is of some help, however, namely the relation of the individual to his body. I can see my body, but I can also act with and through it. In the former case there is reciprocity of action between myself and the monads constituting part of my body. In the latter case there is community of action. The organism is therefore a veritable microcosm. For thus it is in the macrocosm we call the Universe. The ground of perception (whether normal or abnormal) is reciprocity of action between individuals; the ground of telepathy is community of action, manifested in the presentation of a similar object to each of the individuals concerned. The comparative degree in which these complementary functions enter into the activity of a given individual will depend on the attitude he takes up-whether he asserts himself as an individual distinct from others by his concentrated efforts, or whether he takes a more passive attitude, and is swayed by impulse 
(as we say), not only in deed, but in thought and in imagination.

In this way those attitudes of agent and recipient respectively, which appear to be necessary conditions of telepathy, are explained. Not only should the recipient adopt a passive state, but the agent also should refrain from any special effort. It is plain, too, that telepathy will occur not only when the conditions are deliberately set, but spontaneously, though in the latter case it may frequently not be observed. The occurrence of spontaneous telepathy in this way is known to be a fact.

In telepathy we are concerned with a relation which is purely inter-subjective. This relation is manifested by the presentation of similar objects to two different individuals, that is, it is manifested by a certain correlation of the sense-data ${ }^{1}$ of one individual with those of another. Hence we should not expect the correlated sense-data to exhibit the same connections as those between correlated sense-data all of which form part of the object of experience of the same individual. The inverse square law, for example, which is based on certain correlations that may be observed in the sense-data of any single individual, will not necessarily hold in the case of a correlation of sense-data in which there must always be more than one individual concerned. Hence it is not surprising that telepathy exhibits none of those diminishing effects with distance which are found to occur in the case of ordinary material radiation, and this quite apart from the fact that subjects are not in space. There is, indeed, but a very remote analogy be-

1 "Sense-data" is here used in a wide meaning as including the images of sensory impressions. 
tween telepathy and the transmission of the various forms of radiant energy.

Finally, the delay which sometimes occurs in the emergence of the image into the recipient's field of consciousness may be accounted for by the inhibiting effects of his other activities. In this case, the activity of the agent will be accompanied by the setting up of a tendency on the part of the recipient to act in the same way. Even should this tendency not actualize, owing to inhibiting factors, it will remain as a tendency to act in a particular way, and when the inhibition is absent, it will actualize. On the objective side, this is manifested by the presence in the recipient's object of experience, at the time the given impression is presented to the agent, of a subliminal image, which is prevented from rising above the threshold by the intensity of the normal images and impressions which already hold the field. Later on, when the recipient's attention is withdrawn from these distracting presentations, the subliminal image may rise above the threshold.

If telepathy is thus the manifestation of the tendency on the part of individuals of the plurality to act, in virtue of their immanent unity, in the same way, why, it may be asked, is it not observed more commonly? Now, in the first place, the form of the activity of any given subject, is the joint product of his own individuality and of his interaction (in its aspects both of community and of reciprocity) with other subjects. Hence it is evident that unless an appropriate attitude is taken up by the two particular individuals between whom telepathy is desired, there is little likelihood that their activities will take forms manifested by presentations 
which are recognizably similar. But, in the second place, it is quite possible that in spite of this, telepathy occurs far more commonly than is actually observed. How many of the images and thoughts which spring unbidden to our minds may not be the manifestations of its working, although we may not, by the circumstances of the case, be able to verify that this is so? The common impulses which seem under certain conditions to govern the actions of members of a crowd, for example, may be due largely to telepathy, the circumstances being appropriate.

Before proceeding to our next topic, it may be repeated that the foregoing does not profess to give a complete solution of the problem of telepathy. Its aim is purely tentative, which is all it could hope to be in view of the present state of our knowledge of the subject. But it may at least serve to indicate the direction in which an explanation is perhaps most likely to be found, and the general form that such an explanation will take.

(e) Automatic Writing and the Problem of Mediumship. Communications purporting to come from discarnate agents, are generally made through the instrumentality of a particular type of person, to whom the somewhat unsatisfactory name of "medium" has been given. The problem of the nature of the process involved in mediumship, is best approached by the consideration of the simplest manifestation of the latter, namely automatic writing.

In automatic writing, the hands of the medium are placed on a planchette or ouija board, or a pencil is simply held passively between the fingers, whereupon movement takes place and messages are written out or 
indicated by letters, without any conscious volition on the part of the medium. During the process the latter may be in a condition of normal wakefulness or in a condition of trance; and, if waking, his thoughts and conversation may be directed to quite different matters without influencing or interfering with the writing. The last fact marks an important distinction between the two sets of conditions (trance or wakefulness) under which automatic writing may take place. The difference is emphasized by the fact that during trance automatic speech also frequently occurs, whereas this is never the case during the normal wakefulness of the medium.

The writing or speech sometimes conveys true information of distant events occurring at the time which could not possibly be known through any normal means by those present. In such cases, telepathy between the latter and the medium is ruled out. Again messages come at different times through the same medium, which are evidently due to different personalities, although this does not imply necessarily that more than one subject (the medium himself perhaps) is at work ${ }^{1}$; in any case, there are indications of the influence of the medium's personality in various ways. Conversely, in messages coming through different mediums, there is sometimes evidence, after the elimination of minor differences due to the influence of the personalities of the various mediums, that the same personality (and therefore almost certainly the same subject) has inspired the messages in each case $^{2}$.

${ }^{1}$ Cf. Sub-section (c), supra.

${ }^{2}$ Cross-correspondence supplies evidence of this kind, of a particularly valuable nature.

R. S. P. 
At the commencement of a sitting, before the communication gets into full swing, the entranced medium generally speaks or writes a few words of sheer nonsense. This is probably comparable with the chaotic array of thoughts and images which ordinarily flit before the mind during the transition period between sleep and wakefulness, and affords valuable indications of the real nature of the process we are examining. In this process, it appears necessary to make a distinction between "communicators" and "controls," as they are called. There is reason to believe that a particular medium is almost invariably controlled by the same agent, who is apparently a different subject from the medium and who acts as intermediary for the various communicators from whom the messages ostensibly come.

Assuming, without further discussion for the moment, that in the cases we have been considering there are really, and not merely apparently, subjects different from the medium himself, who "control" and communicate through him, there are certain facts which call for remark. Chief among these is the fact that the controls have to work, to all appearances, under considerable, and sometimes very great, difficulties. This seems to be mainly due to two causes. In the first place, the medium can never be an entirely passive instrument; and, secondly, it is very hard, and perhaps impossible generally, to transcend certain limitations imposed by the constitution of the particular mediumistic organism ("body" and "mind") as a whole. Examples of this are to be found in the differences which occur when the same control or the same communicator works through different mediums. On whatever plane the thoughts 
embodied in the messages may be, the particular mode of expression adopted largely depends on, and is limited by, the constitution of the medium. A point may be noted here, however, which may prove later on to have some bearing on this question, and that is that in some of those comparatively rare cases when the medium retains a certain memory of what has happened to him during his trance, he speaks of having been translated to another realm inhabited by beings in many ways superior to those of earth. If there is such a realm and such beings, their onward progress perhaps results in a rapid loosening of the bonds which still connect them with this world, for it is certainly true that messages from the same communicator become more and more vague and fragmentary, after a certain point, as time goes on.

Before attempting to explain these mysterious facts, a word may be said on the question as to what special peculiarity is involved in the nature of a medium. This is a difficult question to answer, for in spite of detailed investigations it has not yet been possible to discover, amongst the endless diversities of nature exhibited by various mediums, just what are the specific necessary qualities common to all. An examination of results, however, makes one thing clear, namely that mediums are always essentially "suggestible" subjects, and perhaps the quality thus found to be common to them all, may turn out to be the most necessary one. This is indicated by the fact that mediumship certainly exhibits features analogous to the "rapport" which holds between operator and subject in the ordinary hypnotic trance.

In the early stages of development of the faculty, 


\section{Subconsciousness and Abnormal Phenomena}

the medium is usually confined to a passive automatism, but as the development progresses, the attitude taken up becomes more active, culminating frequently in such powers as the clairvoyance and clairaudience of apparitions. Some mediums also have the power of "psychometrizing" as it is called, that is, of intuiting from the perception of an inanimate object, the nature and circumstances of its owner.

A necessary preliminary to the explanation of the processes involved in mediumship, is the attempt to obtain a definite answer to the question as to whether the movements of the medium may be taken in any case at all as manifesting the activity of a subject different from the medium himself. The existence of waking, as distinct from entranced, mediumship fortunately enables us to come to a decisive conclusion on this question. For in ordinary automatic writing, for example, the medium may at the same time carry on a totally distinct train of thought or action. In extreme cases, the medium will maintain a spoken conversation with one person while his hand writes replies to the whispered questions of another person, the whispers not being perceived by the medium himself. If the writing obtained were always sheer nonsense, or, if making sense, consisted simply in the mechanical repetition of information known to the medium, it might be possible to maintain that the planchette or pencil gave certain latent tendencies in the medium an opportunity to actualize while his attention is directed elsewhere, the images which, in rising above the threshold, manifest the actualization of these tendencies, not occupying his focus of attention. But this is far from being the 
case. The writing frequently manifests the operation of a distinct and original line of thought (as when questions are relevantly answered) which could not possibly be carried on automatically without the definite direction of attention to it; yet at the same time the medium's attention may be clearly directed elsewhere. Hence in such cases, two distinct foci of attention must be involved, and therefore two distinct subjects-not merely two personalities. This conclusion appears quite incontrovertible. The most rapid oscillation of attention could hardly account for the facts, and there would still remain the difficulty that, although fully awake, the medium was conscious of the carrying on of one line of thought, but quite unconscious of the carrying on of the other.

In such cases, then, there only remains the question as to whether the agent is distinct from the organism of the medium or whether he is one of the inferior monads composing the body of the latter. The second alternative seems unlikely, for it is often quite evident that the agent is at a level of experience at least as high as that of the medium. Now it is very doubtful, in view of the difference in their general functions, whether an inferior monad could ever approach the level of the dominant to whose body he belongs. The kind of social intercourse on which the development of the dominant monad is largely based must necessarily be absent in the case of the inferior monad; and the most conceivable possibility would be that certain inferior monads might perhaps be so situated as to be able to witness unobserved (for the most part) the life of the 


\section{Subconsciousness and Abnormal Phenomena}

dominant. In any case it would be unwise to make a dogmatic assertion on the point. It is better to be content with saying that it seems highly improbable that all the communications received by automatic writing can be attributed to the inferior monads of the medium's body.

Leaving for the moment the investigation of the modus operandi involved in automatic writing and mediumship generally, we may first briefly consider what is implied in the facts of entranced mediumship. There is no doubt that in this case the various apparent agents may simply be different personalities of the same subject-the medium. This will be evident when what was said above in connection with the hypnotic trance and with multiple personality is remembered. For, owing to the entranced condition of the medium, we have here no evidence of the existence of more than one focus of attention. Nor would it be necessary for more than one subliminal personality to be concerned. This personality, as the apparent agent, would describe himself as the "control," the visits of various communicators being pure invention on his part, for it is well known that the moral standards of the subliminal personalities differ considerably from those of the normal personality; the medium, indeed, on awaking, would remember nothing of what had happened. On the other hand, it is just as possible a conclusion that in some cases, at any rate, the communications are really due to other subjects, and this is rendered more probable by the facts of waking mediumship. The question is, however, one which can only be settled by internal evidence, that is, by examination of the messages actually received. 
The results of such an examination give probability to the alternative that agents other than the medium are sometimes involved. For not only do some of the personalities at work differ from any even remotely conceivable personality of the medium, but (as was mentioned before) true information is sometimes conveyed which could not possibly be known by normal means to the medium or to the other sitters present; and frequently this information is of such a nature as to strain any hypothesis of telepathy or clairvoyance on the part of the medium to a wellnigh inconceivable extent. Finally, there is the elaborate evidence of identity obtained by cross-correspondence in the messages received through different mediums.

Assuming, then, that in some cases at any rate, the control and the communicators are really different subjects from the medium, we have now to consider the difficult question of their modus operandi. It seems possible that more than one method might be adopted. The similarity, pointed out above, between the mediumistic and the hypnotic trances, leads to the supposition that sometimes, perhaps, communications may be made by actually hypnotizing the medium, the control being the operator. Owing to the withdrawal of the medium's attention from those presentations which are normally in the field of consciousness, ultraliminal impressions would be enabled to enter the latter, and hence the control, manifesting himself to the medium, might cause the latter to write or speak as desired, by direct suggestion, or perhaps by telepathy. In the latter case, a definite objective manifestation of the control to the medium would be unnecessary. 


\section{2 Subconsciousness and Abnormal Phenomena}

That hypnotic action is the mode of operation sometimes adopted by the control, would seem to be borne out by the fact, remarked on previously, that the one feature which appears to be common to all mediums is that of suggestibility. The control, as operator, does not immediately direct the movements of the medium in these circumstances. They are directed by the medium himself, acting in accordance with the suggestions of the control. Consequently we might expect to find the communications tinged throughout with traces of the medium's personality. But it is not, at first sight, so clear why the mode of expression employed should be limited for the most part by the capacities of the medium. For why should not the messages be dictated, as it were, by the control word by word, and mechanically repeated by the hypnotized medium? Probably the reason is to be found in the nature of the manifestation of the control to the medium. We do not know what form this takes. It may necessarily be such, that only a comparatively general notion of the ideas it is desired to convey can be imparted to the medium, who accordingly expresses them in his own way. It certainly seems very probable that, owing to the difference in the nature of the conditions involved, the control cannot impart ideas to the medium by any instruments as precise as those employed in ordinary social intercourse, such as language, for example ${ }^{1}$. On the other hand, if

1 With practice on the part of the medium, however, the manifestation of the control, and consequently the meaning intended to be conveyed, may become clearer to him. This probably explains the gradual development, mentioned previously, of a more active attitude on the medium's part, frequently culminating in the clairvoyance of apparitions. 
telepathy be the mode of operation adopted, the case is still clearer. For, as we saw when considering telepathy, the recipient's presentation is frequently not of exactly the same form as that of the agent, but of one closely associated with it-e.g., essentially the same thought may be embodied in different images in the two cases. The recipient's activity may not be capable of taking exactly the same form as that of the agent. Hence in mediumship, the transmission of a presentational complex from the control will, in such cases, enter the medium's field of consciousness embodied in images essentially akin to those to which the medium's attention is normally directed, but by no means necessarily similar in quality to those present to the control at the time.

Taking all the recorded facts into account, it would appear certain that hypnotic action cannot be the only mode of control adopted. It is necessarily ruled out in the case of waking mediumship, for example. We can only suppose, then, that in cases of this kind (as well as in some trance mediumship perhaps) the body of the medium, or part of it, is directly controlled by another subject. How this is possible is indeed a problem. For we do not even know the phenomenal conditions under which a dominant monad may cease to be immanent in his body, except in the case of those constituting death ${ }^{1}$.

${ }^{1}$ It is possible that, in terms of experience, the mode of action necessary to ensure withdrawal of the dominant monad from his body, is the withdrawal of his attention from all bodily sensations, together with a temporary cessation of perception and the volition of movements. This is partially accomplished in sleep, but it would evidently be exceedingly difficult to carry out completely. It is noteworthy, however, that certain African natives seem to be able to lie down and simply die, although they have no bodily ailment, just as 


\section{I4 Subconsciousness and Abnormal Phenomena}

Moreover, in automatic writing and speech, a strange subject is apparently associated with part of the body in the same way as its dominant monad. When this happens during trance, it is possible that, while the control is in possession of the body, the dominant monad (i.e., the medium himself) temporarily withdraws. Colour is lent to this suggestion by the accounts given by some mediums of their transference to another sphere during trance ${ }^{1}$. In waking mediumship, however, this is ruled out, and as hypnotic action is also out of the question here, we are apparently confronted by the strange fact of two different dominant monads controlling different parts of the body simultaneously. There is no great difficulty involved in the idea itself, however; for, as we saw elsewhere, owing to a certain decentralization of bodily functions ${ }^{2}$, a considerable part of the latter habitually go on without any attention on the part of the dominant monad. This being the case, it is not difficult to conceive that another subject might take control of these functions in order to direct them a particular way, while the dominant monad's attention is turned elsewhere. If this be so, the association of this other subject with the parts of the body concerned cannot be very binding, for, at any rate in waking mediumship, the medium can resume control of the parts affected without any difficulty. In any case, however, the association and co-operation of the inferior monads of the body with the dominant monad normally immanent in ordinary people lie down and sleep. As to the converse process, namely the "entering" of a body, we can of course form no conception.

${ }^{1}$ Cf. supra, p. 92.

${ }^{2}$ Cf. the previous essay, Section IV. 
it, is so intimate that any intruding subject would necessarily work under great difficulties until he became habituated, and expressive actions, though initiated by him, would be reminiscent of the body when under the control of its normal dominant. For the body is not a merely passive instrument. The forms of its activity are largely shaped by the nature of the particular subject immanent in it; nor would these forms readily change at the intrusion of another subject. If hopeless dislocation, leading to no intelligible expression ${ }^{1}$, is to be avoided, the stranger must work by directing the activity of the body in habitual ways, as far as possible, and must seek to express himself simply by that combination of available actions which approaches the desired result most nearly. It is evident from such considerations, that the difficulties under which the control generally works, even so far as we can judge, must be immense, and there are almost certainly other difficulties of which we know nothing.

The only alternative to assuming that the control sometimes operates immanently through the inferior monads of the medium's body, would be to suppose that in all cases of the kind the control acts hypnotically on the latter by direct suggestion. But, quite apart from the almost insuperable difficulties involved in any attempt to hypnotize subjects at a comparatively low level of experience, this hypothesis would bestrained to breaking

1 The nonsensical expressions which often occur at the beginning of a sitting are probably due partly to the medium himself, as he passes through the transition stage between waking and trance, and partly to the fact that the invisible operator has not yet gained adequate control of the medium's body. 


\section{I6 Subconsciousness and Abnormal Phenomena}

point by the occurrence of what seem to be genuine "possessions" in certain cases of multiple personality ${ }^{2}$ Now if a foreign subject may be immanent in the body in such cases, there is no reason why the same should not occur in ordinary mediumship. Certainly there is no a priori objection against it.

The residual problem in this branch of investigation is, then, to determine how, and under what conditions, it is possible for different subjects to be associated with the same body. This problem is, in fact, the same in type as that of birth, namely, how does any particular dominant monad become associated with a particular set of inferiors? A further discussion of the point would take us beyond our present limits. Before closing this section, however, it may be remarked that the fact that a particular medium is generally controlled by the same agent, is probably of considerable significance. In the hypnotic mode of control this may be explained by the well-known fact that hypnotic subjects are peculiarly susceptible to the influence of certain operators. In cases where the immanent mode of control is employed, on the other hand, it will evidently be the case that certain subjects will be able to work through a particular body far more easily than others, in fact the number that can work with any success through a body which has necessarily been drilled in certain ways by the immanence of a unique individual, will be very limited indeed. Here we must leave the question, however, passing finally to the consideration of a strange class of phenomena, which seem to involve the presence of a medium, but in a somewhat different way.

${ }^{1}$ Cf. supra, Sub-section $(c)$. 
(f) Levitation and allied Physical Phenomena. The peculiar phenomena which may be grouped under this heading, appear to require, as a necessary condition of their occurrence, the presence of a particular person in each case, to whom the name of "medium" is accordingly given. They differ from the last class of phenomena, however, in that the part played by the medium is not directly observable. The function of the medium is, indeed, apparently analogous to that of the catalytic agent in certain chemical reactions. Although his presence is necessary, no special mode of behaviour on his part seems, in general, to be required ${ }^{1}$. The physical objects affected are not in contact with him, nor is his attention necessarily directed to them particularly.

Perhaps the most remarkable of the phenomena manifested in this way, is the levitation of the medium's body, or of other objects, without visible or tangible cause. Phenomena closely allied may be briefly summarized as follows : Raps and similar noises varying in loudness on tables and other articles of furniture; the playing of musical instruments without contact of any kind; luminous appearances and materializations; and direct writing. Where the phenomena are systematic, and continue with but little intermission, we have a manifestation of what is commonly called a "poltergeist," who apparently haunts a particular person.

The physical phenomena thus recorded by psychical researchers, though differing in detail, conform more or less to a type, and for most purposes may be considered together. The first question to decide is whether any

${ }^{1}$ In some cases it is true that the medium must be entranced, but while in that state he is apparently quite passive. 
agent other than the medium is concerned. In this connection, it is noteworthy that when the phenomena occur while the medium is awake, he is frequently in a listless or idle condition, his attention wandering vaguely and not in any particular direction. In such cases it is observed that the manifestations are of a frivolous or nonsensical nature, or, where definite sentences are spelt out by means of raps, they are such as an intelligence on the same level as the medium's might be expected to give utterance to. In cases when the medium is entranced similar manifestations occur. On the other hand, the phenomena are sometimes due without doubt to agents different from the medium himself, and occasionally at a far higher intellectual level.

Quite apart from the explanation of the facts, then, we are probably justified in concluding that the phenomena fall into two classes. In some cases the medium is the only agent concerned; in others, agents different from the medium are also involved, although their operation depends in some way on the latter. Evidently a solution of the problem in cases where the medium alone is implicated, will almost certainly carry with it the solution of the further problem of the modus operandi employed by the discarnate agents.

In attempting to solve, as far as is possible, the very difficult problems raised at this point, it should be noted in the first place, that collective hallucination on the part of the observers of the movements of inanimate objects and the rappings therein, is not tenable, for the evidence is of exactly the same type as in the perception of normal occurrences, namely that of direct acquaintance. In the case of rappings, for example, the table 
(or whatever the object may be) can, if closely observed, be clearly perceived to be in the state of vibration to which such noises are normally due, although no cause of the vibration is perceivable. The same is the case with the playing of musical instruments. Hence such perceptions on the part of witnesses are no more collective hallucinations than are any other perceptions. The term, indeed, really means nothing in this connection, and is simply a cloak for ignorance.

Now our normal modes of interaction with those subjects of whom "inanimate" objects are the appearance, are evidently limited in number, being, in fact, manifested as to their results by the various types of sense-impression-sight, sound, etc. But there is no a priori reason for supposing that all possible modes of interaction are thus exhausted. If we first consider those cases when the medium is probably the only agent concerned, we may suppose that his interaction with his environment is taking an unusual form, this fact being manifested to the observers by listlessness, as regards attention to normal presentations, on the part of the medium if waking, which may pass into an actual trancecondition.

Assuming, then, that levitation of objects and sounds emitted by them are due to an abnormal type of interaction between the medium and the subjects of whom the physical objects are the appearance, the question arises as to whether we are here concerned with reciprocity of action or with community of action, that is, whether the type of interaction is perceptive or telepathic. If the former, we should expect it to be manifested to the medium not only by an abnormal correlation 


\section{Subconsciousness and Abnormal Phenomena}

of presentations which are themselves normal, but also by an abnormal kind of presentation not included in the list of ordinary sensory and motor presentations. There seems to be no record of mediums having been examined with a view to investigating this point, which is evidently a very important one. Apart from it, there is no direct method of determining the type of interaction involved. Possibly both types come into play to an extent governed in the case of each by the particular conditions involved.

When objects are moved, lifted, or rapped on, by normal methods (that is, by contact), the body of the agent evidently plays an important and necessary part in the process. This raises the point as to whether the body of the medium is particularly implicated when similar physical results are produced but by abnormal means. We may conclude that it is, from the general fact that after a sitting of this kind the medium exhibits considerable bodily fatigue. But the correlation between the normal and the abnormal phenomena would appear to be far closer than this, for in some recent experiments by $\mathrm{Dr}$ Crawford, it was found that in the levitation of objects, the weight of the medium increased by an amount equal to that of the object raised ${ }^{1}$, just as would have been the case had the object been raised by or-

${ }^{1}$ Cf. The Reality of Psychic Phenomena, by Dr W. J. Crawford, especially Chap. III. The experiments described show that there is some deviation from equality between the increase in the weight of the medium, and the decrease in the weight of the object levitated. From further experiments it also appears that the stress is probably distributed in some (at present) undetermined way among various objects, including the medium's body. But, in any case, the experiments show that the interaction involved is physical in type. 
dinary means. Hence, if this is confirmed by future experiments, it would appear that in occurrences of this kind, although certain important correlations of sensedata are broken down, others, no less important, remain unchanged. In any case, however, it is clear that the body of the medium must here play an especially important part, which is the only point that concerns us for the moment. For it provides an explanation of the fact that, even when the phenomena are apparently initiated by agents different from the medium, the presence of the latter's bodily organization is necessary to effect the required results.

We are probably justified, then, in regarding such phenomena as levitation and rapping as exhibiting action and reaction between the objects affected and the medium's body (whether the medium himself or another agent is the initiator of the process) strictly of the same type in essentials as ordinary contact action. If Dr Crawford's experimental results are confirmed, the physical law of equality of action and reaction holds here as elsewhere. Levitation of the medium while surrounding objects remain at rest is the correlative of the levitation of objects while the medium remains at rest; just as the action of a man in raising himself from the ground by pulling on a bar is the correlative of his action when he lifts a bar from the ground. The only difference between the two pairs of cases is that the latter is far commoner. The phenomena we have been considering certainly exhibit sense-data whose sequences and correlations are, in the present state of our knowledge, comparatively rare. That, however, is no reason for regarding them as being of a different order of facts 


\section{Subconsciousness and Abnormal Phenomena}

from commoner physical occurrences, especially, indeed, as there are, among the latter, sequences of essentially similar type. For, from a physical point of view, there is nothing more wonderful about levitation than about the repulsion or attraction of one magnetic pole by another, or of a pith ball by an electrified vulcanite rod. Action at a distance is, in fact, the characteristic of the controlling forces of the physical world through and through. In the case we are considering, what is called for, of course, is exact observation and quantitative experiment as a means of determining whether the phenomena may be described in laws of a physical type. In particular, in the case of levitation of the medium's body, indications of stress in surrounding objects should be sought for. If, for example, the medium were seated in a weighing-chair, it would be of considerable interest and importance to determine whether there were any change in the scale-reading of the instrument when levitation occurred.

But why, it may be asked, if these phenomena are physical in type, are they invariably connected with spiritualistic occurrences? Such a question is really quite irrelevant to the issue. To begin with, all phenomena, whether normal or abnormal, are the manifestation of the interaction of spiritual agents, and secondly, so far as communications from discarnate spirits are concerned, the method adopted more often than not consists in the use of the medium's body in a normal physico-physiological way-as, for example, in automatic speech, automatic writing by direct contact, etc. If the medium is capable of affecting objects by methods other than direct contact, it is only reasonable to sup- 
pose that the communicating agents might sometimes work through these as well as through more ordinary methods. This would imply that the medium ought himself to be able to initiate the unusual mode of interaction with surrounding objects when not controlled by other agents, and we saw, in fact, reason to believe that in many cases the medium is the only agent concerned.

There is, however, a more relevant question which might be raised at this point, namely why should the bodily organization of the medium be necessary at all for the production of movements in inanimate objects by discarnate agents? Why should not the latter act on the objects directly? It might be replied that this fact requires no more and no less explanation than the fact that we ourselves invariably act on objects other than our body in association with the latter, and not independently. It is conceivable that, quite apart from the functions and purposes of the body in relation to its dominant monad which we previously investigated ${ }^{1}$, no purposeful interaction is possible between a dominant monad at a high level and those monads of which "inanimate" objects are the appearance, except in so far as the former is associated with a body. Put in its most general form the question is: "Why can purposeful interaction between a monad at a high level of experience and others at an indefinitely lower level only take place when the latter is associated with an organized hierarchy of monads at various levels?" It is impossible to discuss the question fully here. An analogy must suffice to indicate an answer which appears likely. If one person wishes another to respond in a

${ }^{1}$ In the previous essay. 


\section{Subconsciousness and Abnormal Phenomena}

certain way to a command or desire of his, the second person being on an entirely different plane of life (due, for example, to differences of race, education, or social position), the quickest, and perhaps the only, way of producing the required relevant response, is through the instrumentality of one or more intermediaries. Only thus can one level of experience with its associated complexes of images and concepts be satisfactorily linked up with another and entirely different one ${ }^{1}$.

In some such way as the foregoing may we interpret the physical phenomena of levitation, rapping, and direct writing. They manifest an employment of the medium and his body in ways strictly analogous to the more usual methods adopted, namely contact action of various kinds. The analogy is of the same type as that between the gravitational attraction and the contact action of any two physical bodies on one another. There remain, however, such phenomena as luminous appearances and materializations to be accounted for. But until these phenomena have been more closely observed, and the conditions under which they occur more fully investigated, it is impossible to attempt any satisfactory explanation of them. All that can be said at present is that the data available clearly show that the processes which they manifest are dependent on the body of the

${ }^{1}$ If such an explanation of this function of the body were true, it would follow that disembodied subjects could perceive and commune with each other directly, association with a body being unnecessary, although they might be unable to interact purposively with subjects of which inanimate objects are the appearance, and hence unable to perceive the latter with any distinctness. This point has an important bearing on the topic discussed at the end of Section VI of the previous essay. 
medium to at least as great an extent as are the other phenomena we have been discussing. They have, in fact, been invariably found to occur with mediums whose presence is also accompanied at times by levitation and other allied physical phenomena.

\section{Conclusion}

Looking back at the results we have been led to in connection with the various important points raised in the course of the foregoing investigation, it is not unfair to say that, broadly speaking, the strange facts which, at the present time, are receiving a continually increasing amount of attention from all those who are concerned solely for the advancement of knowledge in no matter what sphere, are in every case susceptible of interpretation and explanation (so far as such are capable of expression in words at all) by means of the general metaphysical hypothesis we have adopted as to the structure of reality. Regarding the latter as a plurality of individual entities constituting a universe in virtue of the immanence in them of a single entity, it might be supposed, in so far as these entities are spiritual agents, that the phenomena we have been considering are rather of a type to be expected than to be regarded as bizarre and utterly dissociated from ordinary life. For throughout our examination one point has continually been in evidence, and that is the essential continuity between the normal and the abnormal, regarded from the standpoint of experience. So far as the individual is concerned, we pass continuously from the suspension of certain usual forms of activity 
which is manifested in such everyday occurrences as dreams (both waking and sleeping), to the phenomena of the hypnotic trance and of dissociated personality. The transition to the latter is effected largely by means of that group of latent tendencies manifested by the subliminal images which make up the subliminal self, the latter constituting part of the ordinary empirical self. Dissociated personality is therefore simply due to a single subject acting at different times according to conceptions of himself which differ widely. It is continuous with such differences as are manifested in the general mode of behaviour of the same man at home (say) and in business.

On the other hand, so far as the Many and their interaction are concerned, we pass continuously from normal perception, through the mediation of impressions which are normally ultraliminal, to such abnormalities as clairvoyance and clairaudience of distant or hidden objects, and the perception of apparitions. But probably the ground of the latter is frequently not perceptive but telepathic. The community of action between different individuals which the latter manifests is not prima facie continuous with normal processes. But it is extremely likely that among these processes are many which are telepathic in nature though not recognized as such. We have at least one striking example in the common impulse of the mob.

Granted, in accordance with our ontology, the existence of discarnate agents, the facts of mediumship may be largely explained by regarding the action of these agents on the medium as for the most part hypnotic. But in some cases it is necessary to suppose that 
the operator controls the body of the medium, not indirectly by hypnotizing the latter, but directly in a manner similar to that in which it is normally controlled by the medium himself. As to the way in which this is effected, we can at present only conjecture within very wide limits, without being able to come to any definite conclusion. But seeing that our direct knowledge must, at most, be limited to one side of the process, it would be very rash to assert dogmatically that we are here faced with a complete breach of continuity with normal processes.

Finally, we may regard levitation and similar phenomena, though unusual, as exhibiting actions and reactions between the body of the medium (considered as a material object) and other material objects, which are strictly physical in type. They are no more remarkable than other well-known physical phenomena manifesting action at a distance, with which they are comparable ${ }^{1}$. As for their connection with spiritualism, it arises simply from the fact that the controlling agent may use the body of the medium in any way in which it is capable of acting. To every type of mediumistic action at a distance, there corresponds a type of mediumistic action by contact with the objects affected. The only problem here is, therefore, strictly an empirical one, to be investigated by the methods of physical science. In the first place, exact and quantitative observations are necessary with regard to the accompanying circumstances of the phenomena ; secondly, a determination of the charac-

1 It may also be noted that visual perception is, from a physical point of view, an interaction between the body of the percipient and other objects at a distance from it. 
teristics of those persons (or of their bodily organizations) capable of producing the phenomena, which are lacking or suppressed in most people; and thirdly, if the results obtained in the two previous cases appear to support the possibility, to determine whether and how this physical power may be developed and exercised by any individual.

It may be concluded, then, that it is impossible to overestimate the profound importance of the phenomena commonly grouped under the heading of "spiritualistic." This being so, it is the harder to understand the jibes of those who will not trouble to investigate the facts for themselves first hand, under conditions set in accordance with the most rigorous principles of scientific stringency. Fortunately the company of impartial psychical researchers is a rapidly increasing one. As a result of their labours we may confidently hope that the light already shed on the nature of the universe by the consideration of these remarkable phenomena, will spread to the dark corners where ignorance lurks most obstinately, and will increase in intensity a hundredfold. 


\section{CONCLUSION}

The present work may perhaps be most fittingly concluded by a brief review of the results so far achieved. The unit with which we have been dealing throughout, is the individual experience. Two facts of supreme importance stand out in connection with the twin factors, subject and object, distinguished in the latter, namely the indivisibility of each in its actuality, and the inseparability of either from the other as regards its being. In respect of the last, the existential emphasis is on the subject ; the being of the object, though dependent on the existence of the subject, yet coming under a type different from that of the latter.

A clue to the interpretation of these facts is furnished by the hypothesis that reality is made up of interacting subjects, the object of experience for each subject being the manifestation to him of the form to which his activity is determined by his interaction with others. The ground of this interaction must lie in a single, universally immanent, concrete entity, whereby there subsists between each subject and every other subject, a "sympathetic rapport." In the case of certain groups of subjects, there subsists a peculiar relation between one who dominates the group and the other members composing it. It is to groups of this kind that we give the name of "organisms."

The pluralistic universe (and this paradoxical phrase fitly expresses our view of reality) as thus conceived is 
a system transcending space and time, for the latter, far from being noumenal, are but abstractions from the phenomenal. Thus in the final synthesis to which the system is the corresponding concrete reality, the principles of permanence and change, the static and the dynamic, are fully reconciled. We have, not a succession of elements, but a system ("society" is perhaps a better word) of single, indivisible interacting subjects ; not an eternal nunc stans, nor a temporal omnia mutabilia, but a reality in which both are transcended.

The nature of a universe of this kind can evidently not be expressed discursively, nor even approximately described in any detail. How the temporal and spatial are transcended, and permanence and change reconciled, we cannot put into words, for words refer almost exclusively to objects which are spatial and temporal. But we can realize the nature of this transcendence and reconciliation, for it is in such active individuals as ourselves that it exists. The dynamic, changeful, element is present in so far as the individual is an active individual; the static, permanent, element, in so far as it is a single indivisible entity who acts. Thus in the self we have a being who is neither a mere permanence, nor a mere flux of elements, but one whose nature transcends and reconciles both.

From this point of view such conceptions as freedom and immortality take on a new aspect. We have considered the former from the position of the individual experience, and more or less from within that experience, as it were. But what of the problem in its more particularly noumenal implications? Here the spatial and temporal have no place as such, and we are 
faced with the question as to what in reality is expressed by the concept of freedom. It is not for us to discuss the point here, for it takes us beyond our present limits ; but it may be said that perhaps the answer is to be found in the consideration that the individual is free in so far as he is individual, i.e., as his nature is absolutely unique, and not capable of being deduced from the nature of anything else.

The concept of immortality, again, must take new shape, ridding itself of its former temporal reference. The question is not whether the existence of the subject is without beginning and without end, for such phrases have here no significant application. We can only say of any individual: "Does he exist or not?" and if so, there is the further question as to what is the absolute ground of his existence; is it in any respects other than himself, and if so in what respects?

In other words, such time-honoured problems as freedom, immortality, creation, and the existence of God, would seem on analysis to strike down to some common root. And this fact sufficiently indicates the direction in which further enquiry must be prosecuted, when we attempt to advance from the position we have so far reached. For the final answer to these questions must somehow lie in the determination of the nature of that concrete universal entity, in virtue of whose immanence the plurality of selves is no mere plurality, but a universe. If the nature of this ground of the world can be elucidated in its essentials, it will be possible to say whether the name "God," with all that that name implies, can fitly be given to it; and by the further examination of the relation of God to the 
plurality of individuals, it will be possible to obtain a precisely significant statement of the problems associated with such terms as creation, freedom, and immortality, and perhaps to reach as adequate as possible a solution of those problems. The ain of a philosophy which may claim to interpret and explain the world in a fashion that shall have full and satisfactory meaning for such individuals as ourselves, must therefore supplement its examination of the Many by the examination of the One, proceeding finally to an intuitive conception of that reality in which both co-exist. 


\section{INDEX}

Abnormal phenomena, 26I ff.

Absolute, the, 72

Acquaintance, 68, 70, 90, I 39, 239

Action at a distance, 38, 322, 327; and telepathy, 302

Activity, I7, 3I ff., 48, 6I f., 97 f., I 37 ff., I 42 f., I 53 f., I 94,223 f., 234 f., 249 ff., 254 ff., 260, 262 ff., 266 ff., 289, 299, 30I, 329 f.

Analysis, validity of, $173 \mathrm{ff}$.

Apparitions, see Phantasms

Appearance, 69 f., 80, 99 ff., I Io, 197

Apperception, 253, 255

Appreciation, $73 \mathrm{f}$.

Association, 223

Atom, the, 79

Attention, 138, 141, 143, I54, I60 f., I 94, 209, 223 f., 248, 259, 262, 265, $284,288 \mathrm{f}$.

Attribute, $39 \mathrm{ff}$., $47 \mathrm{f}$.

Automatic writing, 275, $304 \mathrm{ff}$.

Barrett, Sir W. F., 273 n.

Beauchamp, Sally, 282

Birth, 316

Body, the, xvii, xix, 7 I ff., 78 f., I 8 I ff., I $92 \mathrm{ff}$., 200, 30I, 3I 5, $323 \mathrm{f}$.

Bosanquet, Bernard, $66 \mathrm{ff}$., 80 , $184 \mathrm{n}$.

Bradley, F. H., 32 n., 50

Categories, of experience, xvi, I6, $39 \mathrm{ff}$.

Causality, $30 \mathrm{ff}$., 6o, I 17 f., 120, 20I f.

Change, 39 ff., 330

Clairvoyance and clairaudience, 205 , 283 ff., 308

Communicator, $306 \mathrm{ff}$.

Consciousness, 7 I ff., 80 f. ; self-, 209 f., 233, 242 ; sub-, see Sub-consciousness ; field of, $247 \mathrm{ff}$., 265 ; focus of, $247 \mathrm{ff}$., 259 ; threshold of, $248 \mathrm{ff}$.

Continuity, $22 \mathrm{ff}$., $6 \mathrm{I}$

Control, 277, $306 \mathrm{ff}$.

Crawford, W. J., 320, $32 \mathrm{I}$

Creation, 79, $33 \mathrm{I}$

Crystal-gazing, 284
Data, of sense, see Sense-data

Death, 209, 217, 219, 230 f., 236, 313; life after, xviii, $x x, 162,180$, $236 \mathrm{f}$., $292 \mathrm{ff}$.

Descartes, xix, 2, 92, I85, 237

Determinism, xvii f., 35 ff., I I $5 \mathrm{ff}$.

Disease, $269 \mathrm{ff}$.

Dreams, $262 \mathrm{ff}$.

Dualism, xix, 7 I, I84 ff.

Ego-centric predicament, I $88 \mathrm{ff}$.

End, $5 \mathrm{I} \mathrm{ff}$., I $46 \mathrm{f}$.

Energy, conservation of, $186 \mathrm{f}$.

Environment, 75, 204

Existence, 42, 46, $68 \mathrm{ff}$., 80, I63

Experience, unity of, xix, $18,23 \mathrm{ff}$., 49 f., 6r, 7 I, 92, I 36 , I 73 ff., 179 f., I99, 329; continuity of, $22 \mathrm{ff}$., $6 \mathrm{I}$, I7 5 f. ; nature of, $92 \mathrm{ff}$, I 163, I 87

Explanation, 7, I 3 ff., 64, 202

Externality, $67 \mathrm{ff}$., 80

Familiarity, $222 \mathrm{f}$.

Fechner, 84, I 32, I 57

Feeling, I 37, I 39, I 4 I f., I 53, I 94

Forgetting, 228

Free will, xvii, I I6, I 45 ff., I 54, 330 f.

God, 33I

Gurney, Edmund, 266, 275

Haeckel, Ernst, 207 n.

Hallucination, 272 ; collective, $3 \mathrm{I} \delta \mathrm{f}$.

Haunting, $294 \mathrm{f}$.

Hegel, i 6

Heraclitus, 39

Hume, 22 I n.

Hypnotism, 265 ff., 3 I I ff., 3 I 5 f.

Hypothesis, $12 \mathrm{ff}$.

Idealism, xvii

Illusion, $32 \mathrm{f}$.

Imagination, $x x, 220$ ff., 24 I f., 245 f., $252 \mathrm{ff}$.

Immanence, 2 I 2 ff., 240, 3 I 3 ff.

Immortality, xvii f., I62 ff., $330 \mathrm{f}$.

Immunity, from pain, etc., $270 \mathrm{f}$. 
Impressions, of sense, 22 I ff., 241 f., $245 \mathrm{f}$., $247 \mathrm{ff}$.

Individual, $29 \mathrm{f}$., $33 \mathrm{I}$

Insanity, 276, 279

Intellection, 233

Intensity, of sense-data, see Sensedata

Interaction, 79, 185 f., 203 f., 250, 285 ff., 29I, 3 I 9 ff., 329

James, William, 194 n.

Knowledge, xiv, 2 I, 90

Laplace, I 24 f., I 40 , I 50

Laws, causal, $3 \mathrm{I}$; evolution of natural, $75 \mathrm{ff}$., $8 \mathrm{I}$ f.

Leibniz, 66, 78, 200, 2 16 n.

Levitation, 317 ff., 327

Locke, 206, 226

Lotze, 2 I $3 \mathrm{n}$.

Lourdes, miracles of, 270

Materialism, 9

Materialization, 317

Matter, xix, 8, 9, I I, 53, 72 f., 76 f., $92,126 \mathrm{f}$.

McDougall, W., 282

McTaggart, J. M. E., xiii n., 22 I $\mathrm{n}$.

Meaning, 73 f., 2 ro

Means, 5 I

Mechanism, I 22 f., 149

Medium, psychic, 282, $304 \mathrm{ff}$., $326 \mathrm{f}$.

Memory, xx, $220 \mathrm{ff}$.

Method, scientific, I, 4 ff., I6, 29, 54 ff., 62 f. ; genetic, 12, 16, 59

Mind, xvii, xix, 8 f., 7 I ff., 92, I06 f., I 26 f., I 37 ff., I 8 I ff., I 94 f., 200

Minkowski, 42, 45

Monads, 75, 78 ff., 82, 200 ff., 323 f.

Newton, 135

Number, I 30

Object, of experience, 6, $16 \mathrm{f}$., 28 , 52,58 f., 68 ff., 92 ff., 102, 108 ff., 166 ff., 169 ff., 173 ff., 250, 260, 329

Occam's razor, 16, 104

Organism, 72 f., 78, 207 ff., 240, 329
Parallelism, psycho-physical, 122, 1 24, 1 49, 187, 238

Part, I73 ff.

Perception, abnormal, 205, 2 19, 257, 261, 264, 283 ff.

Permanence, $39 \mathrm{ff}$., 330

Perry, R. B., 7 n., 29 n., 32 n., 93 n., 94 n., $188 \mathrm{n}$.

Personality, xx, 232 ff., 305, 310

Phantasms, $288 \mathrm{ff}$.

Philosophy, nature of, xiii f.

Physics, objects of, 79, I03 f., I I I, I 33 ff., 190 f., 238

Planchette, 275

Pluralism, vii, xv f., 3, $8 \mathrm{ff}$., $53,58 \mathrm{ff}$., 63 f., 65 ff., 75 ff., I04, I 12 f., I $98 \mathrm{ff}$., 206, 239, $329 \mathrm{ff}$.

Poltergeist, 317

Presentation, relation of, $92 \mathrm{ff}$., $108 \mathrm{f}$., 194

Prince, Morton, 282

Pringle-Pattison, A. S., 66, 69, 75 ff., 8 I f.

Psychical research, xx, $244 \mathrm{ff}$., 328

Purpose, $51 \mathrm{ff}$., $146 \mathrm{f}$.

Quality, 48 ff., I28, I 32, 136 f., I60 f. Quantity, I27f., I 30 ff., I 5 Iff., I 55 ff.

Rapport, 2 I $3 \mathrm{f}$.

Raps, psychic, $317 \mathrm{ff}$.

Realism, New, vii f., xvi, I, $4,16 \mathrm{ff}$., $33,85,93$ f., 98 ff., I Io f., $188 \mathrm{f}$.

Realization, I 3 f., I9, Ior, I 39, 202

Recognition, $222 \mathrm{f}$.

Reflexes, 72, 8I, $207 \mathrm{f}$.

Relation, $26 \mathrm{f}$., $48 \mathrm{ff}$.

Relativity, principle of, $45 \mathrm{f}$.

Russell, Bertrand, vii f., xiii n., xvi, I n., 4 n., 17, 18, 24, 26 ff., 35, 56, 85 n., 90,95 ff., 99 f., 102 ff., 107 ff., I 9 ff., I 28 f., I 30 n., I 48 f., I 52 , 175 n., $191 \mathrm{n}$.

Science, concepts of physical, $5 \mathrm{f}$., I I I, $133 \mathrm{ff}$., I9I

Self, as subject, $8 \mathrm{ff}$., I $3 \mathrm{f}$., 43,62 , $67,146,258 \mathrm{f}$; ; existence of, $18 \mathrm{ff}$., $44 \mathrm{f} ., 58,63,163$; empirical, I9, I 94, 232, 259 f., 278 ff., 326 ; content of, 234 ; subliminal, see Subliminal self 
Self-consciousness, see Consciousness

Sensation, 84 ff., 21 I ff.

Sense-data, intensity of, 49, 132, $155 \mathrm{ff}$; differences in, $86 \mathrm{ff}$., IO5 f.; series of, $24 \mathrm{ff}$; ; being of, $95 \mathrm{ff}$., IOI f., $195 \mathrm{ff}$., 225 , 239; general characteristics of, 5 f., 10,59

Sensibilia, 96, 100, 102, $108 \mathrm{f}$.

Sensibility, general, 193, 199

Sleep, 166, 17 I, 262 ff., 265 f., 267, 290, $29 \mathrm{I}$ n.

Solipsism, 5, 2I f., I 70, 197

Space, $x v i, 42 \mathrm{ff}$., $33^{\circ}$

Spaulding, E. G., $174 \mathrm{n}$.

Spencer, Herbert, 9, 197

Stigmata, 271

Stimulus, 84 ff., I 57, 210

Stout, G. F., 85 n., 88 n.

Subconsciousness, 85, 234, $244 \mathrm{ff}$.

Subject, of experience, vii f., 8, 9, $14,17,19,43,56$ ff., 68 ff., 92 ff., IOI f., $108 \mathrm{ff}$., I $37 \mathrm{ff}$., $144 \mathrm{f}$., I $66 \mathrm{ff}$., 169 ff., $173,194,223$ ff., 249 ff., 256 , 258 f., $277,285,329 \mathrm{ff}$.

Subliminal images, 246, 252 ff., 326 ; self, 256 ff., 263,326

Substance, $39 \mathrm{ff}, 47 \mathrm{f}$.

Suggestion, $265 \mathrm{ff}$.
Teleology, 5 I ff., I22 f., 146 f., I49, 207, 210

Telepathy, 283, 284, 295, $297 \mathrm{ff}$., 311,326

Tendencies, subjective, 255 ff., 260 , $266 \mathrm{ff}$.

Theism, 10, $203 \mathrm{n}$.

Time, xvi, $36,40 \mathrm{ff} ., 43 \mathrm{ff} ., \quad 164 \mathrm{ff}$, $178 \mathrm{f}$., 330 ; appreciation of, 273

Trance, 265 ff., 283 f., 305 ff., 3 II f.

Unity, of experience, see Experience; of reality, xxi, $79 \mathrm{f.}, 82 \mathrm{f}$., 203 f., 250, 285, 286, 299 ff., 325, $329 \mathrm{ff}$.

Ultraliminalimpressions, $245,247 \mathrm{ff}$., 285 f., 326

Volition, 21 I ff., 219 f., 288, 289 f.

Ward, James, 9, Ion., 20 n., 65, 72 n., 75, 85 n., 92 n., I6on., 203 n., 207 n., 209 n., 212, 213, 215 n., 222 n., 223 n., 228 n., 229 n., 232 n., 234, 245 n., 246 n., 278 n.

Weber, 84

Weber-Fechner Law, $84 \mathrm{ff}$.

Whitehead, A. N., I n., IO2, I30 n.

Whole, $173 \mathrm{ff}$.

Wundt, 253 





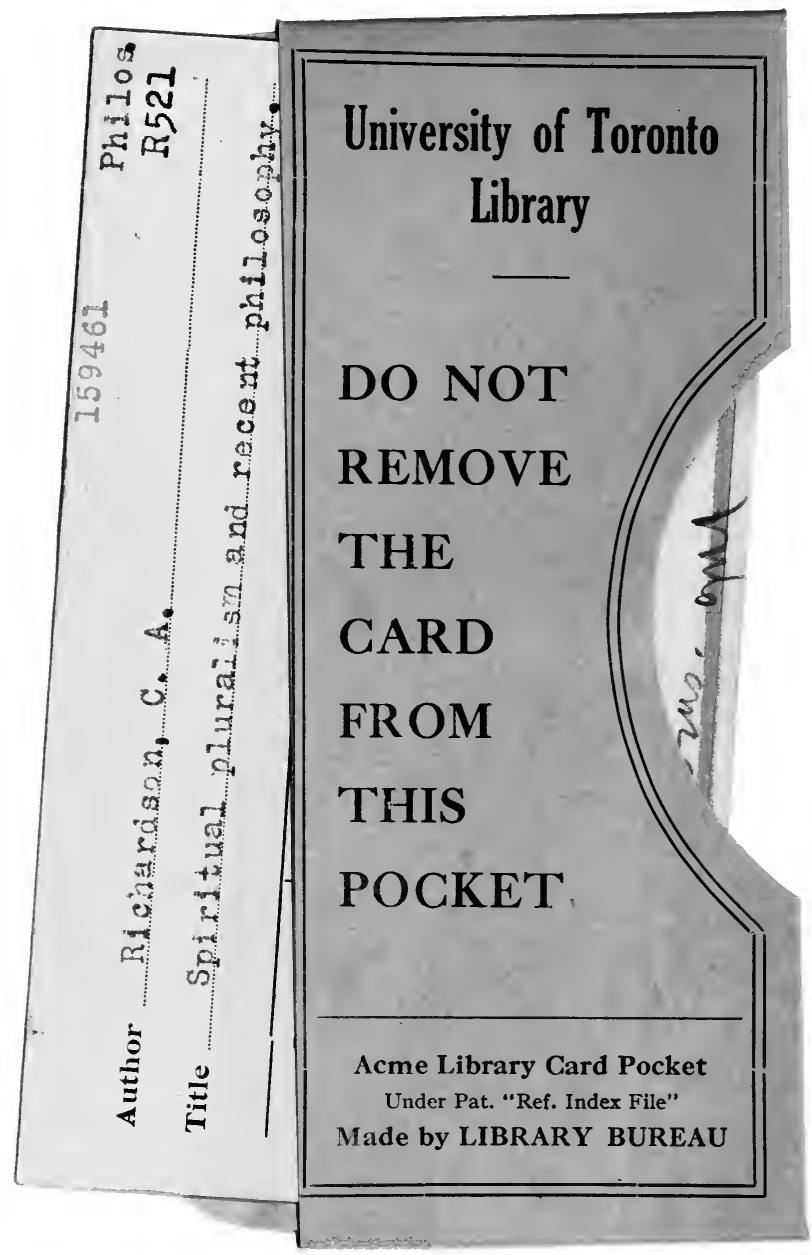


